\title{
Empirical Studies Related to Open Questions Regarding Geomagnetic Storms
}

\author{
Bruce Patrick Tepke \\ WVU, btepke@mix.wvu.edu
}

Follow this and additional works at: https://researchrepository.wvu.edu/etd

Part of the Plasma and Beam Physics Commons

\section{Recommended Citation}

Tepke, Bruce Patrick, "Empirical Studies Related to Open Questions Regarding Geomagnetic Storms" (2019). Graduate Theses, Dissertations, and Problem Reports. 3834.

https://researchrepository.wvu.edu/etd/3834

This Dissertation is protected by copyright and/or related rights. It has been brought to you by the The Research Repository @ WVU with permission from the rights-holder(s). You are free to use this Dissertation in any way that is permitted by the copyright and related rights legislation that applies to your use. For other uses you must obtain permission from the rights-holder(s) directly, unless additional rights are indicated by a Creative Commons license in the record and/ or on the work itself. This Dissertation has been accepted for inclusion in WVU Graduate Theses, Dissertations, and Problem Reports collection by an authorized administrator of The Research Repository @ WVU.

For more information, please contact researchrepository@mail.wvu.edu. 


\title{
Empirical Studies Related to \\ Open Questions Regarding \\ Geomagnetic Storms
}

\author{
Bruce Tepke \\ Dissertation submitted to the Eberly College of Arts and Sciences \\ at West Virginia University \\ In partial fulfillment of the requirements for the degree of \\ Doctor of Philosophy \\ in \\ Physics
}

Mark E. Koepke, Ph.D., Chair

Earl E. Scime, Ph.D.

Dimitris Vassiliadis, Ph.D.

Weichao Tu, Ph.D.

Wade Huebsch, Ph.D.

Department of Physics and Astronomy

Morgantown, West Virginia

2019

Keywords: magnetosphere, geomagnetic storms, substorms, empirical modeling

(C)2019 Bruce Tepke 


\title{
Abstract \\ Empirical Studies Related to Open Questions Regarding Geomagnetic Storms
}

\author{
Bruce Tepke
}

Earth's magnetosphere is subject to disturbances, as evidenced by variations of the geomagnetic field in space and on the ground. It is generally understood that most such disturbances are controlled by variations in the solar wind, with interplanetary magnetic field orientations directed southward opposite to Earth's dipole magnetic axis being most conducive to energy transfers into the magnetosphere, thus resulting in more disturbed intervals. However, the exact functional form for solar wind driving of the magnetosphere has been widely studied, with proposed functional forms varying from the simple half-wave electric rectifier to expressions with a much more complicated dependence upon solar wind parameters. We provide evidence that past empirical results favoring more complicated expressions can statistically emerge from simpler expressions when solar wind parameters are time averaged and that expressions found in past empirical studies can be at least partly explained by the use of time-averaged solar wind parameters having hourly timescales, leading to the pitfall of assigning profound physical meaning to a statistical accident. Suggestions are offered to avoid this pitfall in future investigations.

The strongest and most expansive disturbances in the magnetospheric system are magnetic storms. The signature of a geomagnetic storm is the reduction in the strength of Earth's magnetic field at low latitudes. The conventional explanation for this storm-time geomagnetic depression is a ring shaped current system in the near Earth magnetosphere. In recent years, this conventional view has been called into question by researchers who argue that much of the depression is caused by currents in the more distant region called the magnetotail. Many researchers in the field continue to accept the conventional view. The relative contributions of the current systems are still debated.

We construct impulse response functions (IRFs) for storm-time depression to shed light on this controversy. We show that the reduced driving of the geomagnetic index SYM/H (used to measure storm magnitude) during intervals of low density solar wind is due to energy diversion to the ionosphere via burstier events called substorms. As substorm energy is derived from the magnetotail, this reduced driving of storms when substorms are enhanced implies that tail currents are significant to storm-time indices. We also note that the storm-time magnetic depression IRF has a second development several (2-7) hours after the solar wind transfers energy to the magnetosphere, which is more prominent when energy is diverted from the tail to the ionosphere. The IRF of that part of storm-time magnetic depression due (theoretically) to tail currents, as inferred from Auroral Boundary Index (ABI), is shown qualitatively similar to the IRF for SYM/H prior to the second development. We are able to show, by adding functions of AL as an additional IRF driving variable, that this second development is likely due to substorm activity. We interpret this as being consistent with the hypothesis of ionospheric $\mathrm{O}+$ ions enhancing the ring current with a time delay of approximately 2-5 hours. Evaluation of IRFs for sector SMR indices (which resolve stormtime magnetic depression into zones by magnetic local time sectors) reveals a more complicated picture, with evidence for gradual symmetrization of ring current. We model an ideal IRF using our hypothesis and, by comparison to data generated IRFs, show that it presents a plausible model.

As Maltsev's derivation [Maltsev (1996); Maltsev et al. (1996)] of tail current contributions to storm-time magnetic depression depends upon the extent of the equatorward auroral oval, the problem that K-family indices are widely regarded as auroral latitude proxies, rather than stormtime magnetic depression indices, presents itself. We show that the relation of Kp to ABI stems from 
the quasi-logarithmic scaling of Kp, and that storm-time indices, particularly Dcx when corrected for solar wind ram pressure effects, are also a good proxy for ABI when scaled logarithmically.

We use ionospheric field aligned current (FAC) maps, provided by APL's AMPERE project, to generate statistically averaged FAC maps via the machine learning technique of k-means clustering. The region 2 (R2) currents are identified for each cluster and used as a proxy for the equatorward edge of the auroral oval. The magnetic flux in the auroral oval is then used to calculate a predicted tail current contribution to storm-time magnetic depression according to Maltsev's theory. Remarkable agreement between the predicted and observed median pressure corrected Dcx is found, suggesting that tail contributions are a majority contribution to storm-time magnetic depression. 


\section{Acknowledgements}

When I came to WVU, in the summer of 2009, Earl Scime provided me with the opportunity to get my feet wet in plasma research as a temporary member of his group. Thanks to Dr. Scime for making it a positive experience. I also want to mention that a number of his group members were especially generous in sharing their time and knowledge during this time, including Michael Lindon, Saikat Chakraborty-Thakur, Stephanie Sears, and Saeid Houshmandyar.

When I found the research opportunity that eventually culminated in this dissertation, it was with Dr. Dimitris Vassiliadis. Dr. Vassiliadis very patiently introduced the concepts of space physics and empirical modeling, and was always generous with his time and advice during while he served as my advisor. I am gratefull to have had the opportunity to have learned so much from him.

Thanks to Dr. Mark Koepke, who took over as my advisor when Dimitris left WVU. Dr. Koepke has been very generous with his time and I can see first hand how energetic and creative he can be when it comes to helping his students.

Thanks to Dr. Dimitris Vassiliadis, Dr. Earl Scime, Dr. Wade Huebsch, Dr. Paul Cassak, and

Dr. Weichao Tu for serving on my graduate committees. Special thanks to Dr. Weichao Tu, who was willing to quickly become a member of my defense committee on such short notice.

Thanks to Dr. Amy Keesee, who provided funding for my work from her grant during my time as a full-time student.

Thanks to all those who were generous to Xingwei and myself after the fire. Recovery from such an event is easier when aided during the darkest hours. I will pay it forward.

My sincere thanks to Phil Tucker and Greg Puskar, who always made an effort to lend a helping hand. They are missed.

Thanks Sherry Puskar and Viola Bryant for their efficient and friendly help in the office.

Thanks to those teachers who were influential in nurturing my scientific interests during my formative years, especially Mr. McCarthy, Mr. Yoworski, Mrs. Jeanine McCutcheon, and Mr. Richard Gittins.

I want to express my affection and gratitude to Wayne and Amelia Tepke for being excellent parents and supporting my thirst for knowledge while growing up. 
I dedicate this work to my family. I am so proud of my son, Victor Wu Tepke. Thanks to him for being such a wonderful child. Special thanks to my wife, Xingwei Wu Tepke. She did much to encouraged me while I spent my evenings focusing my attention on space physics instead of her. I am fortunate to have such a wonderful family and look forward to many more years with them. 


\section{Table of Contents}

$\begin{array}{ll}\text { Abstract } & \text { ii }\end{array}$

$\begin{array}{ll}\text { Acknowledgements } & \text { iv }\end{array}$

List of Tables $\quad$ viii

List of Figures $\quad$ xii

I Introduction $\quad 1$

A. The Magnetosphere . . . . . . . . . . . . . . . . . 1

1. The Solar Wind . . . . . . . . . . . . . . . . . . . . . . 1

2. The Magnetopause and Cusp . . . . . . . . . . . . . . . . 2

3. The Magnetotail . . . . . . . . . . . . . . . . . . . . . 2

4. The Inner Magnetosphere $\ldots \ldots \ldots \ldots$

5. The Ionosphere . . . . . . . . . . . . . . . . . . . . 4

B. Quantifying Magnetospheric Behavior . . . . . . . . . . . . . . . 4

1. Storm Time Indices: $D_{S T}, \mathrm{SYM} / \mathrm{H}, \mathrm{Dcx}, E_{S T}$, and SMR . . . . . . . 5

2. Auroral Electrojet Indices: AL, AU, and AE . . . . . . . . . . . . . 6

3. Global Variation based Indices: Kp, ap, am, am-sector and $\alpha 15 \ldots \ldots$

4. Auroral Boundary Index and the MT Index $\ldots \ldots \ldots \ldots$

C. Modeling Techniques . . . . . . . . . . . . . . . . . . . 8

1. Physics-Based Modeling . . . . . . . . . . . . . . . . . . . 8

2. Empirical Modeling . . . . . . . . . . . . . . . . . . . . . . 9

D. Geomagnetic Storms and Substorms . . . . . . . . . . . . . . . . 12 
1. Geomagnetic Storms . . . . . . . . . . . . . . . . . 12

2. Geomagnetic Substorms . . . . . . . . . . . . . . 15

3. Controversies Concerning the Storm-Substorm Relationship . . . . . . . . 15

II Rectified Solar Wind Electric Field: Time Scale Effects 17

A. Solar Wind Statistics and Coupling Functions utilizing Hourly Averages in Solar

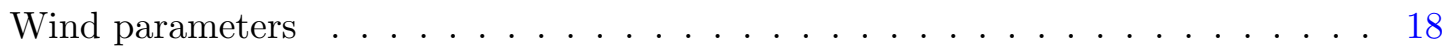

B. How Solar Wind Coupling Functions Using Hourly Solar Wind Variability Compare with Other Widely Used Functions . . . . . . . . . . . . . . 20

C. Burton Type Differential Equation Models of Geomagnetic Storms . . . . . . . . . 21

1. Optimizing the Burton Model and its Injection Function . . . . . . . . . . . 21

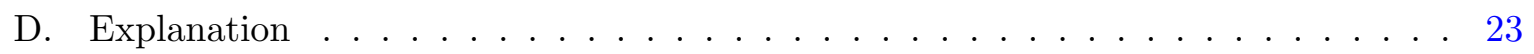

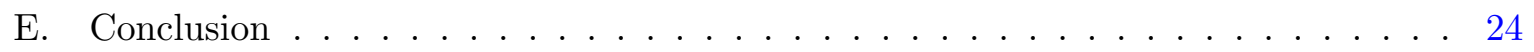

III Impulse Response and The Storm-Substorm Relationship 25

A. Impulse Response Function Methodology . . . . . . . . . . . . . . . . . 26

B. Evaluation of The Solar Wind Dependence of $D_{S T} \ldots \ldots \ldots$. . . . . . . . . 27

C. A Comparison of Sym/H and AL Impulse Response under High and Low SW Density Conditions . . . . . . . . . . . . . . . . 28

D. Substorm Activity, Tail Currents and Sym/H Impulse Response . . . . . . . . . . 29

E. Sector SMR Index Impulse Responses . . . . . . . . . . . . . . . . . . . . . 31

F. F. Discussion and Conclusions . . . . . . . . . . . . . . . . 32

IV Evidence of Tail Current Contributions to Magnetic Storms 35

A. Auroral Boundary Index . . . . . . . . . . . . . . . . . 36

1. K-Family indices versus ABI . . . . . . . . . . . . 37

2. Storm-Time Magnetic Depression Indices versus ABI . . . . . . . . . 37

3. Pressure Corrected Storm-Time Magnetic Depression versus ABI . . . . . . 38

4. Sector SMR Indices versus ABI . . . . . . . . . . . . . . 38

5. K-family Indices versus Storm-time magnetic depression Indices as Proxies for Auroral Expansion . . . . . . . . . . . . . . . . . 38 


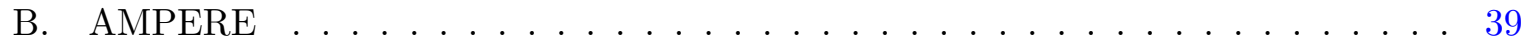

1. Locating FAC Currents Using AMPERE and K-Means Clustering . . . . . . . 39

2. Relating Clusters to Geomagnetic Activity . . . . . . . . . . . . . . . . . 40

C. Summary and Conclusions . . . . . . . . . . . . . . . . . 41

$\begin{array}{ll}\text { References } & 43\end{array}$

$\begin{array}{lc}\text { Tables } & 53\end{array}$

$\begin{array}{lc}\text { Figures } & 62\end{array}$ 


\section{List of Tables}

1.1 A listing of magnetic observatory stations used to compute $D_{S T}$. From the World geomagnetic Data Center at Kyoto (http://wdc.kugi.kyoto-u.ac.jp). . . . . . . . . . 54

1.2 A listing of magnetic observatory stations used to compute SYM/H. From the World geomagnetic Data Center at Kyoto (http://wdc.kugi.kyoto-u.ac.jp). . . . . . . . . . 54

1.3 A listing of magnetic observatory stations used to compute Dcx index. From the

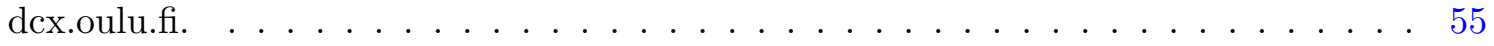

1.2 A listing of magnetic observatory stations used to compute SYM/H. From the World geomagnetic Data Center at Kyoto (http://wdc.kugi.kyoto-u.ac.jp). . . . . . . . . 55

2.1 Some commonly encountered solar wind coupling functions are shown. In the above, $\theta_{C}=\arctan \left(B_{Y} / B_{Z}\right)$ is IMF clock angle, $N$ is $\mathrm{SW}$ proton density, $v$ is the (antisunward) SW speed, $B_{S}$ is the rectified IMF componant directed in the -Z GSM direction, and $B_{T}$ is the IMF in the plane perpendicular to the Earth-Sun line. . . . 56 
2.2 The mutual cross-correlations of solar wind coupling functions computed using all available data from the 1973 - 2015 Omni databases. The functions $<v_{\min } B_{S, \min }>$ and $\sqrt{\left\langle\left(v_{\min } B_{S, \min }\right)^{2}\right\rangle}$ were calculated from 1 minute high resolution Omni solar wind parameter files. All other coupling functions were computed using the hourly averaged solar wind values computed from low resolution Omni solar wind parameter files. As standard high resolution Omni data is not always generated from the same spacecraft as the concurrent low resolution Omni file, spacecraft specific files were used to match high time resolution and low time resolution data from the same spacecraft to assure a valid comparison. Note that the widely used, sophisticated "leaky rectifier" functions $E_{W A V}$ and $d \Phi_{M P} / d t$ have better correlations with those functions which incorporate information about solar wind variability within a given

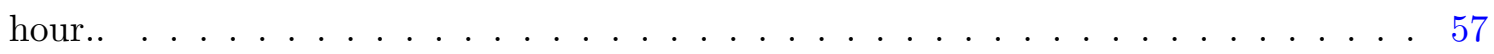

2.3 Table 4 from Newell et al. (2007). The original caption reads: "For example, Dst is best predicted by integrating over 72 hours of IMF data, with the hour n-hours previous to the present receiving relative weightof $0.95 \mathrm{n}$. Kp and cusp latitude had to be treated somewhat differently." . . . . . . . . . . . . . . 58 
2.4 The percent variance (i.e. squared correlation) of geomagnetic indices predicted by the solar wind coupling functions listed using all available data from 1973 - 2015 Omni databases. The functions $<v_{\min } B_{S, \min }>$ and $\sqrt{<\left(v_{\min } B_{S, \min }\right)^{2}>}$ were calculated from 1 minute high resolution Omni solar wind parameter files. All other coupling functions were computed using the hourly averaged solar wind values computed from low resolution Omni solar wind parameter files. As standard high resolution Omni data is not always generated from the same spacecraft as the concurrent low resolution Omni file, spacecraft specific files were used to match high time resolution and low time resolution data from the same spacecraft to assure a valid comparison. Coupling functions for $\mathrm{AE}, \mathrm{AU}$, and $\mathrm{AL}$ were correlated to the 3 hour weighted average according, weighted as described in Newell et al. (2007). $Q_{D_{S T}}$ is the injection function for the $D_{S T}$ model of Burton et al. (1975) using the original parameters from the same paper. Note that the RMS value of 1-minute $v B_{S}$ is the best predictor of $D_{S T}$ "injection", with other functions considering SW variability also performing well. For other indices, Newell's coupling function, $d \Phi_{M P} / d t$, derived empirically to match these indices, is the best predictor. However, functions considering SW variability outperform all other functions. Note also that the RMS value of 1-minute $v B_{S}$ out performs the mean value of 1 -minute $v B_{S}$ as an index predictor in all cases. 58 
2.5 The parameters for a model in the style of Burton et al. (1975) fit using simulated annealing on all data for the years 1963 through April 2016. Uncertainties were determined by repeating model fits separately for even and odd year data. The shaded model fits were produced using only data for hours when the vector standard deviation of interplanetary magnetic field (IMF) was less than half the magnitude of the IMF in the GSM y-z plane, e.g. $B_{T}>2 \sigma_{v} e c B$. Note that selection for reduced relative IMF variability produced a less "leaky" rectifier function and move exponents for density, n, and SW speed, v, closer to unity while barely changing the exponent for $B_{T}$. On the last 2 rows, model fit was produced where the vector IMF standard deviation is included. Note that the residual error is reduced while optimizing fewer free parameters. Each fit was produced twice, once minimizing RMS error (LSQ), and once minimizing the least absolute deviation (LAD). Residual errors are RMS error and mean absolute error for the LSQ and LAD cases, respectively. . . . . . . . 59

4.1 The respective cross-correlation coefficient magnitudes and percent of variation explained for ABI in the years 2000 through 2009 inclusive. The indices were processed appropriately to ensure that all logarithms receive positive input. In the case of storm-time magnetic depression, those with a "neg" subscript were set to zero when a positive index value was encountered. Note that pressure corrected Dcx* approaches the explanatory capability of of the K-family indices for ABI, as shown by highlighted examples. The "A" subscript indicates that the pressure correction found for Dcx by Asikainen et al. (2010) was used. . . . . . . . . . . . . . . . . 60

4.2 The respective cross-correlation coefficient magnitudes and percent of variation explained for $2^{o}$ binned ABI by median values of the index logarithms shown the years 2000 through 2009 inclusive. The indices were processed appropriately to ensure that all logarithms receive positive input. In the case of storm-time magnetic depression, index values were set to zero when a positive index value was encountered. Note that k-family indices have lower correlations with ABI than storm depression indices, with pressure corrected Dcx* exceeding the $99 \%$ level, as shown by highlighted examples. The "A" subscript indicates that the pressure correction found for Dcx by Asikainen et al. (2010) was used. . . . . . . . . . . . . . . . . . 61 


\section{List of Figures}

1.1 Typical plasma parameters for the solar wind in Earth's vicinity are shown along with those associated with other plasma regions of geospace. From Borovsky and

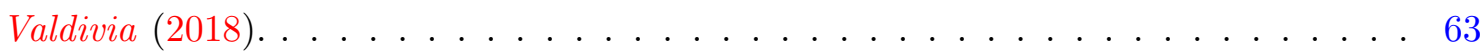

1.2 The magnetosphere is here shown in cross-section. . . . . . . . . . . . . 64

1.3 The magnetosphere is here shown with major current systems labeled. . . . . . . . 65

1.4 The SW magnetic field magnitude distribution and statistics for the years 1965 through 2016. Obtained from omniweb.gsfc.nasa.gov. . . . . . . . . . 66

1.5 The SW magnetic field GSM z-component (parallel to Earths magnetic dipole axis) distribution and statistics for the years 1965 through 2016. Obtained from omni-

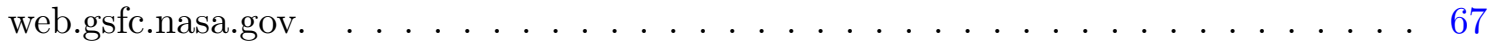

1.6 The SW speed distribution and statistics for the years 1965 through 2016. Obtained from omniweb.gsfc.nasa.gov. . . . . . . . . . . . . . . 68

1.7 The SW proton temperature distribution and statistics for the years 1965 through 2016. Obtained from omniweb.gsfc.nasa.gov. . . . . . . . . . . . . . . 69

1.8 The SW density distribution and statistics for the years 1965 through 2016. Obtained from omniweb.gsfc.nasa.gov. . . . . . . . . . . . . . . . . 70

1.9 The SW dynamic (ram) pressure distribution and statistics for the years 1965 through 2016. Obtained from omniweb.gsfc.nasa.gov. . . . . . . . . . . . . 71 
1.10 A schematic drawing of rolled-up Kelvin-Helmholtz vortices at the dusk-flank magnetopause is shown. The streamline pattern (black lines) and total (magnetic plus plasma) pressure and density (red, dense; blue, tenuous) distributions, when viewed in the vortex rest frame. The sub-solar region is to the left. From supplementary figure 4 of Kavosi and Raeder (2015). . . . . . . . . . . . . . . . . . . 72

1.11 The typical arangement of field aligned currents for relatively undisturbed times $(A L<100 n T)$. The R0 currents are the small system of currents near the pole. The R1 currents are the inner (poleward) ring of currents. The R2 currents are the outer (equaterward) ring of currents. The center is $90^{\circ}$ magnetic latitude (i.e. the northern magnetic pole). The outer edge is the $60^{\circ}$ magnetic latitude. The numbers around the outer edge specify magnetic local time (MLT). From Iijima and Potemra

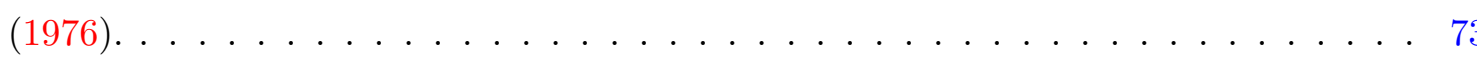

1.12 A simplified schematic of the auroral electrojet currents in the auroral oval. Hall currents are represented by dotted arrows and electric fields by solid arrows. From figure 2 in Johnsen (2013). . . . . . . . . . . . . . . . 74

1.13 The locations of magnetometers used in the calculation of $D_{S T}$ are shown. The bold line represents the magnetic equator. From (http://wdc.kugi.kyoto-u.ac.jp) . . . . . . 75

1.14 The locations of magnetometers used in the calculation of Sym/H and ASY/H are shown. The dotted line represents the magnetic equator. Those stations connected by solid lines represent alternate choices used in index calculations according to availability and quality of data. From (http://wdc.kugi.kyoto-u.ac.jp). . . . . . . 76

1.15 The locations of magnetometers used in the calculation of Dcx are shown. The curved line represents the magnetic equator. From (http://dcx.oulu.fi/?link=stations). . . . 77

1.16 The locations of magnetometers used in the calculation of AL, AU, and AE are shown. Plus signs represent magnetic coordinates. From (http://wdc.kugi.kyoto-

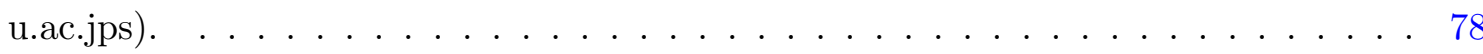


1.17 An example of DMSP sourced electron precipitation data, from a pass over the Southern pole on Dec. 12, 1978, used to produce ABI, taken from Gussenhoven et al. (1983), is shown with precipitation boundaries used marked by solid vertical lines. Electon flux, in $\left(\mathrm{cm}^{2} s s r\right)^{-1}$, is on the bottom. Energy, in $\mathrm{KeV} /\left(\mathrm{cm}^{2} \mathrm{srs}\right)$, is shown in the middle. The top pannel shows average electron energy in KeV . . . . . . 79

1.18 The magnetic influences of different magnetospheric current systems are shown, with electric currents and magnetic fields represented by arrows on diagrams in the lefthand and right-hand columns, respectively. (a) shows the effect of magnetopause currents. (b) shows the effect of symmetric ring currents. (c) shows the effect of cross-tail current. (d) shows the effect of R1 FAC. (e) shows the effect of partial ring currents their R2 FAC closure. From Kamide and Maltsev (2007). . . . . . . . . . . 80

1.19 The flux in the inner magnetosphere, denoted by $S$, and the outer magnetosphere, denoted by $F$ used by to calculate the approximate effect of cross-tail currents upon storm-time magnetic depression by Maltsev and his collaborators, is shown. From

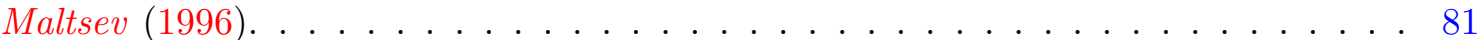

1.20 This diagram shows the general pattern of the substorm current wedge upon the disruption of the tail current sheet during a substorm. From McPherron et al. (1973). 82

1.21 An example of the Auroral Lower (AL) index of an interval with substorm activity, produced using high resolution Omni, is shown. The rapid negative excursion of AL during the substorm expansion is clearly visible. . . . . . . . . . . . . . 83

2.1 An example plot of 1 hour of solar wind electric field, $-v B_{Z}$, taken from the high resolution Omni database, is shown at 1-minute cadence. The black and blue horizontal lines represent the mean values for the hour, from this data and the low resolution Omni database, respectively. One can see that The mean values are negative and would be set to zero if $v B_{S}$ were calculated for the hour. Conversely, the red horizontal line represents the avarage value of rectified $v B_{S}$, showing that energy and flux should transfer from the solar wind during this interval, assiming $v B_{S}$ is a good measure of solar wind magnetosphere energy transfer. . . . . . . . . . . . . . 84 
2.2 Statistical mutual dependencies of solar wind parameters are shown. (a) shows the relation of a widely use $\epsilon$ parameter Perreault and Akasofu (1978) to rectified SW dawn-dusk electric field. (b) shows the relation of $\epsilon$ to IMF $B_{Z}$. (c) shows the relation between IMF magnetic field in the GSM X-Y plane to IMF $B_{Z}$. (d) shows the relation between SW speed and IMF $B_{Z}$. (e) shows the relation between SW density and IMF $B_{Z}$. (f) shows the relation between SW speed and IMF magnitude. From figure 7 in Maltsev and Rezhenov (2003). . . . . . . . . . . . . . . . 85

2.3 Statistical mutual dependencies of solar wind parameters are shown. (g) shows the relation between rectified SW dawn-dusk electric field $\left(v B_{S}\right)$ and IMF $B_{Z}$. (h) shows the relation between SW density and IMF magnitude. (i) shows the relation between SW density and SW flow speed. (j) shows the relation between IMF magnitude and SW flow speed. (k) shows the relation between rectified SW dawn-dusk electric field $\left(v B_{S}\right)$ and SW flow speed. (l) shows the relation between $\epsilon$ and SW flow speed. From figure 7 in Maltsev and Rezhenov (2003). . . . . . . . . . . . . . . 86

3.1 The impulse response of DST with respect to vBs. The horizontal axis represents hours of lag time. The vertical axis represents the response of DST (nT) per unit of rectified solar wind electric field $(\mathrm{mV} / \mathrm{m}) \ldots \ldots \ldots \ldots$. . . . . . . . 87

3.2 The impulse response of DST with respect to solar wind dynamic pressure. The horizontal axis represents hours of lag time. The vertical axis represents the response of $\operatorname{DST}(\mathrm{nT})$ per square rooted unit of pressure $(\mathrm{nPa}) \ldots \ldots \ldots$

3.3 The impulse response of DST with respect to $v|B y|$. The horizontal axis represents hours of lag time. The vertical axis represents the response of DST (nT) per unit of solar wind electric field $(\mathrm{mV} / \mathrm{m}) . \ldots \ldots \ldots \ldots \ldots$

3.4 The impulse response of DST with respect to $\mathrm{v} \sigma_{B}$. The horizontal axis represents hours of lag time. The vertical axis represents the response of DST (nT) per unit of solar wind electric field variance $(\mathrm{mV} / \mathrm{m}) \ldots \ldots \ldots \ldots$. . . . . . . . . 90

3.5 The impulse response of $\mathrm{Sym} / \mathrm{H}$ with respect to $v B_{S}$ in the high $\mathrm{SW}$ density case. . 91

3.6 The impulse response of $\mathrm{Sym} / \mathrm{H}$ with respect to $v B_{S}$ in the low $\mathrm{SW}$ density case. . . 92

3.7 The impulse response of $\mathrm{SYM} / \mathrm{H}$ with respect to $\sqrt{(} P) \ldots \ldots \ldots$

3.8 The impulse response of AL with respect to $v B_{S}$ in the high SW density case. . . . . 94 
3.9 The impulse response of AL with respect to $v B_{S}$ in the low $\mathrm{SW}$ density case. . . . . 95

3.10 The impulse response of $\mathrm{H}(\mathrm{ABI})$, the storm-time depression predicted by the theoretical formula of Maltsev and his collaborators, with respect to $v B_{S}$. It can be seen that the $\mathrm{ABI}$ data set is noisy, resulting in excessive high frequency noise in this IRF

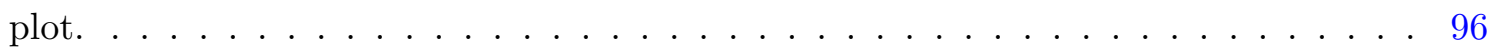

3.11 The impulse response of $\mathrm{H}(\mathrm{ABI})$ with respect to $v B_{S}$ is shown, where the coefficient are smoothed by averaging each with its nearest neighbors in lag time. . . . . . . . 97

3.12 The impulse response of $\mathrm{Sym} / \mathrm{H}$ with respect to $v B_{S}$ is shown for the case where $\mathrm{AL}$ was included as an additional driver variable. Note the attenuation of the second

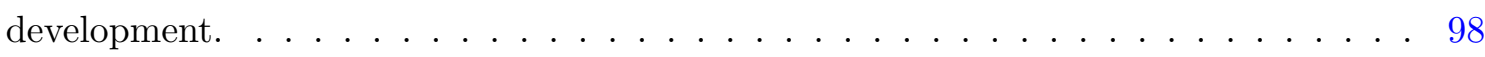

3.13 The impulse response of Sym/H with respect to AL. Note that, although this variable is successful at removing the 2nd development in figure 3.12, the error bars are very large in comparison to the respective IRF coefficients. . . . . . . . . . . . . . . 99

3.14 The impulse response of $\mathrm{Sym} / \mathrm{H}$ with respect to $v B_{S}$ is shown for the case where $\mathrm{AL}$ was not included as an additional driver variable for comparison with figure 3.12 . Note the 2 nd development at the lag time of $5-6$ hours. . . . . . . . . . . 100

3.15 The impulse response of $\mathrm{Sym} / \mathrm{H}$ with respect to $v B_{S}$ is shown for the case where $\delta A L$ was included as an additional driver variable. Note the attenuation of the second development. . . . . . . . . . . . . . . . . . . 101

3.16 The impulse response of $\mathrm{Sym} / \mathrm{H}$ with respect to negative $\delta A L$ is shown. A positive (negative) value means the unloading produces a strengthening (weakening) of the index at that lag time. . . . . . . . . . . . . . . . . 102

3.17 The impulse response of SMR with respect to $\mathrm{v} B_{S}$. The IRF for this index is similar

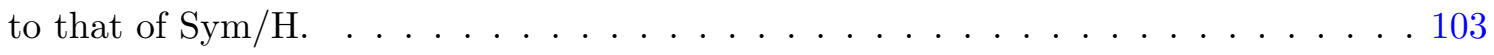

3.18 The impulse response of midnight sector SMR00 with respect to $\mathrm{v} B_{S} . \ldots 104$

3.19 The impulse response of dawn sector SMR06 with respect to $\mathrm{v} B_{S} \ldots \ldots 5$

3.20 The impulse response of noon sector SMR12 with respect to $\mathrm{v} B_{S}$. . . . . . . 106

3.21 The impulse response of dusk sector SMR18 with respect to $\mathrm{v} B_{S} \ldots \ldots$. . . . . 107

3.22 The impulse response of midnight sector SMR00 with respect to $\sqrt{P} \ldots \ldots$. . . . 108

3.23 The impulse response of dawn sector SMR06 with respect to $\sqrt{P} \ldots \ldots$. . . . . 109 
3.24 The impulse response of noon sector SMR12 with respect to $\sqrt{P} \ldots \ldots$. . . . . 110

3.25 The impulse response of dusk sector SMR18 with respect to $\sqrt{P} \ldots \ldots 11$

3.26 The impulse response of SMR with respect to $\mathrm{v} B_{S}$, where the negative changes in AL are added as an additional regression variable. . . . . . . . . . . . . . . 112

3.27 The impulse response of midnight sector SMR00 with respect to $\mathrm{v} B_{S}$, where the negative changes in $\mathrm{AL}$ are added as an additional regression variable. . . . . . . . . 113

3.28 The impulse response of dawn sector SMR06 with respect to $\mathrm{v} B_{S}$, where the negative changes in AL are added as an additional regression variable. . . . . . . . . . . . . 114

3.29 The impulse response of noon sector SMR12 with respect to $\mathrm{v} B_{S}$, where the negative changes in AL are added as an additional regression variable. . . . . . . . . . . . 115

3.30 The impulse response of dusk sector SMR18 with respect to $\mathrm{v} B_{S}$, where the negative changes in AL are added as an additional regression variable. . . . . . . . . . . 116

3.31 The impulse response of midnight sector SMR00 with respect to negative $d(A L) / d t$ to represent the substorm unloading process. A positive (negative) value means the unloading produces a strengthening (weakening) of the index. . . . . . . . 117

3.32 The impulse response of dawn sector SMR06 with respect to negative $d(A L) / d t$ to represent the substorm unloading process. A positive (negative) value means the unloading produces a strengthening (weakening) of the index. . . . . . . . . . 118

3.33 The impulse response of noon sector SMR12 with respect to negative $d(A L) / d t$ to represent the substorm unloading process. A positive (negative) value means the unloading produces a strengthening (weakening) of the index. . . . . . . . . . . 119

3.34 The impulse response of dusk sector SMR18 with respect to negative $d(A L) / d t$ to represent the substorm unloading process. A positive (negative) value means the unloading produces a strengthening (weakening) of the index. . . . . . . . . . . 120

3.35 The impulse response of modeled $D_{S T}$ separated by storm phase with respect to $\mathrm{v} B_{S}$ as found in Vassiliadis et al. (1999). The dashed (solid) lines represent weak (strong) driving. The heavy line is averaged for all conditions. We draw attention to the the strongly driven late commencement case. Could this be the a signature of behavior of midnight and dawn sectors in response to substorm unloading? . . . . 121 
3.36 This schematic diagram from Baumjohann (1986) shows the conventional understanding of solar wind energy flow and dissipation in the magnetosphere. Note that $D_{S T}$ is considered to be dependent on ring current. . . . . . . . . . . . . 122

3.37 This schematic diagram from Baumjohann (1986) of solar wind energy flow and dissipation in the magnetosphere has been modified (in red) to add the effect of cross tail currents. Although several studies have found non-trivial contributions of tail current to storm-time depression, many in the field continue to view $D_{S T}$ and its clones as primarily a ring current proxy as in figure 3.36 . . . . . . . . . . 123

3.38 This schematic diagram from Baumjohann (1986) of solar wind energy flow and dissipation in the magnetosphere has been further modified (in red) from figure 3.37 to add the time delayed contribution of oxygen ions liberated from the ionosphere by substorm events. In this view, supported by the impulse response studies presented in this chapter, the ring current contribution develops after the initial tail contribution.124

3.39 An example IRF from the model, schematically shown in the previous figure. This figure was produced as follows. (1) An exponential decay series representing the hypothesized tail current contribution to geomagnetic storm-time depression was produced according to $Y_{1}(t)=-e^{-t / \tau_{1}}$, where $\tau_{1}$ is 2 hours and $t$ is lag time. (2) The rate of decline in magnitude for $y_{1}$, assumed to be partly due to substorm activity, is $\dot{Y}_{1}=-Y_{1} / \tau_{1}$. This is assumed to be related to $\mathrm{O}+$ ion injection into the ring current, $Q_{O+}=-\kappa \dot{Y}_{1}$. (3) The injected ring current decays at the longer timescale of $\tau_{2}=17.3$ hours, with injection delayed by $3-4.5$ hours after the contributing tail current decay, leading to the evolution equation $\Delta Y_{2, t_{m}}=\sum_{n=1}^{N}\left(Q\left(t_{m}-d_{n}\right) / N\right)-$ $Y_{2, t(m-1) / \tau_{2}}$, where $d_{1}=3$ hours and $d_{N}=4.5$ hours. (4) The overall sum, $Y=Y_{1}+$ $Y_{2}$, is plotted versus IRF lag time for values of $\kappa=0.1$ (minimal 2nd development) to $\kappa=0.4$ (prominent 2 nd development) in increments of $0.05 \ldots \ldots \ldots$

4.1 The average latitudinal range of the auroral oval, as determined from all-sky-imager cameras, near local midnight parametrized by $\mathrm{Kp}$ is shown. It is seen that the equatorward location moves to lower latitudes as activity increases. Feldstein and

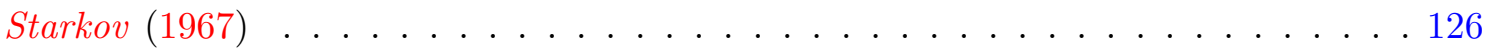


4.2 An example from Thomsen (2004) showing that the quasi-logarithmic Kp index closely relates to ABI, which tracks the measured latitude of the equatorward auroral boundary as determined from electron precipitation. . . . . . . . . . . . . 127

4.3 The ap index (linear equivalent to the quasi-logarithmic Kp index) plus one is shown on a logarithmic scale versus ABI for years 2000-2009. For robustness, median values are plotted with mean deviation from the median, computed separately for values above and below the median, used for error bars. . . . . . . . . . . . . . . 128

4.4 The am index (similar to ap, but based on a more global set of observatories and continuous in magnitude) plus one is shown on a logarithmic scale versus ABI for years 2000-2009. Note that the logarithm of am appears more linear with respect to $\mathrm{ABI}$ on this data set, confirming claims that am is a higher quality index than Kp. For robustness, median values are plotted with mean deviation from the median, computed separately for values above and below the median, used for error bars. . . 129

4.5 The midnight sector am00 index plus one is shown on a logarithmic scale versus ABI for years 2000-2009. For robustness, median values are plotted with mean deviation from the median, computed separately for values above and below the median, used for error bars. . . . . . . . . . . . . . . . . . . . 130

4.6 The dawn sector am06 index plus one is shown on a logarithmic scale versus ABI for years 2000-2009. For robustness, median values are plotted with mean deviation from the median, computed separately for values above and below the median, used

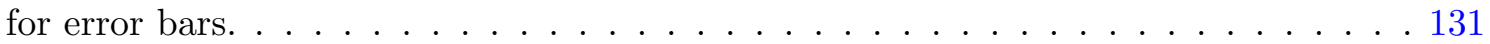

4.7 The noon sector am12 index plus one is shown on a logarithmic scale versus ABI for years 2000-2009. For robustness, median values are plotted with mean deviation from the median, computed separately for values above and below the median, used for error bars. . . . . . . . . . . . . . . . . . . . . 132

4.8 The dusk sector am18 index plus one is shown on a logarithmic scale versus ABI for years 2000-2009. For robustness, median values are plotted with mean deviation from the median, computed separately for values above and below the median, used

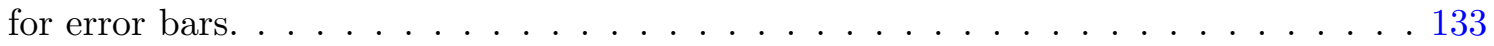


4.9 The $\alpha 15$ index (similar to am, but available for 15 minute time windows instead of the typical 3 hour windows use for the K-family of indices) plus one is shown on a logarithmic scale versus ABI for years 2000-2009. For robustness, median values are plotted with mean deviation from the median, computed separately for values above and below the median, used for error bars. . . . . . . . . . . . . . 134

4.10 The magnitude of $D_{S T}$ index, for times when $D_{S T}<0$, plus one is shown on a logarithmic scale versus ABI for years 2000-2009. For robustness, median values are plotted with mean deviation from the median, computed separately for values above and below the median, used for error bars. Note that $D_{S T}$ is mostly linear on this scaling, as are the K-family indices. . . . . . . . . . . . . . 135

4.11 The magnitude of Dcx index, for times when $D c x<0$, plus one is shown on a logarithmic scale versus ABI for years 2000-2009. For robustness, median values are plotted with mean deviation from the median, computed separately for values above and below the median, used for error bars. Note that Dcx is more linear on this scaling than $D_{S T}$, giving support to claims that Dcx is a corrected version of $D_{S T}$. 136

4.12 The magnitude of pressure corrected $D_{S T}^{*}$ index, for times when $D_{S T}^{*}<0$, plus one is shown on a logarithmic scale versus ABI for years 2000-2009. For robustness, median values are plotted with mean deviation from the median, computed separately for values above and below the median, used for error bars. Note that $D_{S T}$ is mostly linear on this scaling, as are the K-family indices. . . . . . . . . . . . 137

4.13 The magnitude of pressure corrected Dcx* index, for times when $D c x *<0$, plus one is shown on a logarithmic scale versus ABI for years 2000-2009. For robustness, median values are plotted with mean deviation from the median, computed separately for values above and below the median, used for error bars. Note that Dcx is more linear on this scaling than $D_{S T}$, giving support to claims that Dcx is a corrected version of $D_{S T}$. The theoretical tail contribution of tail currents derived in Maltsev (2004) is shown in red, with solid and dashed lines for cases with and without a ground induction factor of 1.3 , respectively. The empirically obtained formula for tail current contribution according to Asikainen et al. (2010) is shown in blue. . . . 138 
4.14 The magnitude of pressure corrected Dcx* index, using the Dcx specific pressure correction obtained in Asikainen et al. (2010), for times when $D c x *<0$, plus one is shown on a logarithmic scale versus ABI for years 2000-2009. For robustness, median values are plotted with mean deviation from the median, computed separately for values above and below the median, used for error bars. Note that Dcx is more linear on this scaling than $D_{S T}$, giving support to claims that Dcx is a corrected version of $D_{S T}$. The theoretical tail contribution of tail currents derived in Maltsev (2004) is shown in red, with solid and dashed lines for cases with and without a ground induction factor of 1.3, respectively. The empirically obtained formula for tail current contribution according to Asikainen et al. (2010) is shown in blue. . . . 139

4.15 The magnitude of 10 minute average SMR index, for times when $S M R<0$, plus one is shown on a logarithmic scale versus ABI for years 2000-2009. For robustness, median values are plotted with mean deviation from the median, computed separately for values above and below the median, used for error bars. Note that SMR is less linear on this scaling than $D c x . \ldots \ldots \ldots \ldots$

4.16 The magnitude of 10 minute average midnight sector SMR00 index, for times when $S M R 00<0$, plus one is shown on a logarithmic scale versus ABI for years 20002009. For robustness, median values are plotted with mean deviation from the median, computed separately for values above and below the median, used for error

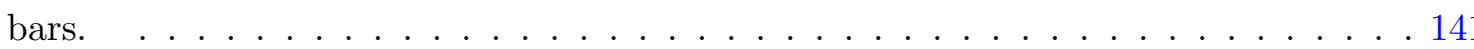

4.17 The magnitude of 10 minute average dawn sector SMR06 index, for times when $S M R 06<0$, plus one is shown on a logarithmic scale versus ABI for years 20002009. For robustness, median values are plotted with mean deviation from the median, computed separately for values above and below the median, used for error

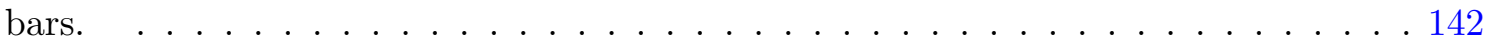

4.18 The magnitude of 10 minute average noon sector SMR12 index, for times when $S M R 06<0$, plus one is shown on a logarithmic scale versus ABI for years 20002009. For robustness, median values are plotted with mean deviation from the median, computed separately for values above and below the median, used for error

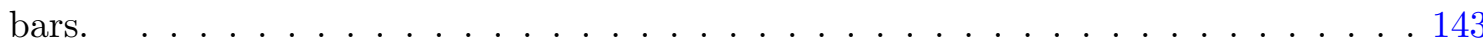


4.19 The magnitude of 10 minute average dusk sector SMR12 index, for times when $S M R 06<0$, plus one is shown on a logarithmic scale versus ABI for years 20002009. For robustness, median values are plotted with mean deviation from the median, computed separately for values above and below the median, used for error

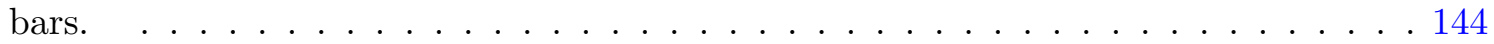

4.20 The field aligned currents for the northern hemisphere for a typical cluster when Dcx averages to $0 \mathrm{nT}$ is shown. Note the contracted, weak R1 and R2 currents, consistent with the tail dominated storm picture. Red represents upward current and green represents downward currents. . . . . . . . . . . . . . . . 145

4.21 The field aligned currents for the northern hemisphere for a typical cluster when Dcx averages to $-25 \mathrm{nT}$ is shown. Note that R1 and R2 currents have expanded significantly from the Dcx $=0 \mathrm{nT}$ case, consistent with the tail dominated storm picture. Red represents upward current and green represents downward currents. . . 146

4.22 The field aligned currents for the northern hemisphere for a typical cluster when Dcx averages to $-49 \mathrm{nT}$ is shown. Note that R1 and R2 currents have expanded significantly from the Dcx $=-25 \mathrm{nT}$ case, consistent with the tail dominated storm picture. Red represents upward current and green represents downward currents. . . 147

4.23 The field aligned currents for the northern hemisphere for a typical cluster when Dcx averages to $-75 \mathrm{nT}$ is shown. Note that R1 and R2 currents have expanded significantly from the Dcx $=-49 \mathrm{nT}$ case, consistent with the tail dominated storm picture. Red represents upward current and green represents downward currents. . . 148

4.24 The field aligned currents for the northern hemisphere for a typical cluster when Dcx averages to $-104 \mathrm{nT}$ is shown. Note that R1 and R2 currents have expanded significantly from the Dcx $=-75 \mathrm{nT}$ case, consistent with the tail dominated storm picture. Red represents upward current and green represents downward currents. . . 149

4.25 The field aligned currents for the northern hemisphere for a typical cluster when Dcx averages to $-159 \mathrm{nT}$ is shown. Note that R1 and R2 currents have expanded significantly from the Dcx $=-104 \mathrm{nT}$ case, consistent with the tail dominated storm picture. Red represents upward current and green represents downward currents. . . 150 
4.26 Plots of FAC intensity in $\mu A / m^{2}$ along the 3 meridia used for $\mathrm{R} 2 \mathrm{FAC}$ location identification corresponding to figure 4.20. The automatically identified R2 peaks are marked in red. . . . . . . . . . . . . . . . . . 151

4.27 The 6 points identified in figure 4.26 as most likely R2 FAC peaks are marked. The circle shown was fit to these points and represents the most likely equatorward boundary of the auroral oval. . . . . . . . . . . . . . . . . 152

4.28 Plots of FAC intensity in $\mu A / m^{2}$ along the 3 meridia used for $\mathrm{R} 2 \mathrm{FAC}$ location identification corresponding to figure 4.21. The automatically identified R2 peaks are marked in red. . . . . . . . . . . . . . . . . . . . 153

4.29 The 6 points identified in figure 4.28 as most likely R2 FAC peaks are marked. The circle shown was fit to these points and represents the most likely equatorward boundary of the auroral oval. . . . . . . . . . . . . . . . 154

4.30 Plots of FAC intensity in $\mu \mathrm{A} / \mathrm{m}^{2}$ along the 3 meridia used for $\mathrm{R} 2 \mathrm{FAC}$ location identification corresponding to figure 4.22. The automatically identified R2 peaks are marked in red. . . . . . . . . . . . . . . . . . . 155

4.31 The 6 points identified in figure 4.30 as most likely R2 FAC peaks are marked. The circle shown was fit to these points and represents the most likely equatorward boundary of the auroral oval. . . . . . . . . . . . . . 156

4.32 Plots of FAC intensity in $\mu A / m^{2}$ along the 3 meridia used for $\mathrm{R} 2 \mathrm{FAC}$ location identification corresponding to figure 4.23. The automatically identified R2 peaks are marked in red. . . . . . . . . . . . . . . . . . 157

4.33 The 6 points identified in figure 4.32 as most likely R2 FAC peaks are marked. The circle shown was fit to these points and represents the most likely equatorward boundary of the auroral oval. . . . . . . . . . . . . . 158

4.34 Plots of FAC intensity in $\mu \mathrm{A} / \mathrm{m}^{2}$ along the 3 meridia used for $\mathrm{R} 2 \mathrm{FAC}$ location identification corresponding to figure 4.24. The automatically identified R2 peaks are marked in red. . . . . . . . . . . . . . . . . . . 159

4.35 The 6 points identified in figure 4.34 as most likely R2 FAC peaks are marked. The circle shown was fit to these points and represents the most likely equatorward boundary of the auroral oval. . . . . . . . . . . . . . . 160 
4.36 Plots of FAC intensity in $\mu A / \mathrm{m}^{2}$ along the 3 meridia used for $\mathrm{R} 2 \mathrm{FAC}$ location identification corresponding to figure 4.25. The automatically identified R2 peaks are marked in red. . . . . . . . . . . . . . . . . . 161

4.37 The 6 points identified in figure 4.36 as most likely R2 FAC peaks are marked. The circle shown was fit to these points and represents the most likely equatorward boundary of the auroral oval. . . . . . . . . . . . . . . . . . . 162

4.38 The median values of Dcx are plotted versus the theoretical cross tail current contribution $H(\theta)$, where $\theta$ is the radius of the auroral oval in degrees as determined from AMPERE data. Error bars represent the mean absolute deviation. . . . . . . . . . 163

4.39 The median values of pressure corrected Dcx* are plotted versus the theoretical cross tail current contribution $H(\theta)$, where $\theta$ is the radius of the auroral oval in degrees as determined from AMPERE data. Error bars represent the mean absolute deviation. A line of slope 1 is added to guide the eye . . . . . . . . . . . . . . 164 


\section{Chapter I}

\section{Introduction}

\section{A. The Magnetosphere}

In the region of geospace surrounding Earth, the region where Earth's magnetic field dominates is referred to as the magnetosphere Parks (1991). As solar wind plasma (see figure 1.1) rushes past the magnetosphere at supersonic speeds, it interacts with the magnetosphere, compressing it on the dayside and stretching it into the magnetotail on the nightside. As Earth's core generates a mostly dipolar magnetic field, such distortions in field shape can only arise from electric currents produced by these interactions. The typical structure of the magnetosphere and its currents is shown in figures 1.2 and 1.3. In the following sections, we will describe the major features of this system

\section{The Solar Wind}

The solar wind (SW) in the Earth's orbital zone is a supersonic flow of plasma from the Sun. As the Sun is a highly dynamic environment, the parameters of the SW, including its embedded interplanetary magnetic field (IMF), density, velocity, composition, and temperature all vary with time. See figures 1.4 through 1.9 for SW variable statistics generated from the hourly SW values for 1965-2016 using NASA's Omni SW database. From Earth's perspective, the SW typically flows in a direction offset $\sim 4$ degrees from the Earth-Sun line. This is due to the orbital motion of Earth about the Sun.

As the supersonic SW flows past the magnetosphere, a bow shock forms. This creates a slower 
region of compressed SW plasma, bordering the magnetosphere, called the magnetosheath. This magnetosheath plasma is the plasma that directly interacts with the magnetosphere.

\section{The Magnetopause and Cusp}

The Magnetopause serves as a magnetic boundary, it sustains electric currents which serve to isolate the magnetospheric magnetic field from the SW IMF. The position of the dayside magnetopause is controlled largely by a pressure balance where ram pressure from the SW is balanced by magnetic pressure inside the magnetosphere.

The SW acts as a driver of magnetospheric phenomena through processes at the magnetopause by which the SW transfers energy and plasma to the magnetosphere. The transfer process thought to be most important is magnetic reconnection. This occurs when magnetic field lines in the magnetosheath have components anti-parallel to magnetospheric field lines. The topology changes, causing previously closed field lines to become connected to the IMF. These open field lines are then convected to the nightside magnetotail. This process occurs most prominently when the IMF has a strongly southward component. It also has an effect on magnetopause position as day-side reconnection tends to erode magnetic flux from the dayside magnetosphere.

A less dominant (and more poorly quantified and understood) effect occurring at the magnetopause is viscous energy transfer. This is a transfer of fluid energy from the SW to the magnetosphere through mechanisms such as the Kelvin-Helmholtz instability, which is driven by velocity shear of plasma flows over a magnetopause (see figure 1.10).

The cusp regions are high latitude regions of depressed magnetic field near the poles with pooling of magnetosheath plasma, but displaced toward the dayside, where recently reconnected open field lines are swept toward the magnetotail. The positions of the cusps vary in response to SW ram pressure and reconnection.

\section{The Magnetotail}

The magnetotail is the nightside region of the magnetosphere where the field lines are stretched anti-sunward by SW convection. The magnetotail is composed of northern and southern lobes composed of open field lines which accumulate just beyond the cusp region. Observations in the far 


\section{CHAPTER I. INTRODUCTION}

tail region are scant, but arguments based on convection times of field lines and SW speed indicate that it is somewhere between 100 and 1000 Earth radii (RE) long.

As the two lobes have anti-parallel magnetic fields, the are separated by a current sheet which closes through the magnetopause. Reconnection occurs in this current sheet, returning open field lines to a closed topology, where the are part of the plasma sheet. Magnetic tension then serves to convect these field lines Earthward, eventually returning them to the dipole like region of the magnetosphere. There is some lack of precision in the definition of the tail region. Some restrict it to strictly refer only to the open field lines. Others would include those closed field lines which have not yet returned to a dipolar configuration as part of the tail.

\section{The Inner Magnetosphere}

The inner magnetosphere is that region close enough to Earth to have primarily dipolar character in its magnetic field configuration, and bounded by the ionized ionosphere region of the upper atmosphere. It contains features several important current systems which are relevant in the study of geomagnetic phenomena and aurorae.

The ring current has two parts. The symmetric ring current is composed of trapped ions which drift westward around the Earth, causing the magnetic field on the ground at low and mid latitudes to weaken. The partial ring current, like its symmetric counterpart, also is composed of westward drifting trapped ions. However, these ions do not close around the dayside of the magnetosphere, but with the ionosphere via region 2 field aligned currents (FAC). The ring currents are not static, but vary in time. They are thought to dissipate via the processes of charge exchange with neutral atoms, losses through the magnetopause, and, for partial ring currents, via resistive dissipation in the ionosphere (though not often stated). Maltsev (2004) Ganushkina et al. (2015)

The FAC (see figure 1.11) are normally classified as region $0(\mathrm{R} 0)$, region 1 (R1), and region 2 (R2) [Iijima and Potemra (1976)]. As mentioned above R2 FAC provide closure for the partial ring current. R1 FAC are partly driven by a dynamo process [Stern (1983)] connecting the ionosphere with the interplanetary motional electric field, as field lines convect from the day-side through the open field region in the polar caps of the ionosphere surrounded by the auroral oval, from which the tail lobes emerge. R1 FAC are thus typically poleward of the R2 FAC, although this ordering can sometimes be confused at local noon and midnight locations. R0 currents are sometimes called 
NBZ currents because they are notable during periods of northward IMF. They are found in the cusp region poleward of R1 currents.

\section{The Ionosphere}

The ionosphere serves as an inner boundary of the magnetosphere. It is a partially ionized region of the upper atmosphere, conventionally approximated at a conductive sheet $110 \mathrm{~km}$ above Earth's surface. The ionization results from UV radiation from the Sun (on the dayside) and other light sources (on the nightside), from particle precipitation, and from Joule heating caused by ionospheric currents. In addition to the simple conductivity, $\sigma_{\|}$, of elementary electrodynamics, the ionosphere possesses both Hall conductivity, $\sigma_{H}$, representing the ease with which the Hall electric current flows perpendicular to both the driving electric field and magnetic field, and Pedersen conductivity, $\sigma_{P}$, representing the ease with which the current flows parallel to the driving electric field but perpendicular to the magnetic field. Ohm's law for current density then becomes $\vec{J}=\sigma_{P}\left(\overrightarrow{E_{\perp}}+\right.$

$\vec{U} \times \vec{B})+\sigma_{H} \vec{B} / B \times\left(\overrightarrow{E_{\perp}}+\vec{U} \times \vec{B}\right)+\sigma_{\|} \vec{E}_{\|}$, where $\vec{B}$ is magnetic field, $\vec{U}$ is plasma velocity, $E_{\perp}$ is electric field perpendicular to magnetic field, and $E_{\|}$is electric field parallel to magnetic field.

The anti-sunward motion of field lines through the polar ionosphere serves as to produce a duskward electric field. The auroral oval has high conductivity due to intense particle precipitation. Accordingly, the Hall currents are diverted to follow a path along the auroral oval, becoming the eastward and westward auroral electrojet currents. See figure 1.12.

\section{B. Quantifying Magnetospheric Behavior}

Ground-based observations of aurorae or magnetic disturbances from any one location do not provide much information about the global state of the magnetosphere. As studies of the magnetosphereionosphere system from the ground advanced, it became desirable to combine measurements from multiple locations in a way that ideally yields information about the global state of the magnetosphereionosphere or one or more of its constituent parts. Accordingly, a number of indices have been developed. 


\section{Storm Time Indices: $D_{S T}, \mathrm{SYM} / \mathrm{H}, \mathrm{Dcx}, E_{S T}$, and SMR}

As geomagnetic storms are known to cause a southward depression in magnetometer readings at mid-latitude and low-latitude magnetometer stations, the $D_{S T}$ index was conceived as a measure of storm strength. Early versions were sometimes constructed using a high cadence data set from stations at many local times Burton et al. (1975). However the standard form of $D_{S T}$ is constructed from only 4 ground stations (see figure 1.13 and table 1.1) at an hourly cadence. $D_{S T}$ and other indices are typically calculated by subtracting the quiet-time variation, Sq, part of the $\Delta H$ (horizontal) componant of ground magnetic field to obtain a corrected magnetic deviation, $D(T)=\Delta H(T)-S q(T)$, where quiet-time variation is determined from measured $\Delta H$ on quiet days and $T$ represents local time. The contributing stations are averaged and normalized according to $D_{S T}=D / \cos (\phi)$, where $\cos (\phi)$ is the average cosine of dipole latitudes of the contributing magnetometer stations.

Attempts have been made to correct the deficiencies of standard $D_{S T}$. The $\mathrm{SYM} / \mathrm{H}$ index is a high (1-minute) cadence version of $D_{S T}$ using 6 stations (see figure 1.14 and table 1.2). The $\mathrm{ASY} / \mathrm{H}$ index is a measure of spatial asymmetry of the storm-time magnetic depression. The chief disadvantage is that SYM/H is available for fewer years. Dcx Mursula et al. (2011) is calculated from the same stations as $D_{S T}$, or a larger superset of 17 stations since 2000 (see figure 1.15 and table 1.3), using a more careful baseline subtraction, an improved weighted average between stations, and a longer time history. $E_{S T}$ uses an Earth conductivity model to attempt the isolation of those parts of the storm-time disturbance that are due to external sources [Maus and Weidelt (2004)]. Neither Dcx nor $E_{S T}$ are widely used. In the case of Dcx, this is likely because the index is fairly new and the data is not widely available through popular distribution channels. $E_{S T}$ is not used in this dissertation because my preliminary tests suggest that the version available to the public has errors.

One of the newest sets indices in this family is the Supermag SMR Sectoral indices [Newell and Gjerloev]. The SMR index is the average of 4 sector SMR indices, SMR00, SMR06, SMR12, and SMR18, from magnetic local time (MLT) sectors centered respectively about MLTs of 0, 6, 12, and 18 hours. The SMR indices, unlike the others, do not use a fixed list of magnetic stations, but rather the entire set of stations between -50 and +50 degrees magnetic latitude. For each 
station, the northward magnetic component, $N$, is used with baseline subtracted. Each station is corrected according to its respective magnetic latitude, $N_{c o r r}=N / \cos ($ mlat $)$, and averaged with other stations in the same MLT sector to construct the sector indices.

\section{Auroral Electrojet Indices: AL, AU, and AE}

The strength of the auroral electrojets can be monitored by ground magnetometers in the auroral zone. $\mathrm{AL}, \mathrm{AU}$, and $\mathrm{AE}$ are calculated respectively from the most negative, most positive, and range of horizontal magnetic perturbations from up to 13 magnetometers (see figure 1.16) in the auroral zone. AL (auroral lower) is a measure of the westward electrojet, defined by $A L=\min \left(H_{i}\right)$, where $H_{i}$ is the $\mathrm{H}$ component for each station, i. AU (auroral upper) is a measure of the eastward flowing auroral electrojet. $\mathrm{AU}$ is defined by $A U=\max \left(H_{i}\right)$. $\mathrm{AL}$ is also a measure of the explosive energy release due to substorms if a station is proximate to the substorm current wedge. AE is the difference between $\mathrm{AU}$ and $\mathrm{AL}, A E=A U-A L$. One problem with these indices is that magnetometer data is often unavailable from the Russian sector. Another problem is that, during storm times, the auroral oval moves equatorward such that coverage in the storm time auroral zone is lacking. An attempt to rectify this shortcoming is to use all available stations that might potentially be in the auroral zone, as made available from Supermag as the SML, SMU, and SME indices to replace AL, AU, and AE, respectively [Newell and Gjerloev (2011)]. Unfortunately, the SME indices are automatically derived without human involvement. Due, as far as I know, to either baseline subtraction methodology and/or inclusion of faulty station reading in an index relying upon extrema, they suffer from unphysical jumps, often at the boundary of a month, making their use for some purposes problematic.

\section{Global Variation based Indices: Kp, ap, am, am-sector and $\alpha 15$}

One of the earliest types of geomagnetic index is the $\mathrm{K}$ index, which is a quasi-logarithmic measure of local magnetic deviation from an established daily quiet condition baseline during a 3-hour period. When $\mathrm{K}$ indices from many locations are weighted together, one gets $\mathrm{Kp}$, the planetary $\mathrm{K}$ index. The ap is the linear scaled version of $\mathrm{Kp}$ calculated from Kp with a look-up table. The am is a variant of ap calculated directly from a station network with a more global geographic distribution to provide a better measure of global activity. The am index has also been made 
available in sector contribution form [Chambodut et al. (2013)], where the 4 sectors indices, am00, am06, am12, and am18 are centered about midnight, dawn, noon, and dusk sectors. A recent attempt to make the a version of am available at a faster (15-minute) cadence has resulted in the $\alpha 15$ index [Chambodut et al. (2015)], currently available for only the years 2000 through 2009. Though global K-family indices were created without a good understanding of what they physically represent, they are currently thought to provide a fairly good proxy equatorward expansion of the auroral oval [Perrone and Franceschi (1998); Thomsen (2004)], indicative of the tail current sheet moving closer to Earth [Newell et al. (1998)].

\section{Auroral Boundary Index and the MT Index}

The Auroral Boundary Index (ABI), also called Midnight Boundary Index (MBI) is a measure of the magnetic latitude of the equatorward boundary of the Auroral oval [Madden and Gussenhoven (1990); Gussenhoven et al. (1982)]. This maps to the edge of the near tail currents in the edge of the plasma sheet, thus giving it similar meaning to Kp. The most widely available version of this index is calculated from electron particle precipitation data from DMSP satellites and is available for the years 1983-2014. The equatorward boundary of diffuse electron precipitation from the auroral oval is identified (see figure 1.17). In the ith magnetic local time (MLT), the measured magnetic latitude of the boundary, $\Lambda_{C G M}$, is first used to predict the most likely Kp value, $K p^{\prime}=\left(\Lambda_{C G M}-\Lambda_{0 i}\right) / \alpha_{i}$, where $\Lambda_{0 i}$ is the typical quiet-time value and $\alpha_{i}$ is an empirically fit parameter. Predicted Kp is then used to compute the midnight value of the index, $A B I=\Lambda_{0 M}+\alpha_{M} K p^{\prime}$, where $\Lambda_{0 M}$ is the quiet-time magnetic latitude in the midnight sector and $\alpha_{M}$ is a fit parameter. As most DMSP crossings of the auroral oval are not often at local midnight, statistical fits are used to translate a boundary determination to its most likely midnight sector value. As the auroral oval is not always a perfect oval, this leads to index errors. Accordingly, the index is distributed with data quality flags 1-3, with 1 being best and 3 being worst. Because the physical meaning of ABI is similar to $\mathrm{Kp}$, a predicted $\mathrm{Kp}$ value is also distributed with each $\mathrm{ABI}$ value.

A newer index attempting to measure the same boundary as ABI is the magnetotail (MT) index [Gvozdevsky and Sergeev (1996)]. MT is produced by attempting to detect the ion isotropy boundary using ion particle detections. In practice, this is often produced from ion particle boundaries detected by DMSP [Newell et al. (1998)]. I have verified by direct inspection of DMSP bound- 
ary data files that ion boundary locations used for MT nearly always coincide with the electron boundaries used to generate ABI.

\section{Modeling Techniques}

As shown in the preceding sections, the SW-magnetosphere-ionosphere (SMI) is a complex system. A number of approaches have been used to deal with the complexity of the SMI system. The can broadly be classified as either physics-based or empirical methods. Each approach has its own advantages and limitations. In the sections that follow, the techniques of physics-based and empirical modeling will be discussed.

\section{Physics-Based Modeling}

In physics-based modeling, one attempts to understand processes in geospace by building models that deductively use the equations of plasma physics to simulate the SMI system. Approaches range from directly treating particle motion, to using the Vlasov equations of kinetic theory, to using the fluid equations of magnetohydrodynamics. All of these approaches are under the disadvantage of requiring high-speed computing resources.

There are too many particles in the magnetosphere to simulate, so particle based approached typically represent large numbers of particles by representative super-particles, which are then assigned to particular location in the simulation. This approach is called Particle-In-Cell (PIC) simulation [Birdsall and Langdon (2004)]. PIC simulations have the advantage of including particle physics effects. However, they have the disadvantage of requiring more computational resources than other methods require.

Vlasov codes integrate the Vlasov equation from kinetic theory. In principle, this also avoids neglecting particle kinetic effects. However, since this involves 3 spatial dimensions plus 3 dimensions in velocity phase space, it requires a 6 dimensional simulation, which, like PIC codes, requires extensive computing resources [Büchner (2005)].

Magnetohydrodynamic (MHD) codes require relatively less computing resources at the expense of neglecting particle and collective effects [Tajima (2018)]. Because of this, MHD codes are widely used for realistic space plasmas, especially when real-time simulations are required. MHD equa- 
tions are derived using the assumption that the particles comprising the plasma have a Gaussian velocity distribution, which is not always true in space. Most MHD codes implement a number of approximations, such as neglecting Hall, multi-fluid, and electron inertia effects to make simulations tractable on currently available hardware. They also frequently make use of the Boris correction, which sets the speed of light artificially low in order to reduce wave speeds and thus increase allowable time steps in global models. Because of these approximations, though MHD codes are widely used and widely available, they are unable to adequately represent many of the processes in the magnetosphere.

\section{Empirical Modeling}

Empirical modeling, in contrast to the physics-based methods discussed above, inductively tries to gain an understanding through analyzing the ever growing body of data take from ground based and space based measurements. Because this typically does not require the integration of nonlinear partial differential equations, empirical models are fast and thus require much fewer computational resources than physics-based models. In the following sections, we will discuss the techniques of and approaches to empirical modeling.

\section{i. Static Versus Dynamic Models}

Static models are fit to describe a system state without regard to past states. For example, the T89 model of the magnetosphere is an empirically fit model of the magnetic field of the magnetosphere, parametrized primarily by the value of the index Kp [Tsyganenko (1989)]. Other static models attempt to predict a measure of magnetospheric state by use of solar wind state at a single (either concurrent, or with a fixed lag) time. An example would be the Papitashvili et al. (2002) model of FAC, which parametrizes FAC maps by season and concurrent interplanetary magnetic field (IMF).

Dynamic models seek to incorporate an understanding of past system state to show how the system evolves over time. Dynamic models can include such methods as recurrent artificial neural networks [e.g. Wu and Lundstedt (1996)], differential equation models which show system time evolution [e.g. Burton et al. (1975)]. Dynamic models can be attactive to those seeking insight into underlying physical processes, or alternately allow conceptions of the underlying physical system to be fit to observed data. In the Burton et al. (1975) model, for instance, the parameters of an 
evolution equation, originally intended to model growth and decay of the ring current magnetic impact on the ground, are fit to solar wind and $D_{S T}$ data for a series of geomagnetic storms (see chapter 2 for details).

\section{ii. Statistical Regression}

Statistical regression techniques seek to fit a continuous numeric output value to a set of input values. As a family of techniques, there are many choices to be made, such as: Shall the outputs be a linear or nonlinear function of the input values? Linear fits are most common. If nonlinear functions are desired, the choices of polynomial, trigonometric, wavelet or random sigmoid-like functions are commonly used as front ends to linear fits. Also among the nonlinear techniques are artificial neural networks which fit customized nonlinear transformations to the problem.

What constitutes the best fit to a data set? As a regression for any nontrivial will inevitably have errors, one needs to pay attention to which errors are minimized. The most widely used measure of error is squared error. However, methods of estimation that minimize squared error are particularly vulnerable to outliers in a data set. Alternative measures of error are the sum of absolute values of errors, called least absolute deviations (LAD) and the median squared error. These alternatives fall under the heading of robust regression due to their robustness to outliers.

The widely used linear least squares regression technique is arguably used partly because many software packages include it as a ready-made black box routine. The most straightforward form of applying least squares regression seeks to optimize a matrix of coefficients, $\beta$, such that a matrix of inputs, X, where each column corresponds to an input variable (frequently called features by the machine learning community) and each row corresponds to a data sample can best predict the value of outputs, given by a matrix, Y, where each row is an out put sample corresponding the the input samples in $\mathrm{X}$ and each column is an output variable. The equation to be fit, the normal equation, is $X \beta=Y$. The solution to this is $\beta=\left(X^{T} X\right)^{-1} X^{T} Y$.

It is frequently the case, particularly if a fit involves several highly correlated variables, that the matrix $\mathrm{X}$ is very nearly singular. In this case, the normal equation will produce estimates of $\beta$ that fit noise, causing to highly correlated variables to have large coefficients which cancel each other in effect. Two main approaches to counteract this problem. One is two use the techniques of singular value decomposition, the description of which is outside the scope of this dissertation. The other 
is to add a penalty while minimizing error for large coefficients in $\beta$. If the squared coefficients are penalized, we say the L2 norm of $\beta$ is minimized along with error. This is easy to incorporate into solution for the normal equations, $\beta=\left(X^{T} X-\lambda I\right)^{-1} X^{T} Y$, where $\lambda$ is a parameter to be tuned and determines the strength of the penalty.This approach is often called ridge regression. If the absolute values of $\beta$ are penalized, we say the L1 norm of $\beta$ is minimized. L1 penalized minimization is more difficult to implement, but, under the name of LASSO regression, it has the use of performing automatic feature selection. [Friedman et al. (2001)]

\section{iii. Simulated Annealing}

Simulated annealing (SA) is a generalized optimization technique that performs minimization or maximization in a way analogous to and inspired by the annealing of a slowly cooling metal [Press et al. (1992)]. SA operates according to the following sequence, which is iterated until a stopping condition is found. (1) At each iteration of the main loop, $i$, a pseudo-random variation is added to each parameter to be optimized. (2) If the new cost function, $f$, to be minimized is less than the previous value, $\Delta f=f_{i}-f_{i-1} \leq 0$, the new parameter set is accepted. Else, the new parameter set is accepted with probability $p(\Delta f)=\exp (-\Delta f / T)$, where $T$ as a value analogous to temperature. The probability of an uphill move enables SA to escape local minima to find new local minima closer to the global minimum. (3) The parameter $T$ is lowered, analogous to slow cooling. Also, the scale of pseudo-random variations (for continuous variable problems) is lowered, allowing the solution to settle in what is ideally the global minimum. SA is computationally expensive and slow, but it has the advantages of allowing the solution of highly nonlinear optimization problems, including those which involve discontinuities, and of allowing a freedom of choice in the cost function to be minimized (e.g. squared error, absolute error, etc.).

\section{iv. Simulated Evolution}

While SA is inspired by the physical processes of cooling metals, simulated evolution is inspired by the processes of biological evolution. There four main schools of evolutionary computation are genetic algorithms [Goldberg et al. (1989)], genetic programming [Koza (1992)], evolutionary programming [Fogel (1999)], and evolution strategies [Schwefel et al. (1995)]. Evolution strategies have advantages over other evolutionary approached for the kinds of numerical optimization prob- 
lems one often encounters in physics and engineering, where the continuous numeric variables one encounters can be searched with mutations with a gaussian distribution.

In evolution strategies (ES), a parent population of candidate solutions is generated. An offspring population is generated, typically via addition of random values i.e. mutations to the parent solutions, but sometimes also through swapping coefficients between parents i.e. recombination. The best solutions (according to the selection criteria for the problem at hand) among parents and offspring are kept to become parents for the next generation. This continues until either a good enough solution is found or alloted computer resources are exhausted. ES, as with SA, are useful for solving nonlinear problems, problems with discontinuities, and problems with local minima. Because ES are easier to understand, program, and tune to a problem than SA, while producing results roughly on par with other methods, they are deserving of more attention among the research communities that utilize generalized statistical fitting and optimization. [Schwefel et al. (1995)]

\section{Geomagnetic Storms and Substorms}

During periods of time when large amounts of energy are transfered from the solar wind to the magnetosphere, magnetic disturbances known as geomagnetic storms and substorms can occur. The exact relationship between storms and substorms is subject to much debate.

\section{Geomagnetic Storms}

During intervals of relatively strong, southward IMF, a sufficient amount of energy and flux is transferred to the magnetosphere by the magnetic reconnection process for large, global geomagnetic disturbances to occur. These disturbances manifest as a substantial weakening of Earth's surface magnetic field at low and mid latitudes, measured by $D_{S T}$ and other storm-time magnetic depression indices discussed above. They follow a progression beginning with onset, when an impulse of SW pressure often (but not always) causes a brief strengthening of ground magnetic field. Onset is followed by the storm main phase, where the strength of magnetic depression increases. Finally, during recovery phase, a relaxation of storm-time magnetic depression occurs. Because these disturbances are time varying, damaging electric currents can be induced in large, conductive structures such as power grids and oil pipelines, resulting in damage to these structures. Geomag- 
netic storms have also been responsible for the detonation of magnetic mines [Knipp et al. (2018)]. The largest recorded geomagnetic storm occurred in 1859 [Cliver and Svalgaard (2004)]. If another storm comparable to that of 1859 were to occur today, it has been projected that power in some areas could take up to a decade to restore!

Although geomagnetic storms have been extensively studied, there are still ongoing debates concerning which current systems contribute the most to the measured storm-time disturbances.

\section{i. Magnetopause Current Contributions}

Magnetopause currents can be thought of as a region of current separating regions of IMF from regions dominated by Earth's magnetic field. During geomagnetic storms, the SW dynamic pressure often increases, compressing the day-side magnetosphere, intensifying the magnetopause currents, and bringing them closer to Earth. The effect of magnetopause currents is to strengthen the surface magnetic field at low and mid latitudes (see figure 1.18 part a). From the pressure balance considerations, the effect of magnetopause currents is proportional to the square root of SW dynamic (or ram) pressure, $\sqrt{p_{d y n}}$. The exact magnitude of magnetopause caused magnetic disturbances has been calculated by a number of empirical and theoretical studies, with often contradictory results.

Magnetopause effects upon storm-time magnetic depressions are less controversial than other current systems. However, there are some reasons to believe that the magnitude of magnetopause effect evolves during the progression of geomagnetic storms, due to the erosion of the day-side magnetosphere. In this dissertation, we will consider the contribution to be described in the standard, non-time dependent version.

\section{ii. Ring Current Contributions}

Ring currents are composed of trapped particles in the inner magnetosphere. The symmetric ring current closes with itself, encircling the Earth. The partial ring currents are found on the nightside, closing through the ionosphere via the R2 FAC. Both partial and symmetric ring currents contribute to the storm-time magnetic depression of Earth's magnetic field (see figure 1.18 parts b and e).

Since the 1960s, the standard treatment of geomagnetic storms is to regard the storm-time magnetic depression as being primarily due to symmetric ring current. This state of affairs resulted from the development of the Dessler-Parker-Schopke relationship, where the magnetic effect of the 
ring current, $\Delta B_{\text {particles }}$ is related to the energy of trapped particles in the symmetric ring current by $\Delta B_{\text {particles }} / B_{o}=-2 / 3 W_{\text {particles }} \hat{e_{Z}} / W_{\text {mag }}$, where $W_{\text {mag }}$ is the energy in Earth's dipole magnetic field above Earth's surface, $W_{\text {particles }}$ is the energy in trapped ring current particles, and $B_{o}$ is the equatorial surface magnetic field [Kivelson and Russell (1995)]. Accordingly, it is common to see publications where $D_{S T}$ and other proxies for storm-time magnetic depression (STMD), once corrected for magnetopause currents, are treated as a proxy for symmetric ring current.

\section{iii. Cross-Tail Current Contributions}

Another contributer to storm-time magnetic depression is the cross-tail current (see figure 1.18 part c). In the 1990s, Maltsev and his collaborators produced an approximate derivation for the effect of tail currents [Maltsev et al. (1996); Arykov and Maltsev (1996)], showing that tail currents should have a $D_{S T}$ contribution proportional to the amount of magnetic flux in the outer magnetosphere according to $H=F / 2 S \approx\left(2 B_{o} / 9\right) \sin ^{6}(\theta)$, where $F$ is the magnetic flux outside the stable trapping region where ring currents exist, $S$ is the area of the stable trapping region (see figure 1.19), and $\theta$ is the average magnetic colatitude of the equatorward boundary of the Auroral oval. The derivation showed that tail currents could potentially be the dominant contributer to $D_{S T}$. Vasylinas (2006) performed a more rigorous derivation of a generalized Dessler-Parker-Schopke relationship, including the effects of all contributing current systems, in which he also found that tail flux can be related to the contribution of tail currents to $D_{S T}$.

\section{iv. Controversies Associated with Contributions of Specific Current Systems}

A number of studies have attempted to answer the question of how important each current system is to $D_{S T}$. The widely cited study of Greenspan and Hamilton [Greenspan and Hamilton (2000)] claimed verification of the Dessler-Parker-Schopke relationship. However, Greenspan and Hamilton show little correlation between dayside (and thus symmetric) ring current and $D_{S T}$. Thus, the ring current contribution to geomagnetic storms is arguably due to partial, rather than symmetric, ring currents, at least prior to recovery phase. Lopez et al. (2015) analyzed in situ observations from an intense, individual storm showing little ring current contribution for that event. Likewise, Roeder et al. (1996) used measured particle flux to show that ring current for March 1991 storm could account for only 30 to 50 percent of $D_{S T}$. 
Empirical models have also been used to answer the question of relative current contributions. Turner et al. (2002) used the empirically derived Tsyganenko T96 and T89 models to calculate relative contributions tail current to $D_{S T}$ for modeled events. They found a 25 percent contribution from tail currents. Conversely, Alexeev et al. (1996) used a different empirical model to show tail current contributions greater than 50 percent. Deficiencies in the empirical models used render such results less than conclusive. Ganushkina et al. (2004) modified the T89 model and fit the modeled current system contributions to reproduce in situ magnetic measurements from multiple spacecraft. They found that tail contributions dominates early in a storm, whereas ring current dominates as the storm progresses. They also found that weaker storms are more dominated by tail currents whereas stronger storms are more ring current dominated.

\section{Geomagnetic Substorms}

Geomagnetic substorms are processes whereby energy loads into the tail region and then unloads in an explosive release, triggered by instabilities in the tail. The exact nature of the instabilities leading to substorms, and even the very definition of substorms, are subject to much debate. However, after a period of accumulation of energy in the magnetotail (e.g. the growth phase) the explosive release of tail energy (e.g. the expansion phase) results in the near Earth tail becoming more dipole-like, thus being called dipolarizations. As the expansion phase onset occurs, the current sheet in the tail region is partly disrupted, and the electric current is routed to the night-side ionosphere via the substorm current wedge ( see figure 1.20). After expansion phase exhausts itself, a recovery phase occurs where ground magnetic disturbances relax. The auroral zones experience brightenings accompanied with magnetic disturbances, tracked by the AE indices (see figure 1.21). [Kepko et al. (2015)]

\section{Controversies Concerning the Storm-Substorm Relationship}

In this dissertations, substorms are of interest primarily because of their relations to geomagnetic storms. Although storms nearly always are accompanied by substorms, the reverse is not true.

Historically, substorms were named because geomagnetic storms were thought to by built from many substorms clustered closely in time. Today, substorms are thought to play a contributing role in particle injections into the ring current. If this view is correct, and ring current is an major 
contributer to $D_{S T}$, substorms ought to be associated with strengthening of $D_{S T}$.

Substorms are a mechanism for releasing energy and flux from the tail region. In the view that $D_{S T}$ is dominated by tail current contributions, one would expect the $D_{S T}$ to be weakened by substorm activity. Iyemori and Rao [Iyemori and Rao (1996)] performed a high time resolution superposed epoch analysis at high time resolution suggesting that storm-time magnetic depression growth slows when a substorm occurs.

Conversely, substorms are responsible for enhanced ionospheric outflow of oxygen ions [Moore et al. (1999)] resulting in eventual enhancement of the ring current. In situ observations have found that $\mathrm{O}+$ ions peak near the storm maximum [Hamilton et al. (1988)]. Theories of how $\mathrm{O}+$ ions make their way to the ring current include those in which oxygen moves directly into the ring current and those in which the $\mathrm{O}+$ ions first enter the tail lobes, pass to the tail current sheet, and finally enter the ring current [Nosé et al. (2010)]. 


\section{Chapter II}

\section{Rectified Solar Wind Electric Field: Time Scale Effects}

As discussed in chapter 1, a variety of coupling functions have been constructed from solar wind parameters which are intended to model the driving influence of the solar wind upon the Earth's magnetosphere and its geomagnetic activity. Because solar wind coupling functions will be utilized extensively in later chapters, evidence will be presented in this chapter that the rectified solar wind electric field, $v B_{S}$, probably the simplest widely used solar wind coupling function, remains competitive with most sophisticated coupling functions, at least for prediction of geomagnetic storm indices.

In section 2 , we will demonstrate that $v B_{S}$, when time scales are considered, can at least partly account for the functional forms of some of the alternative coupling functions found in the literature and specifically the optimal forms found in section 2 .

In section 3, the injection term from the Burton model and other geomagnetic activity measures will be tested via cross-correlation against candidate coupling functions and it will be seen that it will be shown that $v B_{S}$, already effective for $D_{S T}$ prediction tasks, performs better when various approaches to include time scale dependent effects are utilized.

In section 3, Burton type differential equation models are fit empirically to solar wind and $D_{S T}$ data sets from the NASA Omni Database. It will be shown that, although sophisticated solar wind coupling functions can provide a better fit to the data at the cost of many additional free model 
parameters, $v B_{S}$ exhibits a remarkably good minimization of error.

\section{A. Solar Wind Statistics and Coupling Functions utilizing Hourly Averages in Solar Wind parameters}

Many studies of solar wind coupling (see table 2.1) and ring current injection make use of hourly averages in solar wind parameters [e.g.. Newell et al. (2006); Newell et al. (2007); Newell et al. (2008); Boynton et al. (2011)]. Though Newell et al. (2007) consider that the physically relevant parameters may differ at shorter time scales, citing as an example that dynamic pressure changes may have a more significant effect, they generally regarded the empirically fit in that study to be related to underlying physical processes. Here, however, we argue that statistical effects due to averaging are also relevant. A literature search found that this notion had also been considered, but not fully developed, by Svalgaard (1977).

The most obvious statistical effect to which we refer is most clearly illustrated in figure 2.1. Consider that if $v B_{S}$ is the correct solar wind coupling function, and experience has shown that this is at least approximately true, then the interval shown at the one minute timescale would have nonzero energy transfer to the magnetosphere for part of the interval in question. However, as the average magnetic field is northward for this interval, the formula using hourly average values of SW parameters would report zero coupling and thus zero storm injection. The most commonly encountered alternative functions are "leaky rectifier functions", with an IMF clock angle dependency given as a power of $\sin \left(\Theta_{C} / 2\right)$, where $\Theta_{C}=\arctan \left(B_{Y} / B_{Z}\right)$. Such functions would yield a nonzero coupling for the interval shown, just as with $v B_{S}$ when used with data of higher time resolution.

To study these statistical effects, we downloaded 1 minute solar wind parameters from the spacecraft specific Omni 2 database for the years 1973 through 2015, inclusive. The low resolution hourly values from the Omni 2 database were used for comparison. The spacecraft specific sets were used because we can ensure that the same monitor was used for a given hour in both high and low time resolution sets. We make the following assumptions. 1. The rectified dawn-dusk electric field, $v B_{S}$, is close in form to the correct instantaneous solar wind coupling function. 2. The dawn-dusk electric field applied to the magnetosphere of standard size results in a time varying 
net electromotance. As power consumed by a circuit element with respect to an applied time varying electromotance is correctly calculated using the root-mean-squared (RMS) voltage, the appropriate hourly value of $v B_{S}$ is the RMS value, $\sqrt{<}\left(v B_{S}\right)^{2}>$. We searched though functions of form $n^{\alpha} v^{\beta} B_{T}^{\gamma} \sin \left(\Theta_{C} / 2\right)^{\delta}$, widely used is SW-magnetosphere coupling studies (following Vasyliunas et al. (1982)), to find the one that correlates best to hourly RMS of rectified SW electric field, $\sqrt{<}\left(v B_{S}\right)^{2}>$. For each hour, we used the 1-minute data from the same solar wind monitor as used by the low resolution Omni database for the same hour. The resulting coupling function of the form specified above with maximum correlation to the RMS of $v B_{S}$ is $n^{0.1} v^{1.3} B_{T}^{0.9} \sin \left(\Theta_{C} / 2\right)^{4.4}$. This is significant because it is similar to those coupling functions found in the literature but which were constructed empirically to maximize correlation with magnetospheric state variables, often under the assumption that the specifics may reveal something more fundamental about solar wind magnetosphere coupling.

Many solar wind coupling functions reported to work well have $\alpha \geq 0, \beta \geq 1, \alpha \leq 1,5>\delta \geq$ 2 , which is the qualitative behavior shown to statistically emerge for $v B_{S}$. Thus, the functional forms of solar wind coupling reported in the literature for hourly timescales are likely to be at least partly due to the statistical properties of the solar wind parameters. Note that the emergence of a function nonlinear in $n, v$, and $B_{T}$ is not unreasonable as these parameters are not statistically independent as seen in figure 2.2 [Maltsev and Rezhenov (2003)].

We follow this result by testing the correlation between measures of geomagnetic activity and SWCFs. Following in the footsteps of Garrett et al. (1974) and some investigators referenced in Feldstein (1992), we construct a version of $v B_{S}$ supplemented by $\sigma_{B}$, which is the hourly vector standard deviation in IMF as reported in the Omni 2 database. The scalar and z component standard deviations of were also tested, but found to produce coupling functions with lower correlations to magnetospheric state variables. The results from functions $v\left(B_{S}+0.5 \sigma_{B}\right)$ and $v\left(B_{S}+0.67 \sigma_{B}\right)$ are reported along with the universal coupling function proposed by Newell et al. (2007), the formula of Wygant et al. [1983], and $v B_{S}$ using the hourly values from Omni 2 as well as the RMS and mean values of 1-minute $v B_{S}$. The cross-correlations between the SWCFs considered is shown in table 2.2. It can be seen that simple functions which attempt to introduce SW variability by introducing $\sigma_{B}$ generally correlate better to Newell's more complicated "universal coupling function" than the simple hourly $v B_{S}$. 


\section{B. How Solar Wind Coupling Functions Using Hourly Solar Wind Variability Compare with Other Widely Used Functions}

Following in the footsteps of Garrett [1974] and some investigators referenced in Feldstein [1992], we construct a version of $v B_{S}$ supplemented by $\sigma_{B}$, the hourly vector standard deviation in IMF as reported in the Omni 2 database. The scalar and z component standard deviations of were also tested, but found to produce coupling functions with lower correlations to magnetospheric state variables.

The functions $v\left(B_{S}+0.5 \sigma B\right)$ and $v\left(B_{S}+0.67 \sigma B\right)$ were tested along with the universal coupling function proposed by Newell et al. [2007], the formula of Wygant et al. [1983], and $v B_{S}$ using the hourly values from Omni 2 as well as the RMS and mean values of 1-minute $v B_{S}$. The results are summarized in tables 2.1 and 2.2 .

The geomagnetic activity measures were processed as follows. For the Auroral Electrojet indices, we used the weighted averaging scheme specified in Newell et. Al [2007], shown in figure 2.3. For the $D_{S T}$ index, we correlated the injection calculated from the model of Burton et al. [1975] with the SWCFs weighted with weights, determined by trial and error for best correlation, of 0.75 and 0.25 for the previous and simultaneous hours, respectively. For PC north index, we used the simultaneous SWCF in our correlations.

For the results shown in table 2.4, in general, the RMS average of $v B_{S}$ derived from one minute solar wind data outperformed the simple mean of one minute $v B_{S}$, which itself outperformed $v B_{S}$ calculated from 1-hour averages of solar wind parameters. The functions $v\left(B_{S}+0.5 \sigma B\right)$ and $v\left(B_{S}+0.67 \sigma B\right)$ performed favorably in comparison to the aforementioned SWCFs. It is also notable that Wygant's coupling function, $E_{W A V}$, though it has a reputation for performing well, did not predict the selected measures of magnetospheric state better than any function taking solar wind variability into account. Only $d \Phi_{M P} / d t$ had higher correlations for most magnetospheric state variables, excepting the $D_{S T}$ injection, which favored the SW variability based functions. 


\section{Burton Type Differential Equation Models of Geomagnetic Storms}

A family of models with a long history of successful use are what we will refer to in this dissertation as Burton models, as exemplified by Burton et al. (1975). A number of models in this family have been constructed, as can be seen in Feldstein (1992) and references therein. Burton models are 1st order differential equation models with an injection term and a decay term determining the rate of change of storm-time magnetic depression. It can be expressed as $\dot{D} *=Q-D * / \tau$, where $D *$ is the storm-time magnetic depression with magnetopause current effects removed according to $D *=D-b \sqrt{P}+c$, where $\mathrm{b}$ and $\mathrm{c}$ are constants determined from theory or empirical fitting. $Q$ is the injection fuction, typically given as a SW coupling function to drive magnetospheric activity, so named because it is traditionally thought to relate to ring current particle injections. $\tau$ is a decay constant determining the relaxation rate of storm disturbance.

A number of attempts have been made to enhance the Burton model. Most notable are attempts to allow $\tau$ to vary according to SW parameter [O'Brien and Mcpherron; Maltsev and Rezhenov (2003)] or according to storm phase [Vassiliadis et al. (1999)]. However, for our purposes of studying SW coupling, we restrict ourselves to the constant $\tau$ case.

\section{Optimizing the Burton Model and its Injection Function}

The entire hourly Omni 2 database of solar wind parameters and $D_{S T}$ from 1963 through April 2016 was downloaded from http://omniweb.gsfc.nasa.gov. This provides us nearly 300,000 hours of data for creating statistical fits. As with O'Brien and McPherron, we assume that the lag time between solar wind driving and ring current injection permits us to fit the Burton equation using forward differences according to the equation: $D_{S T}^{*}(t+1)-D_{S T}^{*}(t)=Q(t)-D_{S T}^{*}(t) / \tau$.

Due to discontinuities of this equation with of some forms of $\mathrm{Q}$, linear least squares fitting techniques are of limited utility. Accordingly, we adopt simulated annealing as a nonlinear fitting technique [Press et al. (1992); Corana et al. (1987)]. Simulated annealing has the further advantage that it can be easily used to minimize the sum of absolute error to produce a least absolute deviations fit as easily as it can minimize the sum of squared error. Although minimization of squared error is the most commonly used statistical fitting technique, it is sensitive to statistical outliers. Sensitivity 
to outliers is not necessarily a bad thing if those outliers happen to be the the disturbed intervals that are are of greatest interest. Nonetheless, minimization of absolute error is known to be more robust to outliers (Dielman (1986)) and is done to check the consistency of fitted model parameters. As a further check on consistency of our results, each simulated annealing fit was repeated using the even year and odd year subsets.

We began by performing a fit the the injection function chosen by Burton et al. (1975), $Q=$ $\min \left(\alpha\left(v B_{S}-E_{C}\right), 0\right)$. This established a basis of comparison with other candidate functions. Preliminary results showed that $E_{C}$ was small with values scattered between 0 and $0.13 \mathrm{mV} / \mathrm{m}$. Consequently, we set $E_{C}$ to 0 with negligible effect on residual error. We then replace $v B_{S}$ with a function of form $n^{\alpha} v^{\beta} B_{T}^{\gamma} \sin \left(\Theta_{C} / 2\right)^{\delta}$, to find the best fits. Our results are summarized in table 2.4. In each case we find that clock angle dependence parameter $5>\delta>4.3$. We note that this matches most closely the clock angle dependence and exponent values found by Milan et al. [2012] Milan et al. (2012), with the exception that be found a small but nonzero dependence on solar wind density. This is not unsurprising as several investigators have found that the solar wind particle density has an influence on storm development (e.g. see Weigel (2010)).

In the previous section, we showed that a simple SWCF such $v B_{S}$ can statistically result in functional dependencies similar to those seen. This poses a problem for any who attempt to learn about solar wind magnetosphere interactions using hourly averaged data. In order to minimize this problem, we again use simulated annealing with the same data sets as above to simultaneously determine the parameters of a Burton type model and a SWCF of form $n^{\alpha} v^{\beta} B_{T}^{\gamma} \sin \left(\Theta_{C} / 2\right)^{\delta}$, but this time using only those intervals where vector standard deviation in IMF, $\sigma B$, as reported by Omni is less that half the magnitude of tangential magnetic field. Our results are summarized in table 2.4. In general, the clock angle dependence, when only periods of low relative SW variability are considered, became more selective i.e. closer to the half wave rectifier. It can also be seen that the exponents for density and speed became closer to zero and unity, respectively, supporting our hypothesis that nontrivial statistical dependencies between solar wind parameters are partly responsible for empirically derived SWCFs with parameters raised to exponents differing

We also used the linear combination of $B_{S}$ and vector standard deviation in IMF, in a series of simulated annealing fits to see how such an approach can minimize residual error. The results are summarized at the bottom of table 2.5. One can see that residual error is not significantly 
worse that that found using functions of the form $n^{\alpha} v^{\beta} B_{T}^{\gamma} \sin \left(\Theta_{C} / 2\right)^{\delta}$, which have many more free parameters to optimize. This strongly suggests that the half wave rectifier, $v B_{S}$, is very close to the true driver of $D_{S T}$.

\section{Explanation}

Although figure 2.1 should make it clear why the apparent clock angle dependence would differ from the half wave rectifier function when SW data from long time scales is used, it is less intuitive that SW coupling functions nonlinear with respect to density, flow speed, and magnetic field would statistically emerge. In figure 2.2, we see that Maltsev and Rezhenov (2003) showed that SW parameters are not independent. Moreover, several SW parameters do not follow Gaussian distributions Veselovsky et al. (2010). We believe that the lack of statistical independence between SW parameters combined with the non-Gaussian distributions which violate the assumptions of linear correlation methods combine to produce the observed nonlinearities.

There are good reasons to ask why $v B_{S}$ seems to perform so well. The success of $v B_{S}$ in so many SW-magnetosphere coupling studies, plus the fact that we have show $v B_{S}$ can account for at least some of the successes of more complicated coupling functions, seems to imply that the magnetosphere receives much of its energy from the SW via anti-parallel merging. The main stream view regards component reconnection, rather than anti-parallel reconnection, as the primary mode of reconnection. We offer two possible answers to this objection. One possibility is that antiparallel reconnection really is the dominant mode of merging in the magnetosphere. Evidence for this possibility can be found from both simulation studies (Russell et al. (2003); Park et al.) and from observations (Coleman et al.;Fuselier et al. (2010)). An alternate possibility is that their exists a critical clock angle (Sonnerup (1974)) for component reconnection. In the critical angle paradigm, IMF orientations Northward of the critical angle would not lead to merging at the magnetopause, thus leading to a clock angle dependence similar to the half wave rectifier model. In Borovsky (2013), the critical angle was considered but rejected partly on the grounds that its use does not lead to improved correlation between the coupling function considered and geomagnetic indices. However, Borovsky made use of hourly averaged SW data to test the predictive power of his functions. A better test of the critical angle hypothesis would utilize higher time-resolution 
data.

\section{E. Conclusion}

The primary conclusion of this chapter is that complicated SW coupling functions for the magnetosphere do not necessarily represent the true response of magnetospheric behavior to solar wind driving, particularly if the functions under consideration are empirically derived and/or validated using data averaged over a longer timescale. The practical considerations are that experimental studies should take into account the time scales over which the solar wind parameters vary. Although some (mostly older) studies have considered SW parameter variations (e.g.. Pisarskij et al. (1989)), the larger geospace research community has not taken these issues into consideration, leading to potentially misleading results. We believe this is partly because no specific study has been widely disseminated to warn of this problem, and partly because taking countermeasures to this issue adds extra complication to an empirical investigation. Our recommendations, which are reflected in subsequent chapters of this dissertation, are to do the following:

1. When it is necessary to use hourly averaged data in an empirical study to reveal the functional dependence of magnetospheric behavior upon SW parameters, one approach to better results is to exclude intervals when IMF variations are large compared to IMF magnitude or the magnitude of $\operatorname{IMF} B_{Z}$.

2. If predicting the behavior of the magnetosphere is the goal, including a function of IMF variability as a predictor variable can improve the results from hourly averaged SW data.

3. When shorter timescale data is available, the use of averaged (or RMS averaged) coupling functions can help avoid the aforementioned pitfalls.

4. If one seeks to perform an empirical study at a faster time cadence, one should consider that errors in propagation of solar wind data to the magnetosphere from the SW monitor can be considerable, thus leading to similar statistical errors that emerge from uncritically using hourly averaged SW data. 


\section{Chapter III}

\section{Impulse Response and The}

\section{Storm-Substorm Relationship}

Impulse response functions (IRFs) are useful for determining the time dependent development of an index with respect to a proposed driver. They were first used by Iyemori et al. (1979) to study the response of $D_{S T}$ and auroral electrojet indices with respect to IMF $B_{S}$ and $v B_{S}$. They noted a second development in the response of $D_{S T}$, an increase in impulse response several hours after the driving impulse had happened appearing as a bump on the plotted IRF. They interpreted this as evidence of an oscillatory behavior.

Weigel (2010) also did an extensive study where he generated IRFs after binning data from a series of geomagnetic storms by solar wind density at the beginning of the events. He found the best fits when simply using $v B_{S}$ as a driver and that $v B_{S}$ is more geoeffective in driving storms when the solar wind density is higher than average. He attributed this to the solar wind ions being a source of ring current particle, but admits another possibility. In paragraph 56, he states, An alternative explanation of the change in geoefficiency is that under the lowdensity solar wind conditions, ring current energy is diverted into another magnetospheric system, such as the auroral electrojets. Although Weigel did not comment on the second development, it was clearly visible and more prominent in the IRFs produced from low solar wind density events. In this chapter, we look for evidence in favor of that alternate explanation and seek to better understand the cause of the second development in $D_{S T}$ IRF. 
In section A, we explain the general methodology taken for IRF analysis in this chapter.

In section $\mathrm{B}$, we use IRF analysis to establish that $v B_{S}$ is adequate as a solar wind driver and that there in no need to include dependencies of IMF $B_{Y}$.

In section C, we use IRF analysis to support Weigel's alternative hypothesis that energy is diverted from the systems contributing to the $D_{S T}$ index and into auroral electrojets.

In section $\mathrm{D}$, we turn our attention to the second development in $D_{S T}$ IRF. Based on the evidence, we argue that this can be explained by contributions of two different current systems to the storm-time magnetic depression indices. As the relative contributions of ring currents versus tail currents have been a source of ongoing controversy, this gives further credence to the notion that tail currents are more significant than often thought in the storm-time magnetic depression.

In section $\mathrm{E}$, the impulse response of magnetic depression in four local time sectors is evaluated using the APL SMR sector based indices. The process of ring current development and symmetrization is observed through its IRF. It is shown that substorm dependence in the midnight sector is quite different from other sectors. Evidence for oscillatory behavior is seen in the midnight and dawn sectors only.

\section{A. Impulse Response Function Methodology}

The results in this chapter are established by performing fits of the IRF of geomagnetic indices to various drivers. The IRF function with respect to a single driver variable can, in general, be written as $W(t)=\int_{0}^{\infty} h(\tau) f(t-\tau) d x$, where $W(t)$ is the output variable, $\tau$ is the lag time, $f(t-\tau$ is the input variable, and $h(\tau)$ is the impulse response. As we are fitting IRFs of discrete time series where more than one driver variable is considered, this becomes $W(t)=\sum_{a} \sum_{i} h_{a}\left(\tau_{i}\right) f_{a}\left(t-\tau_{i}\right)$, where $a$ runs over driver the set of driver variables and $i$ runs over the (finite) set of lags considered.

To obtain our IRFs, we use regression techniques to fit $h_{a}\left(\tau_{i}\right)$ to data. We use linear least squares fits as the primary fitting technique. However, several fits were repeated using the more computationally expensive two-membered evolution strategies to obtain least absolute deviation fits. No noticeable difference was discerned between the results of the two fitting techniques, so we consequently focused our efforts on least squares fits. We established error bars for IRF coefficients by producing 50 randomly selected, 50 percent subsets. The error bars reported were median 
absolute deviations from the median values of the impulse response coefficients.

For several IRF fits, it was desired to produce IRFs from binned subsets. In Weigel (2010), the binning was done according to starting values of the discriminant variable to produce independent IRF fits. However, we separated the driver variables into multiple sets, thresholded according to the discriminant variable. This permits us to deal consistently with situations where the discriminant variable (typically density) varies during an event.

\section{B. Evaluation of The Solar Wind Dependence of $D_{S T}$}

As a preliminary measure, we first use hourly data to generate IRFs for $D_{S T}$ to determine if $v B_{S}$ is truly sufficient for this study. All available data from the low resolution (1-hour) Omni Database, from November 1963 through to January 2017, was used to compute these IRFs. These results are shown in figures 3.1-3.4. In figure 3.1, it the impulse response of $D_{S T}$ with respect to $v B_{S}$ is shown. It can be seen that the effect of $v B_{S}$ upon $D_{S T}$ is close to what one would expect, with a slowly decaying response. The error bars for the coefficients are small relative to the coefficients themselves. In figure 3.2, the IRF of $D_{S T}$ with respect to the square root of dynamic pressure is shown. This shows that only dynamic pressures roughly concurrent with a $D_{S T}$ reading significantly affect the $D_{S T}$ index. The magnitude of the largest coefficient is consistent with the pressure (magnetopause current) corrections found in most of the literature [add citations]. In figure 3.3, it can be seen that the impulse response coefficients showing the influence of IMF $v B_{Y}$ magnitude are dwarfed by the both those of $v B_{S}$ and by their own error bars. In figure 3.4, standard deviation of interplanetary electric field, $v \sigma_{B}$, derived from standard deviation of vector IMF, $\sigma_{B}$, has a much stronger and more consistent effect (evidenced by smaller relative error bars) on $D_{S T}$ than IMF By. As this shows effects not modeled with hourly averages, as argued from solar wind statistics in chapter 2, we choose to continue with the higher time resolution $\mathrm{Sym} / \mathrm{H}$ and $\mathrm{SMR}$ indices in place of $D_{S T}$ and high time resolution solar wind parameters from the Omni data base. 


\section{A Comparison of Sym/H and AL Impulse Response under High and Low SW Density Conditions}

Weigel's alternative hypothesis was that weaker solar wind driving of $D_{S T}$ during intervals with low solar wind density could be due to energy being diverted into the aurorae. Since substorm auroral electrojets get their energy from the magnetotail, which is thought by some [Maltsev (2004)] to dominate storm-time magnetic depression, this would, if demonstrated, be evidence in favor of a greater tail current contribution to $D_{S T}$ and $\mathrm{Sym} / \mathrm{H}$. We investigate this by generating IRFs for both the AL substorm index and Sym/H. These IRFs are binned according to concurrent solar wind density, where the median value of density was chosen as the boundary between bins. It should be pointed out that our approach to binning by density was different from that of Weigel. Whereas Weigel attempted to bin by density at the beginning of an event, we made 2 sets of $v B_{S}$ data, thresholded by SW density, which were don't fed into the regression and used for the entire data set. The results are shown in figures 3.5-3.7. Comparing figures 3.5 and 3.6, one can observe that high density solar wind is significantly (i.e. relative to the errors in the coefficients) more geoeffective for driving Sym/H, as demonstrated by larger peak IRF coefficients. Conversely, figures 3.8 and 3.9 show that $v B_{S}$ during intervals with low solar wind density is more geoeffective for driving AL than during high density intervals. One can see that Weigel's alternative explanation is consistent with the result that $v B_{S}$ is a more geoeffective driver of the auroral AL index for low solar wind densities. More energy is likely diverted from the tail region into the auroral current systems during low density intervals. Accordingly, this strengthens the position arguing for a greater cross-tail current contribution to $D_{S T}$ than in the standard, ring current centric model.

Also note that the second development in the $D_{S T}$ IRF is present more prominently in the low solar wind density case. Some investigations have found that the main phase disturbance is dominated by tail current contributions, whereas the recovery phase become ring current contributions (e.g. Ohtani et al. (2001); Ganushkina et al. (2004)). Vasyliunas argued that the decay of tail current contribution is offset by transfer of tail energy into the ring current. As tail currents are partly diverted during substorm onsets, Sym/H loses some of its strength. We speculate that oxygen ions, liberated from the ionosphere during substorms, may populate the ring current after a delay of a few hours make to effectively rebuild the storm-time disturbance. Indeed, recent sim- 
ulation results show that the majority of ring current $\mathrm{O}+$ ions are sourced from substorms in the ionosphere [Nakayama et al. (2017)], and observations from the AMPTE/CCE instrument reveal that O+ ions increase after a substorm [Daglis et al. (1999)]. More recent observations using energetic neutral atom (ENA) imaging also show that $\mathrm{O}+$ ions increase after a substorm [Ohtani et al. (2005)]. This explanation is rendered more plausible by a recent study by Michelis et al. [2011] whereby information theory is used to show that information flows from AL to Sym/H. Moon et al. (2006) also found that AL influences $D_{S T}$ after a short time delay. In the next section, we will test our hypothesis.

\section{Substorm Activity, Tail Currents and Sym/H Impulse Re- sponse}

To further test the hypothesis regarding tail current makes an important contribution to storm-time disturbance, we perform an impulse response analysis with respect to $v B_{S}$ only, since ram pressure controlled magnetopause current should have little effect on the auroral boundary (see figures 3.10 and 3.11), for the storm-time magnetic depression one would expect from the formula, $H(A B I)=$ $-8 / 27 B_{e} \sin (\theta)^{6}$, where $B_{e}$, the strength of Earth's dipole magnetic field at the equator, is taken as $31,000 \mathrm{nT}$, and $\theta$ is the radius of the auroral oval in degrees and taken to be $85^{\circ}$ - $\mathrm{ABI}$, given by Maltsev (2004). Some testing (not shown) was also performed using the newer magnetotail (MT) index, which uses ion precipitation data rather that the electron precipitation data used to construct ABI. The results are nearly identical and an inspection of DMSP satellite boundary crossings used to construct these indices, it was seen that the electron and ion boundaries from which ABI and MT are produced nearly always coincide, thus rendering such results redundant. As the equatorward boundary of the auroral oval is strongly related to the state of the magnetotail and its contribution, we look for a similar time dependence with respect to $v B_{S}$ during the early portion of its IRF. Though the IRF produced is noisy and required smoothing, we see a similar fast decay in IRF coefficients, close to that seen of the early portion of decay in Sym/H. We see a stabilization of $\mathrm{H}(\mathrm{ABI})$ decay after a lag of 5 hours, with no second development and lower absolute levels of IRF coefficients. This we interpret as a magnetotail stabilizing or enhancing effect due to the delayed ring current development. This enhancement of the magnetotail by ring current 
was predicted Schulz (1997). It seems that cross-tail currents are indeed responsible for much of the early development of storm-time magnetic depression. As an additional check, we produced another series of IRFs of Sym/H with respect to $v B_{S}, \sqrt{P}$, and the concurrent $\mathrm{H}(\mathrm{ABI})$. Due to the noise present in $\mathrm{ABI}$, we expect the least squares regression method used to produce IRF results to under-represent the contribution of tail currents. The coefficient for $\mathrm{Sym} / \mathrm{H}$ as a function of $\mathrm{H}(\mathrm{ABI})$ is $0.218+/-0.003$, suggesting a lower limit of $20 \%$ contribution to of the tail current system to $\mathrm{Sym} / \mathrm{H}$.

We also generate IRFs that include the AL index as an input variable (see figures 3.12-3.14). Consistent with the hypothesis that the second development is due to a time delayed injection of auroral sourced ions into the ring current, we see that the second development is greatly diminished, replaced with a nontrivial IRF of Sym/H from AL with a time lag somewhat less than that of the 2nd development (perhaps because substorms occur after energy accumulates in response to $v B_{S}$ in the tail for some time) as was seen in the aforementioned second development. However, the error bars for the IRF from AL are relatively large, implying that $\mathrm{AL}$ is a poor proxy for the process causing the second development.

We make the assumption that explosive substorm unloadings from the tail result in much oxygen ion emission into the ring current system. We accordingly produce IRFs for $\mathrm{Sym} / \mathrm{H}$ which include the negative change on $\mathrm{AL}, \delta(A L)$-, as a driving parameter (see figures figure 3.15-3.16). It is seen that the IRF of $\mathrm{Sym} / \mathrm{H}$ with respect to $\delta(A L)$ - has much smaller relative error bars in IRF coefficients, and greatly reduce the second development in IRF from $v B_{S}$ while continuing to coincide with the lag times of the second development, indicating perhaps that ions liberated by explosive substorms are the primary cause of the second development, leading to delayed ring current injection. One can also observe that the response in figure 3.16 shows that the unloading process initially weakens the storm-time disturbance, consistent with the notion that substorms cause an immediate weakening of tail contributions to the disturbance, and also consistent with the results of Iyemori and Rao (1996). However, after a few (approximately 4) hours, the response is to strengthen the disturbance. This is consistent with what one would expect if a lingering ring current is produced by substorm activity, as we are asserting. 


\section{E. Sector SMR Index Impulse Responses}

As seen in figure 1.13 of the introduction chapter, the effects of tail currents should be strongest on the night side. The Supermag project has recently begun providing a storm-time disturbance index, SMR, in which the contributions of Midnight, Dawn, Noon, and Dusk sector disturbances are available as SMR00, SMR06, SMR12, and SMR18 indices respectively. Using SMR indices averaged to 10 minute cadence for the years 1995 through 2016, IRFs were produced with respect to $v B_{S}$ and $\sqrt{P}$ as in the previous sections the results for $v B_{S}$ are shown in figures 3.17 through 3.25. Notable, the sector response is more complex than that of the average index. In particular, the responses in the Midnight and Dawn sectors shows two prominent peaks, with its second development stronger than its first development coming with a lag time of 2 hours. In the Dawn sector, also see a prominent second development with a lag time of 4 hours. In the Noon sector, we observe the second development is more diffuse, but the lag time is approximately 5 hours. In the Dusk sector, the second development is muted, observed as halt in decay beginning around 4 hours and continuing until approximately 7 hours lag time.

We interpret these results as follows. 1. The tail current is cause of the first development with a lag time of 40 to 50 minutes. 2. As energy is withdrawn from the tail through substorm unloading and corresponding the first development decays, $\mathrm{O}+$ ions are produced from the ionosphere, which first make their effects shown on the night side as the partial ring current is energized. 3. The partial ring current begins to symmetrize, causing the second developments to peak later on the day side.

As substorm unloading processes play an important role in the above interpretation, we again produced IRF (see figures 3.26 - 3.34) with the negative dAL/dt as an additional driver of the indices in question. The IRFs of $v B_{S}$, shown in figures again show that second developments are less prominent, although not completely eliminated. The response to negative $\mathrm{dAL} / \mathrm{dt}$ shows interesting behavior. In the midnight and sector, we see relatively large negative coefficients for short lag times. Since storm-time disturbance and negative changes in AL are both negative, this represents a weakening of storm-time disturbance shortly after substorm unloading. At the noon sector, we see a sharp reenforcement of disturbance levels with short lag time which rapidly drops off. However, the noon sector response to substorm activity builds again after 5 hours of lag. 
The dawn and dusk sector response to substorm unloading don't provide as simple a behavioral interpretation, possibly because day and night side behaviors are mixed in these zones.

\section{F. F. Discussion and Conclusions}

In this chapter, we can gathered evidence that the solar wind driving of geomagnetic storm activity (e.g $D_{S T}$, Sym/H activity) is seemingly more geoeffective during high solar wind density intervals because less energy is diverted to the auroral zone during these intervals. Since auroral activity (e.g. substorm activity) is caused by explosive release of energy from the magnetotail, we have interpreted this as evidence consistent with the controversial position that cross-tail currents are an important contributer to storm-time magnetic depression.

We noted that the second development in the IRF for Sym/H from $v B_{S}$ is smaller in those cases where AL is more weakly driven by $v B_{S}$. We interpreted the second development as the signature of ring current injection from oxygen ions from the auroral zone. Thus, the ring current becomes more significant in the later stages of a geomagnetic storm, a result supported by Ganushkina et al. (2004).

We tested our hypothesis by fitting IRFs which included proxies for substorm activity in the auroral zone. These proxies, themselves driven by $v B_{S}$, replaced much of the second development, which was our expectation if the hypothesis was correct.

In finding IRFs for the sector indices, we found the midnight and dawn sectors show particularly strongly defined dual peaks. We draw attention to figure 3.35, taken from a previous IRF study [Vassiliadis et al. (1999)] of $D_{S T}$ response to $v B_{S}$, where the data set was binned according to storm phase and strength. We note that the oscillatory behavior seen for late commencement phase of sting geomagnetic storms shows a similar IRF. Perhaps, the midnight and dawn sector response seen in the present study plays a key role in the behavior detected by Vassiliadis et al. (1999).

These results lend credence to the notion that cross tail currents are more significant than commonly thought as sources of storm-time magnetic depression. Prior to the derivation of Dessler-Parker-Schopke relationship for symmetric ring current, storm-time magnetic depression was thought to be the effect of cross-tail currents. In recent years, the lack of strongly $D_{S T}$ correlated day-side ring current from in-situ measurements have called the standard picture (see the 
schematic in figure 3.36) into question. A more balanced model that includes the effects of both ring current and cross-tail currents is increasingly acknowledged as being necessary (see schematic in figure 3.37). We have further contributed to the understanding of this more balanced view by showing that ring current likely results from delayed substorm sourced ion injections (see schematic in figure 3.38).

Note that the more delayed IRF coefficients show a qualitatively slower decay. This is probably an indication of different current systems with their own, respective, decay rates contributing to $D_{S T}$. Such has been noted in the past (McPherron [1995]), and was explained by McPherron as likely being due to different ion populations in the ring current. Conversely, Feldstein et al. (2000) suggested the interpretation of multiple time-constant decays representing tail and ring current contributions. As reported by Detman and Vassiliadis (1997), past models using a dual decay constants have been shown more successful at $D_{S T}$ prediction that single decay constant models. In particular, Detman and Vassiliadis found decay constants of 3 hours and 24 hours. Similar decay constants of 2 hours and 21 hours were found in Murayama (1986). Our results support the interpretation of Feldstein.

As an example of how the system represented by the schematic in figure 3.38 can be reflected in an IRF, we present figure 3.39. In figure 3.38, an exponential decay with a 2 hour decay constant, representing tail current contributions in our model, is summed with a model ring current contribution. The ring current receives a time delayed injection, driven by tail current decay, spread out (to represent diffusion processes) from 3 hours to 4.5 hours, and decaying with a time constant of 17.3 hours, a typical $\mathrm{O}+$ ion charge exchange decay rate for ring current presented in Hamilton et al. (1988). The model plots show the ring current scaled by factors ranging from 0.1 to 0.4 in increments of 0.05 . Compare figure 3.39 to figure 3.17 and note that lines corresponding to factors of 0.3-0.35 model this IRF well. Compare figure 3.39 to figure 3.11 and note that the model line representing a factor of 0.2 is visually similar to the tail current contribution, perhaps showing the enhancing effect that ring current should have on the magnetotail [Schulz (1997)].

If we are correct by interpreting that the first development of IRF primarily represents tail current contributions and the second development represents $\mathrm{O}+$ ring current signature, this provides important insight into the development of geomagnetic storms. Although it has been known that substorms have competing impacts on tail and ring current contributions to to storm-time depres- 
sion [Daglis et al. (2000); Daglis et al. (1999)], the delayed effectiveness of ring current seems to be original. For example, Ohtani et al. (2005) shows from Energetic Neutral Atom (ENA) imaging that $\mathrm{O}+$ ion content increases in the hour after substorm initiation. In this study, the magnetic effect of such seems delayed a few hours. Either the $\mathrm{O}+$ ring current observed is not fully effective in producing ground magnetic perturbations, or there are subsequent injections. If the former, it could be because the Dessler-Parker-Schopke relation doesn't rigorously apply to all ions, but only stably trapped ions. If the latter, it could be because a second, delayed, more effective, injection occurs, possibly through the indirect path from ionosphere to tail lobe to plasma sheet to ring current as described in Lui et al. (1986).

Our conclusions are the following.

1. The enhancement effect of high SW density on driving of storm-time magnetic depression is due to reduced unloading of energy from the magnetotail. 2. The magnetotail is indeed a significant contributer to the early phase of storm-time magnetic depression, and IRF plots of $\mathrm{H}(\mathrm{ABI})$ suggest it may provide the majority of early storm development. Using ABI index as a regressor variable, we establish a lower limit of $20 \%$, although we suspect the excessing noise in ABI would cause an error minimizing scheme to find a contribution much lower than in fact, especially when $v B_{S}$ also drives the evolution of the auroral oval. 3. After substorm activity removes energy from the magnetotail, the partial ring current grows, probably from ionospheric O+ ions, 2 - 5 hours after SW energy flows into the magnetosphere. The ring current becomes progressively more symmetric from 4 - 7 hours after the SW energy entered the magnetosphere. 4. The auroral boundaries themselves slow their decay around the same time as the ring current develops, confirming the stabilizing effect of ring current upon the magnetotail as predicted by Schulz. 


\section{Chapter IV}

\section{Evidence of Tail Current}

\section{Contributions to Magnetic Storms}

In this chapter, we will argue that storm-time magnetic depression is more strongly related to the equatorward auroral boundaries that is typically thought. The Auroral boundaries will be tracked via Auroral Boundary Index (ABI) and Region 2 field aligned currents (FAC). According to Maltsev and his collaborators, the equatorward boundary of the auroral oval, corresponding to the boundary between inner and outer magnetosphere, is strongly related to the tail contribution to storm-time magnetic depressions.

ABI is generated from Defense Meteorological Satellite Program (DMSP) measurements of electron precipitation. Since ABI is derived from the crossing of a single satellite over one point of the auroral oval, and because the instruments occasionally record unrealistic boundary locations, $\mathrm{ABI}$ is more subject to noise than other indices of magnetospheric behavior. However, ABI has the advantage of tracking an important property relating to the global state of the magnetosphere.

FAC data collected from the AMPERE project is used as a proxy for the Auroral zone. Region 2 FAC patterns are known to coincide with equatorward Auroral zone boundaries, making high cadence maps of FAC a useful tool in the study of geomagnetic storms. According to Maltsev and his collaborators, the equatorward boundary of the auroral oval, corresponding to the boundary between inner and outer magnetosphere, is strongly related to the tail contribution to storm-time magnetic depressions. Past studies of auroral oval behavior were performed using Auroral Boundary 
Index (ABI) and the closely related Magnetotail Index (MT), derived from point boundary crossings of DMSP satellites. ABI and MT are noisy datasets with a number of implausible spikes, rendering them less than ideal monitors of the auroral oval boundaries. Conversely, AMPERE provides a 2 dimensional map of ionospheric FAC, allowing one to observe the entire oval.

\section{A. Auroral Boundary Index}

ABI is generated from Defense Meteorological Satellite Program (DMSP) measurements of electron precipitation. Since ABI is derived from the crossing of a single satellite over one point of the auroral oval, and because the instruments occasionally record unrealistic boundary locations, ABI is more subject to noise than other indices of magnetospheric behavior. However, ABI has the advantage of tracking an important property relating to the global state of the magnetosphere.

In the past, ABI has been regarded as a higher time cadence equivalent of Kp. In fact, the team that releases $\mathrm{ABI}$ also provides a predicted $\mathrm{Kp}$ along with a flag corresponding to how reliable a given prediction will be. The close relation between ABI and Kp has been used to argue that the usefulness of Kp is due to its use as a proxy for Auroral latitude Thomsen (2004). For instance, figure 4.1 (from Feldstein and Starkov (1967)) shows average Auroral boundaries, determined from all-sky cameras, as a function of Kp. In figure 4.2 (from Thomsen (2004)), we see the relationship between ABI and Kp for the year 1991. In this section, we will show that storm-time magnetic depression indices, when scaled logarithmically, are competitive with Kp as proxies for the Auroral boundaries.

The Kp index is derived from K-indices from a number of magnetometers located outside the auroral zone. The K-indices represent deviations from the baseline readings taken during undisturbed intervals during the same 3 hour period of the day. K-indices are quasi-logarithmic, e.g. a $\mathrm{K}$ value of 2 represents a disturbance roughly 1.8 times that measure by a $\mathrm{K}$ value of 1 , et cetera. A linear scaling of $\mathrm{Kp}$ is available as the ap index. Other K-family indices are available. Notably, the am index is a continuous equivalent of ap derived from a network of magnetic observatories more evenly distributed in longitude, thus providing a higher quality index. Attempts have also been made to improve the spatial resolution of am with midnight, dawn, noon, and dusk sector indices and temporal resolution with the 15 minute $\alpha$-15 index. 
In this section, we use data from the years 2000 to 2009, inclusive, on the basis that the entire public data set for $\alpha-15$ index is available for only those years. ABI was obtained from https://cedarweb.vsp.ucar.edu/wiki/index.php/DMSP:ssj4_midnit. ABI values are timestamped with the time of boundary crossing. In the following subsections, all plots were created by assigning the concurrent value of the index we wish to compare to each ABI value. For statistical robustness, we use medians instead of means and use mean deviations from the median for error bars.

\section{K-Family indices versus ABI}

As we aim to show the extent to which storm-time magnetic depression indices are a reasonable proxy for the equatorward Auroral boundary, we first show how well K-family indices correspond to the same. In figure 4.3, we show ap on a true (rather than quasi) logarithmic scale. We see that ap generally corresponds well to ABI, except at extremely disturbed times. Figure 4.4 shows that am is also closely related to ABI. Figures 4.5 through 4.8 establish that the relation between K-family indices and ABI is not restricted to any longitudinal (i.e. specific to a particular range of magnetic local times) sector.

Most K-family indices suffer from the drawback of low time resolution. The recently developed $\alpha-15$ index is intended to provide a measure of activity similar to am with a 15 minute time resolution [Chambodut et al. (2015)]. Surprisingly, the higher time resolution of $\alpha$-15, when plotted versus $\mathrm{ABI}$ in figure 4.9, did not result in notably smaller error bars or improved linearity (on a semilog scale) with respect to ABI. This could be due to the noise present in ABI, or to the $\alpha-15$ index suffering from a less certain baseline subtraction than in the 3 hour versions, one reason am was not made available at higher cadence Mayaud (1980).

\section{Storm-Time Magnetic Depression Indices versus ABI}

In this section, plots of storm-time magnetic depression, a typically negative quantity with some positive excursions during times of high ram pressure, are desired with the same logarithmic scale used for am above. Accordingly, the index is multiplied by negative one and times negative values are then excluded. In figure $4.10, D_{S T}$ is shown versus ABI. It generally displays linear behavior except during quiet times. The error bars are larger than for am.

We next use the 17 station version of Dcx, obtained from http://dcx.oulu.fi/, which is claimed 
to use better baseline subtraction and station weighting methods to obtain a better measure of storm-time magnetic depression [Mursula et al. (2011)]. In figure 4.11, Dcx is shown versus ABI. We can see that, though error bars are roughly as large as those for $D_{S T}$, the quietest time marker deviates less from a linear relationship than $D_{S T}$, giving credence to the claims of Mursula and his collaborators.

\section{Pressure Corrected Storm-Time Magnetic Depression versus ABI}

$D_{S T}$ and Dcx indices are not purely measures of the ring and tail current systems, but are contaminated by magnetopause currents which vary with ram pressure. In this section, we attempt to use the pressure corrected versions, $D *=D+b \sqrt{P}$, to minimize the effects of magnetopause currents. Different values of the constant, $b$, can be found in the literature, but we use $b=8 n T / \sqrt{n P a}$ from Maltsev (2004) as it is near to that found in the majority of investigations. In figures 4.12 and 4.13 , the corrected versions of $D_{S T}$ and Dcx are respectively shown versus ABI. We see that Dcx* again shows a more linearity than $D_{S T} *$. We overlay the $\mathrm{H}(\mathrm{ABI})$ prediction for tail contribution to Dcx*. The empirically obtained value for tail current contributions to Dcx from Asikainen et al. (2010) is also shown. In figure 4.14, we again lot pressure corrected Dcx*, but with the correction factor of $b=11.84 n T / \sqrt{n P a}$ obtained from fits to the Dcx index as reported by Asikainen et al. (2010). Corrected Dcx* matches well to the theoretical predicted H(ABI).

\section{Sector SMR Indices versus ABI}

The plots for 10-minute averaged SMR indices, obtained from http://supermag.jhuapl.edu/indices/, versus $\mathrm{ABI}$ are shown in figures 4.15 through 4.19. Note that disturbance levels in all sectors increase in magnitude as the Auroral oval expands.

\section{K-family Indices versus Storm-time magnetic depression Indices as Proxies for Auroral Expansion}

In the preceding subsections, we see that plots of indices, on logarithmic scales, do not markedly favor K-family indices when visual comparisons are used. As a follow up, we show correlations and their squares between ABI and the logarithms of the indices considered above. The results, in 
table 4.1, show that K-family indices can account for just of half of the variation in ABI. However, pressure corrected Dcx* can account for nearly as much variation. In table 4.1, the comparisons were made using each $\mathrm{ABI}$ value and its simultaneous index values. In table 4.2, we use the median index values binned per every $2^{\circ}$ of $\mathrm{ABI}$ between $50^{\circ}$ and $70^{\circ}$, which were shown plotted in the aforementioned figures. In this case, correlations between median index values and ABI show that pressure corrected median Dcx* is a better predictor of binned ABI, with more that $99 \%$ of variation accounted for in this data set.

\section{B. AMPERE}

AMPERE is a project of the John's Hopkins University Applied Physics Laboratory (JHU-APL) where commercial magnetometers aboard the roughly 70 Iridium satellites in polar orbits are used to obtain two dimensional maps at a cadence of 10 minutes. They do so by subtracting Earth's magnetic field from the observed field. The magnetic perturbations are then fit to spherical harmonic functions, which are then used (via the Biot-Savart law) to obtain the radial component of field-aligned electric currents.

The capability of AMPERE is unprecedented, but not without its drawbacks. Due to the low sensitivity of the commercial magnetometers, there is considerable uncertainty in the measured currents. Measured currents beneath the threshold of 0.19 micro $A / m^{2}$ magnitude are typically neglected as being beneath the noise in the signal. This makes an accurate determination of FAC currents problematic, especially during quiet times when FAC are relatively weak.

\section{Locating FAC Currents Using AMPERE and K-Means Clustering}

Fortunately, we are not seeking to follow the progress of individual events, but instead wish to determine how strongly related FAC structures are to storm-time geomagnetic activity. Although the typical error in AMPERE provided currents is 0.19 micro $A / m^{2}$, statistical averaging can reduce the effect of random noise such that error reduces as the inverse of the square root of sample size. The AMPERE data set is presently available (with several significant gaps) from 2010 through 2016 with high coverage and after 2016 with degraded coverage. At a 10 minute cadence, this provides us with 297,600 snapshots of the FAC. As we wish to avoid the problem of averaging currents 
with opposite signs, a frequent problem in statistical studies of FAC Stauning et al. (2005), we use k-means clustering to only average spatially similar FAC maps.

k-means clustering works by assigning each observation to a cluster using the criterion of Euclidean distance to the cluster center as a metric Hartigan and Wong (1979). At each iteration, the cluster memberships are updated, followed immediately by an update of the cluster center using the mean of all cluster members. Milan et al. (2015) found that fewer than 200 principle components (i.e. empirical orthogonal basis functions) can account for 90 percent of observed FAC variance. We therefor choose to produce 200 clusters of AMPERE sourced FAC maps for the Northern hemisphere in this study.

\section{Relating Clusters to Geomagnetic Activity}

After the 200 clusters were generated, each cluster member saws associated with its respective values of solar and geomagnetic indices. Statistics were produced for each index for each cluster. It was found that storm-time magnetic depression, Dcx, is in fact strongly associated with the respective clusters. Representative cluster images are shown in figures 4.20 through 4.25. From these representative cluster images, it should be apparent that significant equatorward expansion of the R1 and R2 FAC current regions occurs as Dcx increases in magnitude.

In order to quantify the qualitative behavior seen in figures 4.20 through 4.25 , and to relate this work to the evidence obtained using ABI we need to identify the poleward boundary auroral oval in each cluster. It was found by Ohtani et al. (2010) that the region 2 FAC coincide with the equatorward boundary of the auroral oval. We therefor take 3 Dusk-Dawn meridional slices from each cluster. Automatic identification of current density extrema of the sign appropriate for region 2 currents beyond $15^{\circ}$ magnetic colatitude provided 6 locations along the R2 oval. Examples of these meridional slides along with marked with identified R2 locations for the same clusters in figures 4.20-4.25 are shown in even numbered figures 4.26 through 4.36. Circles were then fit to the $6 \mathrm{R} 2$ locations for each cluster. These circles with the locations from which they were fitted are shown for the aforementioned set of representative clusters in odd numbered figures 4.27 through 4.37. Each fit produced a R2 FAC radius, $\theta$, in degrees, which we take as the radius of the equatorward edge of the auroral oval. To check the automatic identifications, a visual inspection was done on the resulting figures for each cluster. It was found that 6 clusters did not have reasonable R2 
FAC identifications, and in fact did not have easily identifiable current regions even from hand inspections. We therefor exclude those 6 clusters from out following analysis.

We compare Dcx to its theoretical tail current contribution equation from Maltsev (2004), $H(\theta)=-8 / 27 B_{e} \sin (\theta)^{6}$, where $B_{e}$, the strength of Earth's dipole magnetic field at the equator, is taken as $31,000 \mathrm{nT}$, and a constant for the quiet time when $\theta$ is roughly $15^{\circ}$ is subtracted. We plot the median Dcx for each cluster versus $H(\theta)$ in figure 4.38. It should be noted that $H(\theta)$ is often larger than Dcx. This is at first surprising since other sources, such as ring current, also contribute to Dcx. However, when the pressure corrected version of Dcx found by Asikainen et al. (2010), $D c x *=D c x-11.84 n T / \operatorname{sqrt}(n P a) \operatorname{sqrt}(P)$, is applied (see figure 4.39) we see that apparent over-prediction is due in large part to the effect of magnetopause currents upon Dcx. For the cluster with the largest median Dcx magnitude, we see that some over-prediction remains. This is likely due to the paucity of extremely disturbed times in the data set leading to questionable statistics for that cluster, as many FAC maps from many different disturbance levels contributed to that cluster. Another likely contributing factor is the erosion of the day-side magnetosphere during periods of strong solar wind energy transfer, which brings the magnetopause closer to Earth, requiring a stronger pressure correction during these disturbed intervals [Siscoe et al. (2005)].

\section{Summary and Conclusions}

In this chapter, with the goal of testing the plausibility of Maltsev's theoretically predicted tail contribution to storm-time magnetic depression, we have compared indices measuring storm-time magnetic depression to measurements of the auroral oval latitude. As the most widely available time series running for several years is the auroral boundary index, long known to be linearly associated with the quasi-logarithmic Kp, we first set out to determine if indices of storm strength can also show linear relationships to ABI. We found that the relationships of storm strength indices are, in fact, linearly related to ABI. We also found that Matsev's formula for tail contribution, using an estimate of auroral oval size from ABI, coincides with pressure corrected Dcx index. We also see that Dcx displays a cleaner relation to ABI that other storm-time indices, giving credence to claims that Dcx is an improved version of $D_{S T}$.

We also used field aligned current maps for the Northern hemisphere provided by the AMPERE 
project to determine the extent of the auroral oval. The limitations of the noisy AMPERE data set were mitigated by statistically averaging many similar FAC maps together, and we insured that only those FAC maps with similar spatial arrangement were averaged by using k-means clustering. When the theoretically predicted tail contribution, $H(\theta)$, is compared to the median Dcx* for each cluster, it was found that predicted tail current contributions match the measured disturbance well, suggesting that the magnetotail can account for much of the measured storm-time magnetic depression.

This conclusion has been supported by some previous investigations (e.g. Lopez et al. (2015) and Alexeev et al. (1996)). However, there are still papers being published adhering to the (likely incorrect) view that $D_{S T}$ is simply a ring current proxy. In some such studies (e.g. O'Brien and McPherron (2000)), this is merely a semantic issue, but sometime leads to deficient interpretations of the data. 


\section{References}

Alexeev, I., E. Belenkaya, V. Kalegaev, Y. I. Feldstein, and A. Grafe (1996), Magnetic storms and magnetotail currents, Journal of Geophysical Research: Space Physics, 101(A4), 7737-7747.

Arykov, A., and Y. P. Maltsev (1996), Direct-driven mechanism for geomagnetic storms, Geophysical research letters, 23(13), 1689-1692.

Asikainen, T., V. Maliniemi, and K. Mursula (2010), Modeling the contributions of ring, tail, and magnetopause currents to the corrected dst index, Journal of Geophysical Research: Space Physics, 115(A12).

Baumjohann, W. (1986), Merits and limitations of the use of geomagnetic indices in solar wind-magnetosphere coupling studies, in Solar Wind Magnetosphere Coupling, Astrophysics and Space Science Library, vol. 126, edited by Y. Kamide and J. A. Slavin, pp. 3-15, doi: 10.1007/978-90-277-2303-1_1.

Birdsall, C. K., and A. B. Langdon (2004), Plasma physics via computer simulation, CRC press.

Borovsky, J. E. (2013), Physical improvements to the solar wind reconnection control function for the earth's magnetosphere, Journal of Geophysical Research: Space Physics, 118(5), 2113-2121.

Borovsky, J. E., and J. A. Valdivia (2018), The earths magnetosphere: A systems science overview and assessment, Surveys in Geophysics, 39(5), 817-859.

Boynton, R. J., M. A. Balikhin, S. A. Billings, H.-L. Wei, and N. Ganushkina (2011), Using the narmax ols-err algorithm to obtain the most influential coupling functions that affect the evolution of the magnetosphere, Journal of Geophysical Research: Space Physics, 116(A5). 


\section{REFERENCES}

Büchner, J. (2005), Vlasov-code simulation, in Proceedings of ISSS, vol. 7, pp. 26-31.

Burton, R. K., R. L. McPherron, and C. T. Russell (1975), An empirical relationship between interplanetary conditions and dst, Journal of Geophysical Research, 80(31), 4204-4214, doi: 10.1029/JA080i031p04204.

Chambodut, A., A. Marchaudon, M. Menvielle, F. E.-L. Mazouz, and C. Lathuillère (2013), The k-derived mlt sector geomagnetic indices, Geophysical Research Letters, 40(18), 4808-4812.

Chambodut, A., A. Marchaudon, C. Lathuillère, M. Menvielle, and E. Foucault (2015), New hemispheric geomagnetic indices $\alpha$ with 15 min time resolution, Journal of Geophysical Research: Space Physics, 120(11), 9943-9958.

Cliver, E. W., and L. Svalgaard (2004), The 1859 solar-terrestrial disturbance and the current limits of extreme space weather activity, Solar Physics, 224(1), 407-422, doi:10.1007/ s11207-005-4980-z.

Coleman, I. J., G. Chisham, M. Pinnock, and M. P. Freeman (), An ionospheric convection signature of antiparallel reconnection, Journal of Geophysical Research: Space Physics, 106(A12), 28,99529,007, doi:10.1029/2001JA900084.

Corana, A., M. Marchesi, C. Martini, and S. Ridella (1987), Minimizing multimodal functions of continuous variables with the simulated annealing algorithm corrigenda for this article is available here, ACM Transactions on Mathematical Software (TOMS), 13(3), 262-280.

Daglis, I., Y. Kamide, C. Monikis, G. Reeves, E. Sarris, K. Shiokawa, and B. Wilken (2000), fine structure of the storm-substorm relationship: Ion injections during dst decrease, Advances in Space Research, 25(12), 2369-2372.

Daglis, I. A., R. M. Thorne, W. Baumjohann, and S. Orsini (1999), The terrestrial ring current: Origin, formation, and decay, Reviews of Geophysics, 37(4), 407-438.

Detman, T. R., and D. Vassiliadis (1997), Review of Techniques for Magnetic Storm Forecasting, pp. 253-266, American Geophysical Union (AGU), doi:10.1029/GM098p0253. 


\section{REFERENCES}

Dielman, T. E. (1986), A comparison of forecasts from least absolute value and least squares regression, Journal of Forecasting, 5(3), 189-195, doi:10.1002/for.3980050305.

Feldstein, Y., and G. Starkov (1967), Dynamics of auroral belt and polar geomagnetic disturbances, Planetary and Space Science, 15(2), 209-229.

Feldstein, Y. I. (1992), Modelling of the magnetic field of magnetospheric ring current as a function of interplanetary medium parameters, Space Science Reviews, 59(1), 83-165, doi: 10.1007/BF01262538.

Feldstein, Y. I., L. Dremukhina, U. Mall, and J. Woch (2000), On the two-phase decay of the dst-variation, Geophysical research letters, 27(17), 2813-2816.

Fogel, L. J. (1999), Intelligence through simulated evolution: forty years of evolutionary programming, John Wiley \& Sons, Inc.

Friedman, J., T. Hastie, and R. Tibshirani (2001), The elements of statistical learning, vol. 1, Springer series in statistics New York, NY, USA:

Fuselier, S., S. Petrinec, and K. Trattner (2010), Antiparallel magnetic reconnection rates at the earth's magnetopause, Journal of Geophysical Research: Space Physics, 115(A10).

Ganushkina, N. Y., T. Pulkkinen, M. Kubyshkina, H. Singer, and C. Russell (2004), Long-term evolution of magnetospheric current systems during storms, in Annales Geophysicae, vol. 22, pp. $1317-1334$.

Ganushkina, N. Y., et al. (2015), Defining and resolving current systems in geospace, Annales Geophysicae, 33(11), 1369-1402, doi:10.5194/angeo-33-1369-2015.

Garrett, H., A. Dessler, and T. W. Hill (1974), Influence of solar wind variability on geomagnetic activity, Journal of Geophysical Research, 79(31), 4603-4610.

Goldberg, D. E., et al. (1989), Genetic algorithms in search, optimization, and machine learning, Addison-Wesley Pub. Co. 


\section{REFERENCES}

Greenspan, M. E., and D. C. Hamilton (2000), A test of the dessler-parker-sckopke relation during magnetic storms, Journal of Geophysical Research: Space Physics, 105(A3), 5419-5430, doi: 10.1029/1999JA000284.

Gussenhoven, M., D. A. Hardy, N. Heinemann, and E. Holeman (1982), 1978 diffuse auroral boundaries and a derived auroral boundary index, Tech. rep., AIR FORCE GEOPHYSICS LAB HANSCOM AFB MA.

Gussenhoven, M., D. Hardy, and N. Heinemann (1983), Systematics of the equatorward diffuse auroral boundary, Journal of Geophysical Research: Space Physics, 88(A7), 5692-5708.

Gvozdevsky, B., and V. Sergeev (1996), Mt-index a possible new index to characterize the configuration of the magnetotail, Advances in Space Research, 18(8), 51 - 54, doi:https://doi.org/10. 1016/0273-1177(95)00995-7, the Three-dimensional Magnetosphere.

Hamilton, D. C., G. Gloeckler, F. Ipavich, W. Stüdemann, B. Wilken, and G. Kremser (1988), Ring current development during the great geomagnetic storm of february 1986, Journal of Geophysical Research: Space Physics, 93(A12), 14,343-14,355.

Hartigan, J. A., and M. A. Wong (1979), Algorithm as 136: A k-means clustering algorithm, Journal of the Royal Statistical Society. Series C (Applied Statistics), 28(1), 100-108.

Iijima, T., and T. A. Potemra (1976), Field-aligned currents in the dayside cusp observed by triad, Journal of Geophysical Research, 81(34), 5971-5979, doi:10.1029/JA081i034p05971.

Iyemori, T., and D. R. K. Rao (1996), Decay of the dst field of geomagnetic disturbance after substorm onset and its implication to storm-substorm relation, Annales Geophysicae, 14(6), 608-618, doi:10.1007/s00585-996-0608-3.

Iyemori, T., H. Maeda, and T. Kamei (1979), Impulse response of geomagnetic indices to interplanetary magnetic field, Journal of geomagnetism and geoelectricity, 31(1), 1-9, doi:10.5636/jgg.31.1.

Johnsen, M. G. (2013), Real-time determination and monitoring of the auroral electrojet boundaries, Journal of Space Weather and Space Climate, 3, A28. 


\section{REFERENCES}

Kamide, Y., and Y. P. Maltsev (2007), Geomagnetic storms, Handbook of the Solar-Terrestrial Environment, pp. 355-374.

Kan, J., and L. Lee (1979), Energy coupling function and solar wind-magnetosphere dynamo, Geophysical Research Letters, 6(7), 577-580.

Kavosi, S., and J. Raeder (2015), Ubiquity of kelvin-helmholtz waves at earths magnetopause, Nature Communications, 6, 7019.

Kepko, L., et al. (2015), Substorm current wedge revisited, Space Science Reviews, 190(1-4), 1-46.

Kivelson, M. G., and C. T. Russell (1995), Introduction to Space Physics, Cambridge University Press.

Knipp, D. J., B. J. Fraser, M. Shea, and D. Smart (2018), On the little-known consequences of the 4 august 1972 ultra-fast coronal mass ejecta: Facts, commentary and call to action, Space Weather.

Koza, J. (1992), Genetic Programming MIT Press.

Lopez, R., W. Gonzalez, V. Vasyliūnas, I. Richardson, C. Cid, E. Echer, G. Reeves, and P. Brandt (2015), Decrease in sym-h during a storm main phase without evidence of a ring current injection, Journal of Atmospheric and Solar-Terrestrial Physics, 134, 118-129.

Lui, A., R. McEntire, S. Krimigis, and E. Keath (1986), Acceleration of energetic oxygen (ei 137 kev) in the storm-time ring current, Ion acceleration in the magnetosphere and ionosphere, 38, $149-152$.

Madden, D., and M. S. Gussenhoven (1990), Auroral boundary index from 1983 to 1990, Tech. rep.

Maltsev, Y. P. (1996), Storm and substorm in terms of the magnetic flux redistribution, in International Conference on Substorms, vol. 389, p. 675.

Maltsev, Y. P. (2004), Points of controversy in the study of magnetic storms, Space Science Reviews, $110(3-4), 227-267$.

Maltsev, Y. P., and B. Rezhenov (2003), Relation of dst index to solar wind parameters, Int. J. Geomagn. Aeron, 4(1), 1-9. 


\section{REFERENCES}

Maltsev, Y. P., A. Arykov, E. Belova, B. Gvozdevsky, and V. Safargaleev (1996), Magnetic flux redistribution in the storm time magnetosphere, Journal of Geophysical Research: Space Physics, $101(\mathrm{~A} 4), 7697-7704$.

Maus, S., and P. Weidelt (2004), Separating the magnetospheric disturbance magnetic field into external and transient internal contributions using a 1d conductivity model of the earth, Geophysical Research Letters, 31(12), doi:10.1029/2004GL020232.

Mayaud (1980), Derivation, Meaning, and Use of Geomagnetic Indices, 1 ed., American Geophysical Union (AGU), doi:10.1029/GM022.

McPherron, R. L., C. T. Russell, and M. P. Aubry (1973), Satellite studies of magnetospheric substorms on august 15, 1968: 9. phenomenological model for substorms, Journal of Geophysical Research, $78(16), 3131-3149$.

Milan, S., J. Gosling, and B. Hubert (2012), Relationship between interplanetary parameters and the magnetopause reconnection rate quantified from observations of the expanding polar cap, Journal of Geophysical Research: Space Physics, 117(A3).

Milan, S. E., J. Carter, H. Korth, and B. Anderson (2015), Principal component analysis of birkeland currents determined by the active magnetosphere and planetary electrodynamics response experiment, Journal of Geophysical Research: Space Physics, 120(12), 10-415.

Moon, G.-H., B.-H. Ahn, and W. Sun (2006), Estimation of the dst index based on the al index, Advances in Space Research, 37(6), 1148-1151.

Moore, T., et al. (1999), Ionospheric mass ejection in response to a cme, Geophysical Research Letters, 26(15), 2339-2342.

Murayama, T. (1986), Coupling function between the solar wind and the Dst index, in Solar Wind Magnetosphere Coupling, Astrophysics and Space Science Library, vol. 126, edited by Y. Kamide and J. A. Slavin, pp. 119-126, doi:10.1007/978-90-277-2303-1_9.

Mursula, K., L. Holappa, and A. Karinen (2011), Uneven weighting of stations in the dst index, Journal of Atmospheric and Solar-Terrestrial Physics, 73(2), 316 - 322, doi:https://doi.org/10. 1016/j.jastp.2010.04.007, space Climate. 


\section{REFERENCES}

Nakayama, Y., Y. Ebihara, M. Fok, and T. Tanaka (2017), Impact of substorm time o+ outflow on ring current enhancement, Journal of Geophysical Research: Space Physics, 122(6), 6304-6317.

Newell, P., and J. Gjerloev (2011), Evaluation of supermag auroral electrojet indices as indicators of substorms and auroral power, Journal of Geophysical Research: Space Physics, 116(A12).

Newell, P., T. Sotirelis, K. Liou, C.-I. Meng, and F. Rich (2006), Cusp latitude and the optimal solar wind coupling function, Journal of Geophysical Research: Space Physics, 111(A9).

Newell, P., T. Sotirelis, K. Liou, C.-I. Meng, and F. Rich (2007), A nearly universal solar windmagnetosphere coupling function inferred from 10 magnetospheric state variables, Journal of Geophysical Research: Space Physics, 112(A1).

Newell, P. T., and J. W. Gjerloev (), Supermag-based partial ring current indices, Journal of Geophysical Research: Space Physics, 117(A5), doi:10.1029/2012JA017586.

Newell, P. T., V. A. Sergeev, G. R. Bikkuzina, and S. Wing (1998), Characterizing the state of the magnetosphere: Testing the ion precipitation maxima latitude (b2i) and the ion isotropy boundary, Journal of Geophysical Research: Space Physics, 103(A3), 4739-4745, doi:10.1029/ 97JA03622.

Newell, P. T., T. Sotirelis, K. Liou, and F. Rich (2008), Pairs of solar wind-magnetosphere coupling functions: Combining a merging term with a viscous term works best, Journal of Geophysical Research: Space Physics, 113(A4).

Nosé, M., H. Koshiishi, H. Matsumoto, K. Keika, K. Koga, T. Goka, T. Obara, et al. (2010), Magnetic field dipolarization in the deep inner magnetosphere and its role in development of o+-rich ring current, Journal of Geophysical Research: Space Physics, 115(A9).

O'Brien, P., and R. L. Mcpherron (), An empirical phase space analysis of ring current dynamics: Solar wind control of injection and decay, Journal of Geophysical Research: Space Physics, 105(A4), 7707-7719, doi:10.1029/1998JA000437.

O'Brien, T., and R. L. McPherron (2000), Forecasting the ring current index dst in real time, Journal of Atmospheric and Solar-Terrestrial Physics, 62(14), 1295 - 1299, doi:https://doi.org/ 10.1016/S1364-6826(00)00072-9, space Weather Week. 


\section{REFERENCES}

O'Brien, T. P., and R. L. McPherron (), Evidence against an independent solar wind density driver of the terrestrial ring current, Geophysical Research Letters, 27(23), 3797-3799, doi:10.1029/ 2000GL012125.

Ohtani, S., M. Nos, G. Rostoker, H. Singer, A. T. Y. Lui, and M. Nakamura (2001), Stormsubstorm relationship: Contribution of the tail current to dst, Journal of Geophysical Research: Space Physics, 106(A10), 21,199-21,209, doi:10.1029/2000JA000400.

Ohtani, S., P. Brandt, D. Mitchell, H. Singer, M. Nosé, G. Reeves, and S. Mende (2005), Stormsubstorm relationship: Variations of the hydrogen and oxygen energetic neutral atom intensities during storm-time substorms, Journal of Geophysical Research: Space Physics, 110(A7).

Ohtani, S., S. Wing, P. Newell, and T. Higuchi (2010), Locations of night-side precipitation boundaries relative to r2 and r1 currents, Journal of Geophysical Research: Space Physics, 115(A10).

Papitashvili, V., F. Christiansen, and T. Neubert (2002), A new model of field-aligned currents derived from high-precision satellite magnetic field data, Geophysical Research Letters, 29(14), $28-1$.

Park, K. S., T. Ogino, and R. J. Walker (), On the importance of antiparallel reconnection when the dipole tilt and imf by are nonzero, Journal of Geophysical Research: Space Physics, 111(A5), doi:10.1029/2004JA010972.

Parks, G. K. (1991), Physics of space plasmas - an introduction.

Perreault, P., and S.-I. Akasofu (1978), A study of geomagnetic storms, Geophysical Journal of the Royal Astronomical Society, 54(3), 547-573.

Perrone, L., and G. D. Franceschi (1998), Solar, ionospheric and geomagnetic indices, Annals of Geophysics, 41(5-6), doi:10.4401/ag-3824.

Pisarskij, V. Y., Y. I. Feldstein, N. Rudneva, A. Prigancova, and L. Tř́sková (1989), Ring current and interplanetary medium parameters, Studia geophysica et geodaetica, 33(1), 61-80.

Press, W., S. Teukolsky, W. Vetterling, and B. Flannery (1992), Numerical Recipes in FORTRAN 2nd ed., Cambridge Univ. Press, New York. 


\section{REFERENCES}

Roeder, J., J. Fennell, M. Chen, M. Schulz, M. Grande, and S. Livi (1996), Crres observations of the composition of the ring-current ion populations, Advances in Space Research, 17(10), 17-24.

Russell, C. T., Y. L. Wang, and J. Raeder (2003), Possible dipole tilt dependence of dayside magnetopause reconnection, Geophysical Research Letters, 30(18), doi:10.1029/2003GL017725.

Schulz, M. (1997), Direct influence of ring current on auroral oval diameter, Journal of Geophysical Research: Space Physics, 102(A7), 14,149-14,154.

Schwefel, H.-P., H.-P. Schwefel, H.-P. Schwefel, and H.-P. Schwefel (1995), Evolution and optimum seeking, vol. 1515, Wiley New York.

Scurry, L., and C. Russell (1991), Proxy studies of energy transfer to the magnetosphere, Journal of Geophysical Research: Space Physics, 96(A6), 9541-9548.

Siscoe, G. L., R. L. McPherron, and V. K. Jordanova (2005), Diminished contribution of ram pressure to dst during magnetic storms, Journal of Geophysical Research: Space Physics, 110(A12), doi:10.1029/2005JA011120.

Sonnerup, B. Ö. (1974), Magnetopause reconnection rate, Journal of Geophysical Research, 79(10), $1546-1549$.

Stauning, P., F. Christiansen, and J. Watermann (2005), On the modelling of field-aligned currents from magnetic observations by polar orbiting satellites, in Earth Observation with CHAMP, pp. 371-374, Springer.

Stern, D. P. (1983), The origins of birkeland currents, Reviews of Geophysics, 21(1), 125-138.

Svalgaard, L. (1977), Geomagnetic activity: Dependence on solar wind parameters., Tech. rep., STANFORD UNIV CALIF INST FOR PLASMA RESEARCH.

Tajima, T. (2018), Computational plasma physics: with applications to fusion and astrophysics, CRC Press.

Thomsen, M. F. (2004), Why kp is such a good measure of magnetospheric convection, Space Weather, 2(11), doi:10.1029/2004SW000089. 
Tsyganenko, N. (1989), A magnetospheric magnetic field model with a warped tail current sheet, Planetary and Space Science, 37(1), 5-20.

Turner, N., T. Pulkkinen, D. Baker, and R. McPherron (2002), 1. intensity of the cross-tail current, JOURNAL OF GEOPHYSICAL RESEARCH, 107(A1).

Vassiliadis, D., A. Klimas, J. Valdivia, and D. Baker (1999), The dst geomagnetic response as a function of storm phase and amplitude and the solar wind electric field, Journal of Geophysical Research: Space Physics, 104(A11), 24,957-24,976.

Vasyliunas, V. M. (), Reinterpreting the burton-mcpherron-russell equation for predicting dst, Journal of Geophysical Research: Space Physics, $111($ A7), doi:10.1029/2005JA011440.

Vasyliunas, V. M., J. R. Kan, G. L. Siscoe, and S.-I. Akasofu (1982), Scaling relations governing magnetospheric energy transfer, Planetary and Space Science, 30(4), 359-365.

Vasylinas, V. M. (2006), Ionospheric and boundary contributions to the dessler-parker-sckopke formula for dst, Annales Geophysicae, 24(3), 1085-1097, doi:10.5194/angeo-24-1085-2006.

Veselovsky, I., A. Dmitriev, and A. Suvorova (2010), Algebra and statistics of the solar wind, Cosmic Research, 48(2), 113-128.

Weigel, R. S. (2010), Solar wind density influence on geomagnetic storm intensity, Journal of Geophysical Research: Space Physics, 115(A9), doi:10.1029/2009JA015062.

Wu, J.-G., and H. Lundstedt (1996), Prediction of geomagnetic storms from solar wind data using elman recurrent neural networks, Geophysical research letters, 23(4), 319-322.

Wygant, J. R., R. B. Torbert, and F. Mozer (1983), Comparison of s3-3 polar cap potential drops with the interplanetary magnetic field and models of magnetopause reconnection, Journal of Geophysical Research: Space Physics, 88(A7), 5727-5735. 


\section{Tables}




\begin{tabular}{|c|c|c|c|}
\hline \hline Station & Geographic Latitude & Geographic Longitude & Magnetic Dipole Latitude \\
\hline Hermanus & 19.22 & -34.40 & -33.3 \\
Kakioka & 140.18 & 36.23 & 26.0 \\
Honolulu (to April 1960) & 201.90 & 21.30 & 21.0 \\
Honolulu (after April 1960) & 201.98 & 21.32 & 21.1 \\
San Juan (to Jan. 1965) & 293.88 & 18.38 & 29.9 \\
San Juan (after Jan. 1965) & 293.88 & 18.11 & 28.0 \\
\hline
\end{tabular}

Table 1.1: A listing of magnetic observatory stations used to compute $D_{S T}$. From the World geomagnetic Data Center at Kyoto (http://wdc.kugi.kyoto-u.ac.jp).

\begin{tabular}{|c|c|c|c|}
\hline \hline Station & Code & Magnetic Latitude & Magnetic Longitude \\
\hline San Juan & SJG & 28.04 & 6.54 \\
Fredericksburg & FRD & 48.14 & 353.93 \\
Boulder & BOU & 48.24 & 321.28 \\
Tucson & TUC & 39.73 & 316.74 \\
Honolulu & HON & 21.71 & 270.27 \\
Memambetsu & MMB & 35.63 & 211.74 \\
Urumqi & WMQ & 34.34 & 162.53 \\
Alibag & ABG & 10.37 & 146.55 \\
Martin de Vivies & AMS & -46.22 & 144.93 \\
Hermanus & HER & -34.08 & 84.63 \\
Chambon-la-Foret & CLF & 49.75 & 85.80 \\
\hline
\end{tabular}

Table 1.2: A listing of magnetic observatory stations used to compute SYM/H. From the World geomagnetic Data Center at Kyoto (http://wdc.kugi.kyoto-u.ac.jp). 


\begin{tabular}{|cccccccc|}
\hline \hline IAGA Code & Name & GGLat & GGLon & GMLat & Elev & Dcx & Dcx17 \\
\hline AAA & Alma Ata & 43.180 & 76.920 & 34.429 & 1300 & & $\mathrm{x}$ \\
BSL & Stennis & 30.350 & 270.360 & 39.821 & 8 & & $\mathrm{x}$ \\
CNB & Canberra & -35.315 & 149.363 & -42.466 & 859 & & $\mathrm{x}$ \\
CTO & Cape Town & -33.950 & 18.467 & -32.540 & 26 & $\mathrm{x}$ & \\
GNA & Gnangara & -31.780 & 115.947 & -41.651 & 60 & & $\mathrm{x}$ \\
HBK & Hartebeesthoek & -25.882 & 27.707 & -27.181 & 1555 & & $\mathrm{x}$ \\
HER & Hermanus & -34.425 & 19.225 & -34.081 & 26 & $\mathrm{x}$ & $\mathrm{x}$ \\
HON & Honolulu & 21.320 & 202.000 & 21.713 & 4 & $\mathrm{x}$ & $\mathrm{x}$ \\
IZN & Iznik & 40.500 & 29.720 & 37.757 & 256 & & $\mathrm{x}$ \\
KAK & Kakioka & 36.232 & 140.186 & 27.647 & 36 & $\mathrm{x}$ & $\mathrm{x}$ \\
LZH & Lanzhou & 36.087 & 103.845 & 26.123 & 1560 & & $\mathrm{x}$ \\
MBO & Mbour & 14.380 & 343.030 & 19.897 & 7 & & $\mathrm{x}$ \\
PPT & Pamatai & -17.567 & 210.426 & -15.133 & 357 & & $\mathrm{x}$ \\
SJG & San Juan & 18.117 & 293.850 & 28.042 & 424 & $\mathrm{x}$ & $\mathrm{x}$ \\
TAM & Tamanrasset & 22.793 & 5.532 & 24.547 & 1373 & & $\mathrm{x}$ \\
TRW & Trelew & -43.267 & 294.617 & -33.343 & 15 & & $\mathrm{x}$ \\
TUC & Tucson & 32.170 & 249.270 & 39.733 & 946 & & $\mathrm{x}$ \\
VSS & Vassouras & -22.400 & 316.350 & -13.563 & 457 & & $\mathrm{x}$ \\
\hline
\end{tabular}

Table 1.3: A listing of magnetic observatory stations used to compute Dcx index. From the dcx.oulu.fi.

\begin{tabular}{|c|c|c|c|}
\hline \hline Station & Code & Magnetic Latitude & Magnetic Longitude \\
\hline San Juan & SJG & 28.04 & 6.54 \\
Fredericksburg & FRD & 48.14 & 353.93 \\
Boulder & BOU & 48.24 & 321.28 \\
Tucson & TUC & 39.73 & 316.74 \\
Honolulu & HON & 21.71 & 270.27 \\
Memambetsu & MMB & 35.63 & 211.74 \\
Urumqi & WMQ & 34.34 & 162.53 \\
Alibag & ABG & 10.37 & 146.55 \\
Martin de Vivies & AMS & -46.22 & 144.93 \\
Hermanus & HER & -34.08 & 84.63 \\
Chambon-la-Foret & CLF & 49.75 & 85.80 \\
\hline
\end{tabular}

Table 1.2: A listing of magnetic observatory stations used to compute SYM/H. From the World geomagnetic Data Center at Kyoto (http://wdc.kugi.kyoto-u.ac.jp). 


\begin{tabular}{|c|c|}
\hline \hline SWCF & Source \\
\hline$v B_{S}$ & Burton et al. $(1975)$ \\
$d \Phi_{M P} / d t=v^{4 / 3} B_{T}^{2 / 3} \sin ^{8 / 3}\left(\theta_{C} / 2\right)$ & Newell et al. (2007) \\
$\Phi_{D}=v^{4 / 3} B_{T} \sin ^{9 / 2}\left(\theta_{C} / 2\right)$ & Milan et al. $(2012)$ \\
$E_{W A V}=v B_{T} \sin ^{4}\left(\theta_{C} / 2\right)$ & Wygant et al. (1983) \\
$E_{V}=N^{1 / 6} v^{5 / 3} B_{T} \sin ^{4}\left(\theta_{C} / 2\right)$ & Vasyliunas et al. (1982) \\
$E_{K L}=v B_{T} \sin ^{2}\left(\theta_{C} / 2\right)$ & Kan and Lee (1979) \\
$E_{S R}=N^{1 / 2} v B_{T}^{2} \sin ^{4}\left(\theta_{C} / 2\right)$ & Scurry and Russell $(1991)$ \\
$\varepsilon=v B_{T}^{2} \sin ^{4}\left(\theta_{C} / 2\right)$ & Perreault and Akasofu (1978) \\
\hline
\end{tabular}

Table 2.1: Some commonly encountered solar wind coupling functions are shown. In the above, $\theta_{C}=\arctan \left(B_{Y} / B_{Z}\right)$ is IMF clock angle, $N$ is SW proton density, $v$ is the (anti-sunward) SW speed, $B_{S}$ is the rectified IMF componant directed in the -Z GSM direction, and $B_{T}$ is the IMF in the plane perpendicular to the Earth-Sun line. 


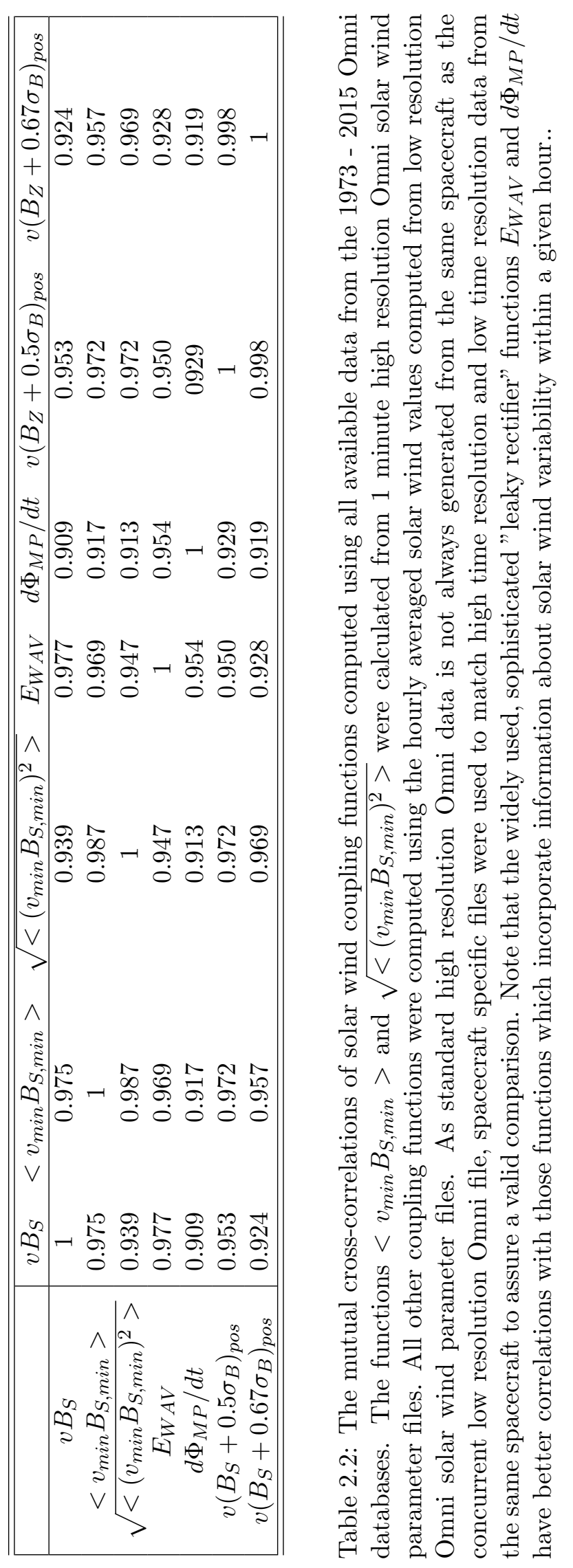




\begin{tabular}{|ccc|}
\hline \hline Index & Number of Hours of IMF & $\mathrm{w}$ \\
\hline Cusp latitude & 1 & 1.0 \\
$D_{S T}$ & 72 & 0.95 \\
$\mathrm{AE}$ & 3 & 0.69 \\
$\mathrm{AU}$ & 3 & 0.77 \\
Goes tilt & 5 & 0.78 \\
Kp & 6 & $\mathrm{n} / \mathrm{a}$ \\
Auroral Power & 4 & 0.65 \\
b2i & 6 & 0.76 \\
Polar Cap Magnetic Flux & 7 & 0.57 \\
AL & 3 & 0.69 \\
\hline \hline
\end{tabular}

Table 2.3: Table 4 from Newell et al. (2007). The original caption reads: "For example, Dst is best predicted by integrating over 72 hours of IMF data, with the hour n-hours previous to the present receiving relative weightof $0.95 \mathrm{n}$. Kp and cusp latitude had to be treated somewhat differently."

\begin{tabular}{|c|ccccc|}
\hline \hline SWCF & $Q_{D_{S} T}$ & $\mathrm{AE}$ & $\mathrm{AU}$ & $\mathrm{AL}$ & $\mathrm{PC}(\mathrm{N})$ \\
\hline$v B_{S}$ & $48.2 \%$ & $51.8 \%$ & $31.7 \%$ & $52.1 \%$ & $44.5 \%$ \\
$<v_{\min } B_{S, \text { min }}>$ & $51.6 \%$ & $56.1 \%$ & $35.7 \%$ & $55.7 \%$ & $48.9 \%$ \\
$\sqrt{<\left(v_{\min } B_{S, \min }\right)^{2}>}$ & $52.4 \%$ & $59.1 \%$ & $39.1 \%$ & $57.7 \%$ & $52.0 \%$ \\
$E_{W A V}{ }_{d}$ & $49.6 \%$ & $55.0 \%$ & $35.7 \%$ & $54.1 \%$ & $50.8 \%$ \\
$d \Phi_{M P} / d t$ & $46.5 \%$ & $67.1 \%$ & $46.8 \%$ & $64.1 \%$ & $60.1 \%$ \\
$v\left(B_{S}+0.5 \sigma_{B}\right)_{\text {pos }}$ & $52.3 \%$ & $60.7 \%$ & $40.2 \%$ & $59.2 \%$ & $52.0 \%$ \\
$v\left(B_{S}+0.67 \sigma_{B}\right)_{\text {pos }}$ & $52.3 \%$ & $61.9 \%$ & $41.9 \%$ & $59.9 \%$ & $52.9 \%$ \\
\hline \hline
\end{tabular}

Table 2.4: The percent variance (i.e. squared correlation) of geomagnetic indices predicted by the solar wind coupling functions listed using all available data from 1973 - 2015 Omni databases. The functions $\left\langle v_{\min } B_{S, \min }>\right.$ and $\sqrt{\left\langle\left(v_{\min } B_{S, \min }\right)^{2}\right\rangle}$ were calculated from 1 minute high resolution Omni solar wind parameter files. All other coupling functions were computed using the hourly averaged solar wind values computed from low resolution Omni solar wind parameter files. As standard high resolution Omni data is not always generated from the same spacecraft as the concurrent low resolution Omni file, spacecraft specific files were used to match high time resolution and low time resolution data from the same spacecraft to assure a valid comparison. Coupling functions for AE, $\mathrm{AU}$, and $\mathrm{AL}$ were correlated to the 3 hour weighted average according, weighted as described in Newell et al. (2007). $Q_{D_{S T}}$ is the injection function for the $D_{S T}$ model of Burton et al. (1975) using the original parameters from the same paper. Note that the RMS value of 1-minute $v B_{S}$ is the best predictor of $D_{S T}$ "injection", with other functions considering SW variability also performing well. For other indices, Newell's coupling function, $d \Phi_{M P} / d t$, derived empirically to match these indices, is the best predictor. However, functions considering SW variability outperform all other functions. Note also that the RMS value of 1-minute $v B_{S}$ out performs the mean value of 1-minute $v B_{S}$ as an index predictor in all cases. 


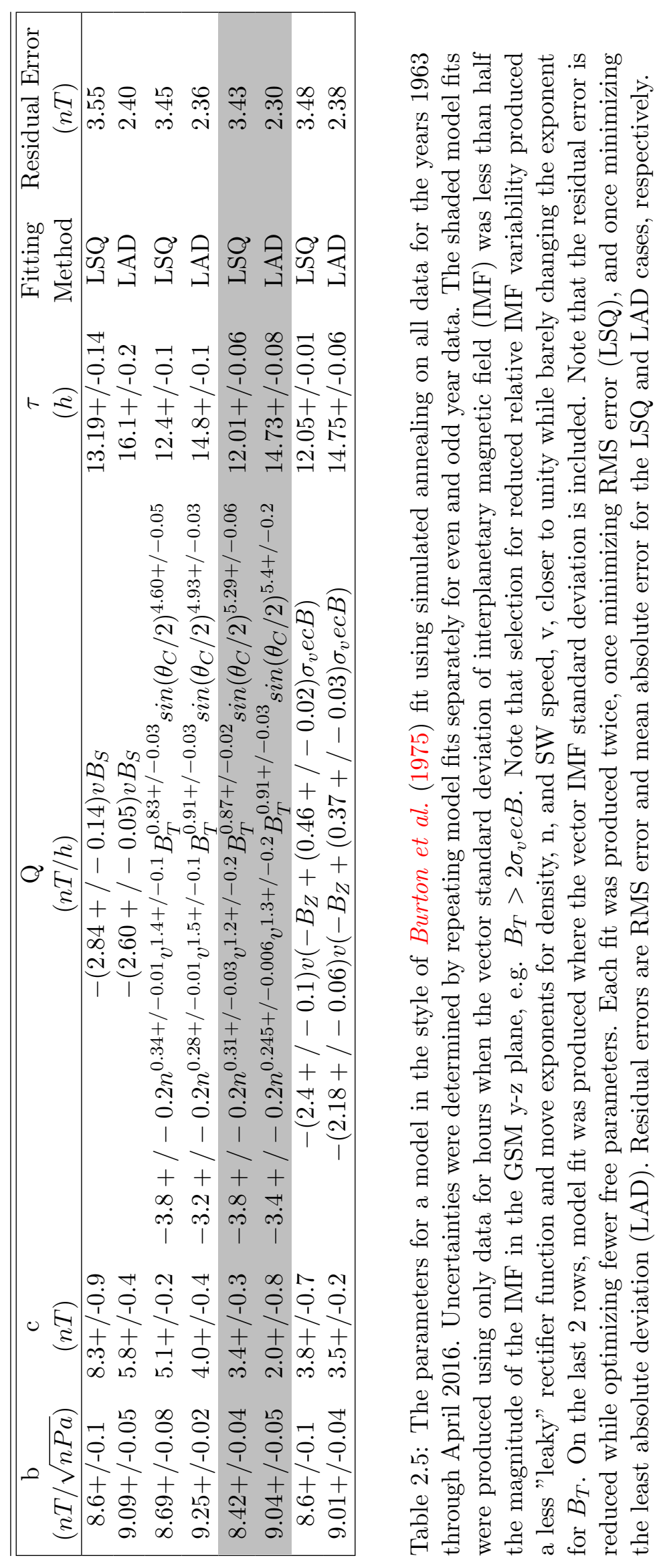




\begin{tabular}{|c|cc|}
\hline \hline & $|R|$ & $R^{2}$ \\
\hline $\log (1+a p)$ & 0.716 & $51.2 \%$ \\
$\log (1+a m)$ & 0.717 & $51.4 \%$ \\
$\log (1+a m 00)$ & 0.702 & $49.3 \%$ \\
$\log (1+a m 06)$ & 0.711 & $50.5 \%$ \\
$\log (1+a m 12)$ & 0.682 & $46.5 \%$ \\
$\log (1+a m 18)$ & 0.711 & $48.6 \%$ \\
$\log \left(1-D_{S T, n e g}\right)$ & 0.718 & $51.6 \%$ \\
$\log \left(1-D c x_{\text {neg }}\right)$ & 0.558 & $31.1 \%$ \\
$\log \left(1-S M R_{\text {neg }}\right)$ & 0.583 & $34.0 \%$ \\
$\log \left(1-S M R 00_{\text {neg }}\right)$ & 0.526 & $34.6 \%$ \\
$\log \left(1-S M R 06_{\text {neg }}\right)$ & 0.456 & $20.8 \%$ \\
$\log \left(1-S M R 12_{\text {neg }}\right)$ & 0.549 & $30.2 \%$ \\
$\log \left(1-S M R 18_{\text {neg }}\right)$ & 0.621 & $38.6 \%$ \\
$\log \left(100-D_{S T}\right)$ & 0.605 & $36.6 \%$ \\
$\log \left(100-D_{c x}\right)$ & 0.602 & $36.3 \%$ \\
$\log (300-S M R)$ & 0.595 & $35.4 \%$ \\
$\log (300-S M R 00)$ & 0.526 & $27.6 \%$ \\
$\log (300-S M R 06)$ & 0.449 & $20.2 \%$ \\
$\log (300-S M R 12)$ & 0.554 & $30.7 \%$ \\
$\log (300-S M R 18)$ & 0.627 & $39.3 \%$ \\
$\log \left(1-D_{S T, n e g} *\right)$ & 0.578 & $33.4 \%$ \\
$\log \left(1-D c x_{\text {neg }} *\right)$ & 0.589 & $34.7 \%$ \\
$\log \left(1-D c x_{n e g} *\right)$ & 0.634 & $40.2 \%$ \\
$\log \left(100-D_{S T} *\right)$ & 0.662 & $43.8 \%$ \\
$\log \left(100-D_{c x} *\right)$ & 0.670 & $44.9 \%$ \\
$\log \left(100-D c x_{A} *\right)$ & 0.691 & $47.7 \%$ \\
\hline
\end{tabular}

Table 4.1: The respective cross-correlation coefficient magnitudes and percent of variation explained for ABI in the years 2000 through 2009 inclusive. The indices were processed appropriately to ensure that all logarithms receive positive input. In the case of storm-time magnetic depression, those with a "neg" subscript were set to zero when a positive index value was encountered. Note that pressure corrected Dcx* approaches the explanatory capability of of the K-family indices for ABI, as shown by highlighted examples. The "A" subscript indicates that the pressure correction found for Dcx by Asikainen et al. (2010) was used. 


\begin{tabular}{|c|cc|}
\hline \hline & $|R|$ & $R^{2}$ \\
\hline $\log (1+a p)$ & 0.876 & $76.8 \%$ \\
$\log (1+a m)$ & 0.877 & $76.9 \%$ \\
$\log (1+\alpha 15)$ & 0.841 & $70.6 \%$ \\
$\log \left(1-D_{S T, \text { neg }}\right)$ & 0.960 & $92.1 \%$ \\
$\log \left(1-D c x_{\text {neg }}\right)$ & 0.986 & $97.3 \%$ \\
$\log \left(1-D_{S T, \text { neg }} *\right)$ & 0.989 & $97.7 \%$ \\
$\log \left(1-D c x_{\text {neg }} *\right)$ & 0.996 & $99.2 \%$ \\
$\log \left(1-D c x_{A, \text { neg }} *\right)$ & 0.996 & $99.1 \%$ \\
\hline
\end{tabular}

Table 4.2: The respective cross-correlation coefficient magnitudes and percent of variation explained for $2^{o}$ binned ABI by median values of the index logarithms shown the years 2000 through 2009 inclusive. The indices were processed appropriately to ensure that all logarithms receive positive input. In the case of storm-time magnetic depression, index values were set to zero when a positive index value was encountered. Note that k-family indices have lower correlations with ABI than storm depression indices, with pressure corrected Dcx* exceeding the $99 \%$ level, as shown by highlighted examples. The "A" subscript indicates that the pressure correction found for Dcx by Asikainen et al. (2010) was used. 


\section{Figures}




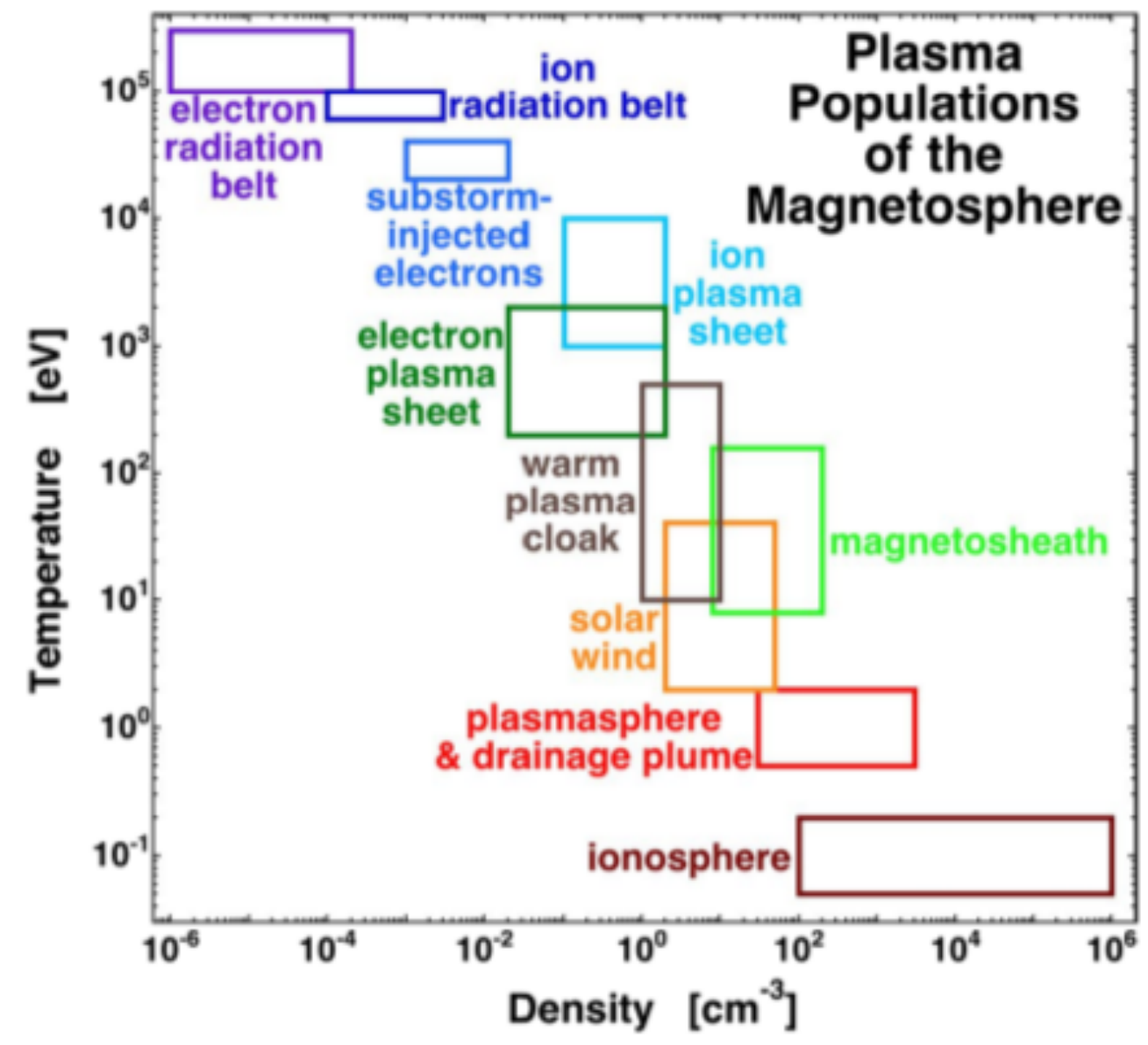

Figure 1.1: Typical plasma parameters for the solar wind in Earth's vicinity are shown along with those associated with other plasma regions of geospace. From Borovsky and Valdivia (2018). 


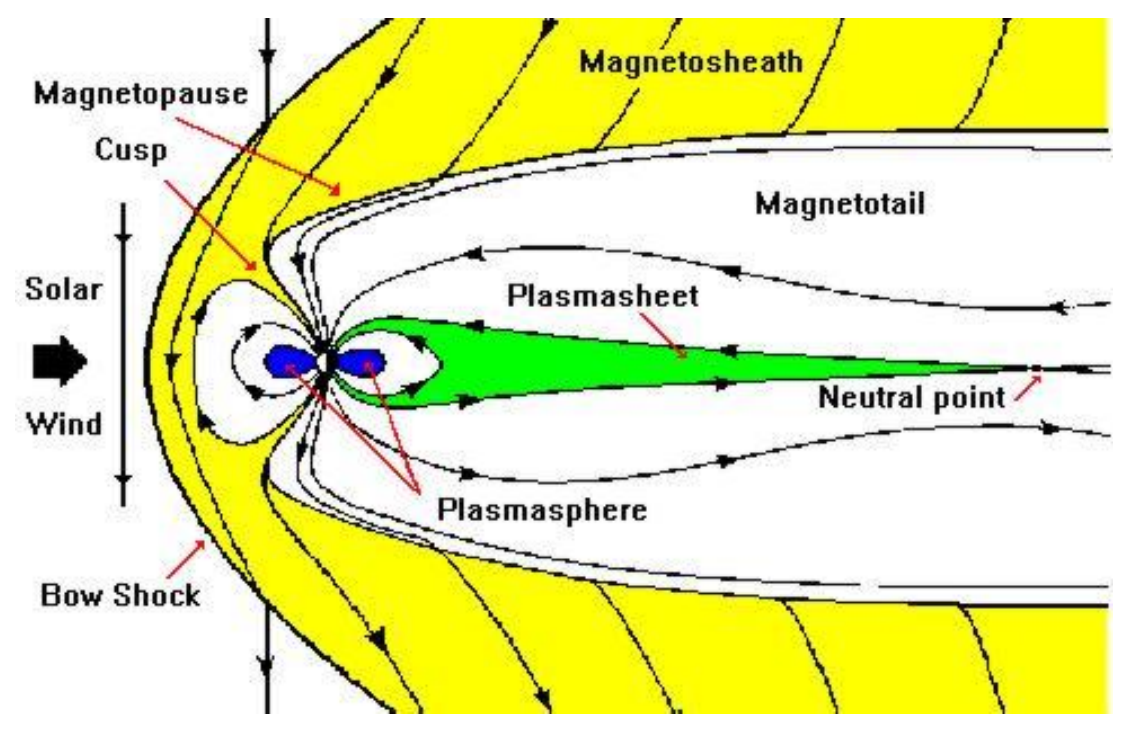

Figure 1.2: The magnetosphere is here shown in cross-section. 


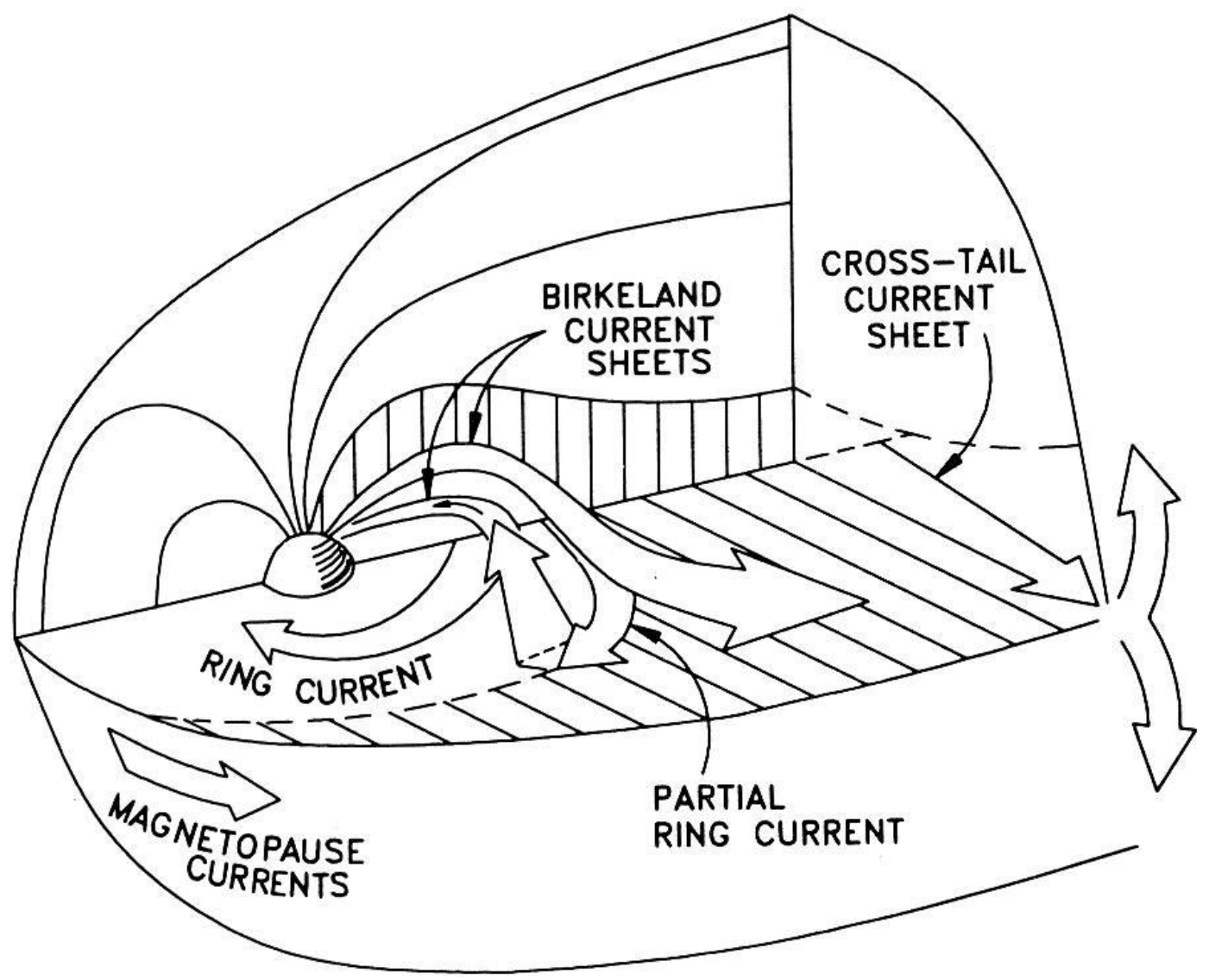

Figure 1.3: The magnetosphere is here shown with major current systems labeled. 


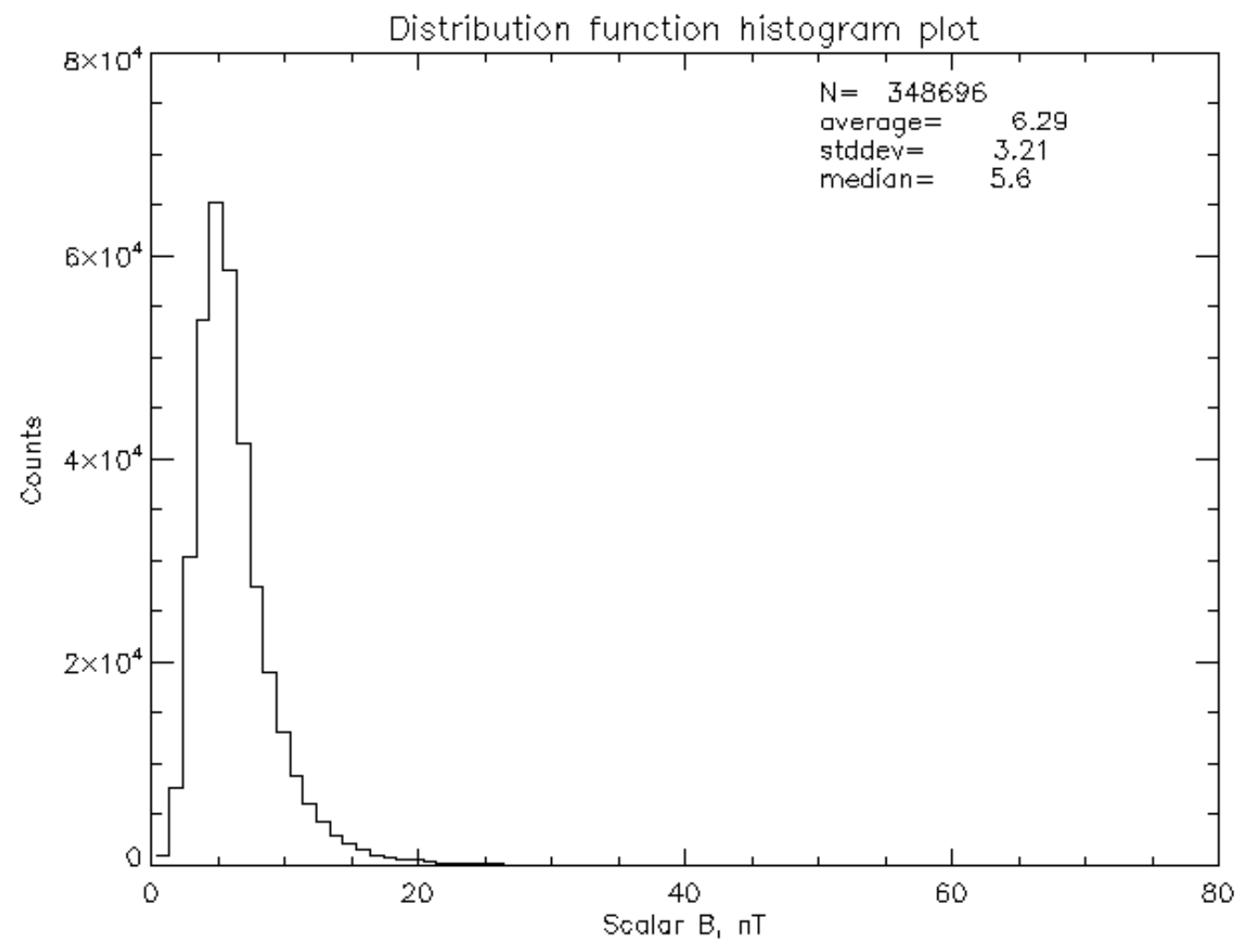

Figure 1.4: The SW magnetic field magnitude distribution and statistics for the years 1965 through 2016. Obtained from omniweb.gsfc.nasa.gov. 


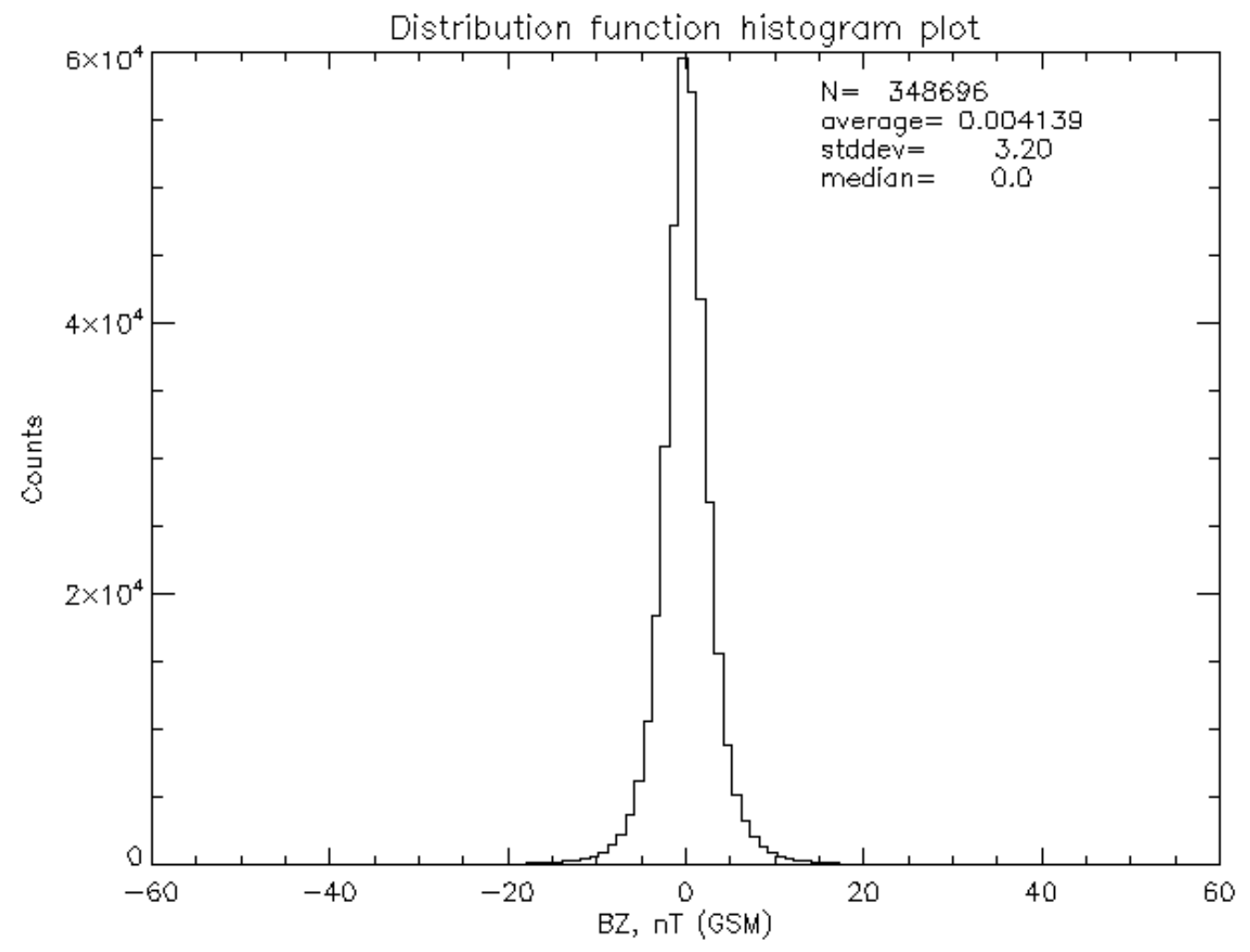

Figure 1.5: The SW magnetic field GSM z-component (parallel to Earths magnetic dipole axis) distribution and statistics for the years 1965 through 2016. Obtained from omniweb.gsfc.nasa.gov. 


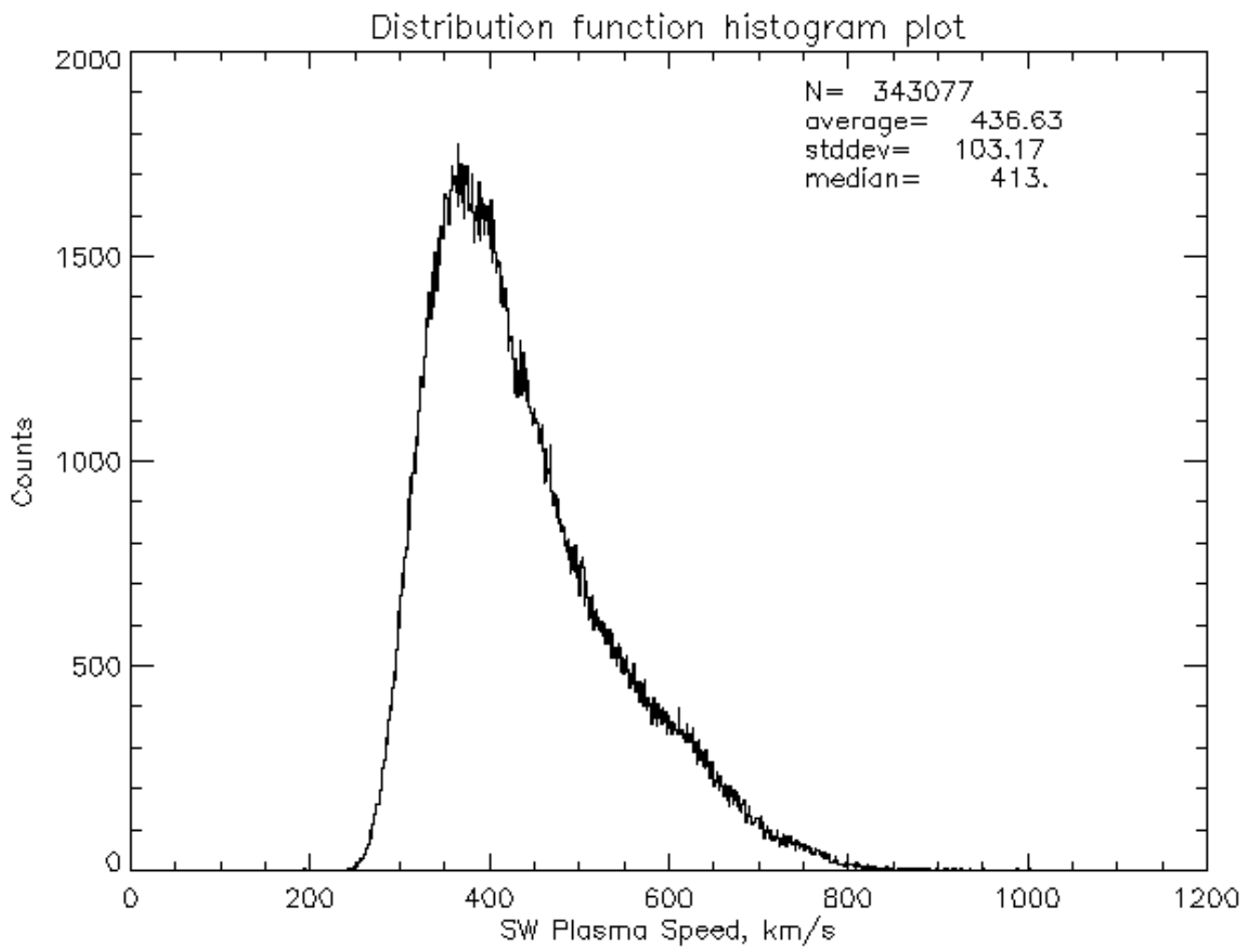

Figure 1.6: The SW speed distribution and statistics for the years 1965 through 2016. Obtained from omniweb.gsfc.nasa.gov. 


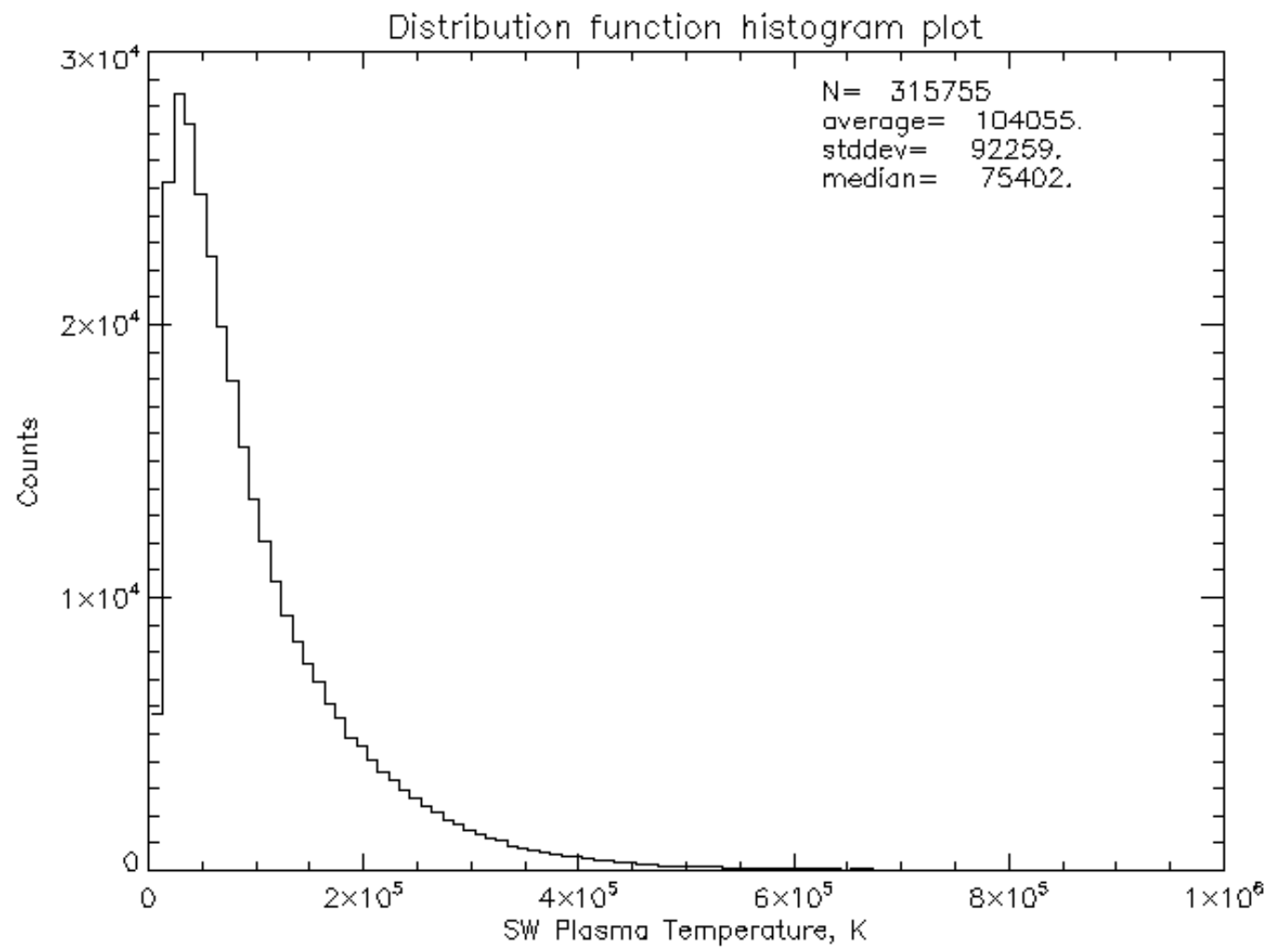

Figure 1.7: The SW proton temperature distribution and statistics for the years 1965 through 2016. Obtained from omniweb.gsfc.nasa.gov. 


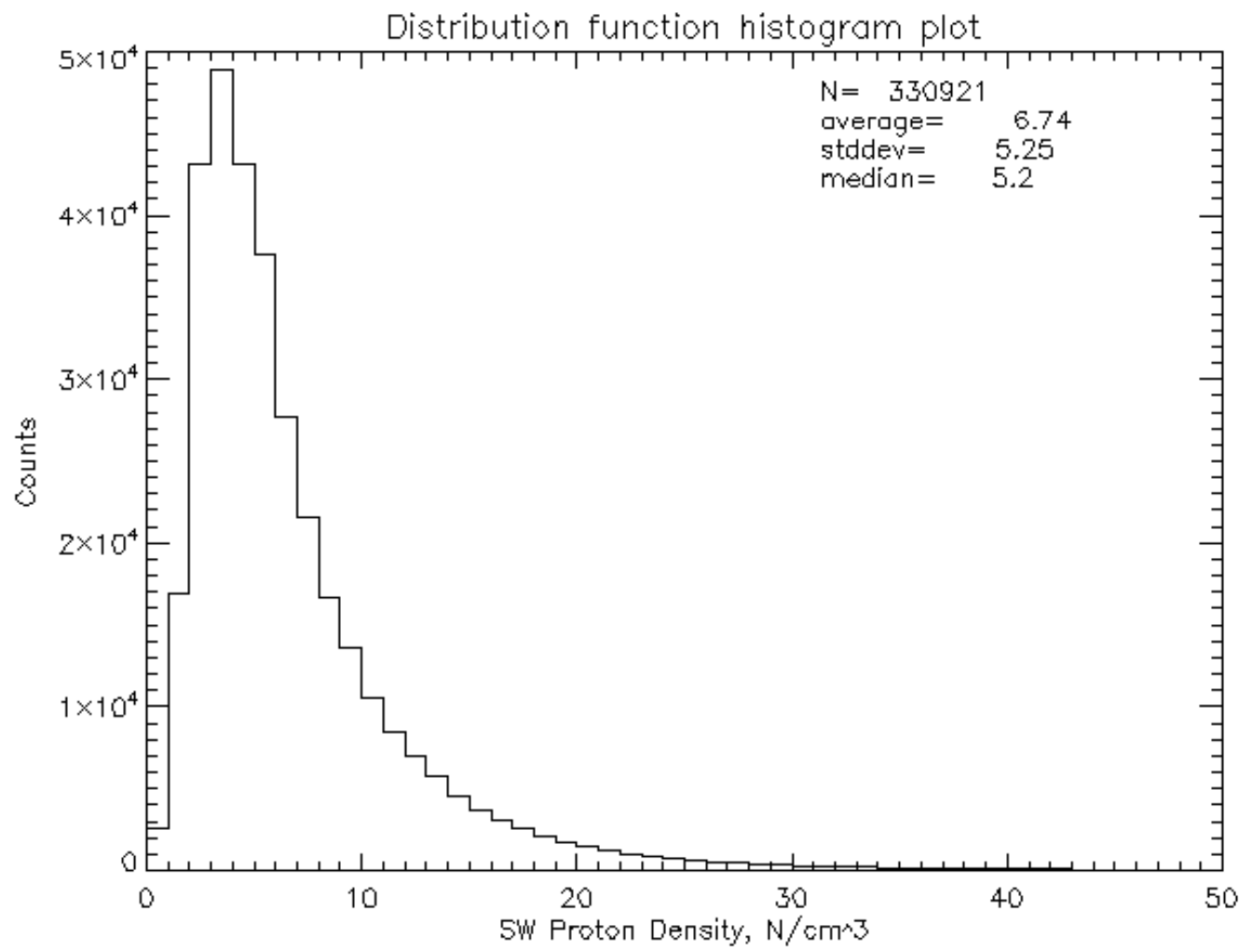

Figure 1.8: The SW density distribution and statistics for the years 1965 through 2016. Obtained from omniweb.gsfc.nasa.gov. 


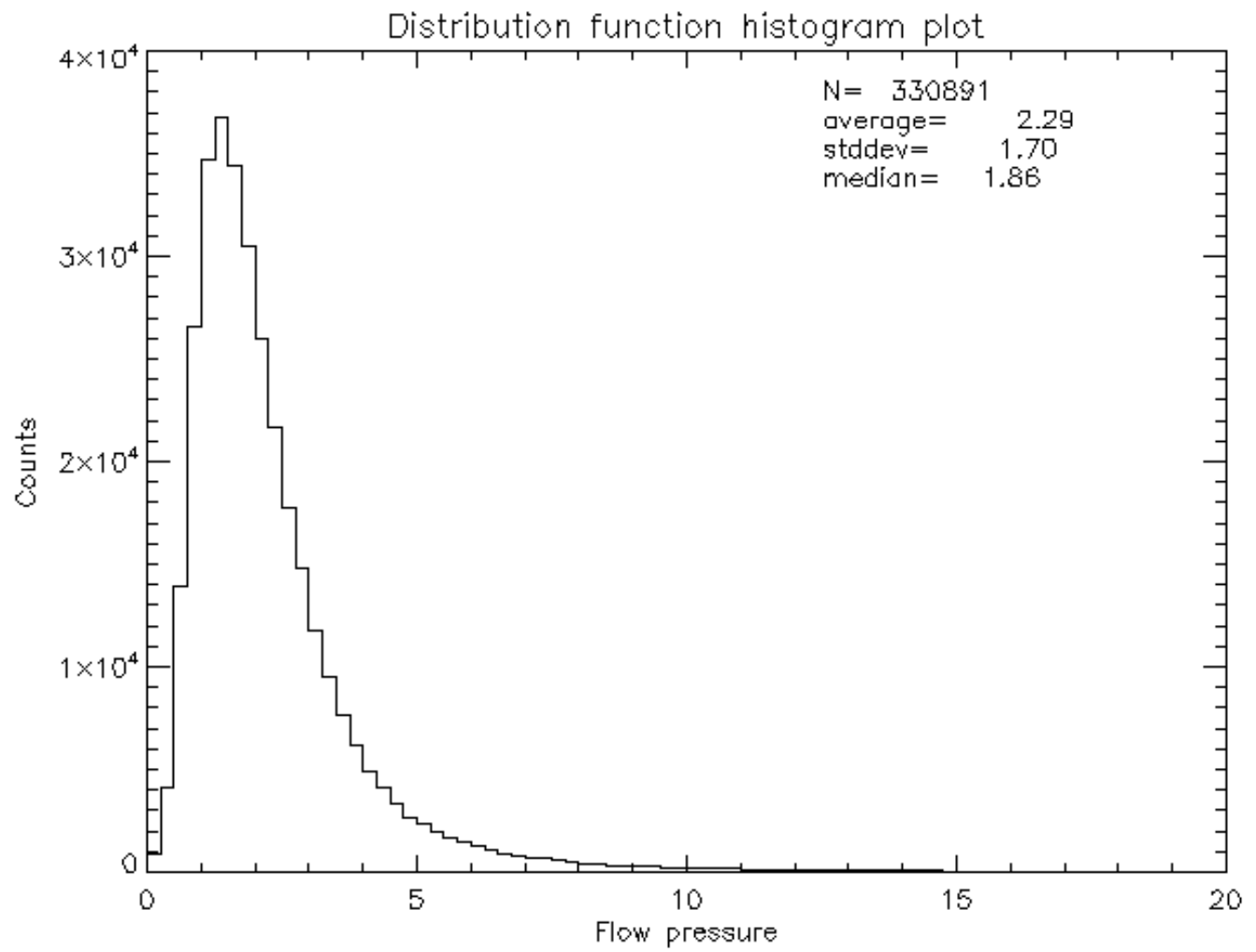

Figure 1.9: The SW dynamic (ram) pressure distribution and statistics for the years 1965 through 2016. Obtained from omniweb.gsfc.nasa.gov. 


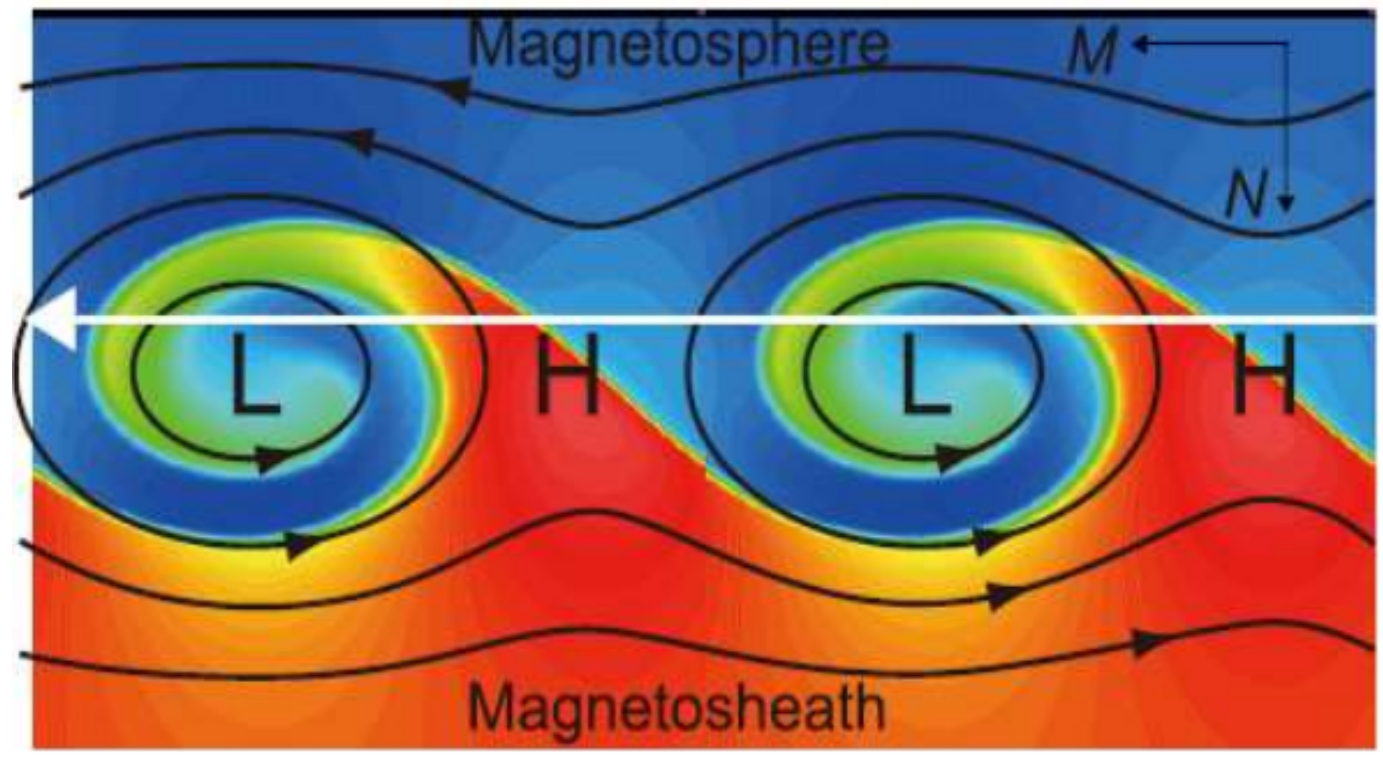

Figure 1.10: A schematic drawing of rolled-up Kelvin-Helmholtz vortices at the dusk-flank magnetopause is shown. The streamline pattern (black lines) and total (magnetic plus plasma) pressure and density (red, dense; blue, tenuous) distributions, when viewed in the vortex rest frame. The sub-solar region is to the left. From supplementary figure 4 of Kavosi and Raeder (2015). 


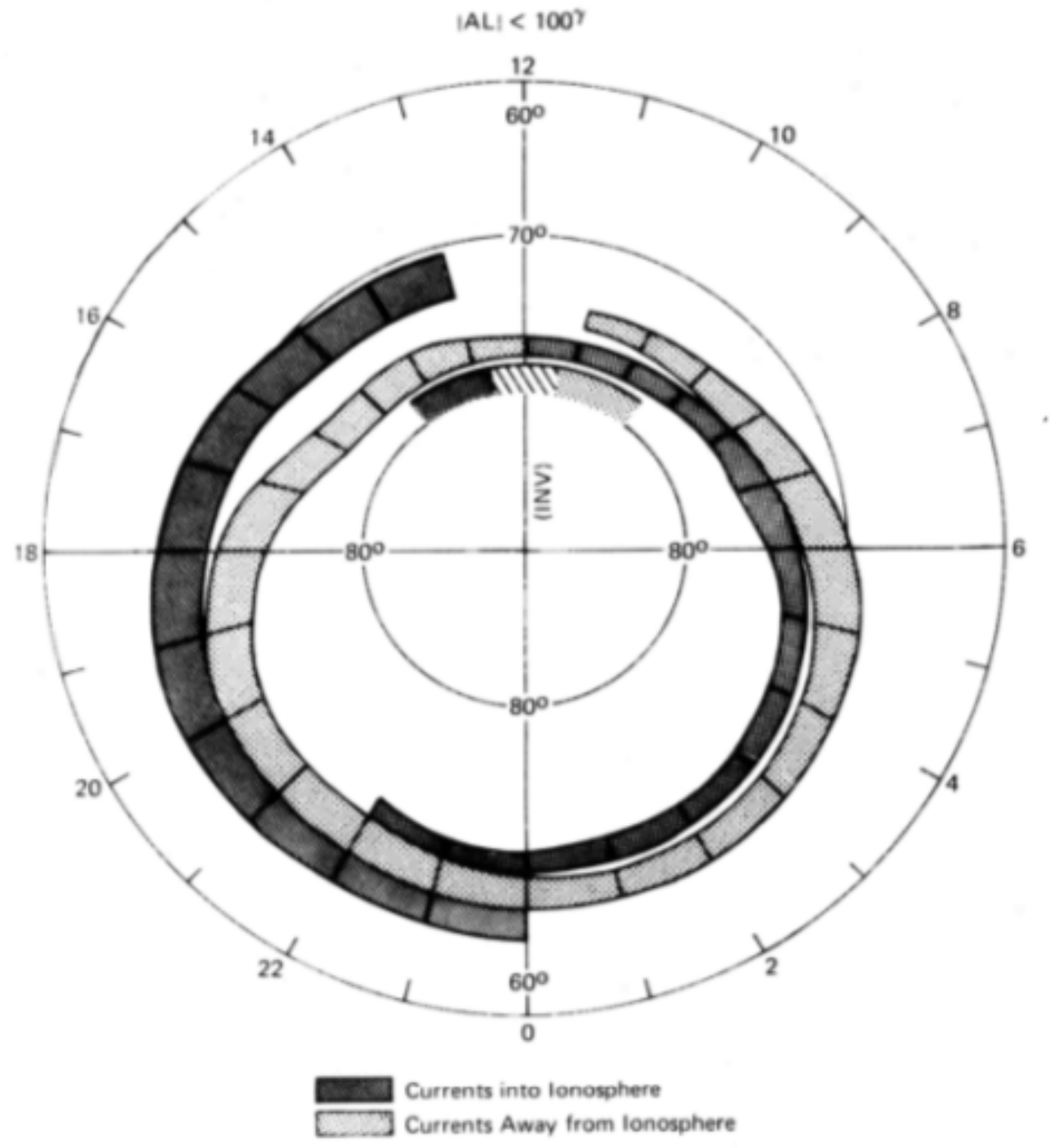

Figure 1.11: The typical arangement of field aligned currents for relatively undisturbed times $(A L<100 n T)$. The R0 currents are the small system of currents near the pole. The R1 currents are the inner (poleward) ring of currents. The $\mathrm{R} 2$ currents are the outer (equaterward) ring of currents. The center is $90^{\circ}$ magnetic latitude (i.e. the northern magnetic pole). The outer edge is the $60^{\circ}$ magnetic latitude. The numbers around the outer edge specify magnetic local time (MLT). From Iijima and Potemra (1976). 


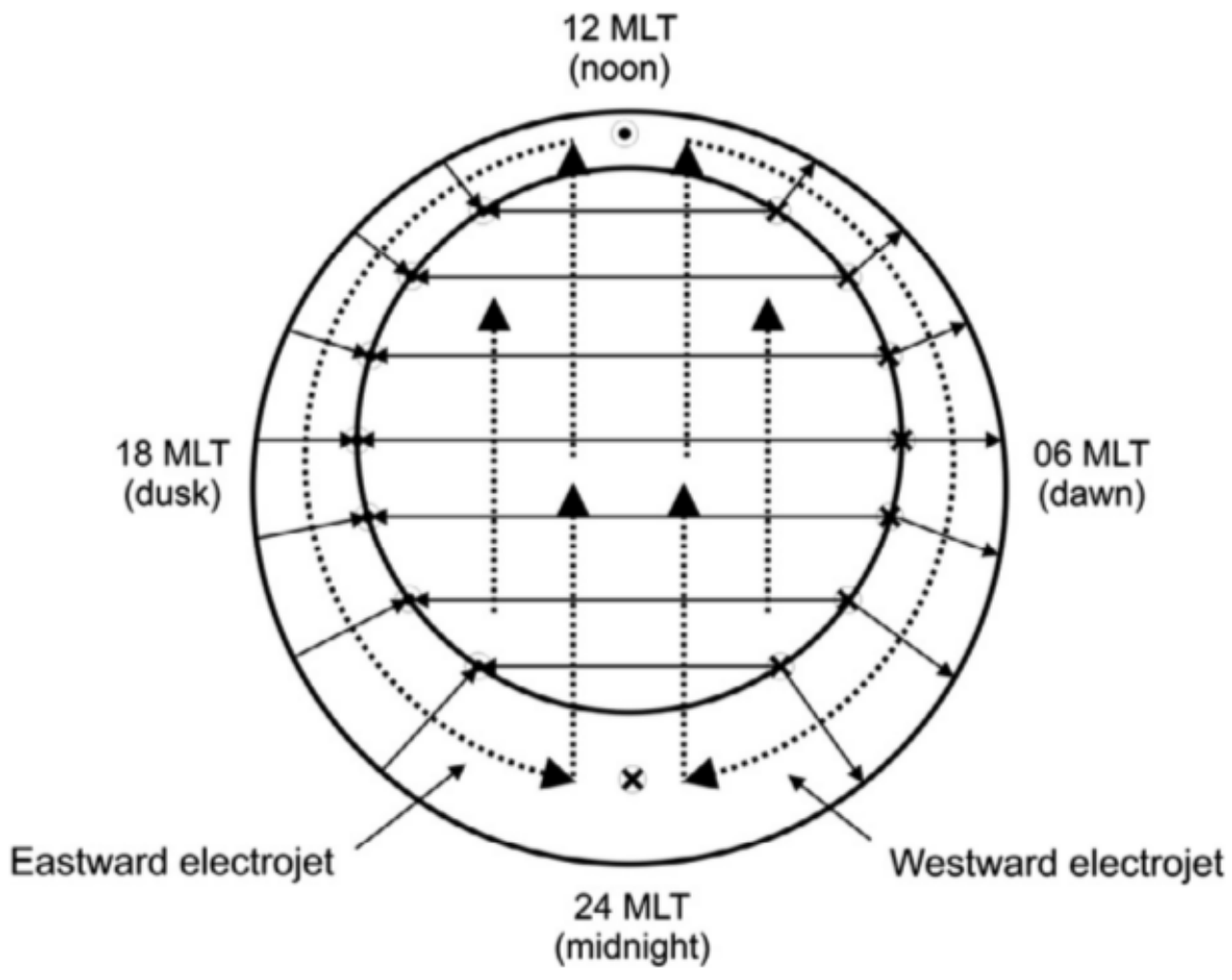

Figure 1.12: A simplified schematic of the auroral electrojet currents in the auroral oval. Hall currents are represented by dotted arrows and electric fields by solid arrows. From figure 2 in Johnsen (2013). 


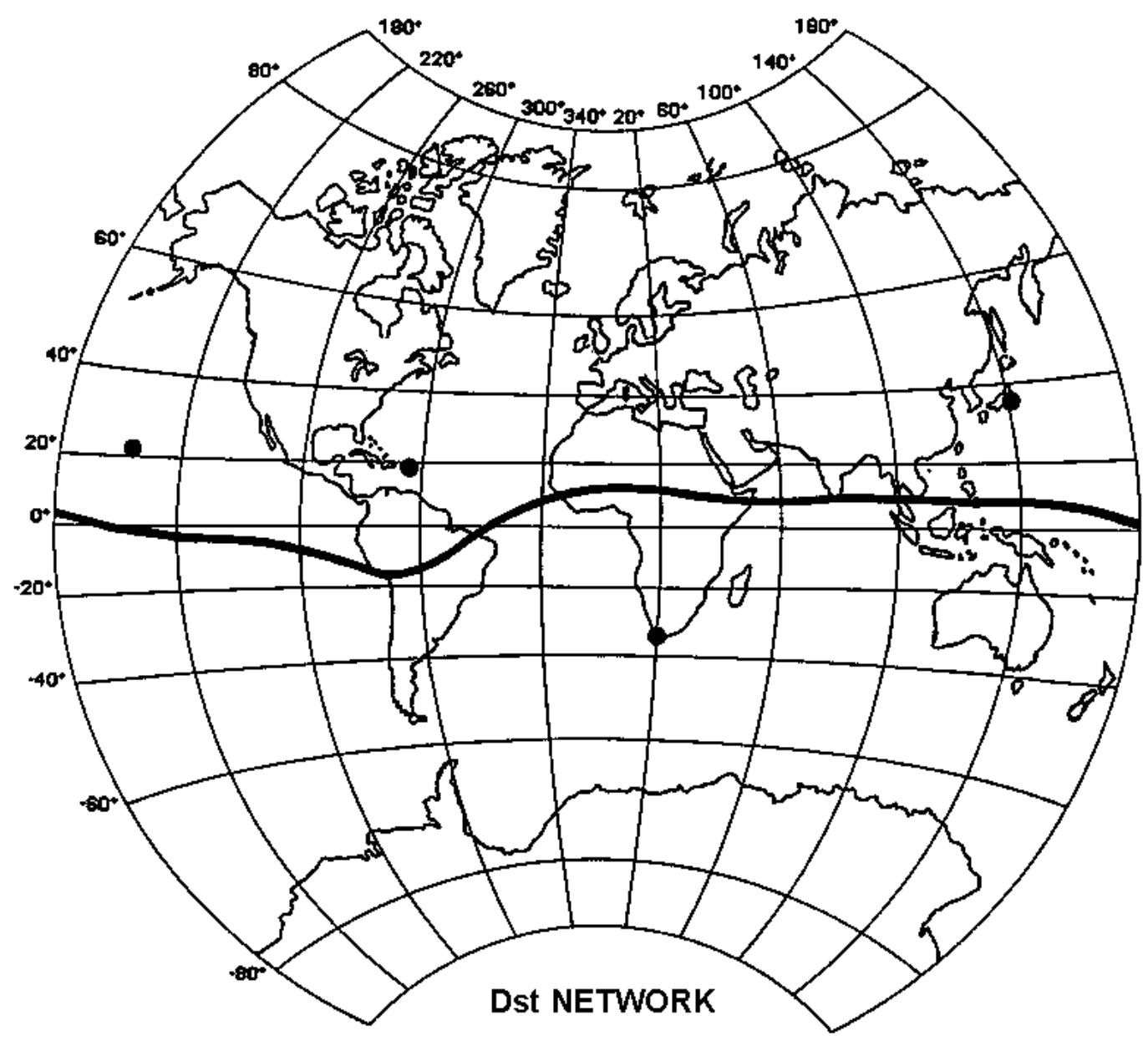

Figure 1.13: The locations of magnetometers used in the calculation of $D_{S T}$ are shown. The bold line represents the magnetic equator. From (http://wdc.kugi.kyoto-u.ac.jp). 


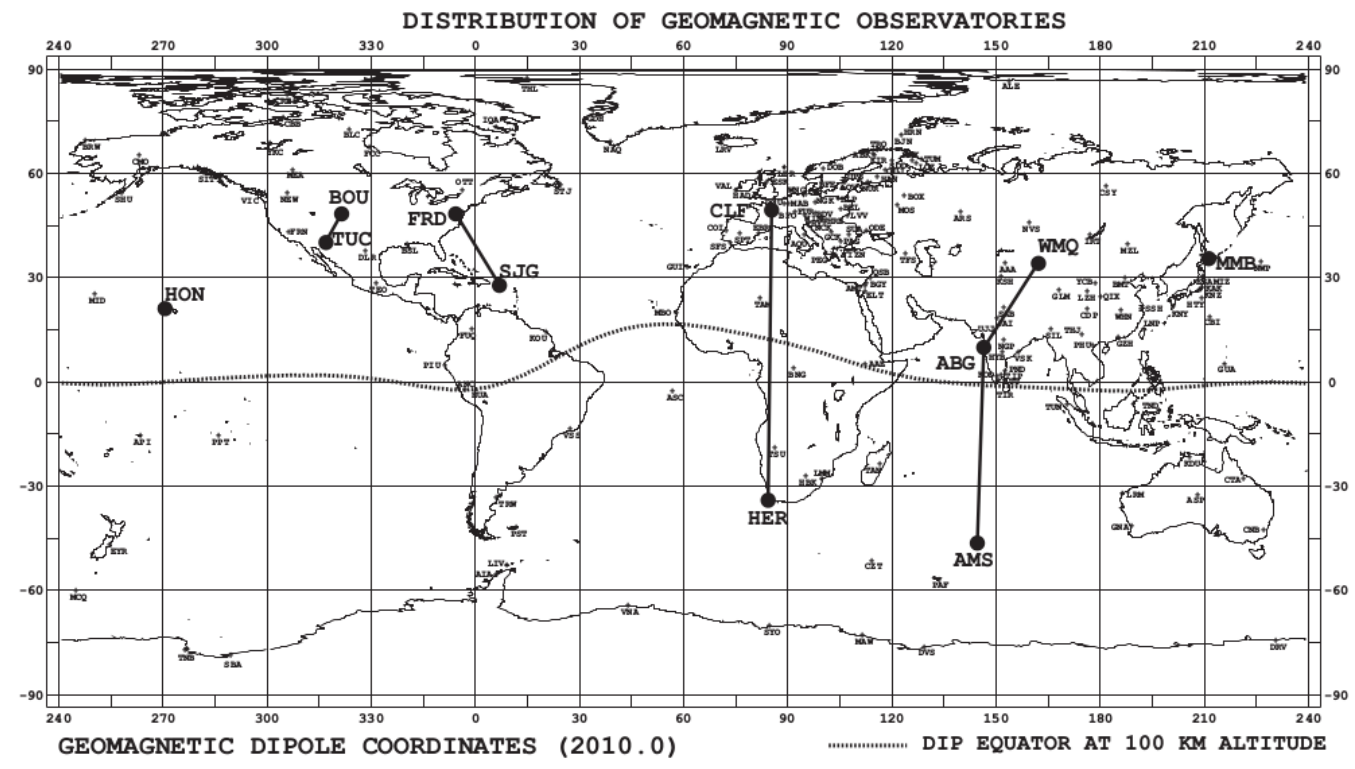

Figure 1.14: The locations of magnetometers used in the calculation of Sym/H and ASY/H are shown. The dotted line represents the magnetic equator. Those stations connected by solid lines represent alternate choices used in index calculations according to availability and quality of data. From (http://wdc.kugi.kyoto-u.ac.jp). 


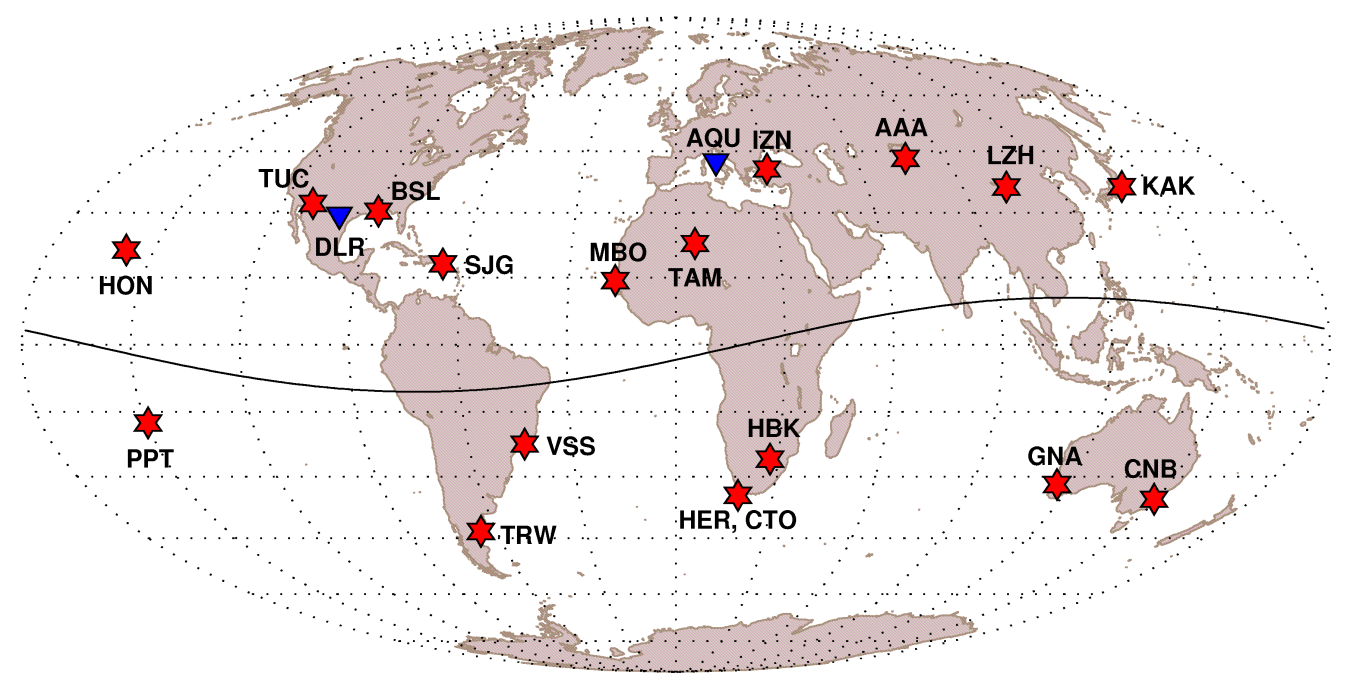

Figure 1.15: The locations of magnetometers used in the calculation of Dcx are shown. The curved line represents the magnetic equator. From (http://dcx.oulu.fi/?link=stations). 


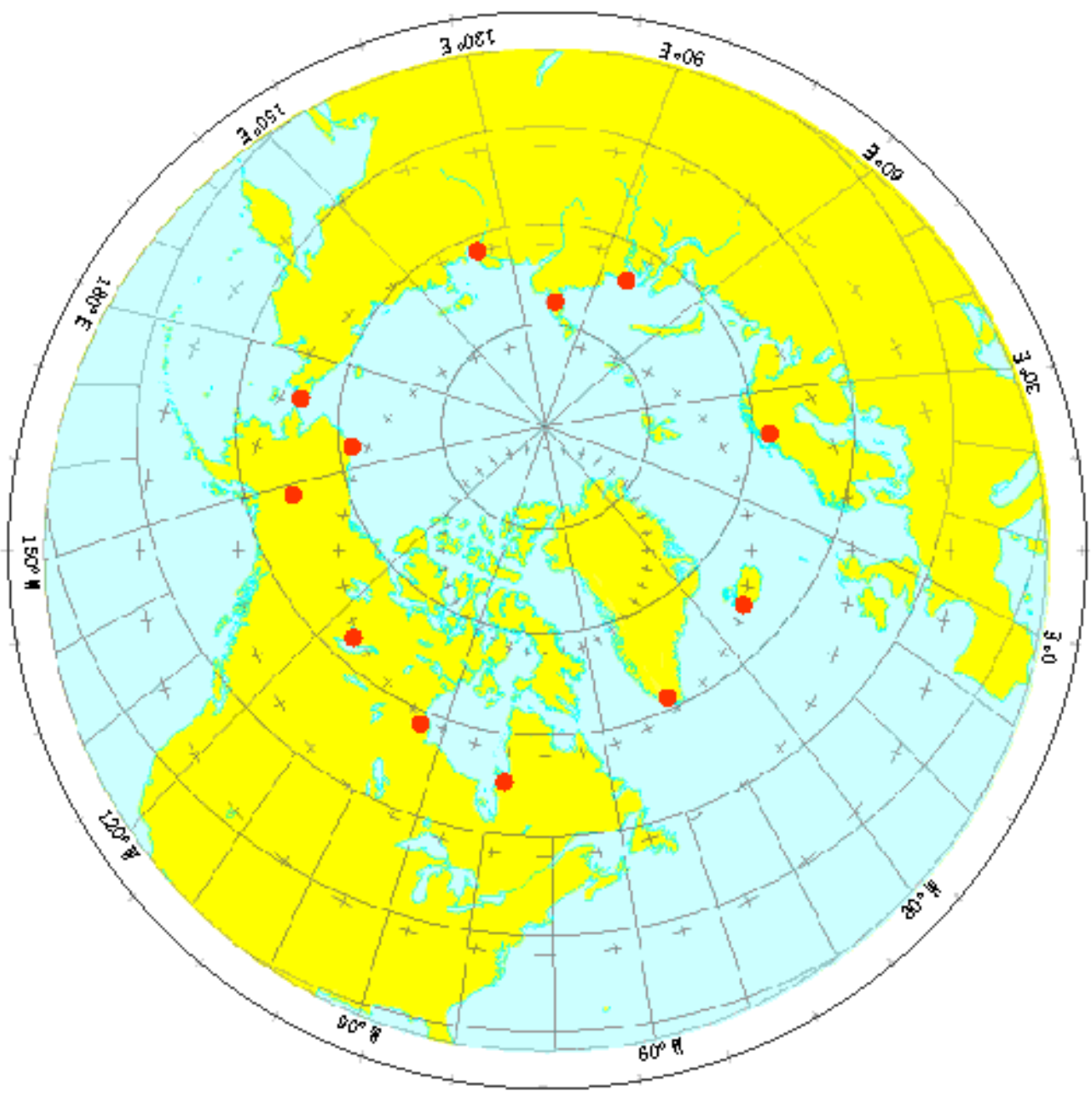

Figure 1.16: The locations of magnetometers used in the calculation of AL, AU, and AE are shown. Plus signs represent magnetic coordinates. From (http://wdc.kugi.kyoto-u.ac.jps). 


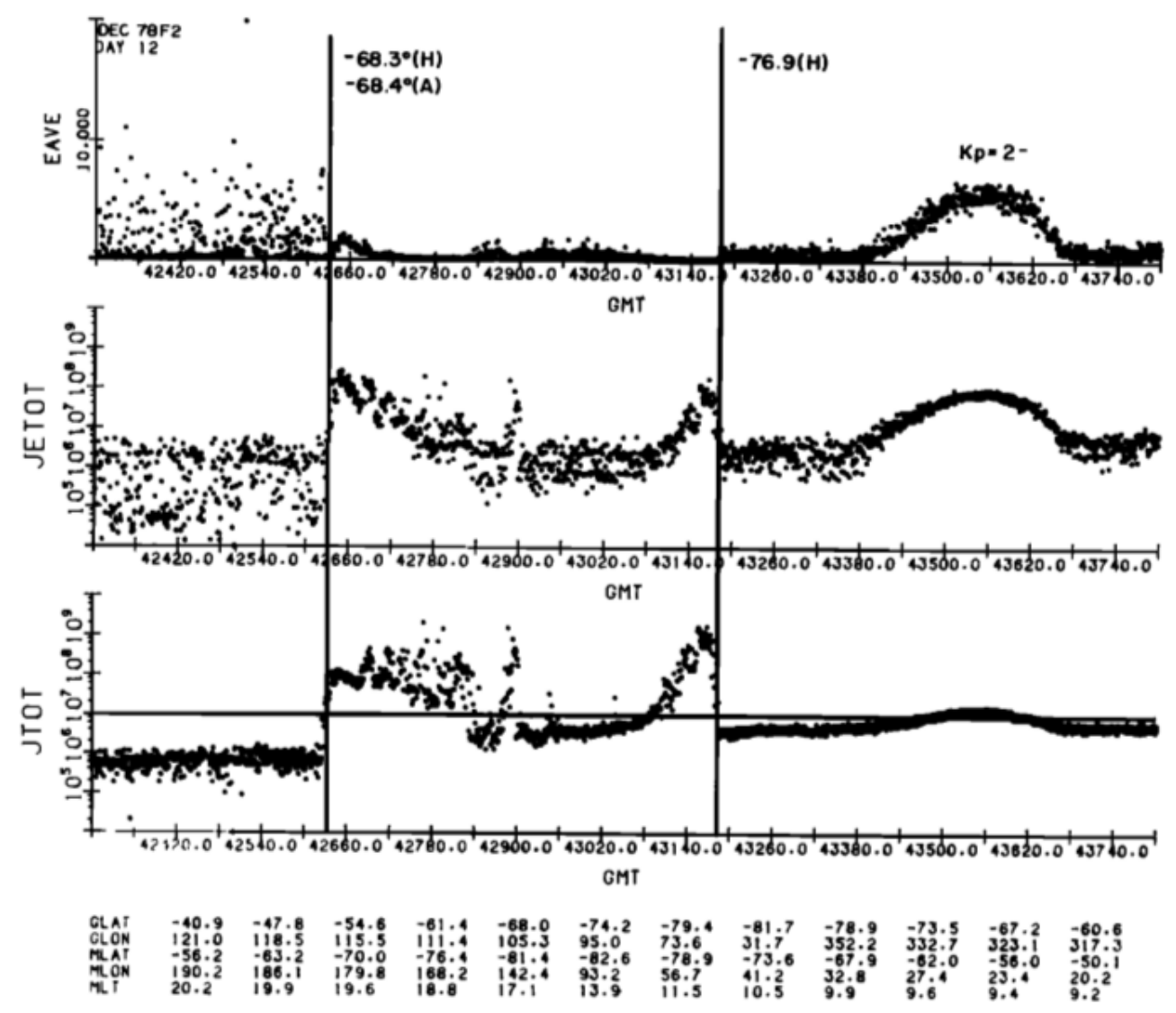

Figure 1.17: An example of DMSP sourced electron precipitation data, from a pass over the Southern pole on Dec. 12, 1978, used to produce ABI, taken from Gussenhoven et al. (1983), is shown with precipitation boundaries used marked by solid vertical lines. Electon flux, in $\left(\mathrm{cm}^{2} s s r\right)^{-1}$, is on the bottom. Energy, in $\mathrm{KeV} /\left(\mathrm{cm}^{2} \mathrm{srs}\right)$, is shown in the middle. The top pannel shows average electron energy in $\mathrm{KeV}$. 

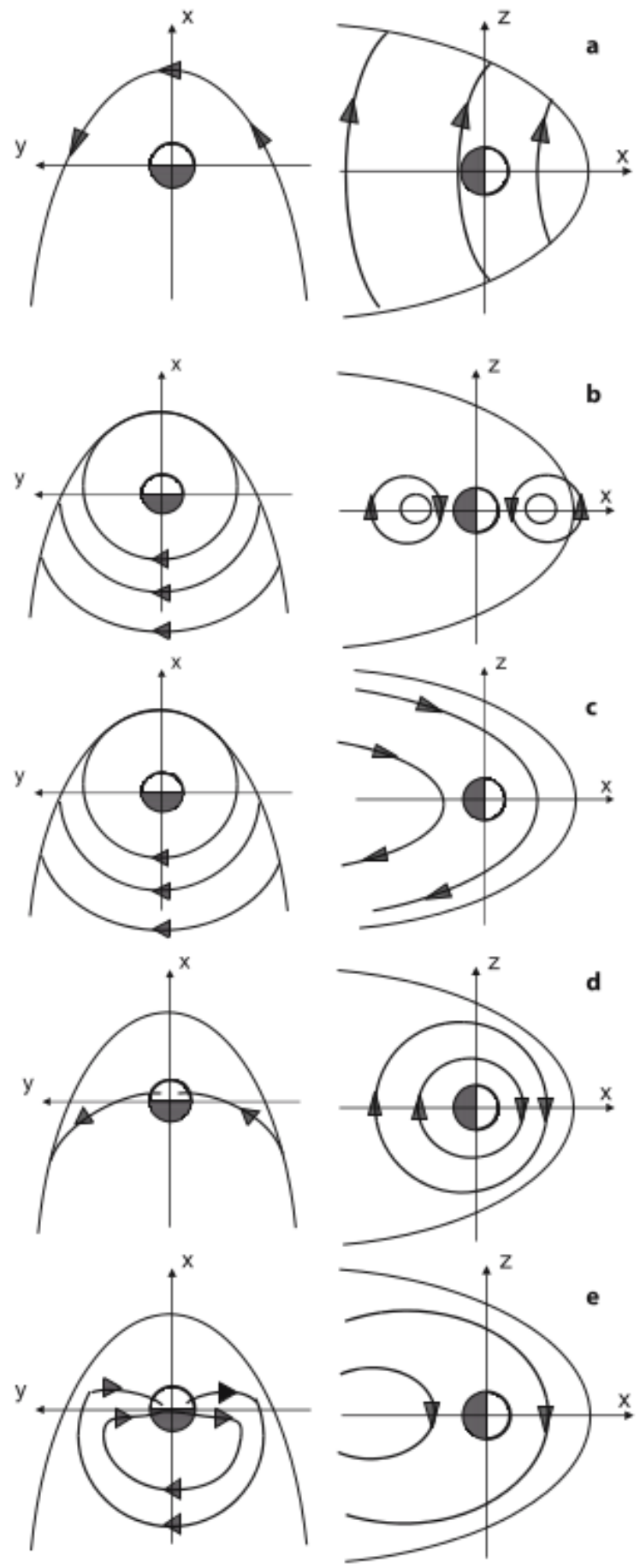

Figure 1.18: The magnetic influences of different magnetospheric current systems are shown, with electric currents and magnetic fields represented by arrows on diagrams in the left-hand and righthand columns, respectively. (a) shows the effect of magnetopause currents. (b) shows the effect of symmetric ring currents. (c) shows the effect of cross-tail current. (d) shows the effect of R1 FAC. (e) shows the effect of partial ring currents their R2 FAC closure. From Kamide and Maltsev (2007). 


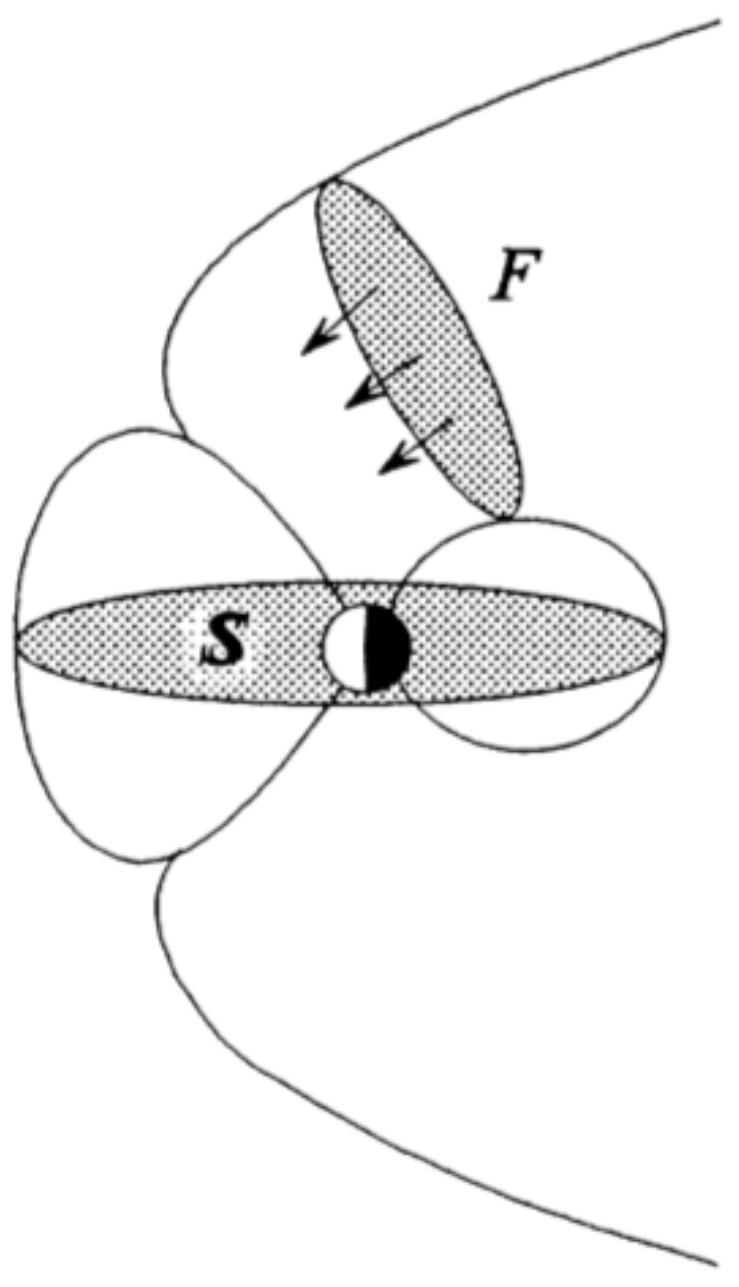

Figure 1.19: The flux in the inner magnetosphere, denoted by $S$, and the outer magnetosphere, denoted by $F$ used by to calculate the approximate effect of cross-tail currents upon storm-time magnetic depression by Maltsev and his collaborators, is shown. From Maltsev (1996). 


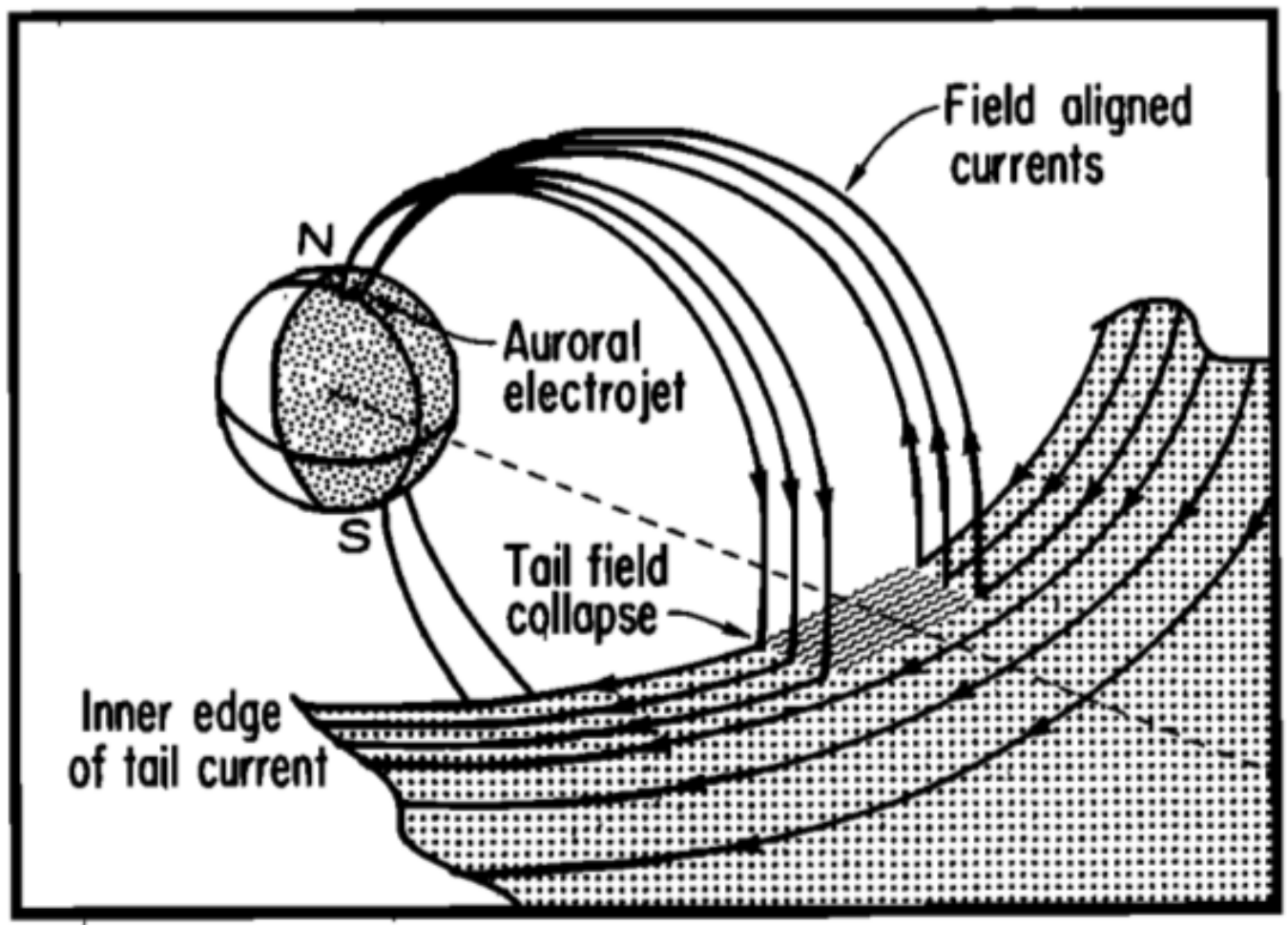

Figure 1.20: This diagram shows the general pattern of the substorm current wedge upon the disruption of the tail current sheet during a substorm. From McPherron et al. (1973). 


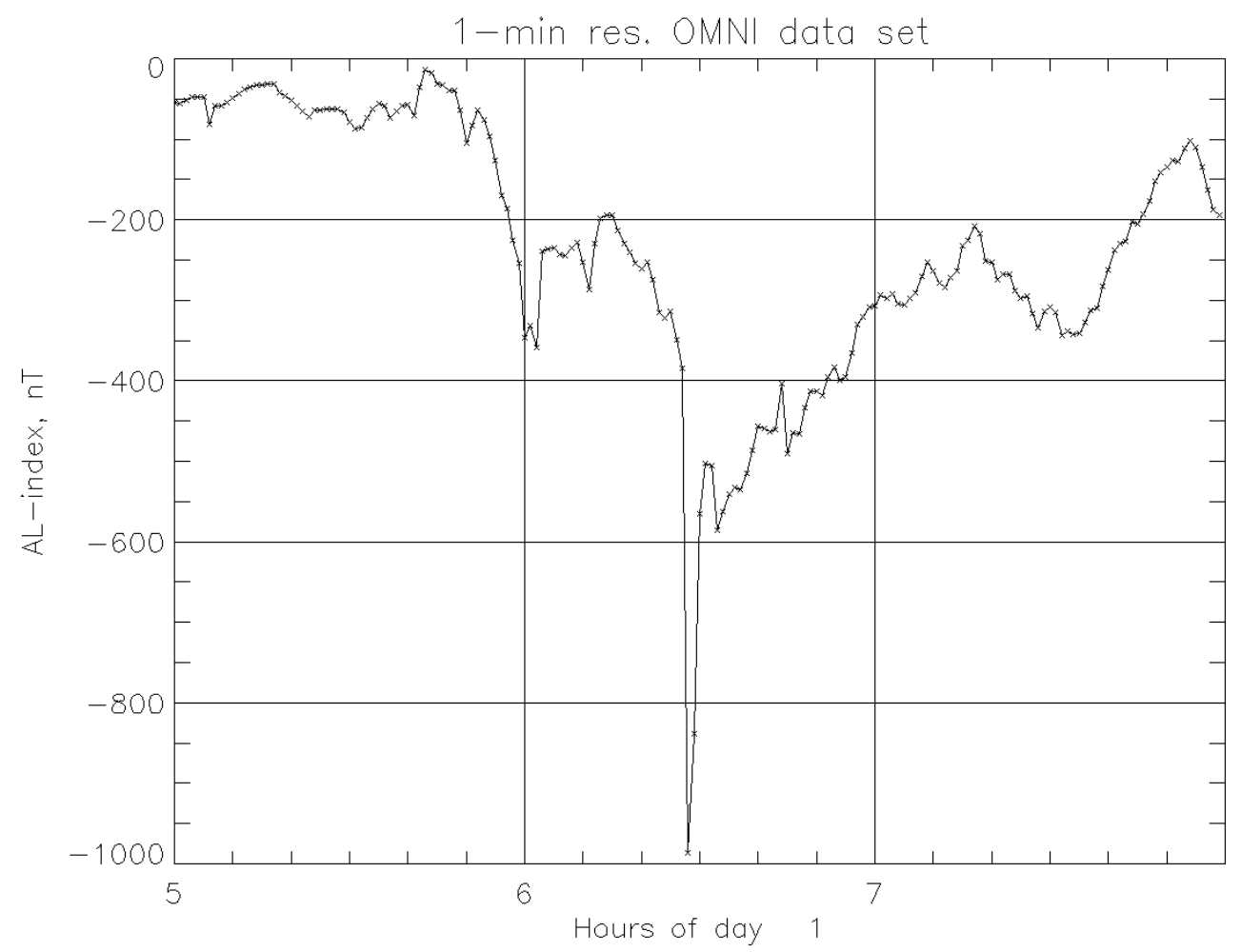

Figure 1.21: An example of the Auroral Lower (AL) index of an interval with substorm activity, produced using high resolution Omni, is shown. The rapid negative excursion of AL during the substorm expansion is clearly visible. 


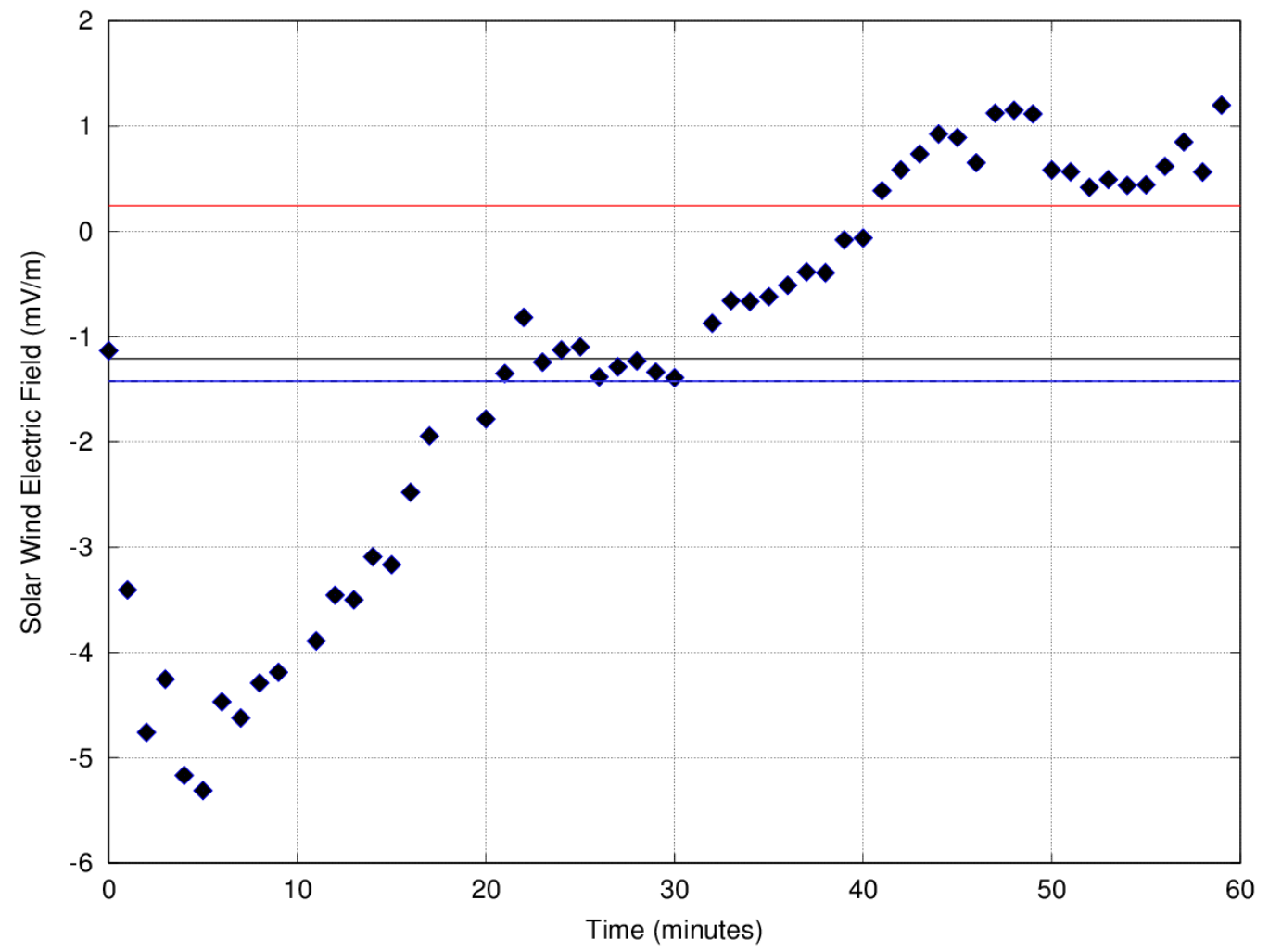

Figure 2.1: An example plot of 1 hour of solar wind electric field, $-v B_{Z}$, taken from the high resolution Omni database, is shown at 1-minute cadence. The black and blue horizontal lines represent the mean values for the hour, from this data and the low resolution Omni database, respectively. One can see that The mean values are negative and would be set to zero if $v B_{S}$ were calculated for the hour. Conversely, the red horizontal line represents the avarage value of rectified $v B_{S}$, showing that energy and flux should transfer from the solar wind during this interval, assiming $v B_{S}$ is a good measure of solar wind magnetosphere energy transfer. 

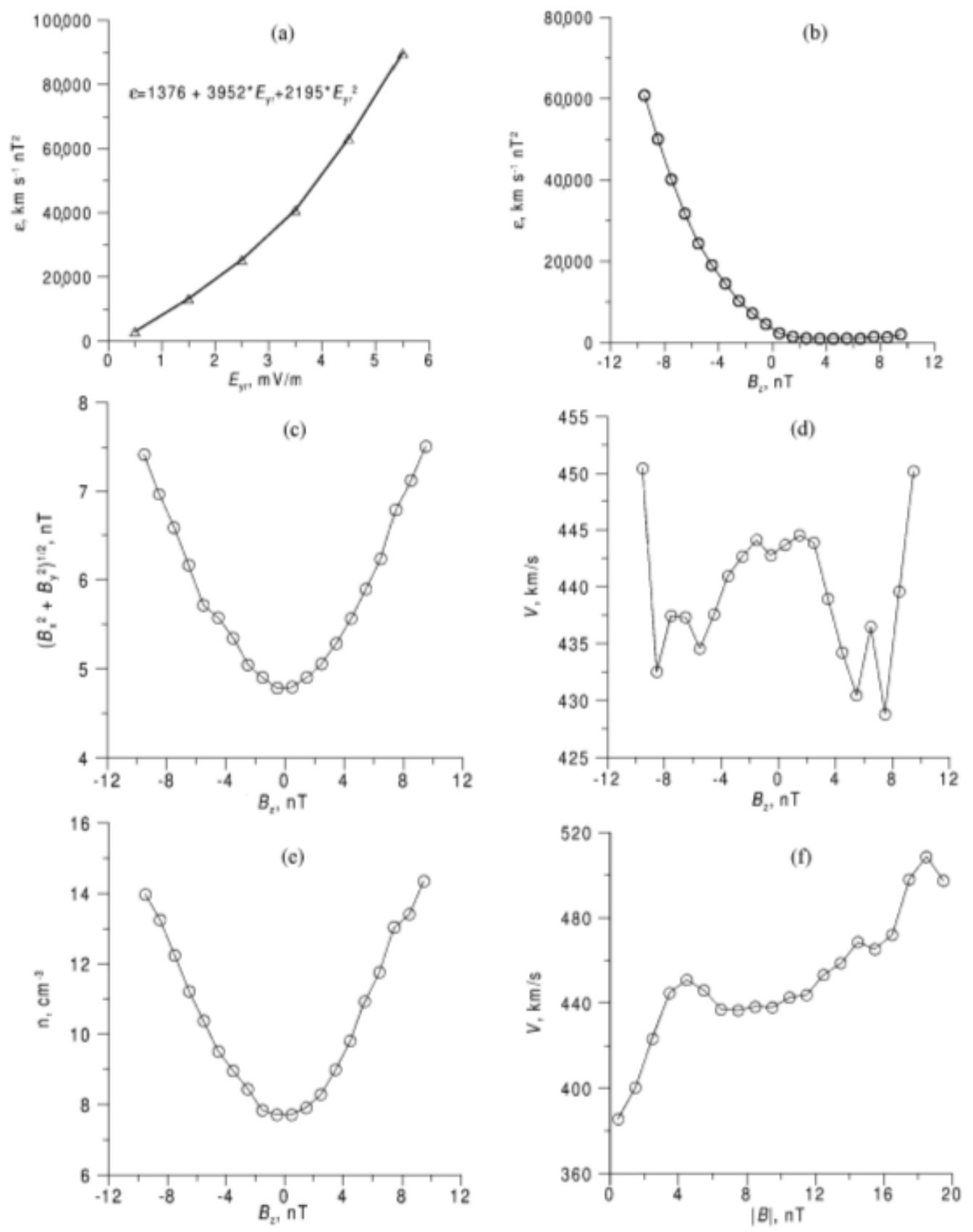

Figure 2.2: Statistical mutual dependencies of solar wind parameters are shown. (a) shows the relation of a widely use $\epsilon$ parameter Perreault and Akasofu (1978) to rectified SW dawn-dusk electric field. (b) shows the relation of $\epsilon$ to IMF $B_{Z}$. (c) shows the relation between IMF magnetic field in the GSM X-Y plane to IMF $B_{Z}$. (d) shows the relation between SW speed and IMF $B_{Z}$. (e) shows the relation between SW density and IMF $B_{Z}$. (f) shows the relation between SW speed and IMF magnitude. From figure 7 in Maltsev and Rezhenov (2003). 

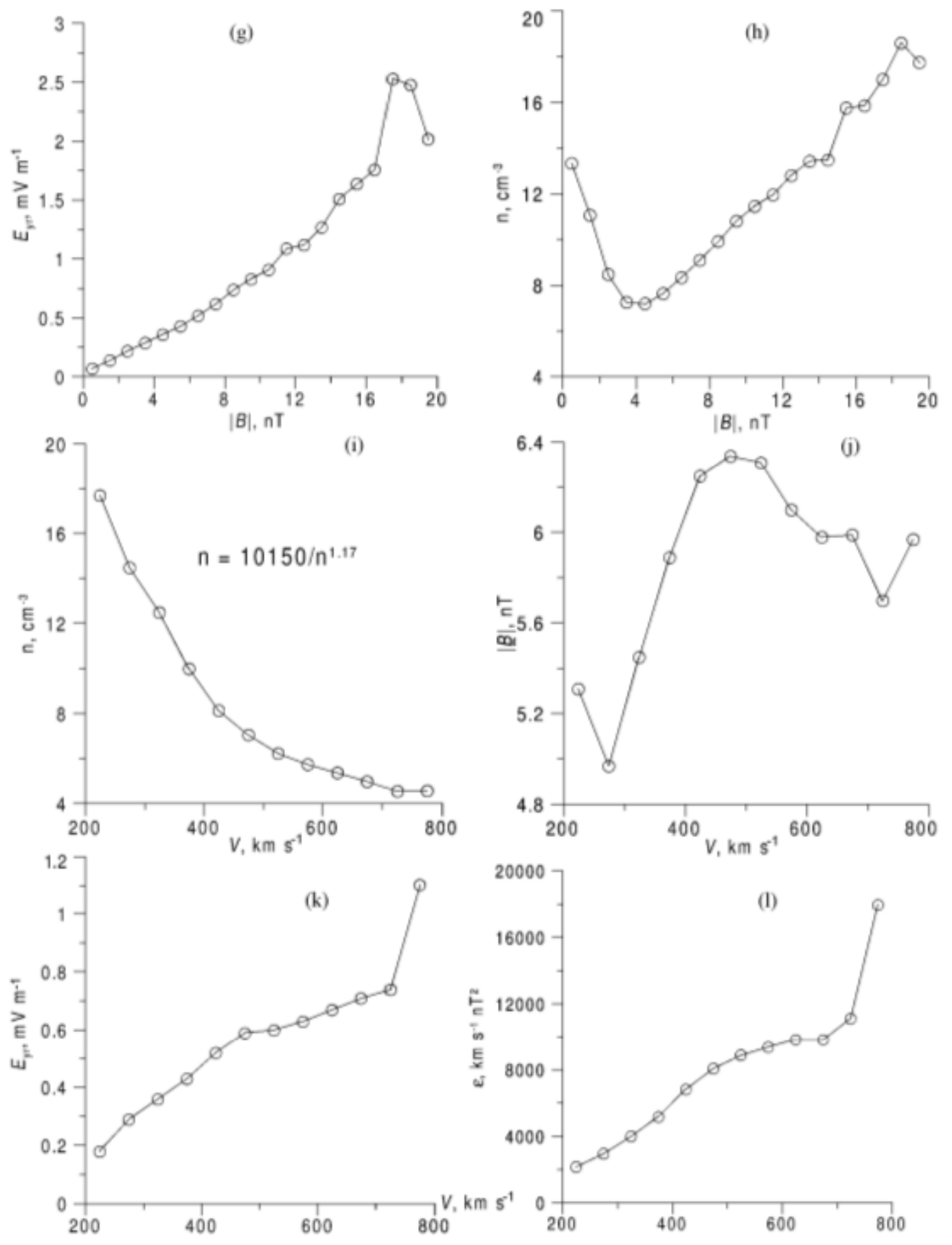

Figure 2.3: Statistical mutual dependencies of solar wind parameters are shown. (g) shows the relation between rectified SW dawn-dusk electric field $\left(v B_{S}\right)$ and IMF $B_{Z}$. (h) shows the relation between SW density and IMF magnitude. (i) shows the relation between SW density and SW flow speed. (j) shows the relation between IMF magnitude and SW flow speed. (k) shows the relation between rectified SW dawn-dusk electric field $\left(v B_{S}\right)$ and $\mathrm{SW}$ flow speed. (l) shows the relation between $\epsilon$ and SW flow speed. From figure 7 in Maltsev and Rezhenov (2003). 


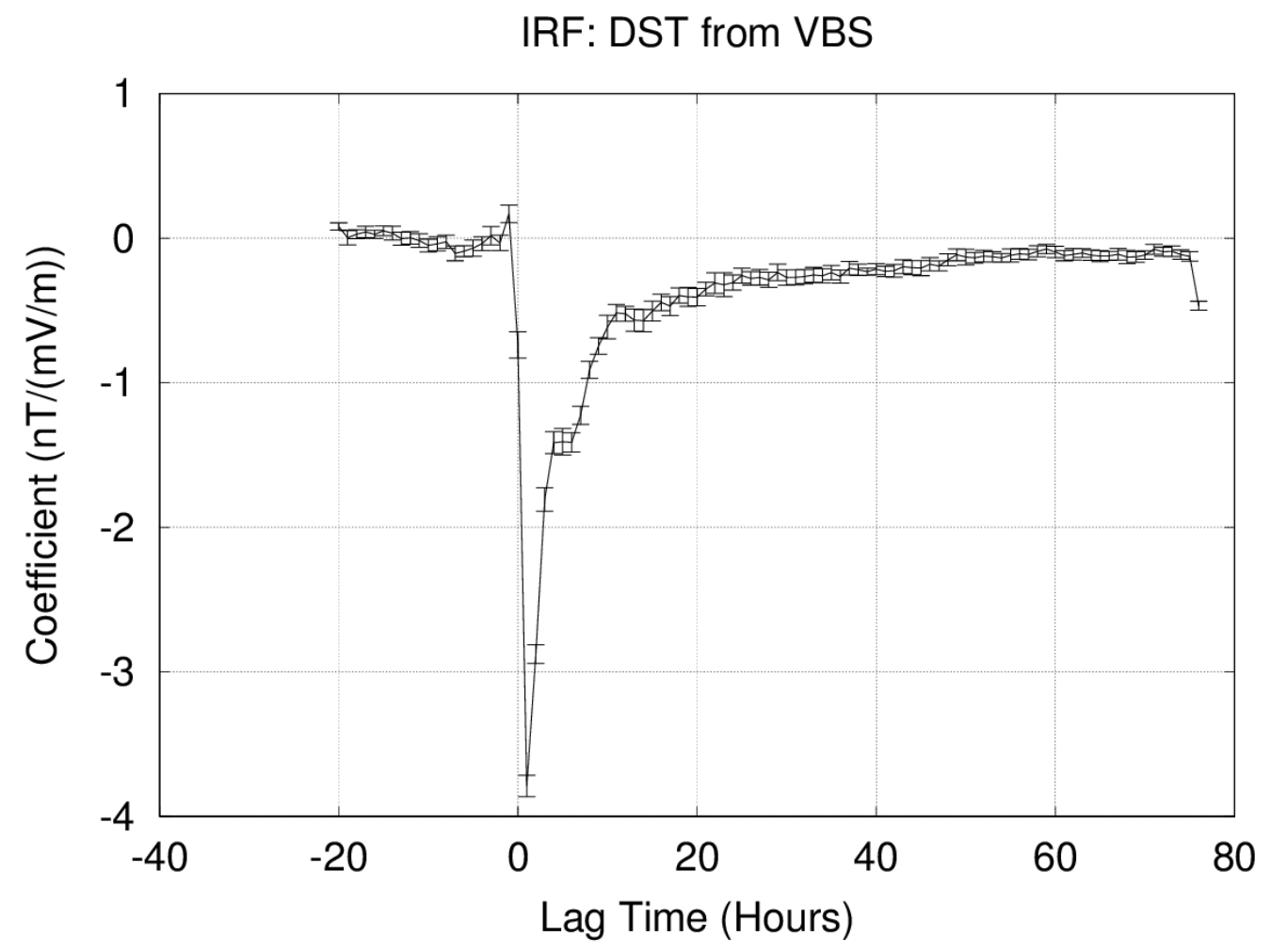

Figure 3.1: The impulse response of DST with respect to vBs. The horizontal axis represents hours of lag time. The vertical axis represents the response of DST (nT) per unit of rectified solar wind electric field $(\mathrm{mV} / \mathrm{m})$. 
IRF: DST from sqrt(P)

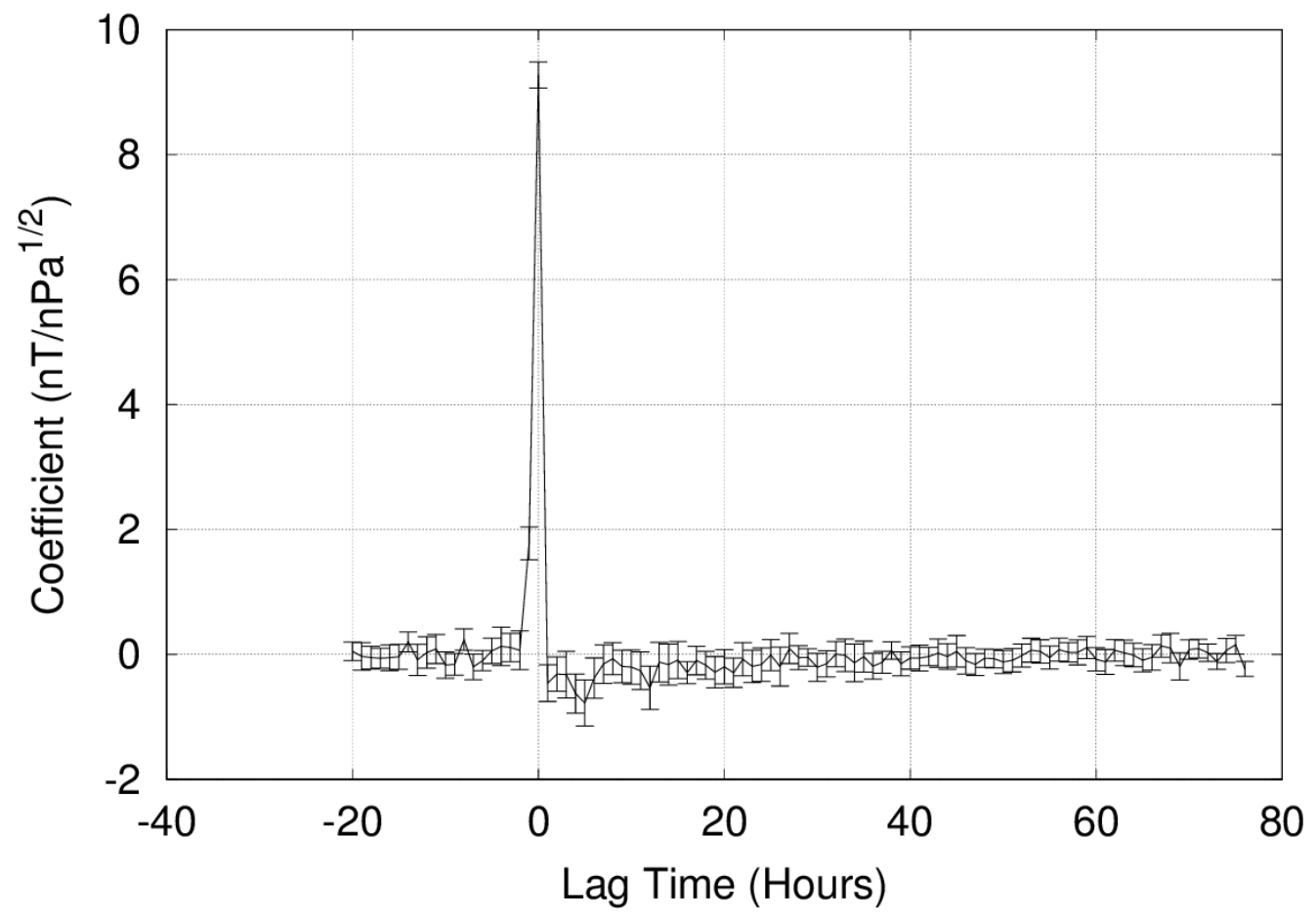

Figure 3.2: The impulse response of DST with respect to solar wind dynamic pressure. The horizontal axis represents hours of lag time. The vertical axis represents the response of DST (nT) per square rooted unit of pressure $(\mathrm{nPa})$. 


\section{IRF: DST from VBY}

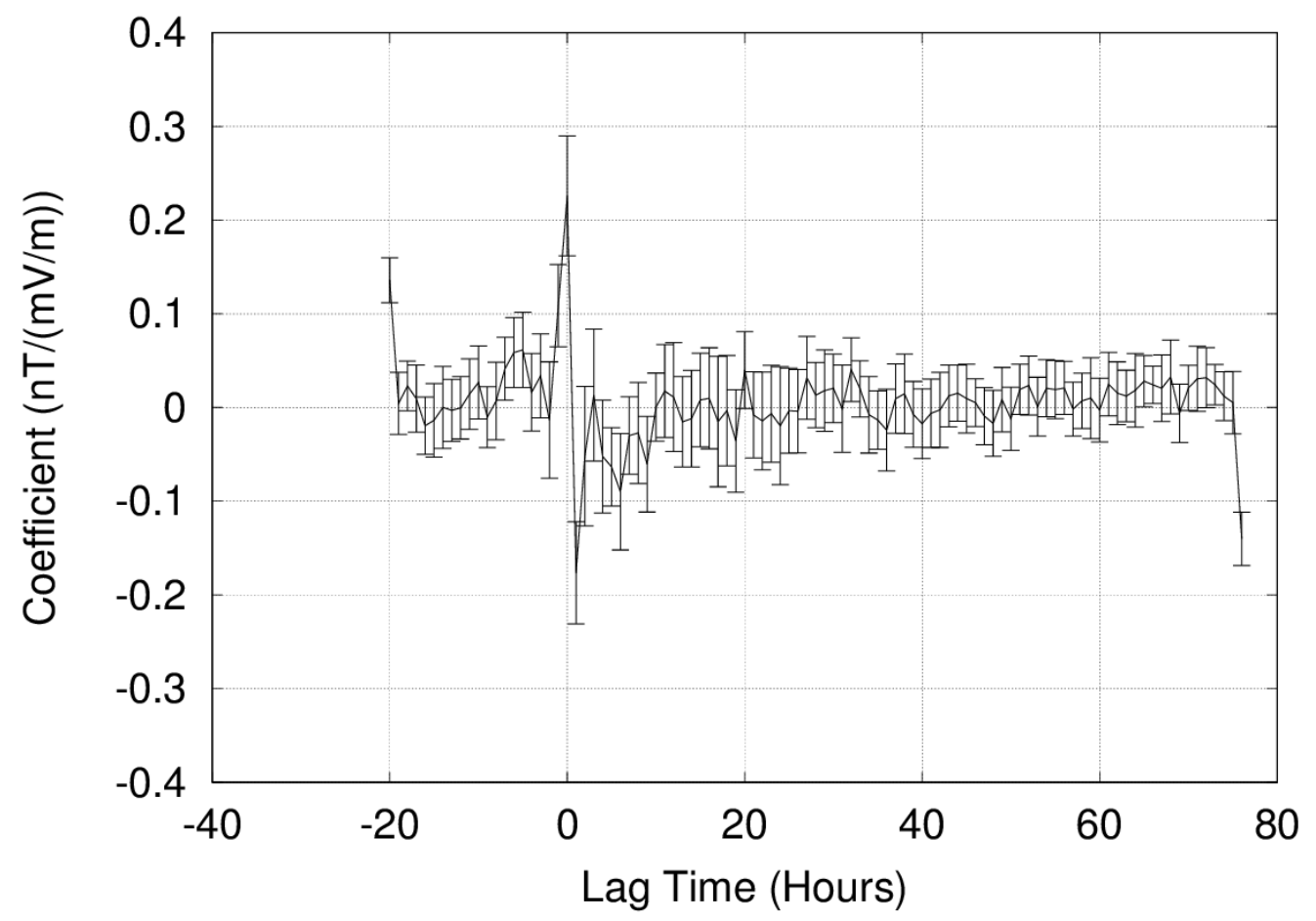

Figure 3.3: The impulse response of DST with respect to $v|B y|$. The horizontal axis represents hours of lag time. The vertical axis represents the response of DST (nT) per unit of solar wind electric field $(\mathrm{mV} / \mathrm{m})$. 
IRF: DST from VsigmaB

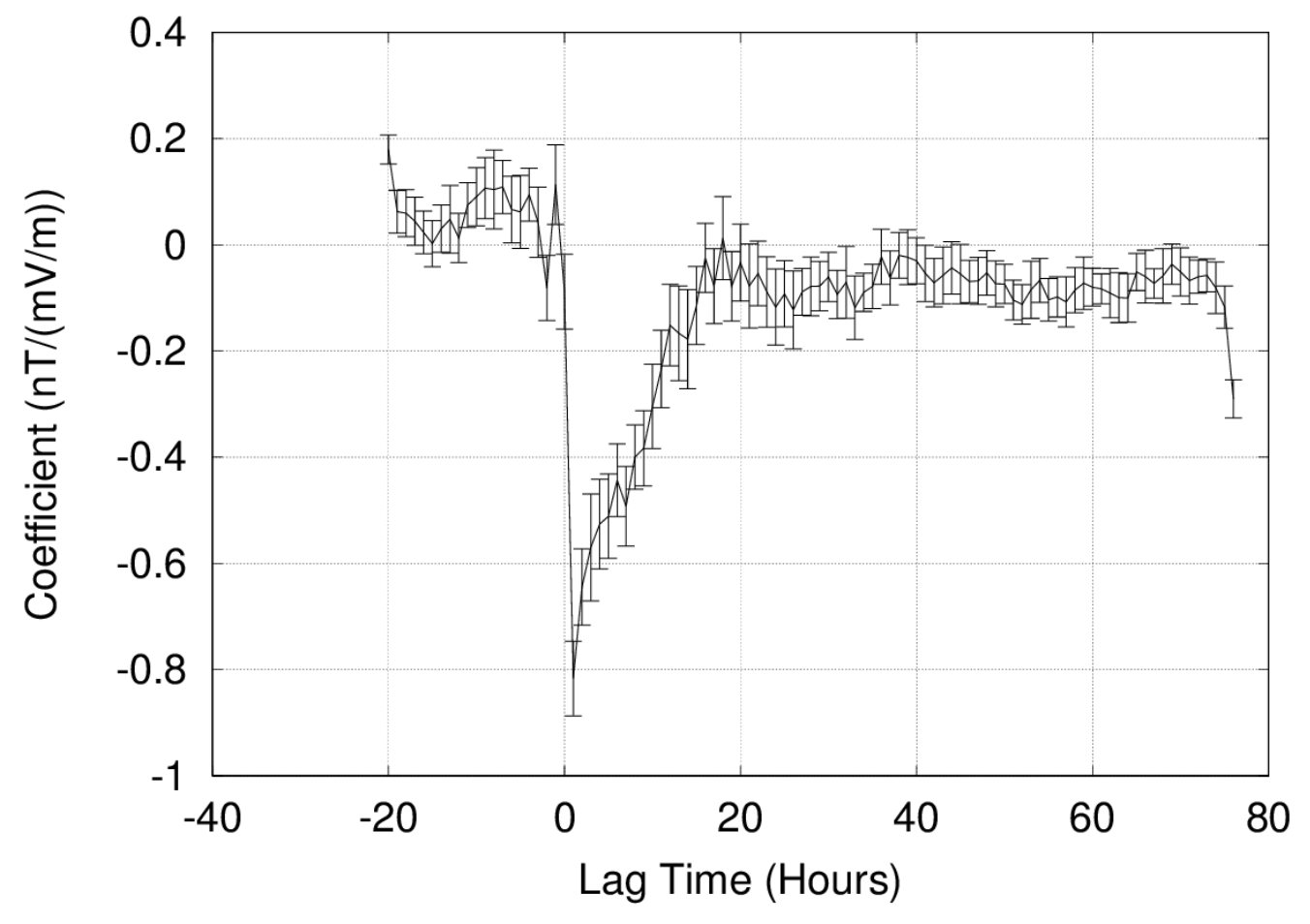

Figure 3.4: The impulse response of DST with respect to $\sigma_{B}$. The horizontal axis represents hours of lag time. The vertical axis represents the response of DST (nT) per unit of solar wind electric field variance $(\mathrm{mV} / \mathrm{m})$. 


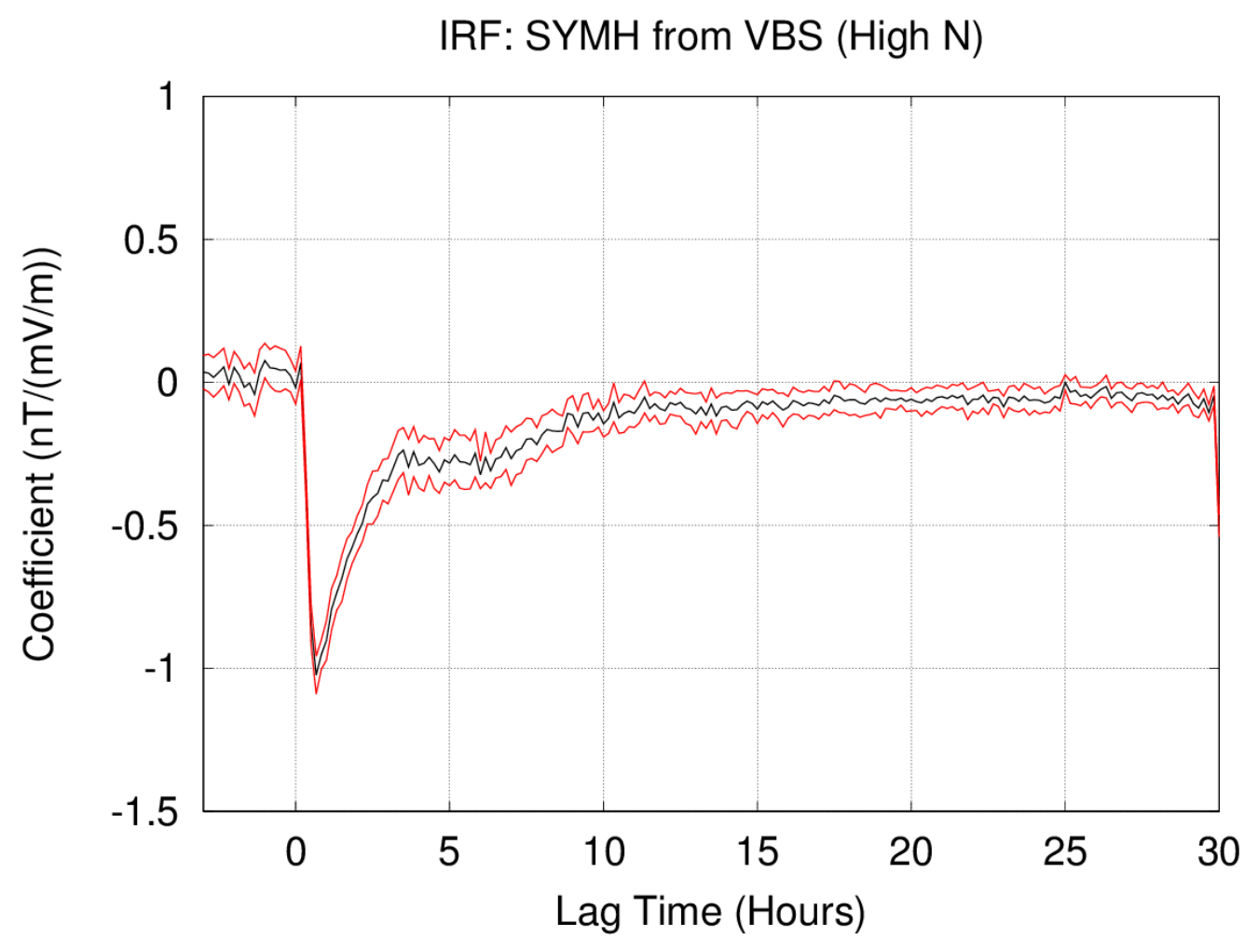

Figure 3.5: The impulse response of $\mathrm{Sym} / \mathrm{H}$ with respect to $v B_{S}$ in the high $\mathrm{SW}$ density case. 


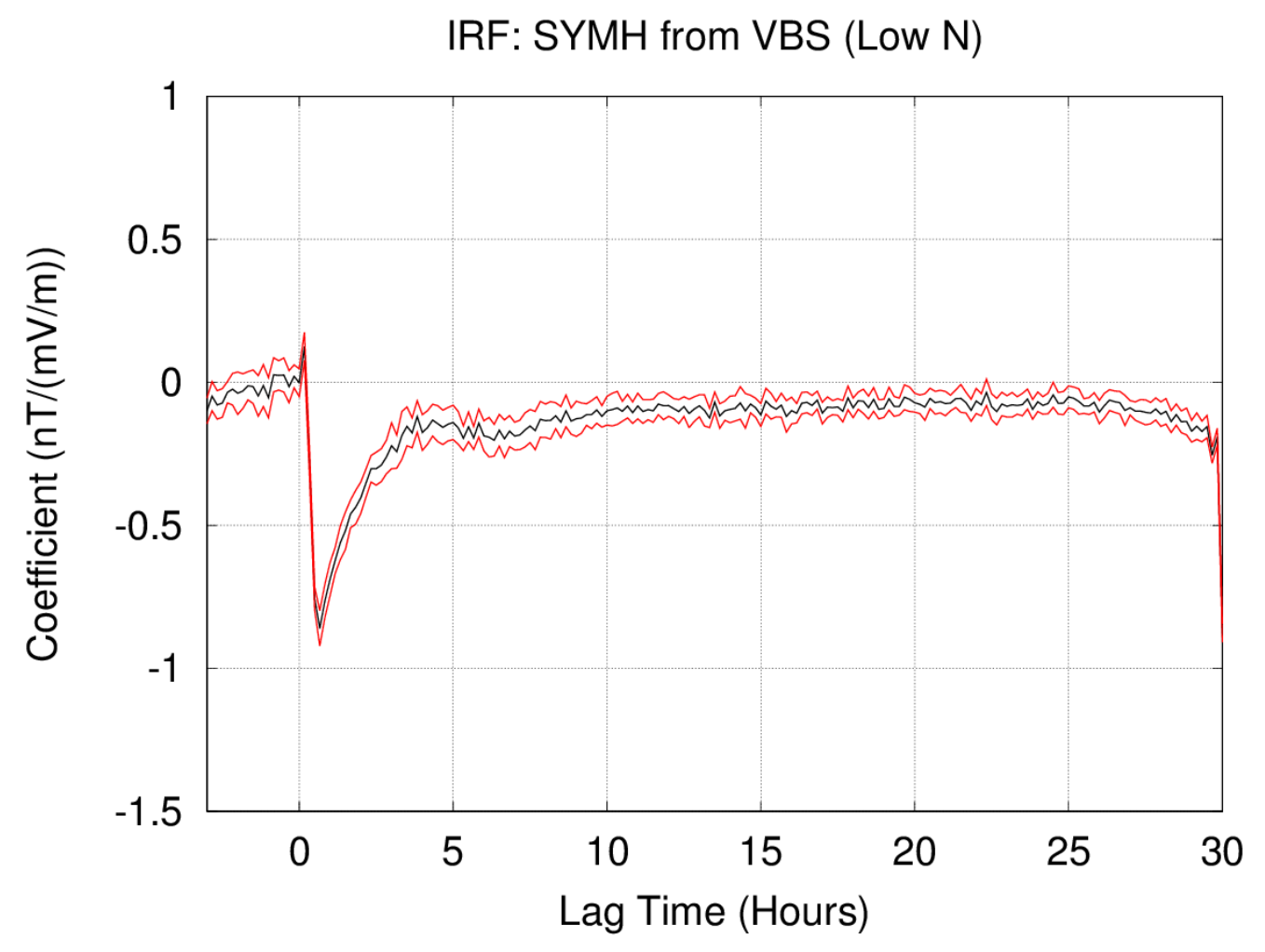

Figure 3.6: The impulse response of $\mathrm{Sym} / \mathrm{H}$ with respect to $v B_{S}$ in the low $\mathrm{SW}$ density case. 


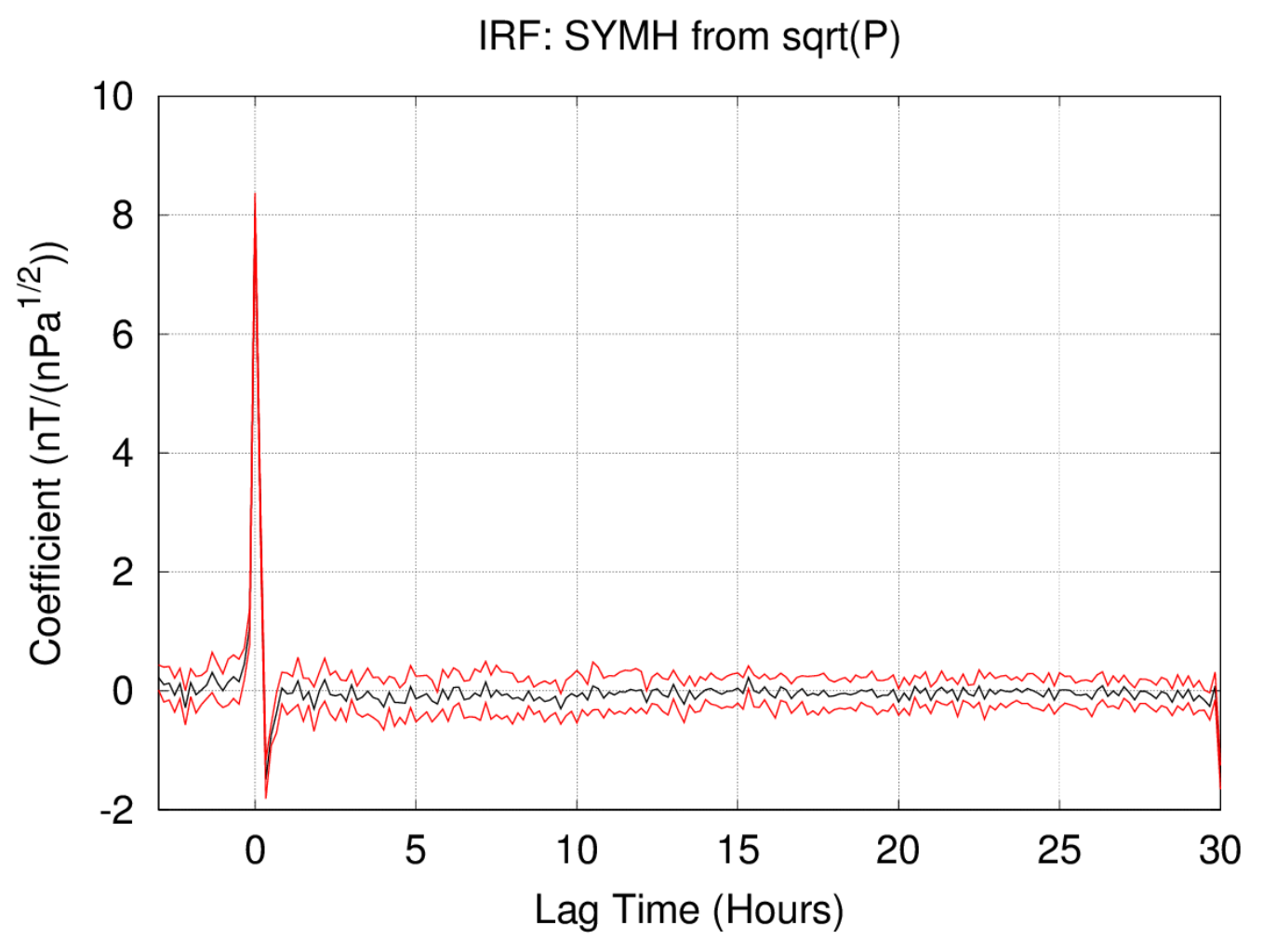

Figure 3.7: The impulse response of SYM/H with respect to $\sqrt{(} P)$. 


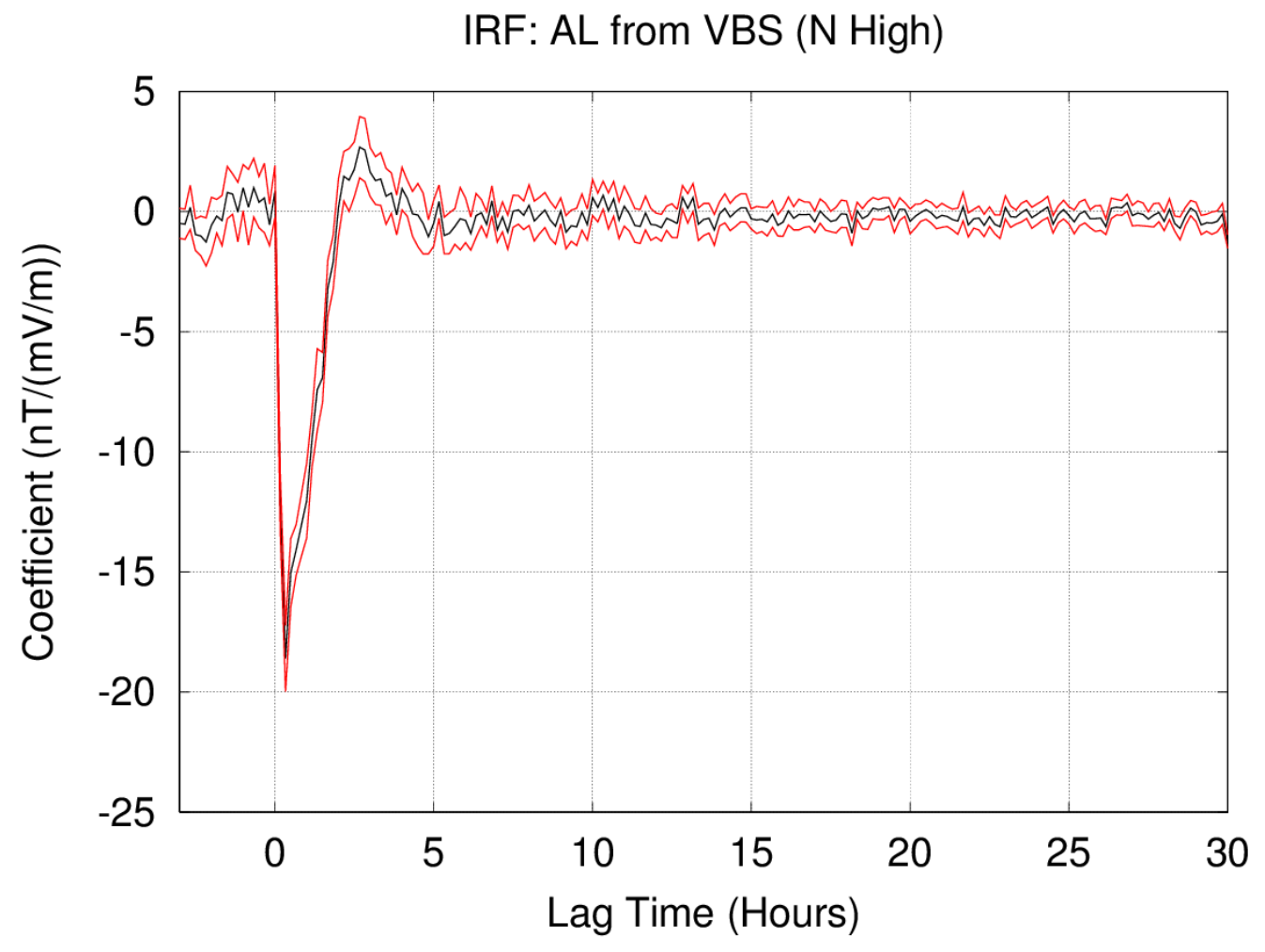

Figure 3.8: The impulse response of $\mathrm{AL}$ with respect to $v B_{S}$ in the high $\mathrm{SW}$ density case. 


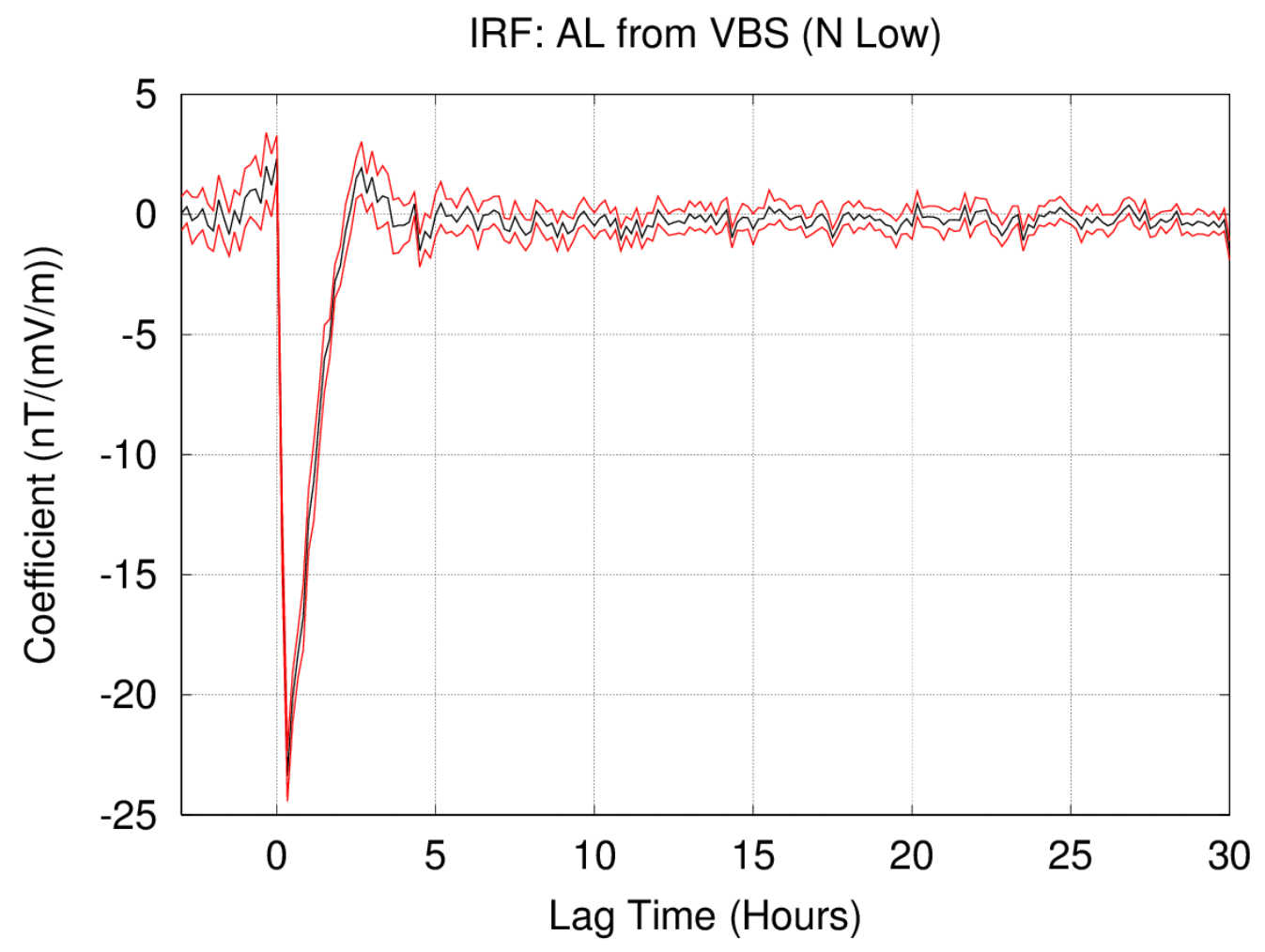

Figure 3.9: The impulse response of $\mathrm{AL}$ with respect to $v B_{S}$ in the low $\mathrm{SW}$ density case. 
IRF: $\mathrm{H}(\mathrm{ABI})$ from VBS

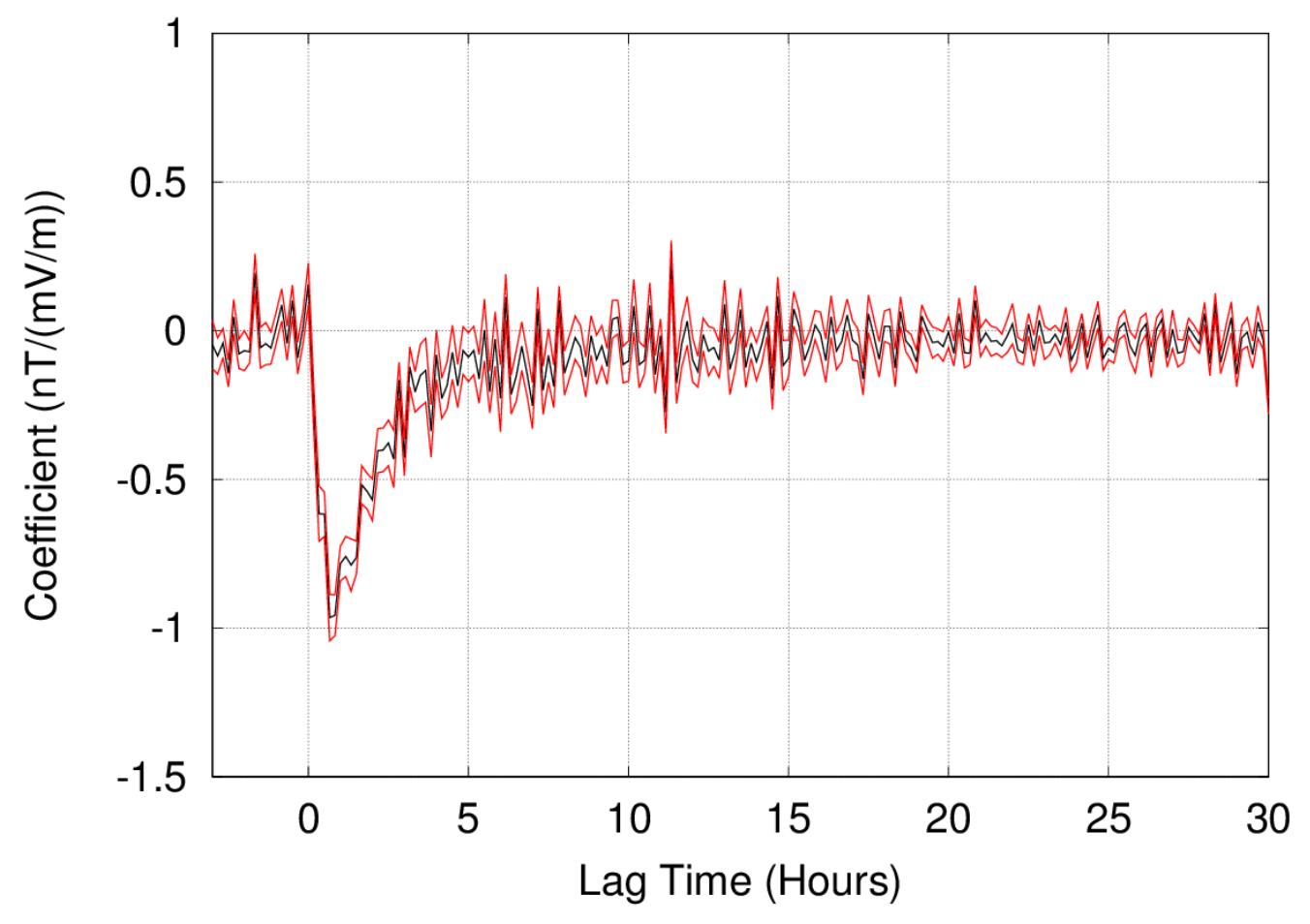

Figure 3.10: The impulse response of $\mathrm{H}(\mathrm{ABI})$, the storm-time depression predicted by the theoretical formula of Maltsev and his collaborators, with respect to $v B_{S}$. It can be seen that the ABI data set is noisy, resulting in excessive high frequency noise in this IRF plot. 


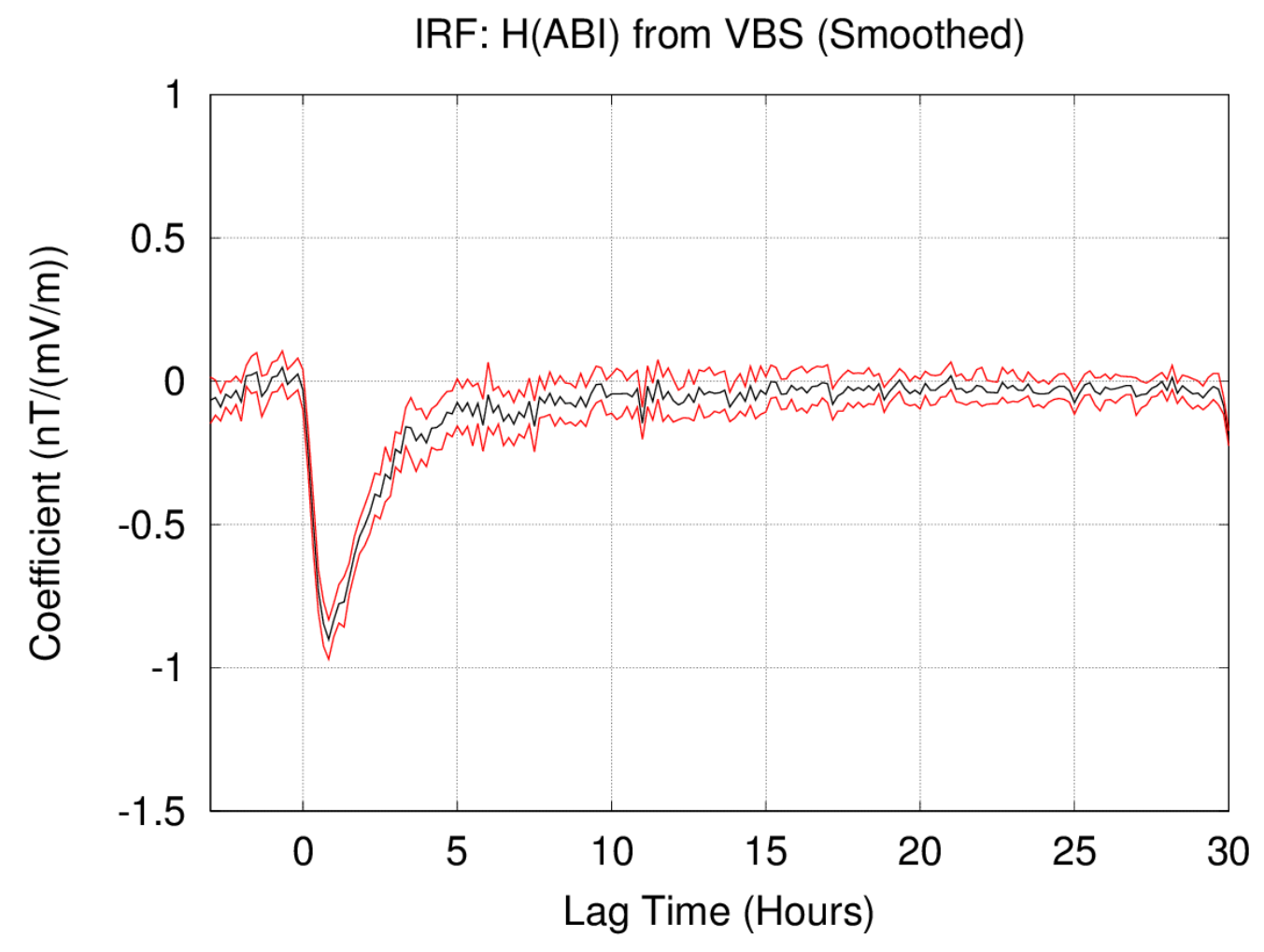

Figure 3.11: The impulse response of $\mathrm{H}(\mathrm{ABI})$ with respect to $v B_{S}$ is shown, where the coefficient are smoothed by averaging each with its nearest neighbors in lag time. 


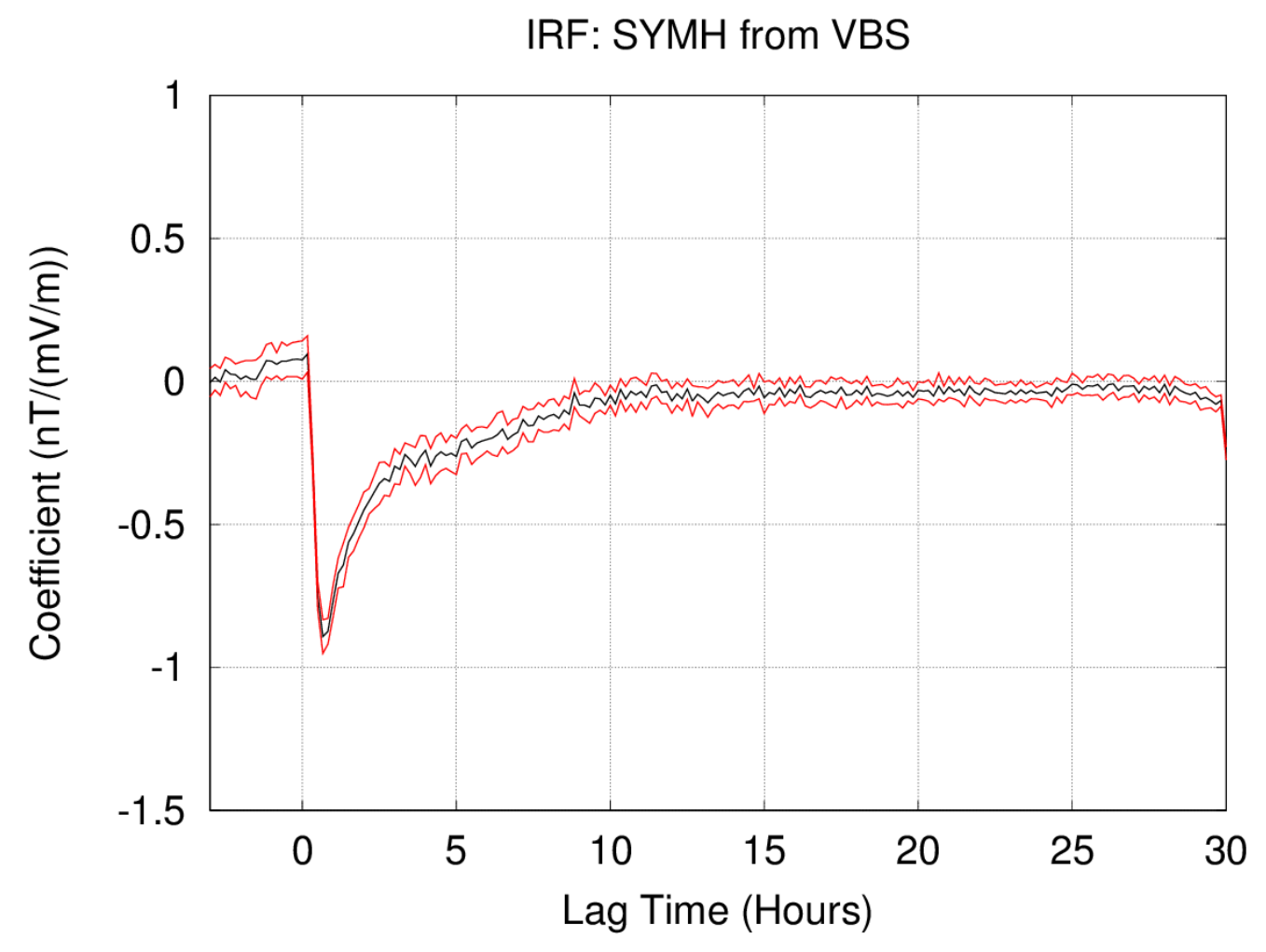

Figure 3.12: The impulse response of $\mathrm{Sym} / \mathrm{H}$ with respect to $v B_{S}$ is shown for the case where $\mathrm{AL}$ was included as an additional driver variable. Note the attenuation of the second development. 


\section{IRF: SYMH from AL}

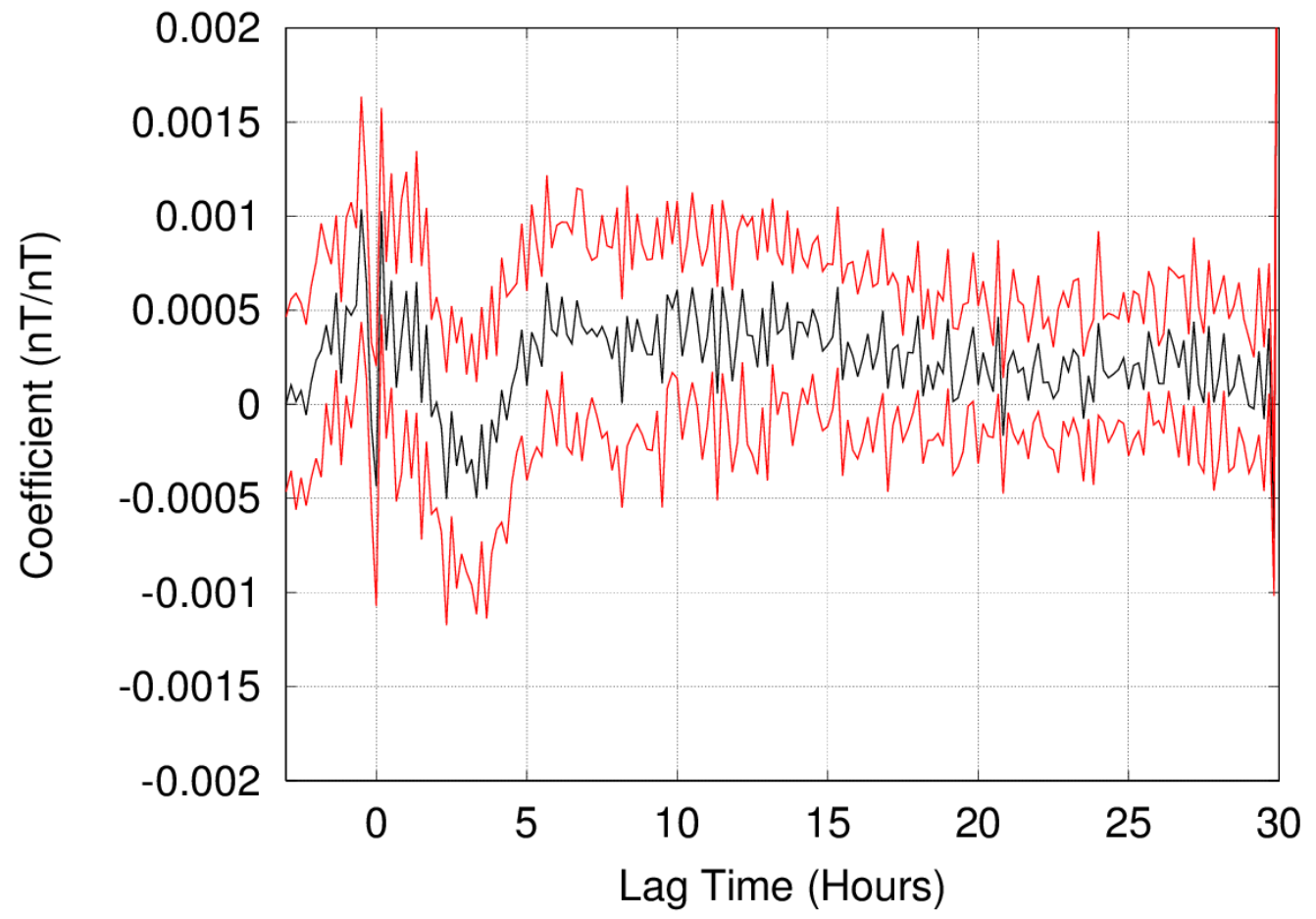

Figure 3.13: The impulse response of $\mathrm{Sym} / \mathrm{H}$ with respect to AL. Note that, although this variable is successful at removing the 2 nd development in figure 3.12 , the error bars are very large in comparison to the respective IRF coefficients. 


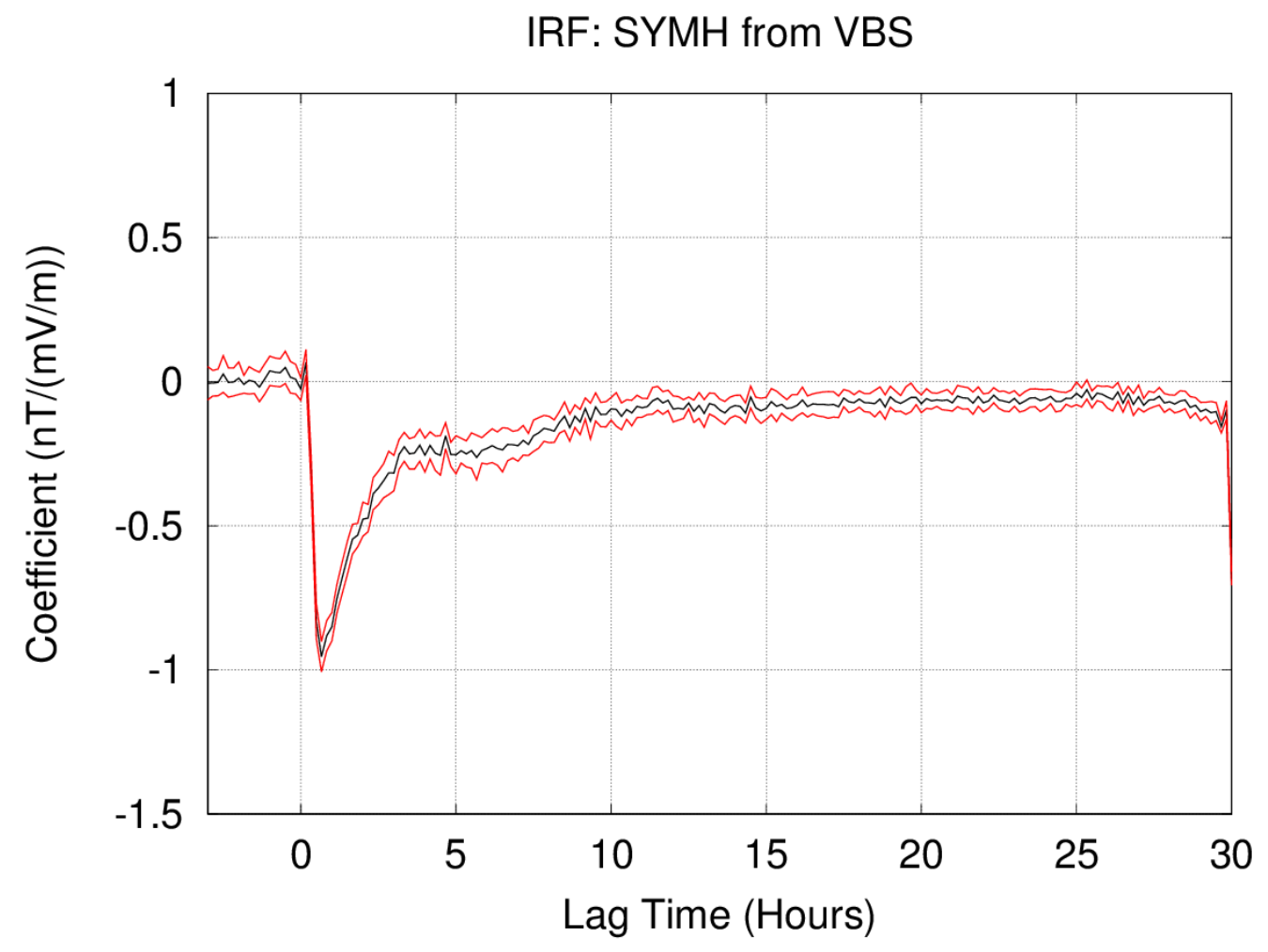

Figure 3.14: The impulse response of $\mathrm{Sym} / \mathrm{H}$ with respect to $v B_{S}$ is shown for the case where $\mathrm{AL}$ was not included as an additional driver variable for comparison with figure 3.12. Note the 2nd development at the lag time of 5-6 hours. 


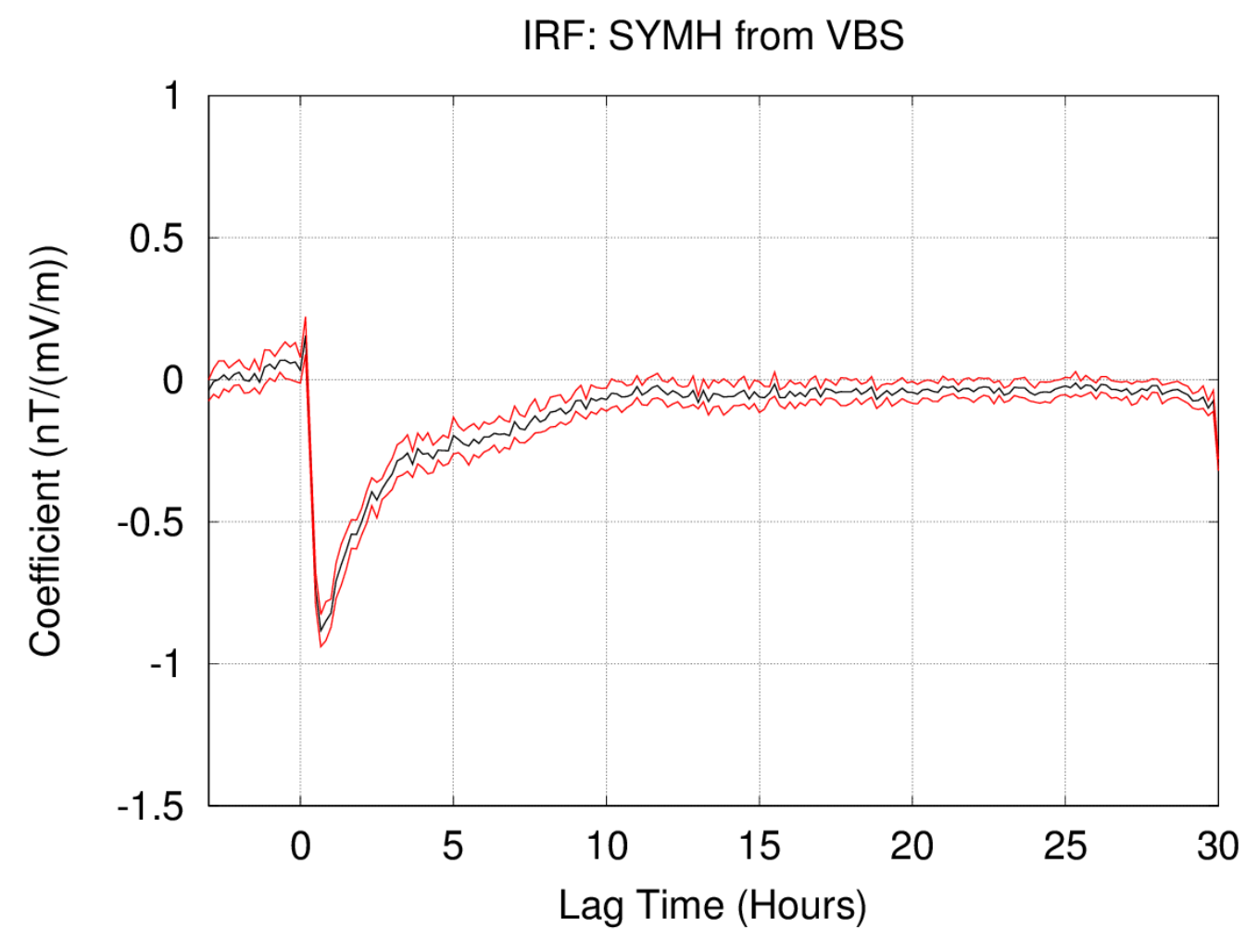

Figure 3.15: The impulse response of $\mathrm{Sym} / \mathrm{H}$ with respect to $v B_{S}$ is shown for the case where $\delta A L$ was included as an additional driver variable. Note the attenuation of the second development. 


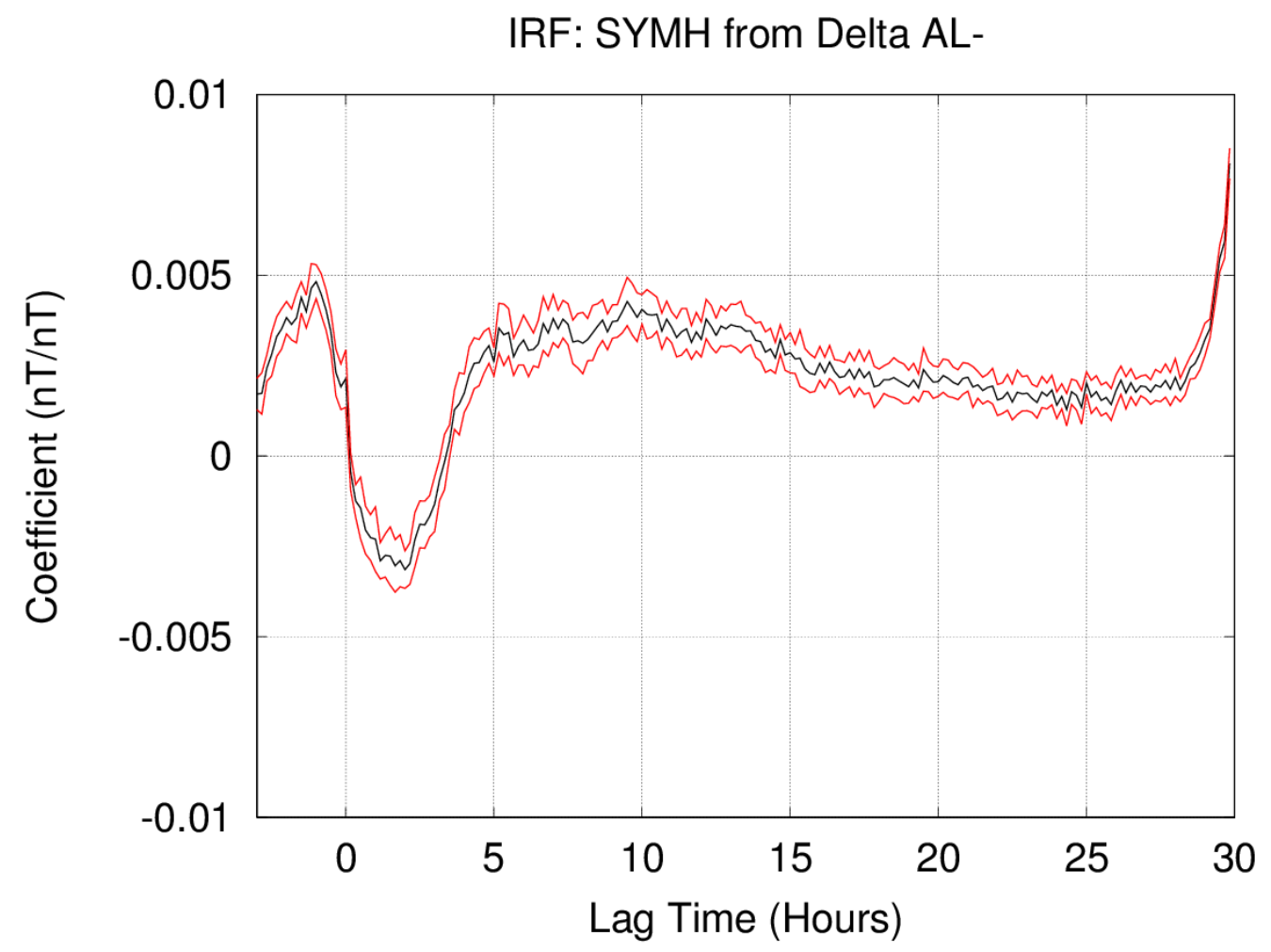

Figure 3.16: The impulse response of $\mathrm{Sym} / \mathrm{H}$ with respect to negative $\delta A L$ is shown. A positive (negative) value means the unloading produces a strengthening (weakening) of the index at that lag time. 


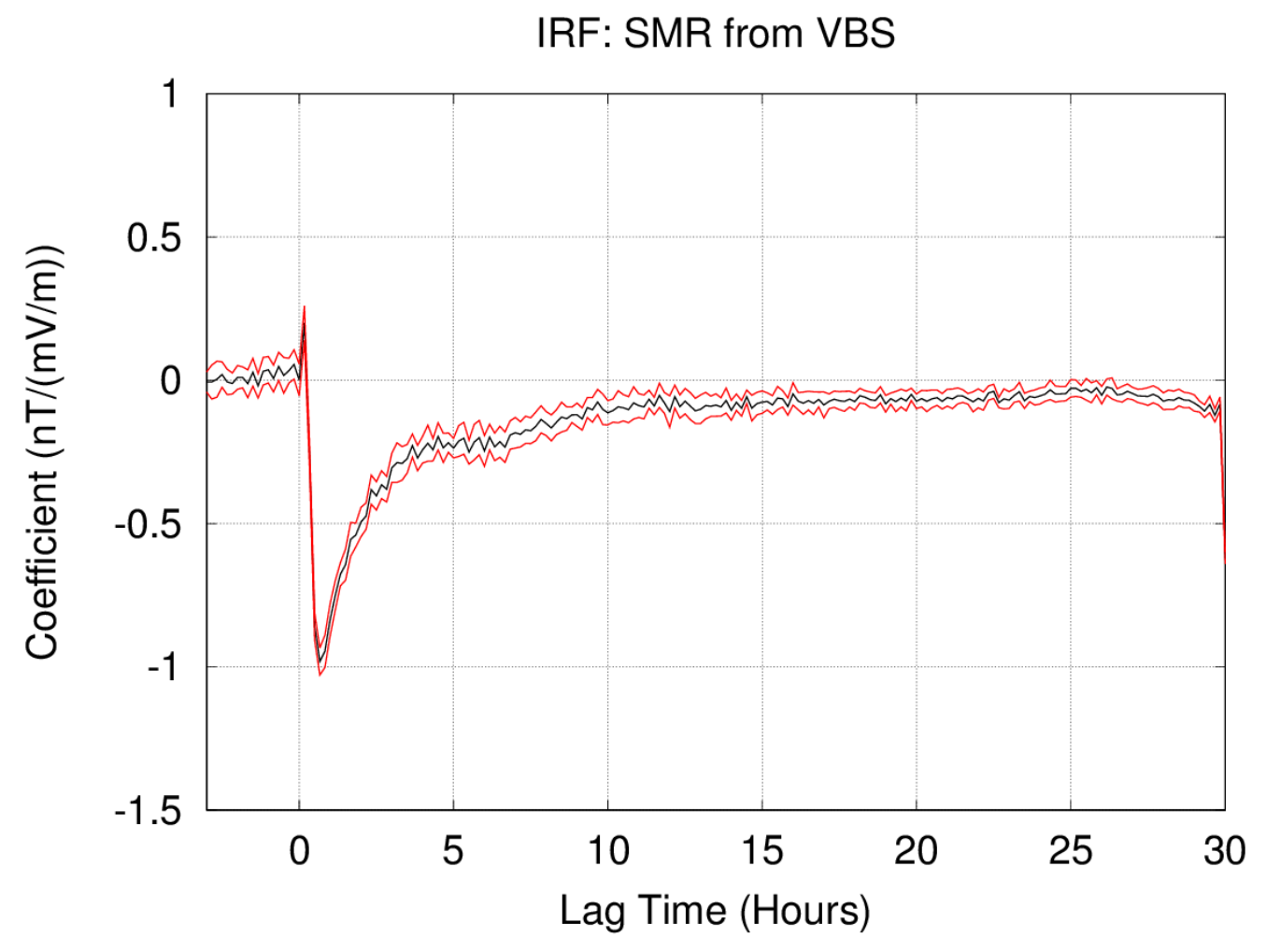

Figure 3.17: The impulse response of SMR with respect to $\mathrm{v} B_{S}$. The IRF for this index is similar to that of Sym/H. 


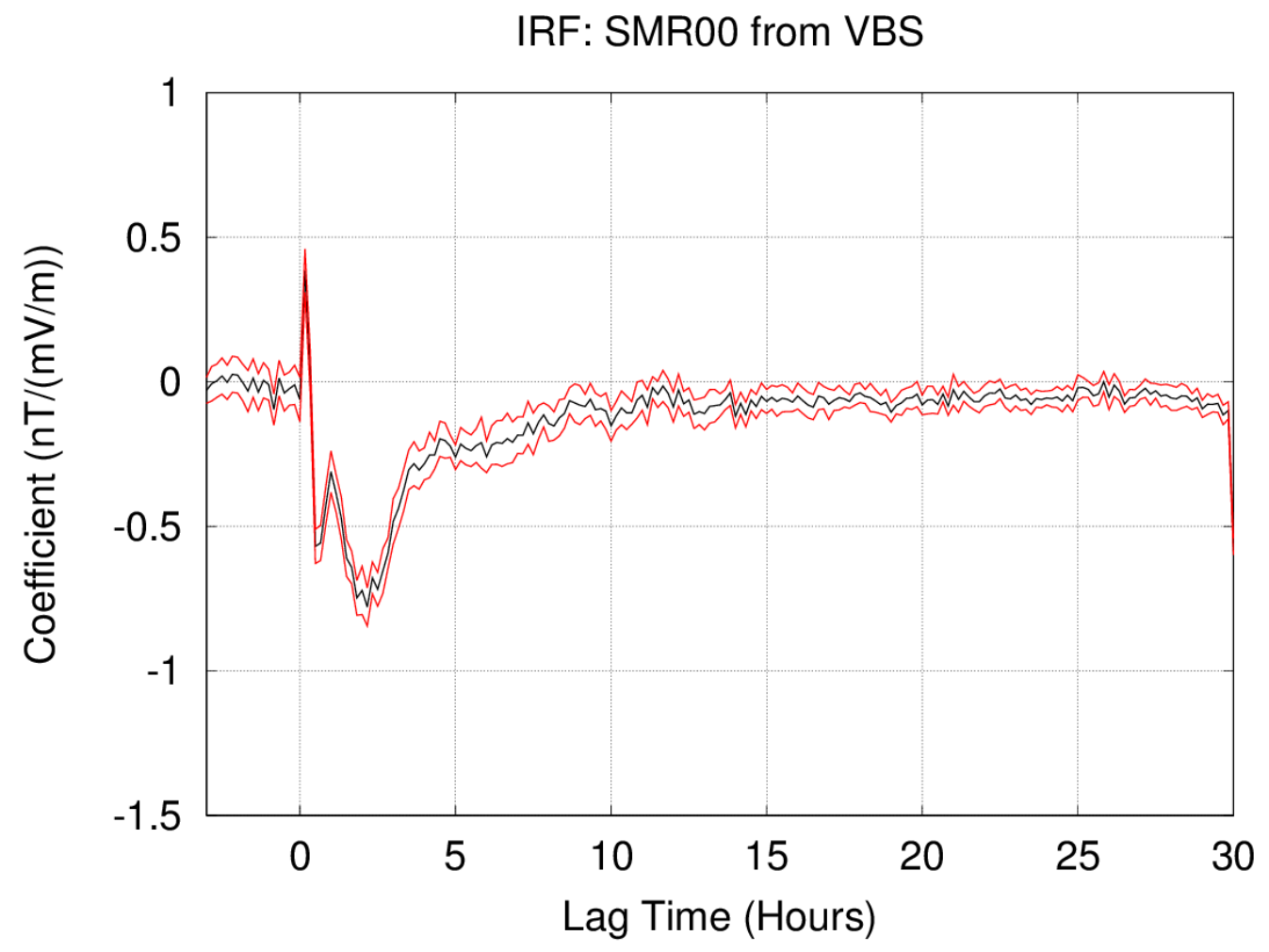

Figure 3.18: The impulse response of midnight sector SMR00 with respect to $\mathrm{v} B_{S}$. 


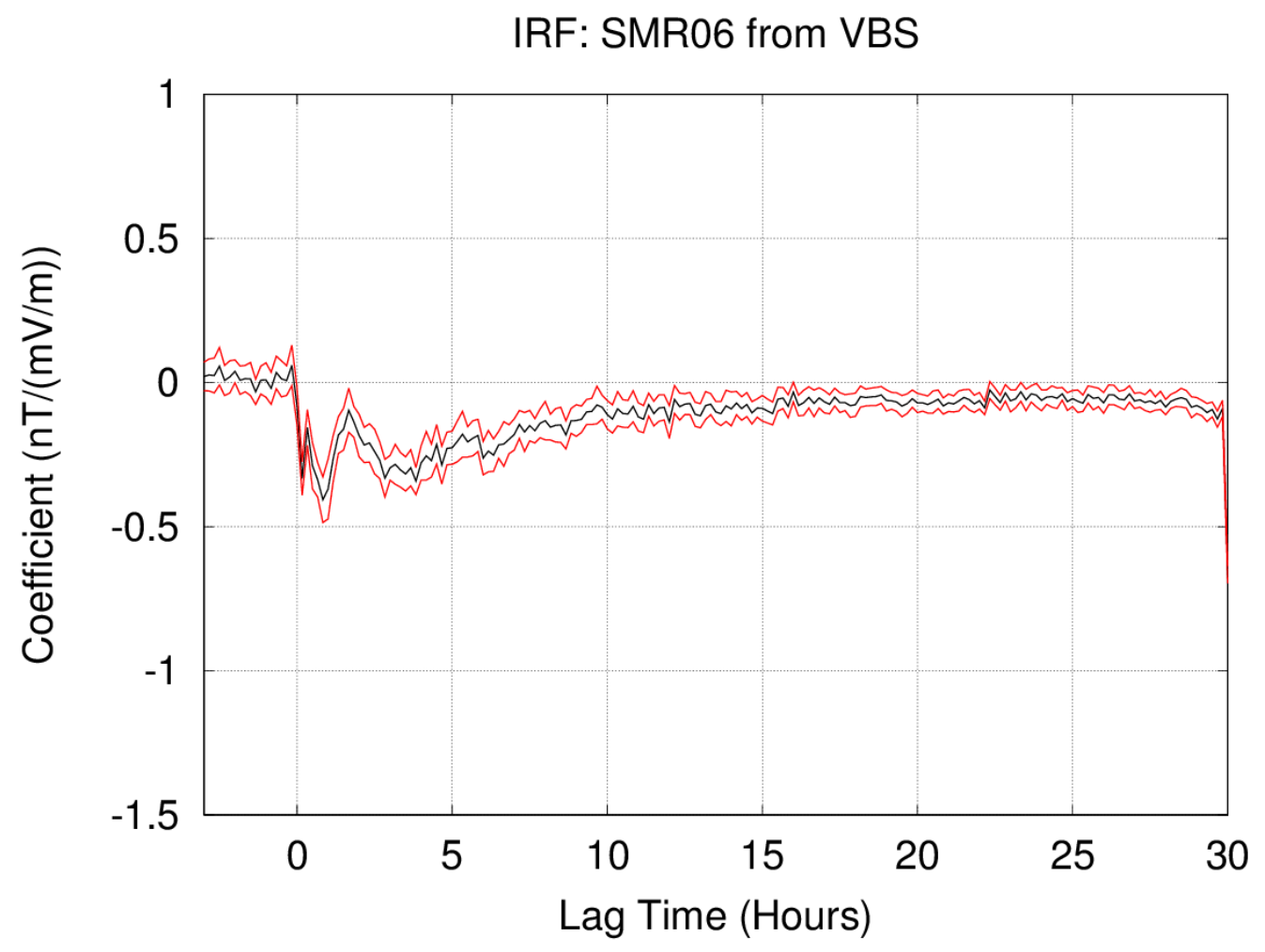

Figure 3.19: The impulse response of dawn sector SMR06 with respect to $\mathrm{v} B_{S}$. 


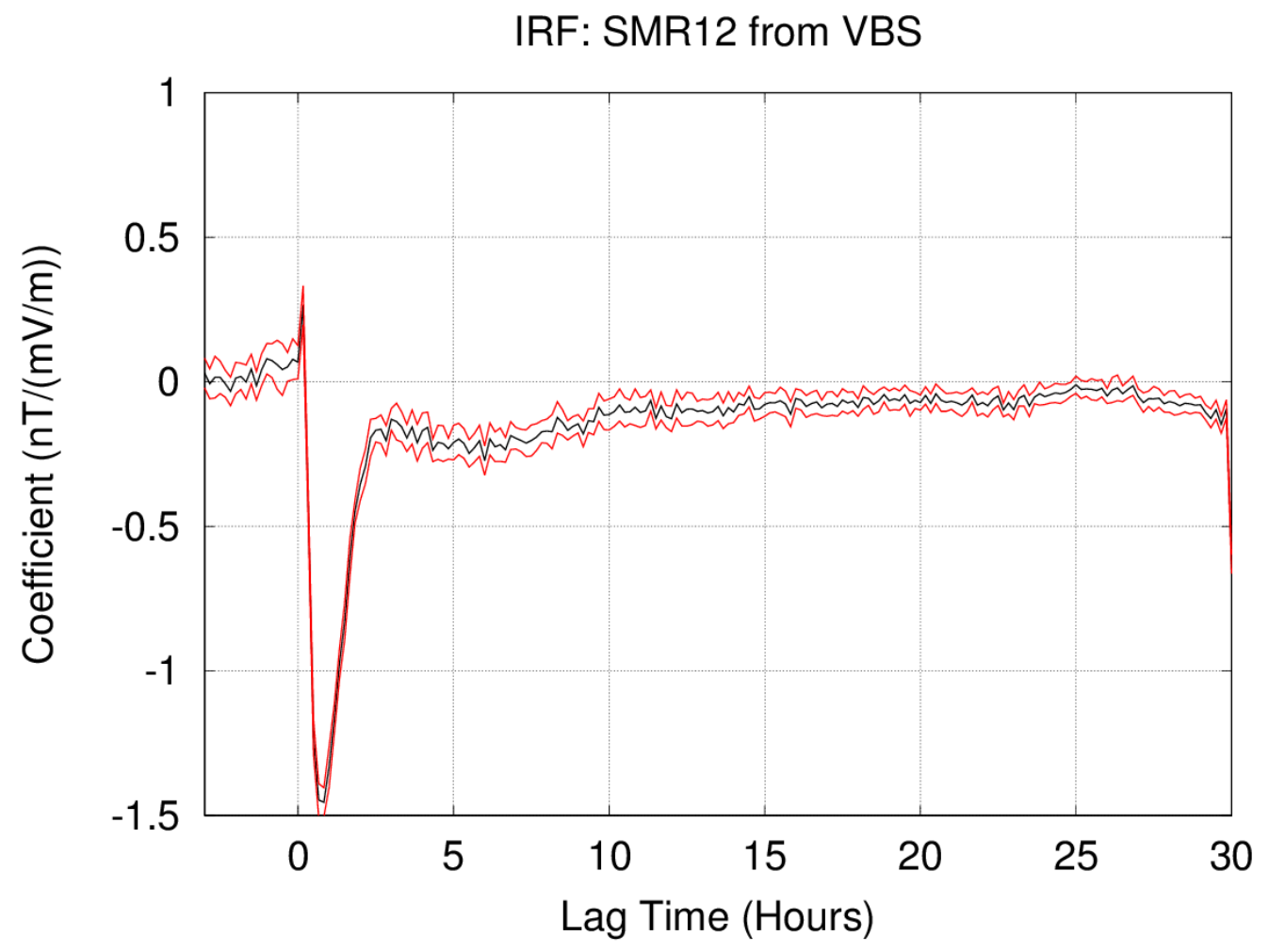

Figure 3.20: The impulse response of noon sector SMR12 with respect to $\mathrm{v} B_{S}$. 


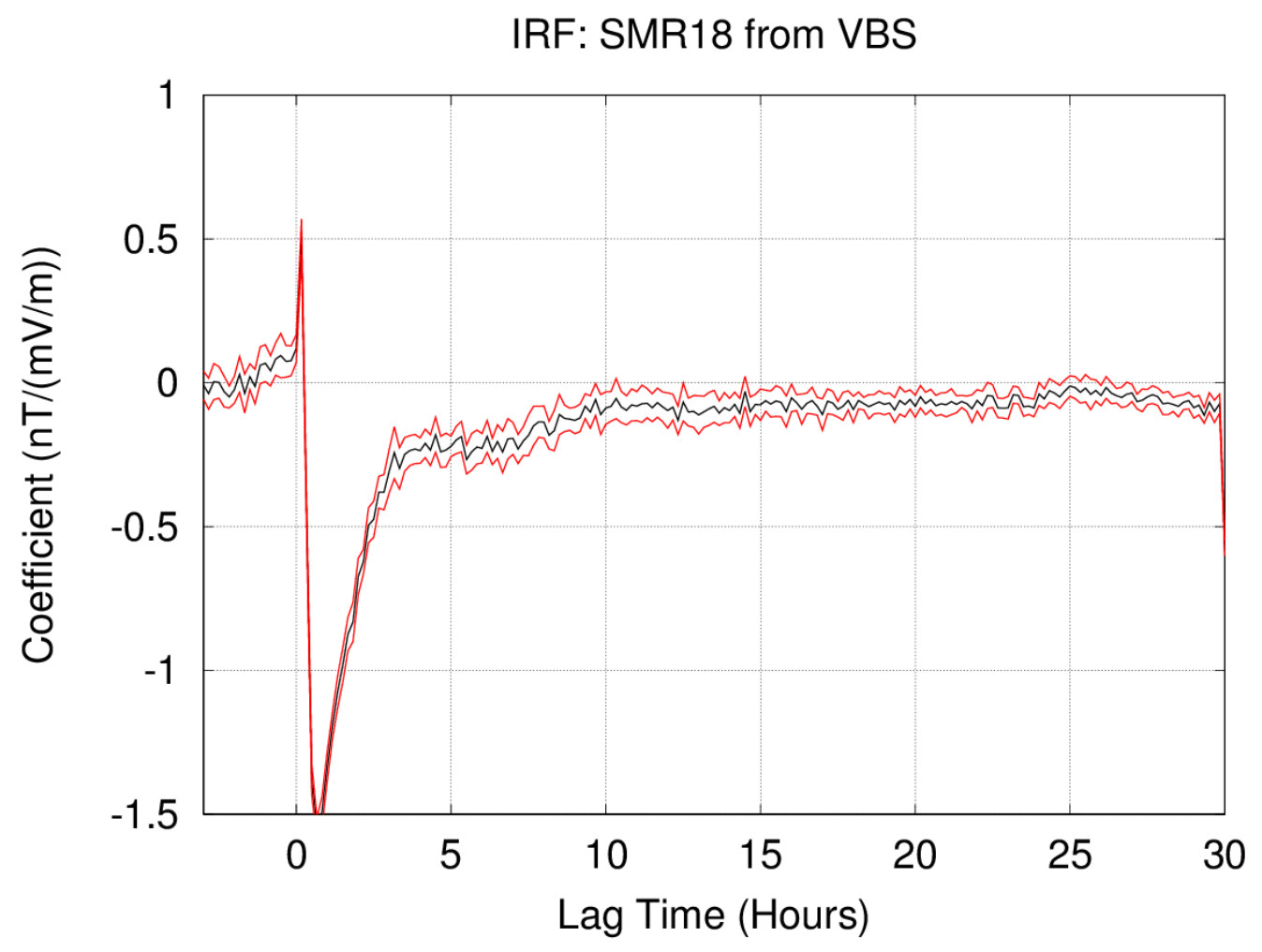

Figure 3.21: The impulse response of dusk sector SMR18 with respect to $\mathrm{v} B_{S}$. 
IRF: SMR00 from sqrt(P)

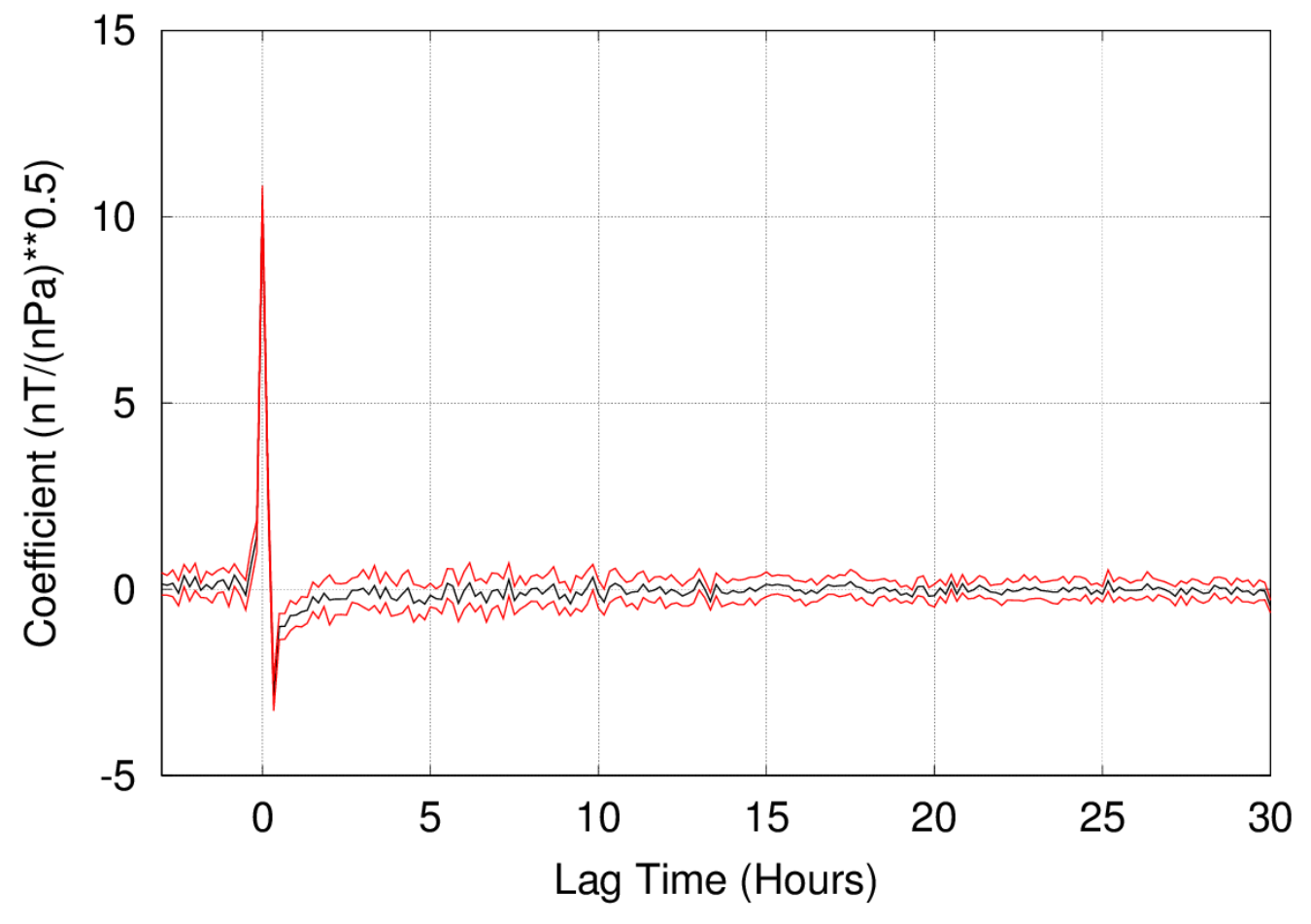

Figure 3.22: The impulse response of midnight sector SMR00 with respect to $\sqrt{P}$. 
IRF: SMR06 from sqrt(P)

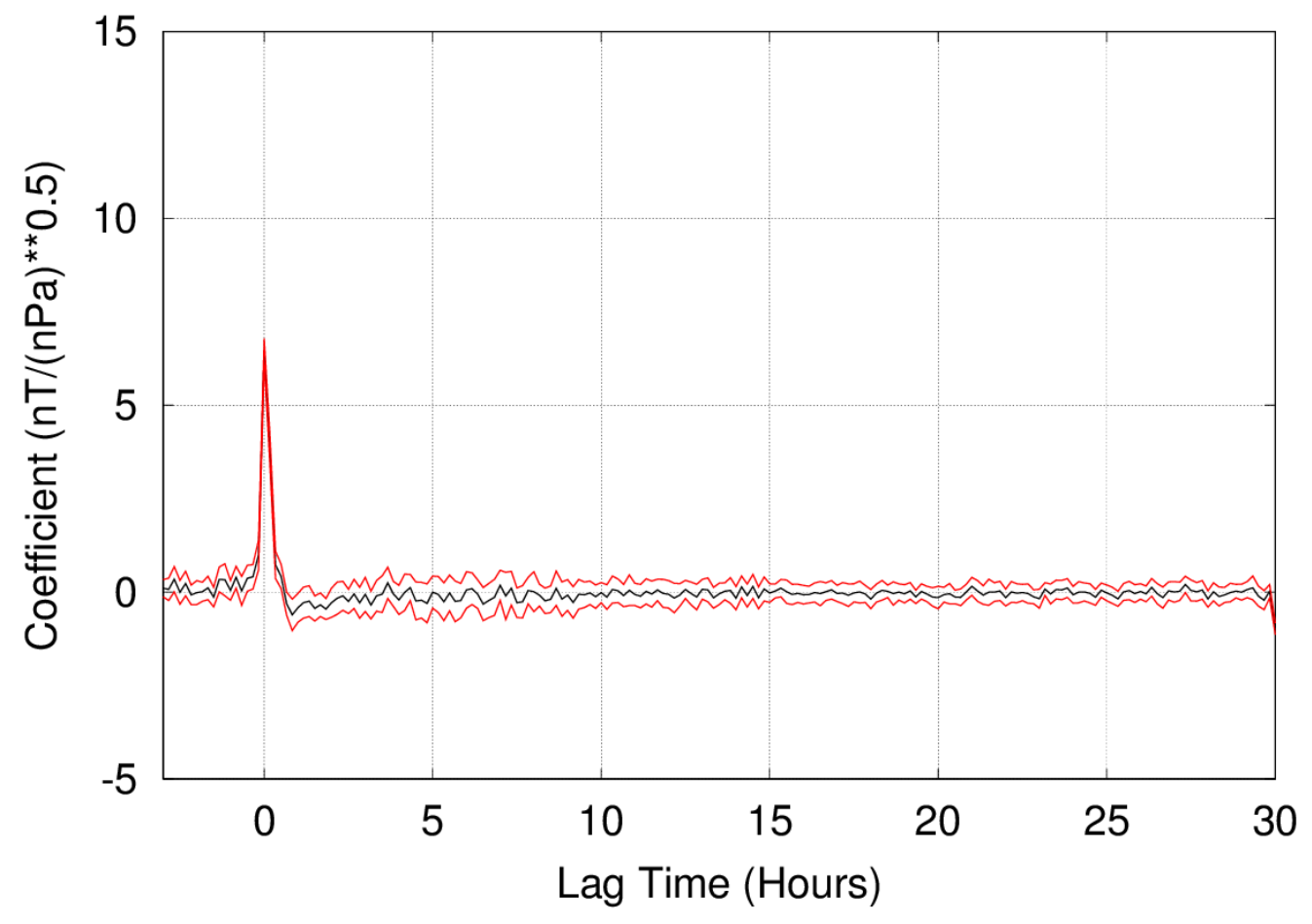

Figure 3.23: The impulse response of dawn sector SMR06 with respect to $\sqrt{P}$. 
IRF: SMR12 from sqrt(P)

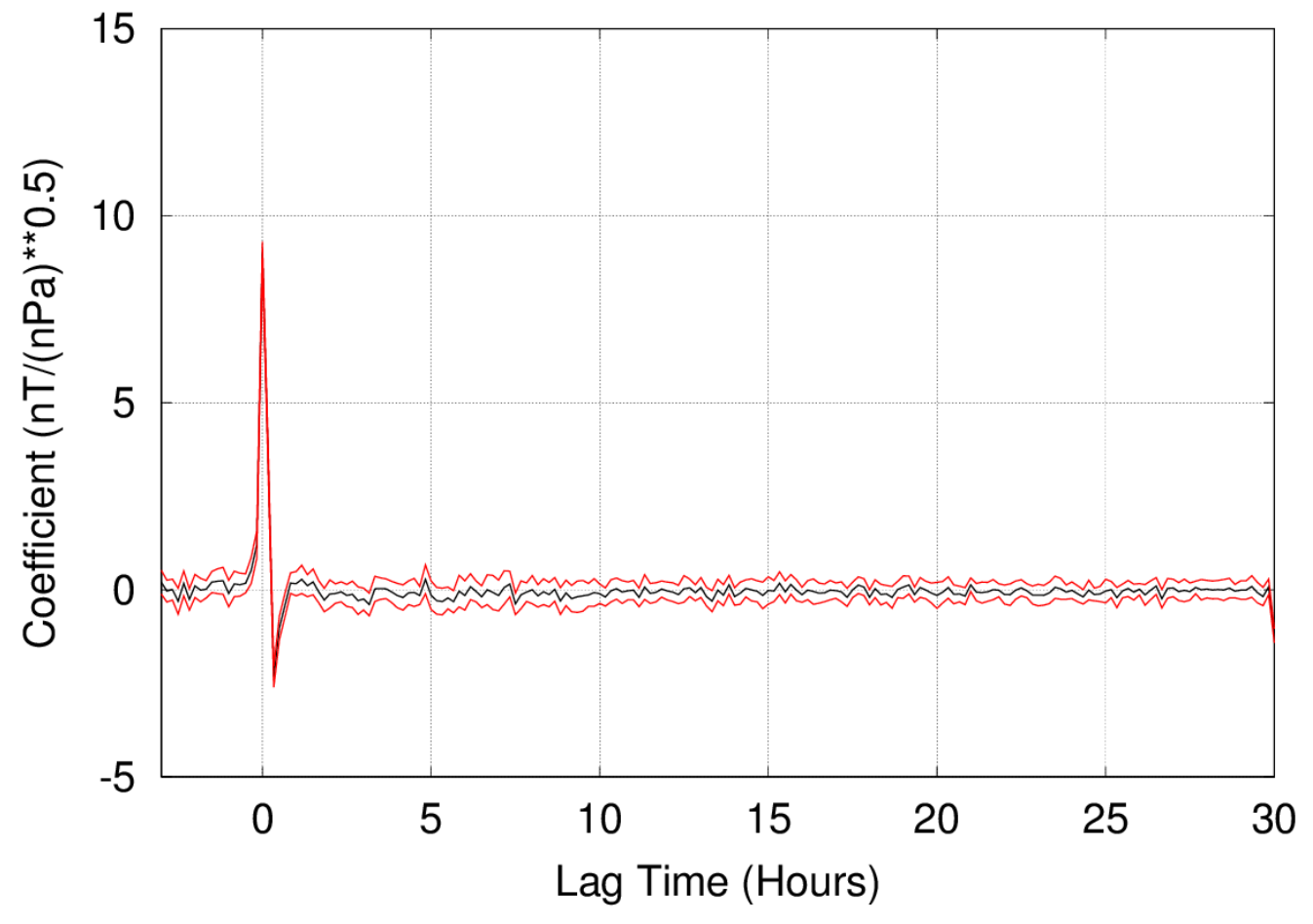

Figure 3.24: The impulse response of noon sector SMR12 with respect to $\sqrt{P}$. 
IRF: SMR18 from sqrt(P)

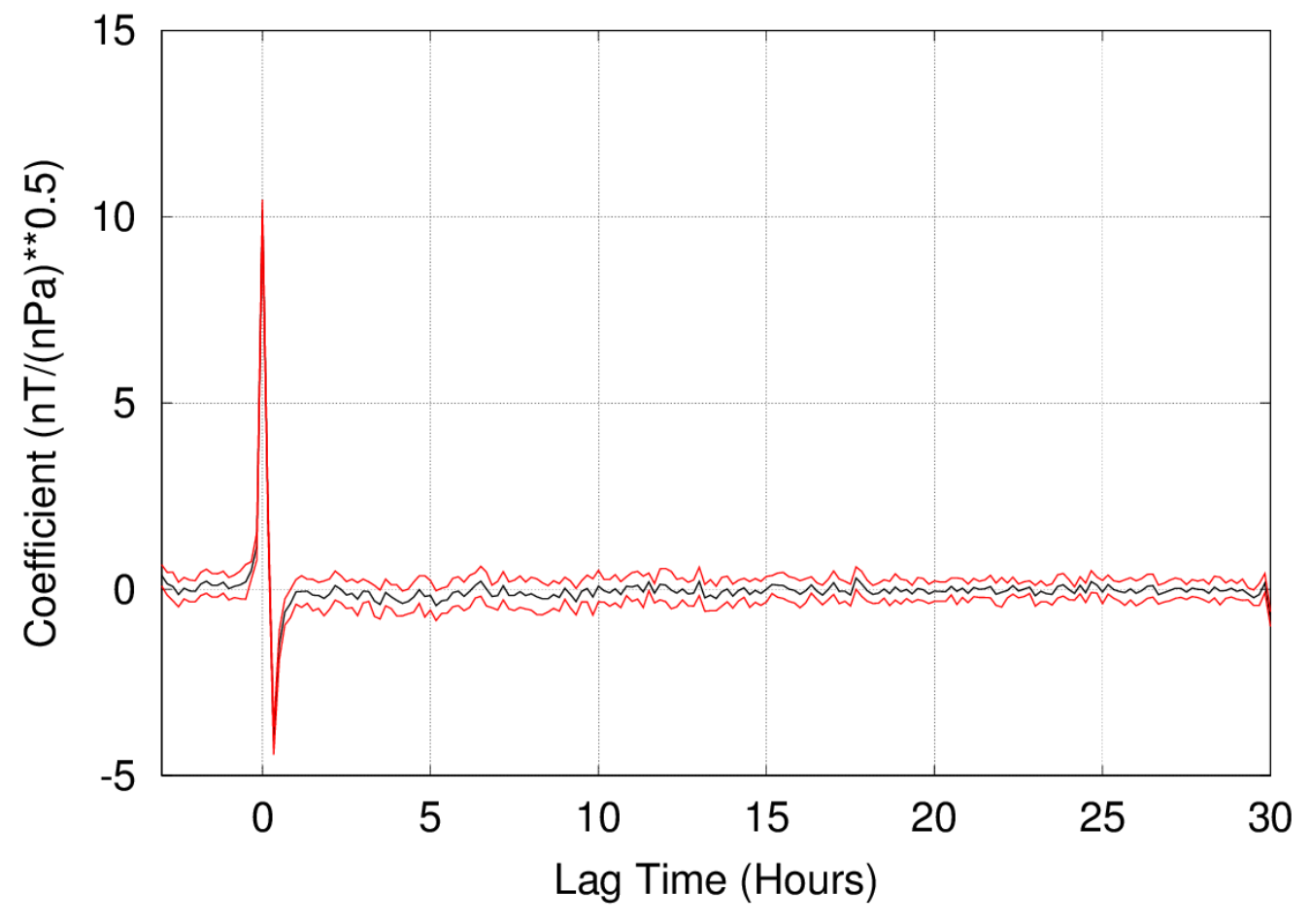

Figure 3.25: The impulse response of dusk sector SMR18 with respect to $\sqrt{P}$. 


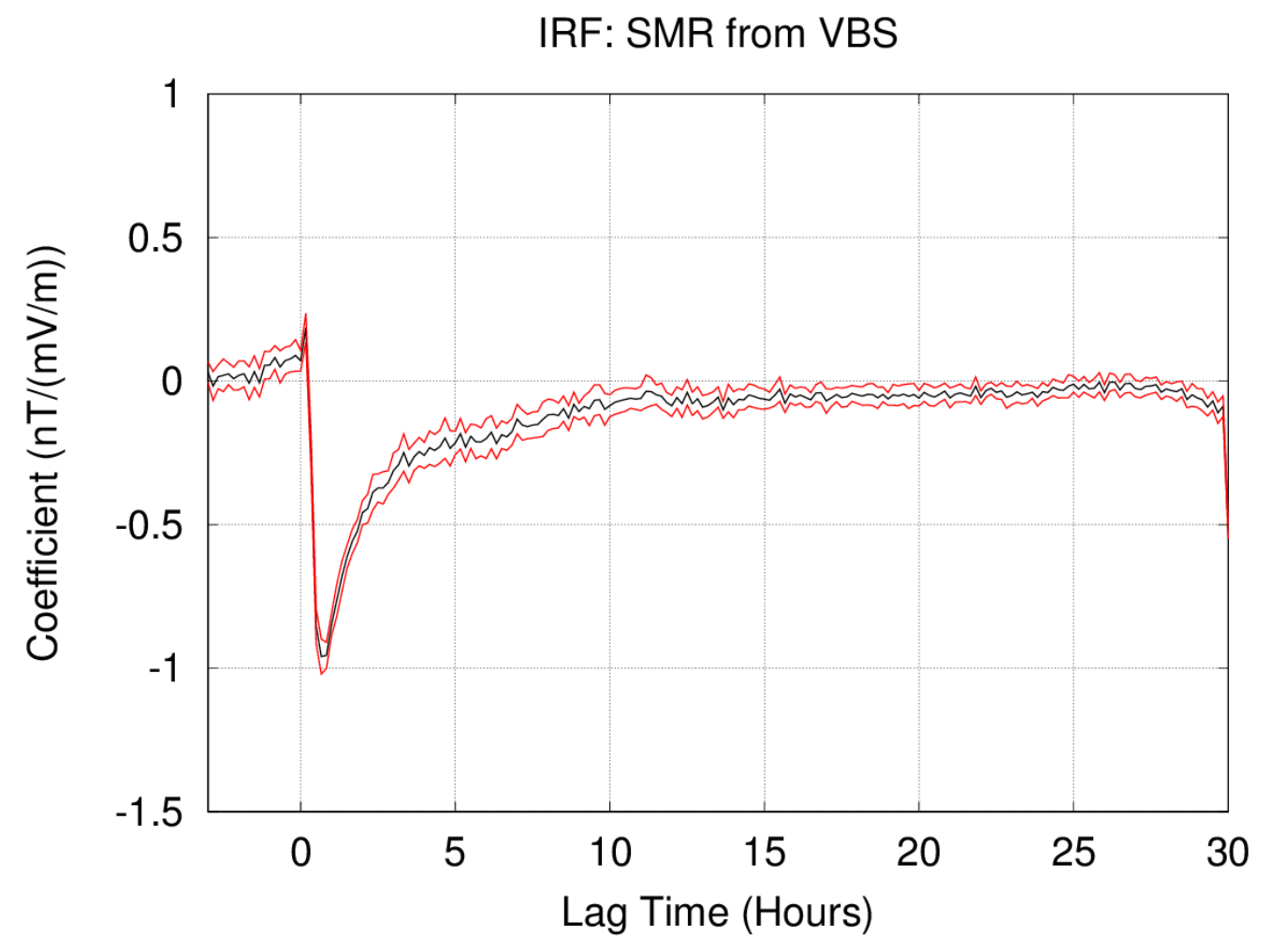

Figure 3.26: The impulse response of SMR with respect to $\mathrm{v} B_{S}$, where the negative changes in $\mathrm{AL}$ are added as an additional regression variable. 


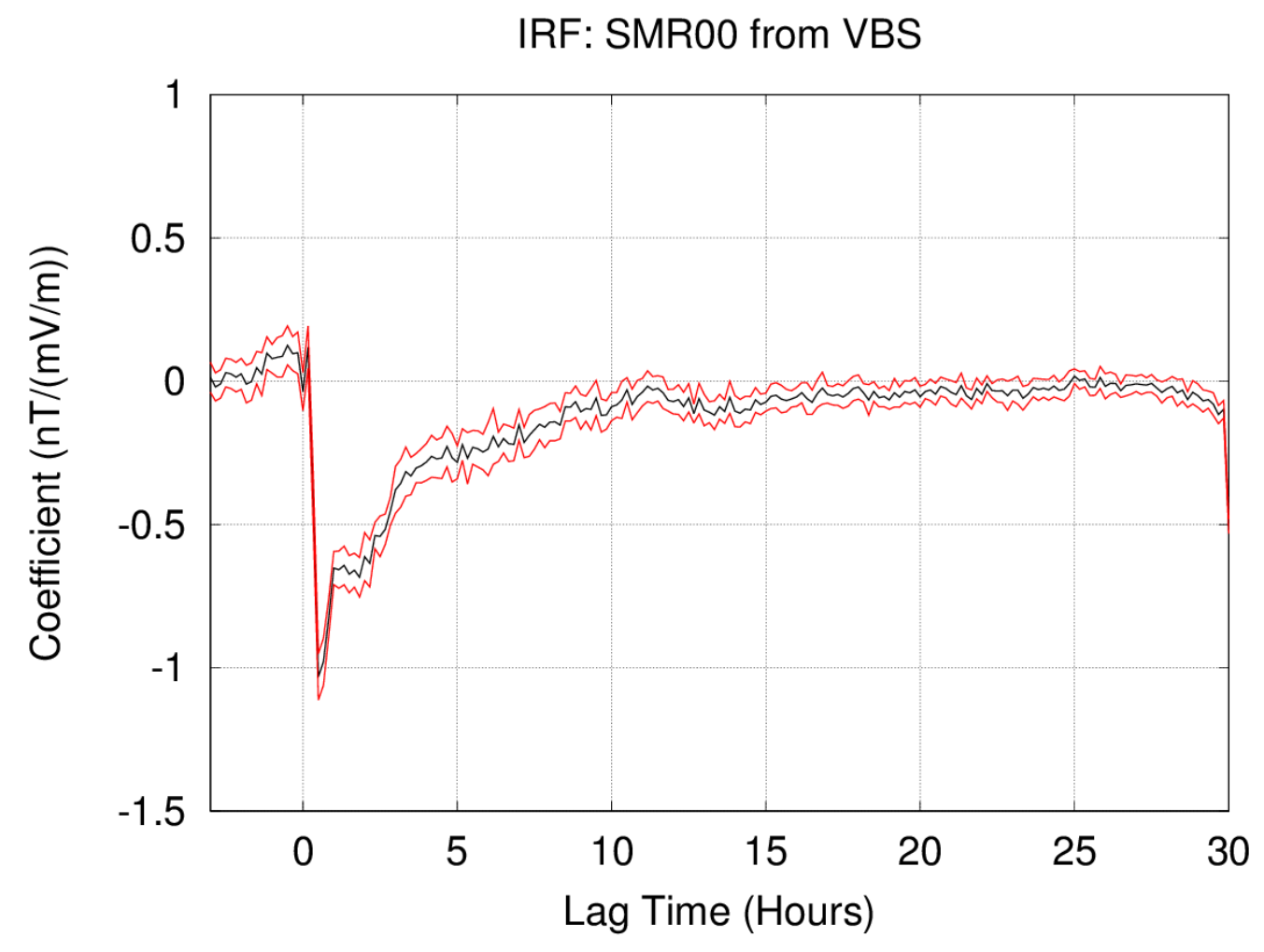

Figure 3.27: The impulse response of midnight sector SMR00 with respect to $\mathrm{v} B_{S}$, where the negative changes in AL are added as an additional regression variable. 


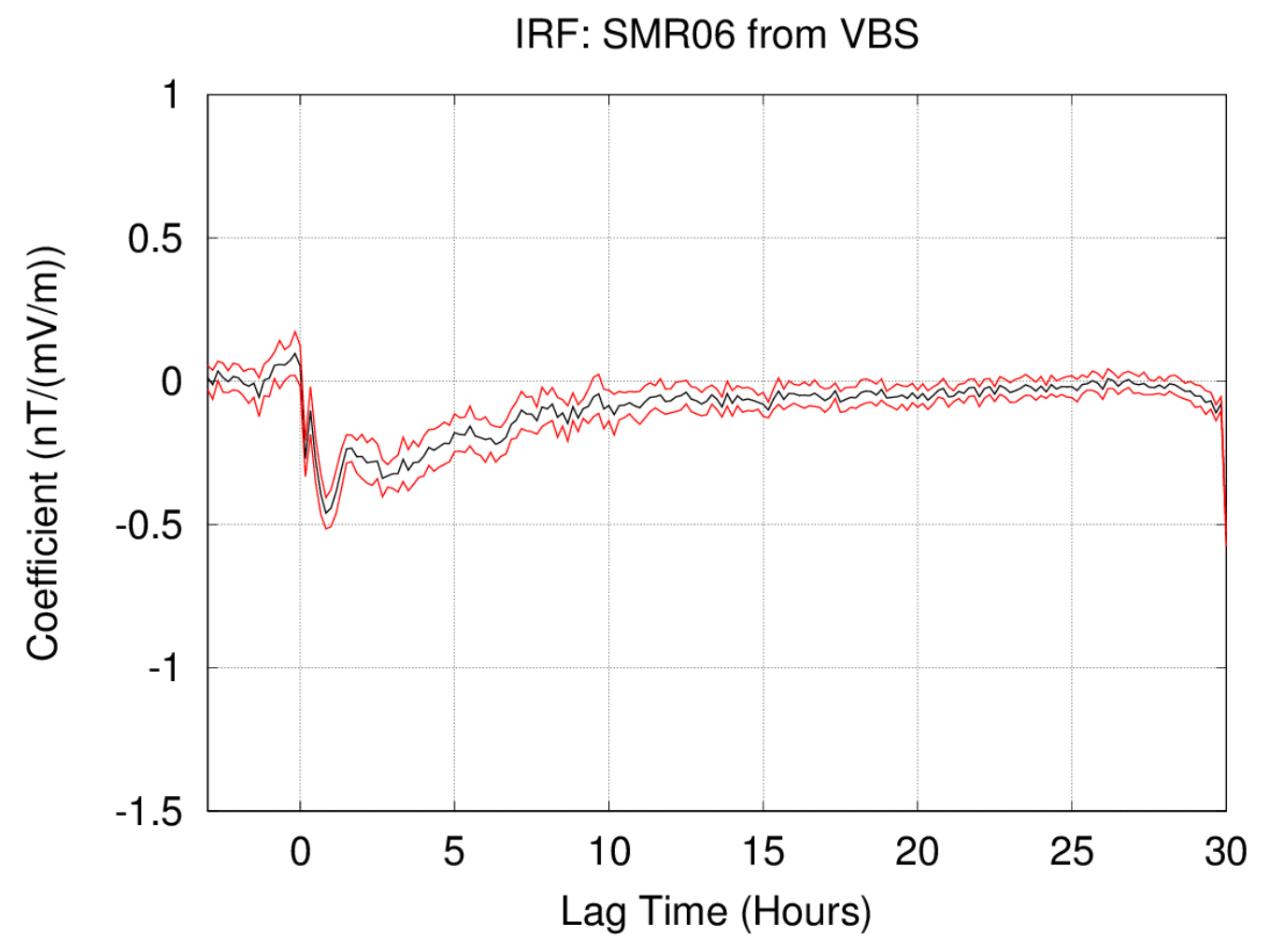

Figure 3.28: The impulse response of dawn sector SMR06 with respect to $\mathrm{v} B_{S}$, where the negative changes in $\mathrm{AL}$ are added as an additional regression variable. 


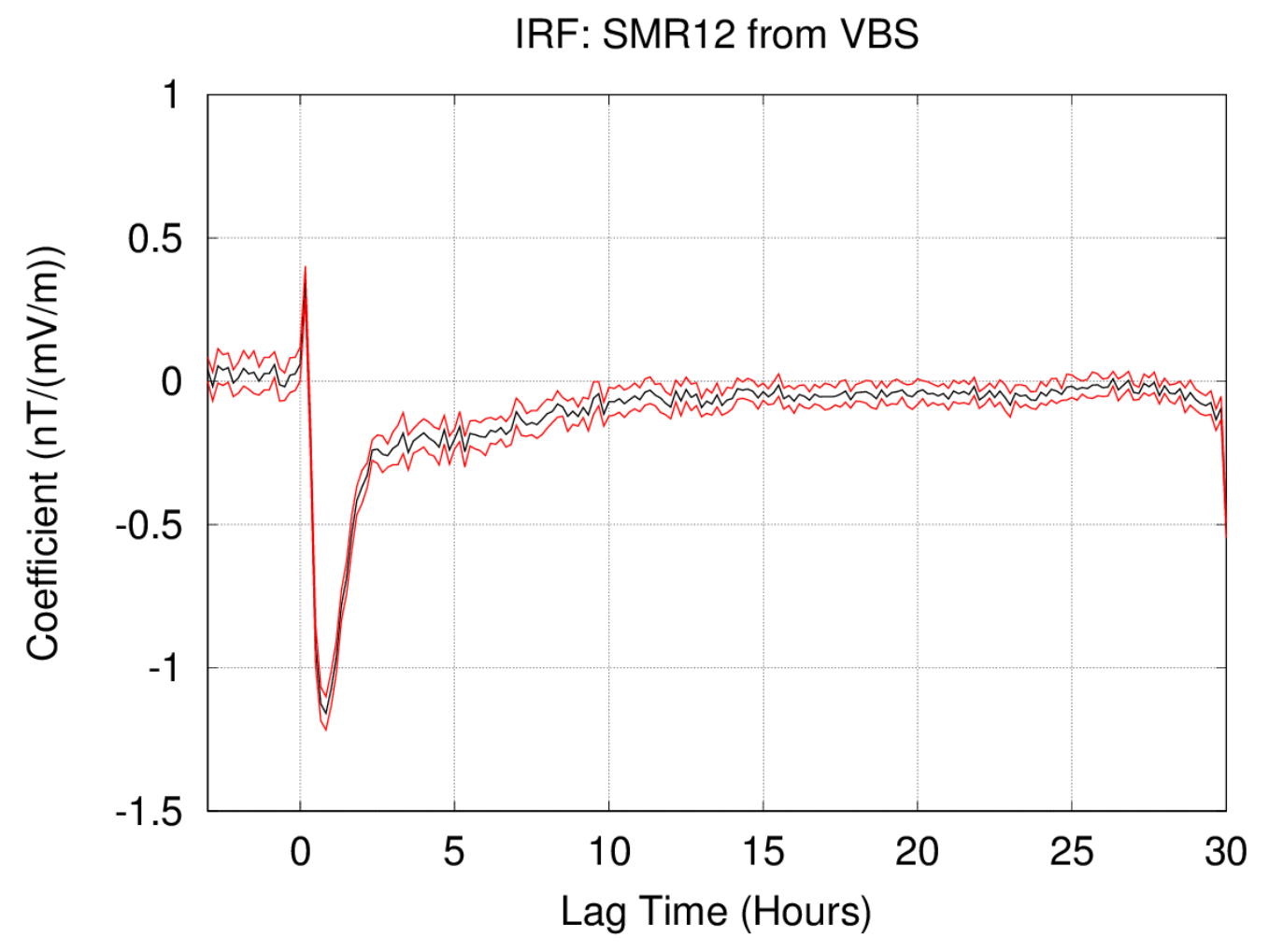

Figure 3.29: The impulse response of noon sector SMR12 with respect to $\mathrm{v} B_{S}$, where the negative changes in $\mathrm{AL}$ are added as an additional regression variable. 


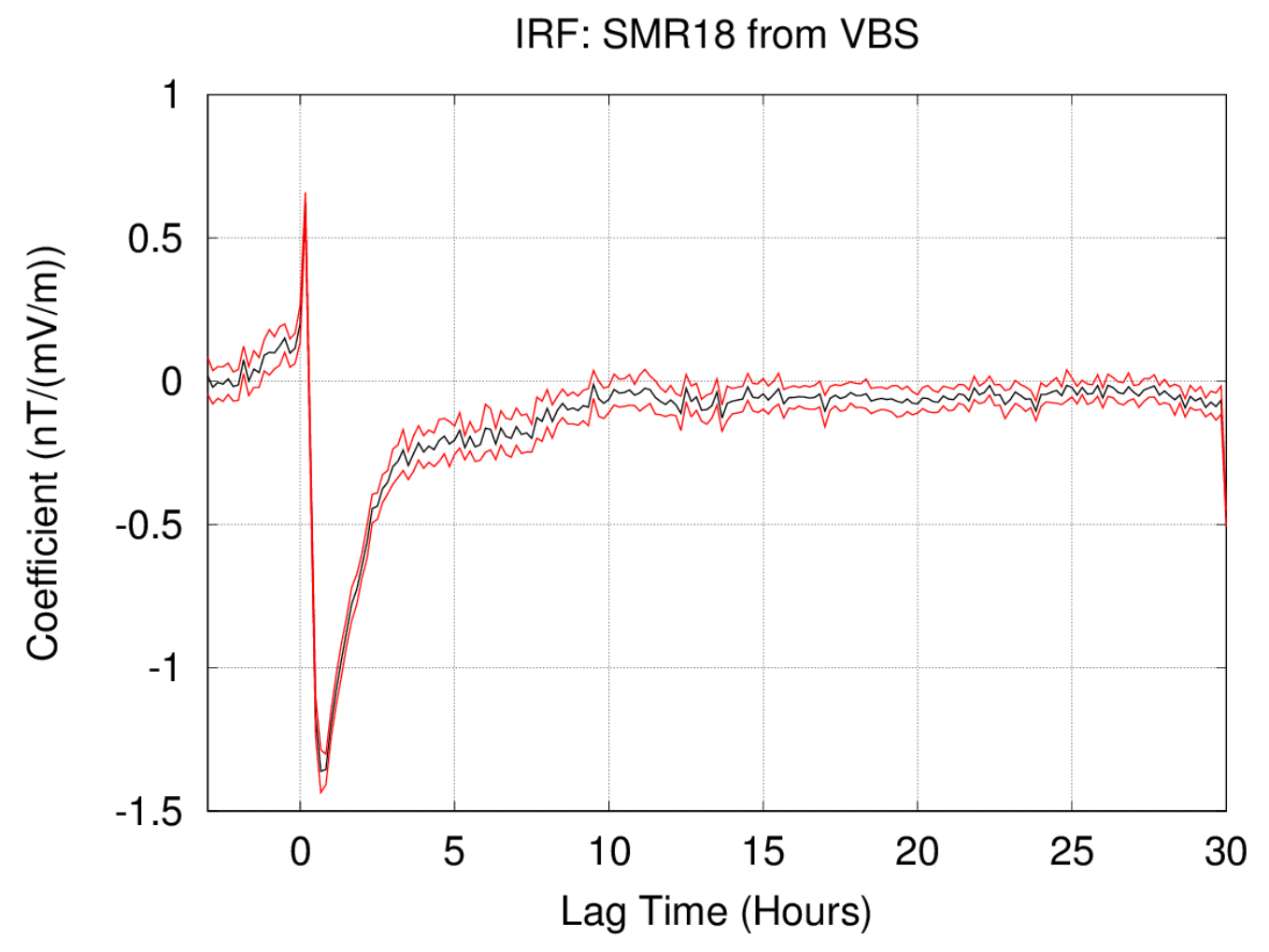

Figure 3.30: The impulse response of dusk sector SMR18 with respect to $\mathrm{v} B_{S}$, where the negative changes in $\mathrm{AL}$ are added as an additional regression variable. 


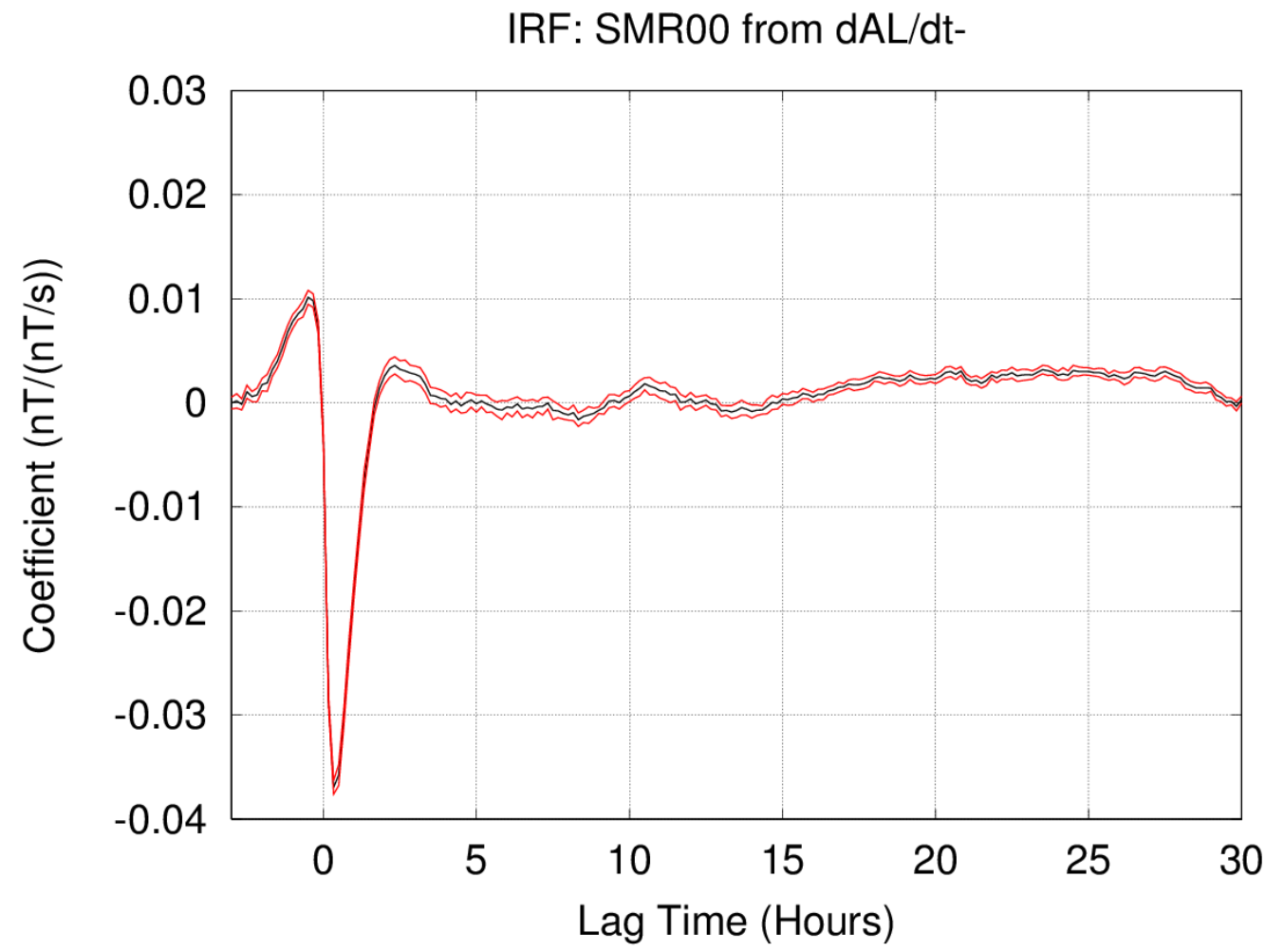

Figure 3.31: The impulse response of midnight sector SMR00 with respect to negative $d(A L) / d t$ to represent the substorm unloading process. A positive (negative) value means the unloading produces a strengthening (weakening) of the index. 


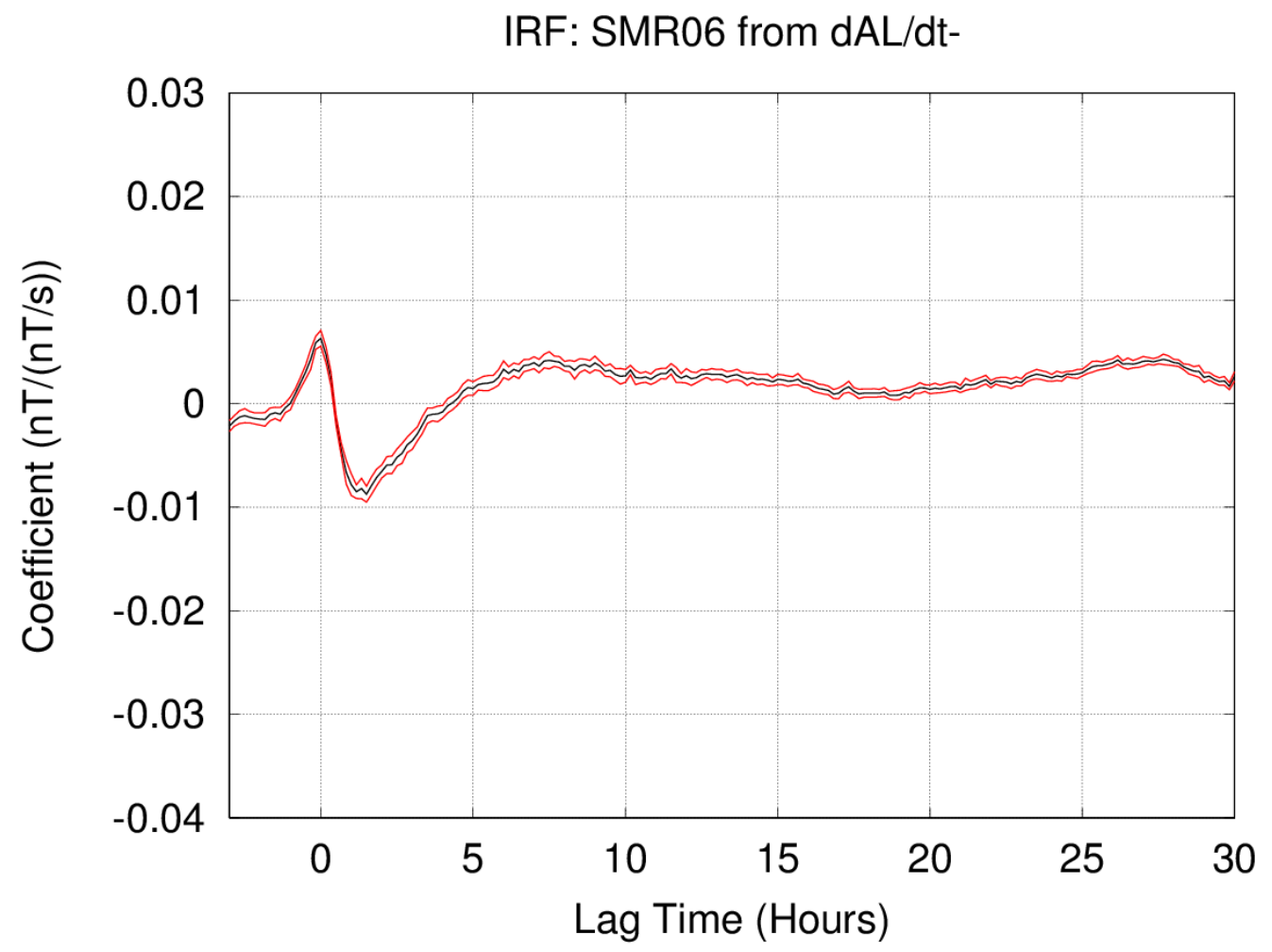

Figure 3.32: The impulse response of dawn sector SMR06 with respect to negative $d(A L) / d t$ to represent the substorm unloading process. A positive (negative) value means the unloading produces a strengthening (weakening) of the index. 


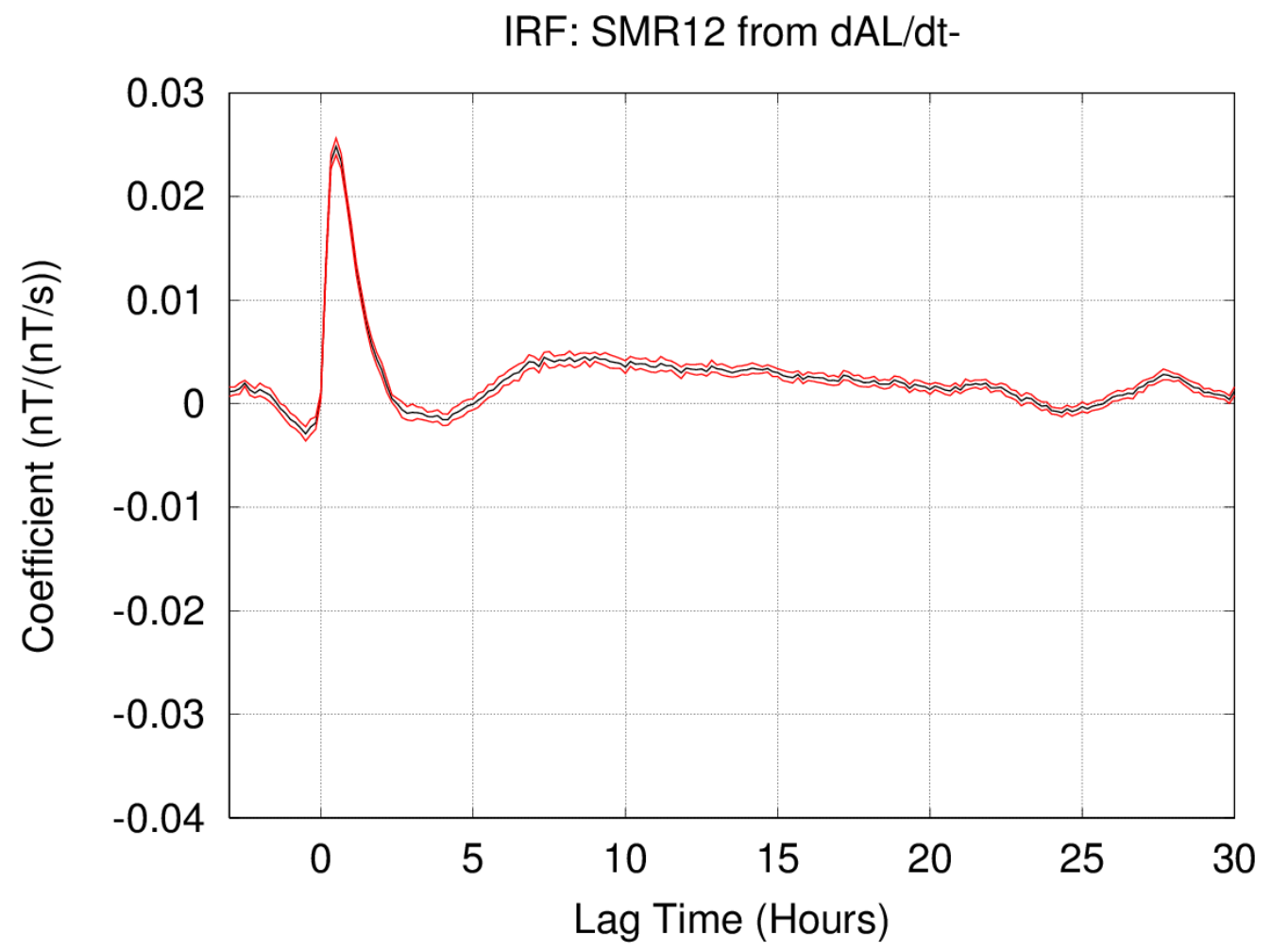

Figure 3.33: The impulse response of noon sector SMR12 with respect to negative $d(A L) / d t$ to represent the substorm unloading process. A positive (negative) value means the unloading produces a strengthening (weakening) of the index. 


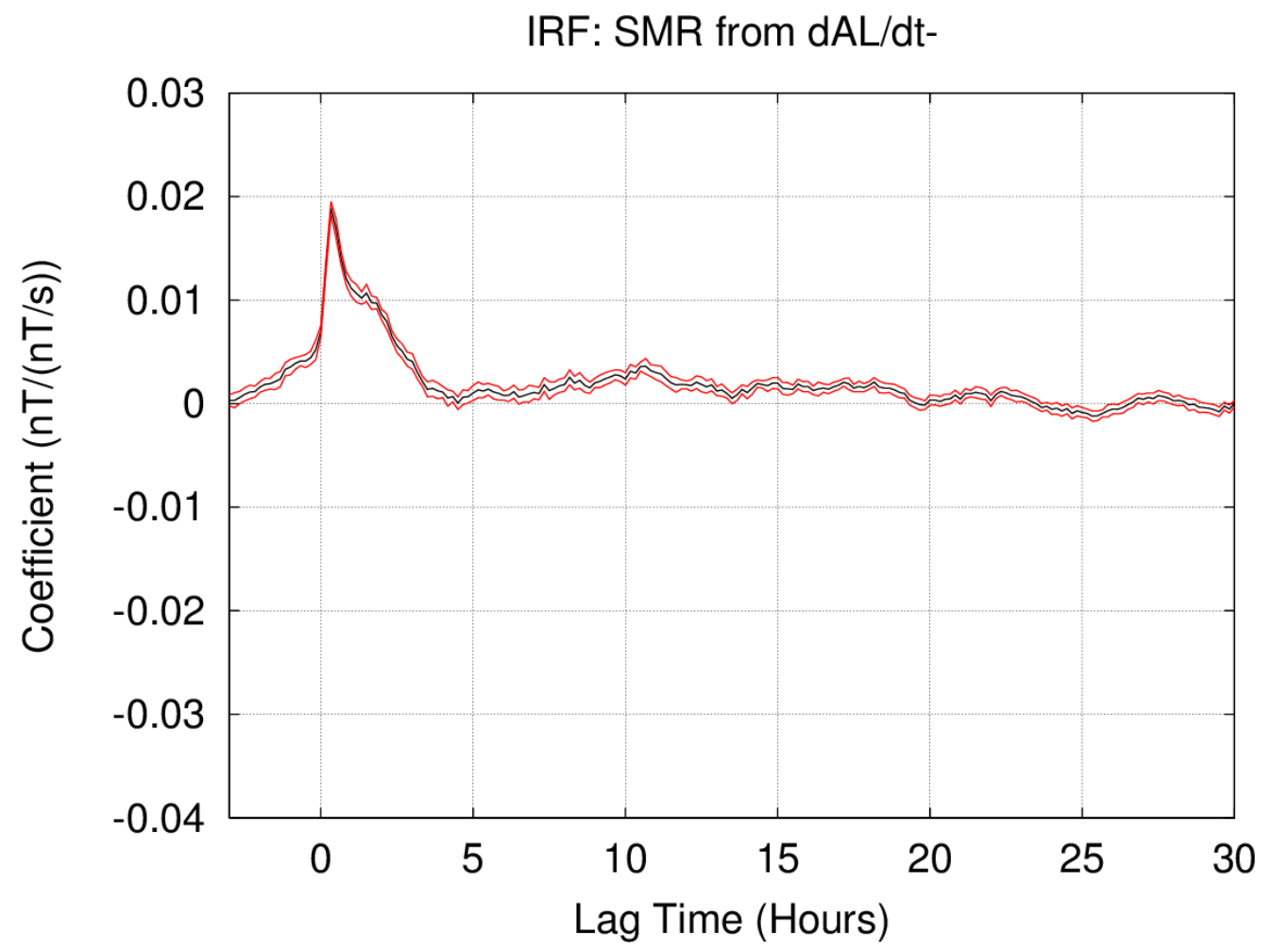

Figure 3.34: The impulse response of dusk sector SMR18 with respect to negative $d(A L) / d t$ to represent the substorm unloading process. A positive (negative) value means the unloading produces a strengthening (weakening) of the index. 


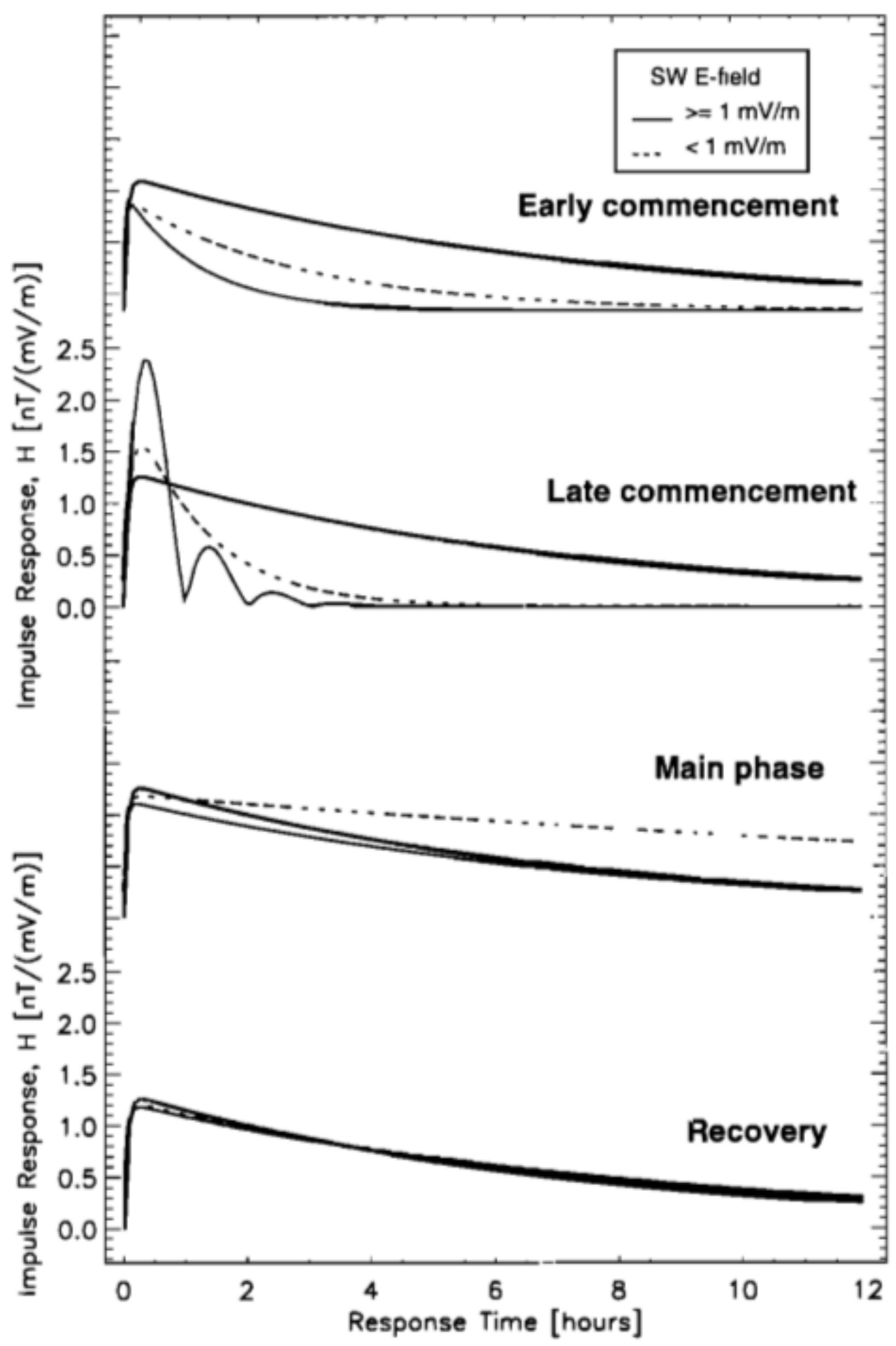

Figure 3.35: The impulse response of modeled $D_{S T}$ separated by storm phase with respect to v $B_{S}$ as found in Vassiliadis et al. (1999). The dashed (solid) lines represent weak (strong) driving. The heavy line is averaged for all conditions. We draw attention to the the strongly driven late commencement case. Could this be the a signature of behavior of midnight and dawn sectors in response to substorm unloading? 


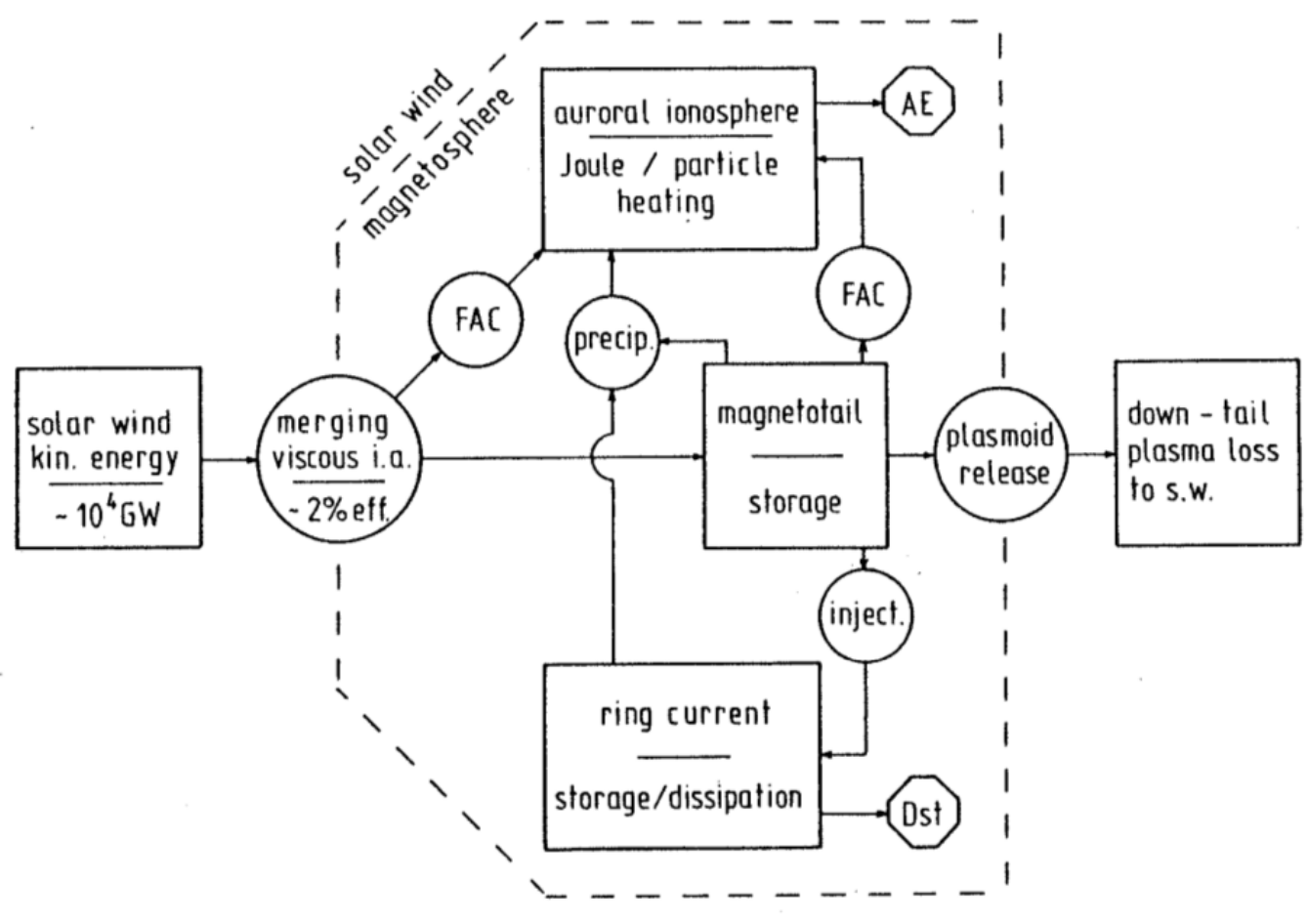

Figure 3.36: This schematic diagram from Baumjohann (1986) shows the conventional understanding of solar wind energy flow and dissipation in the magnetosphere. Note that $D_{S T}$ is considered to be dependent on ring current. 


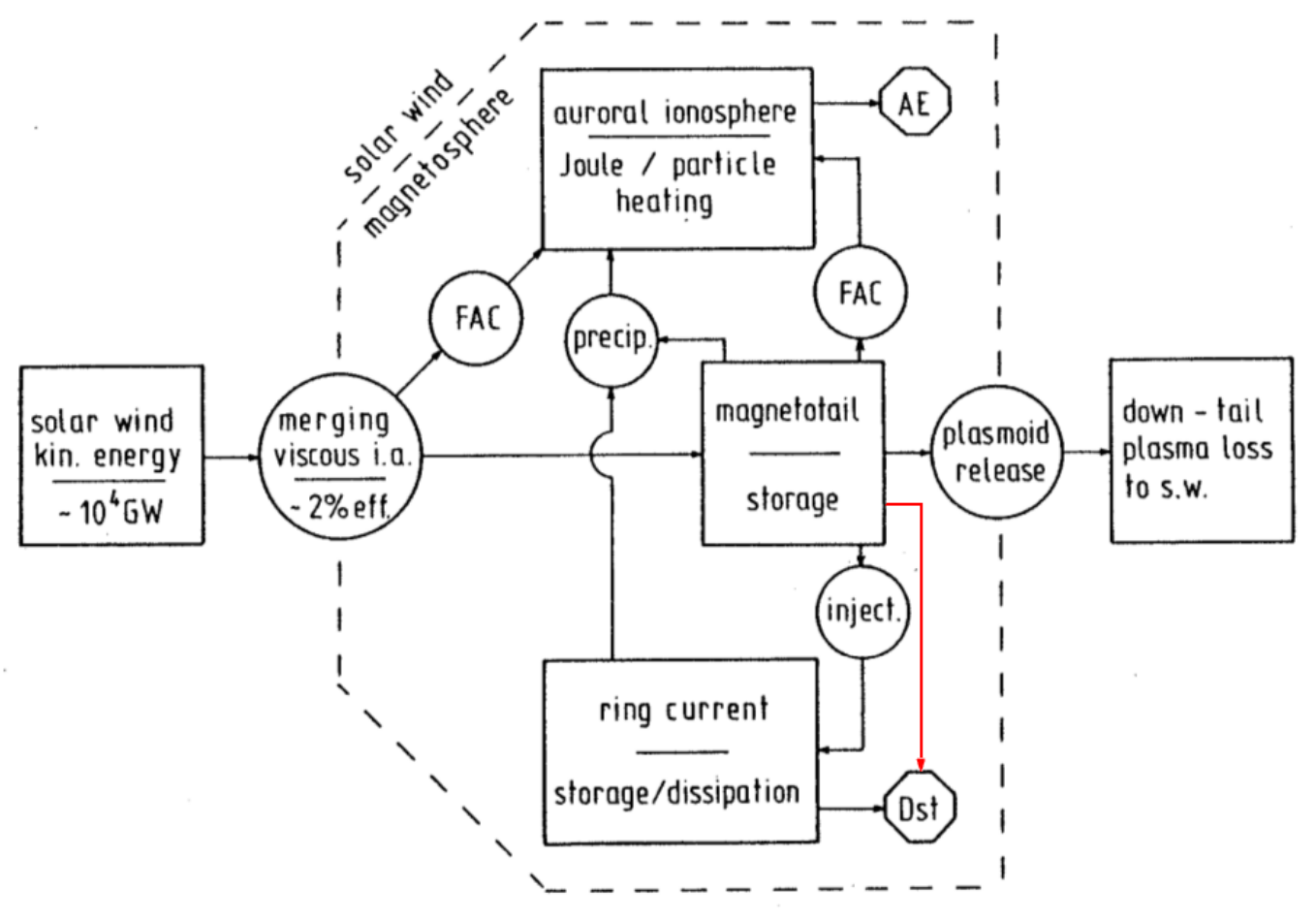

Figure 3.37: This schematic diagram from Baumjohann (1986) of solar wind energy flow and dissipation in the magnetosphere has been modified (in red) to add the effect of cross tail currents. Although several studies have found non-trivial contributions of tail current to storm-time depression, many in the field continue to view $D_{S T}$ and its clones as primarily a ring current proxy as in figure 3.36 . 


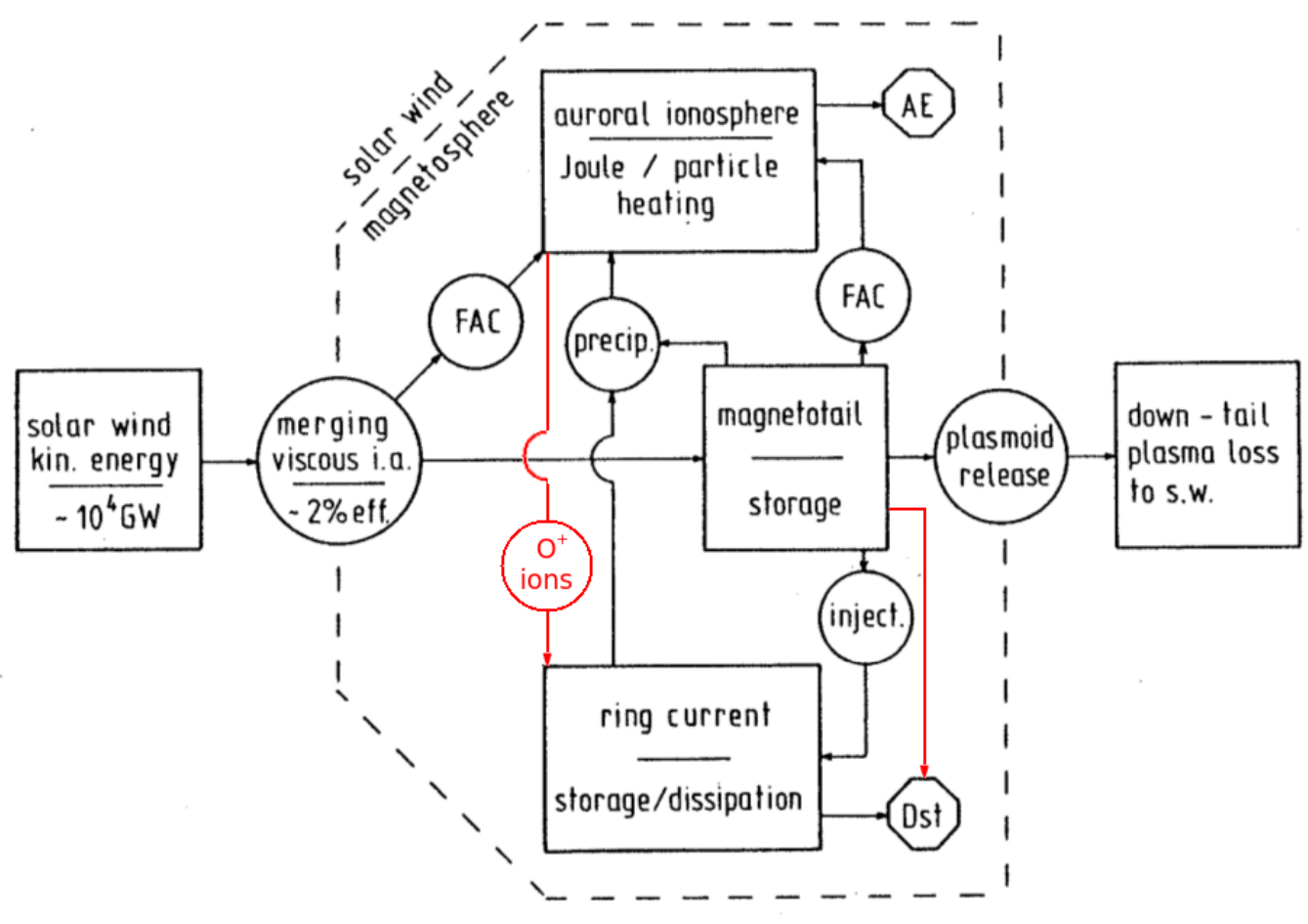

Figure 3.38: This schematic diagram from Baumjohann (1986) of solar wind energy flow and dissipation in the magnetosphere has been further modified (in red) from figure 3.37 to add the time delayed contribution of oxygen ions liberated from the ionosphere by substorm events. In this view, supported by the impulse response studies presented in this chapter, the ring current contribution develops after the initial tail contribution. 


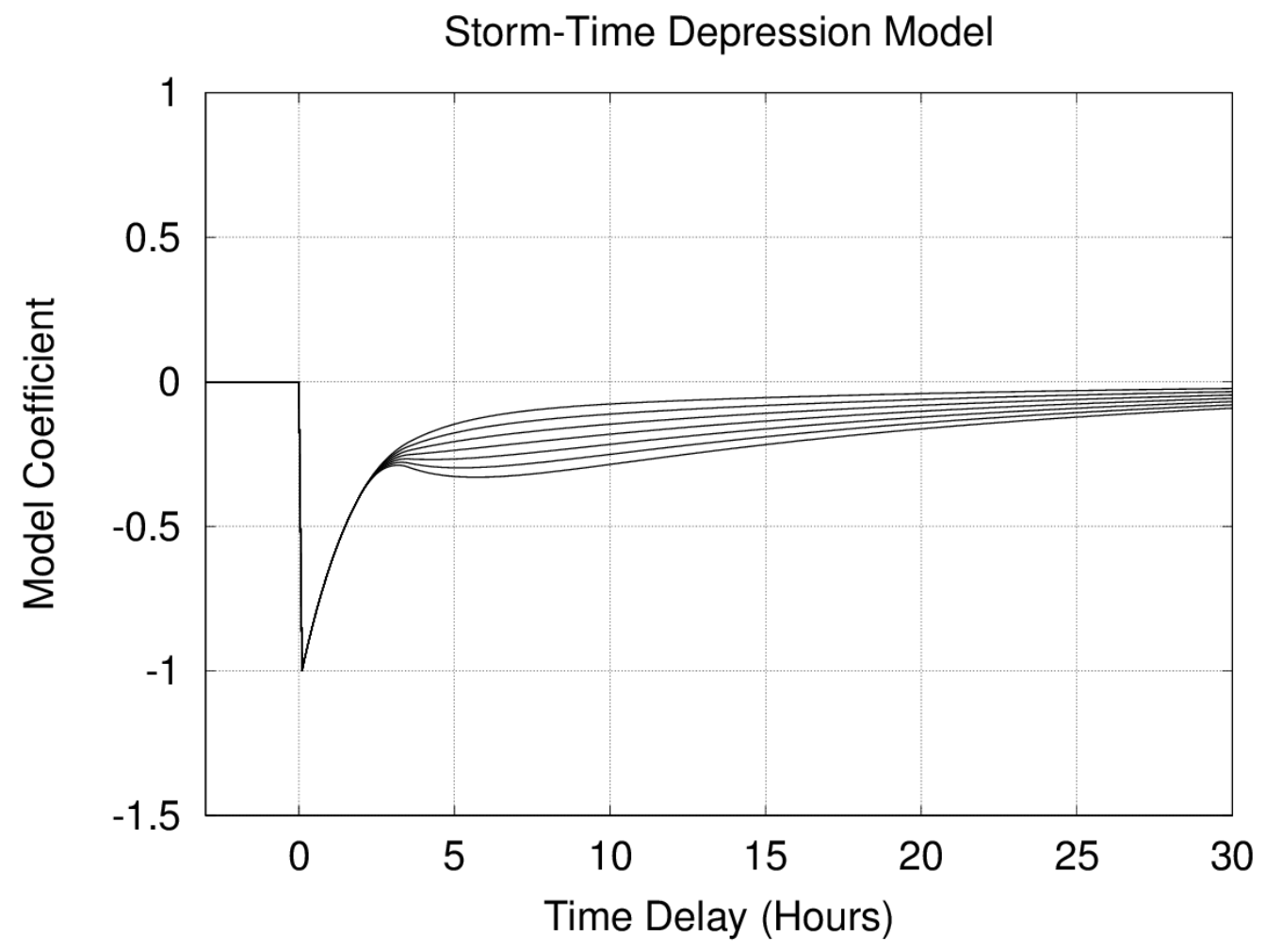

Figure 3.39: An example IRF from the model, schematically shown in the previous figure. This figure was produced as follows. (1) An exponential decay series representing the hypothesized tail current contribution to geomagnetic storm-time depression was produced according to $Y_{1}(t)=$ $-e^{-t / \tau_{1}}$, where $\tau_{1}$ is 2 hours and $\mathrm{t}$ is lag time. (2) The rate of decline in magnitude for $y_{1}$, assumed to be partly due to substorm activity, is $\dot{Y}_{1}=-Y_{1} / \tau_{1}$. This is assumed to be related to $\mathrm{O}+$ ion injection into the ring current, $Q_{O+}=-\kappa \dot{Y}_{1}$. (3) The injected ring current decays at the longer timescale of $\tau_{2}=17.3$ hours, with injection delayed by 3-4.5 hours after the contributing tail current decay, leading to the evolution equation $\Delta Y_{2, t_{m}}=\sum_{n=1}^{N}\left(Q\left(t_{m}-d_{n}\right) / N\right)-Y_{2, t(m-1) / \tau_{2}}$, where $d_{1}=3$ hours and $d_{N}=4.5$ hours. (4) The overall sum, $Y=Y_{1}+Y_{2}$, is plotted versus IRF lag time for values of $\kappa=0.1$ (minimal 2nd development) to $\kappa=0.4$ (prominent 2nd development) in increments of 0.05 . 


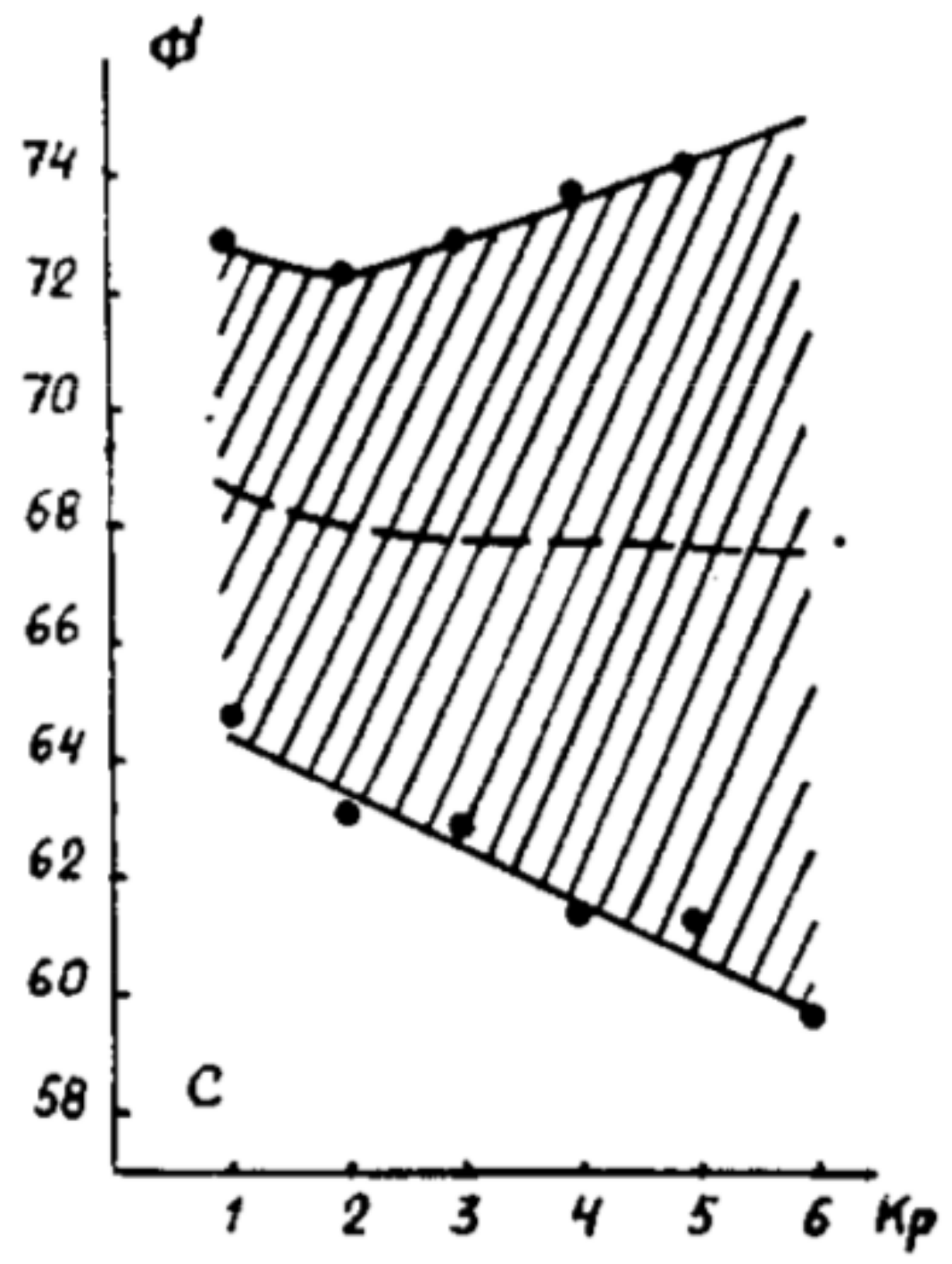

Figure 4.1: The average latitudinal range of the auroral oval, as determined from all-sky-imager cameras, near local midnight parametrized by Kp is shown. It is seen that the equatorward location moves to lower latitudes as activity increases. Feldstein and Starkov (1967) 


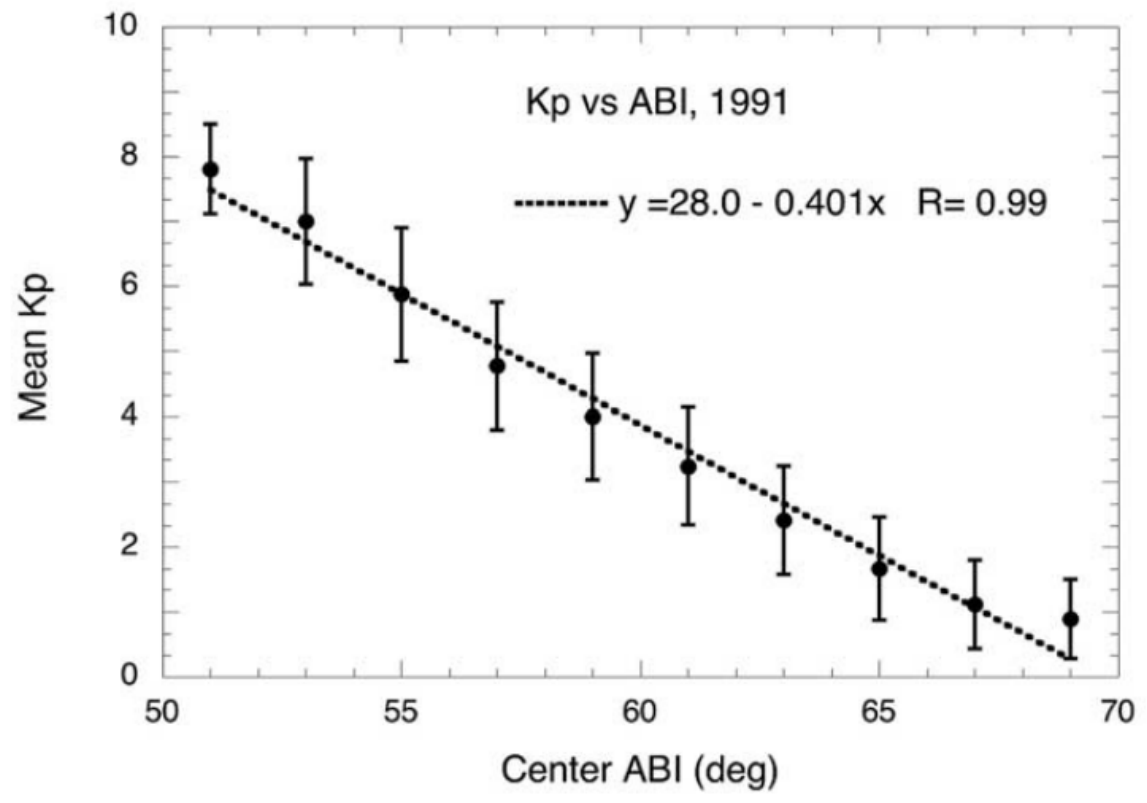

Figure 4.2: An example from Thomsen (2004) showing that the quasi-logarithmic Kp index closely relates to $\mathrm{ABI}$, which tracks the measured latitude of the equatorward auroral boundary as determined from electron precipitation. 


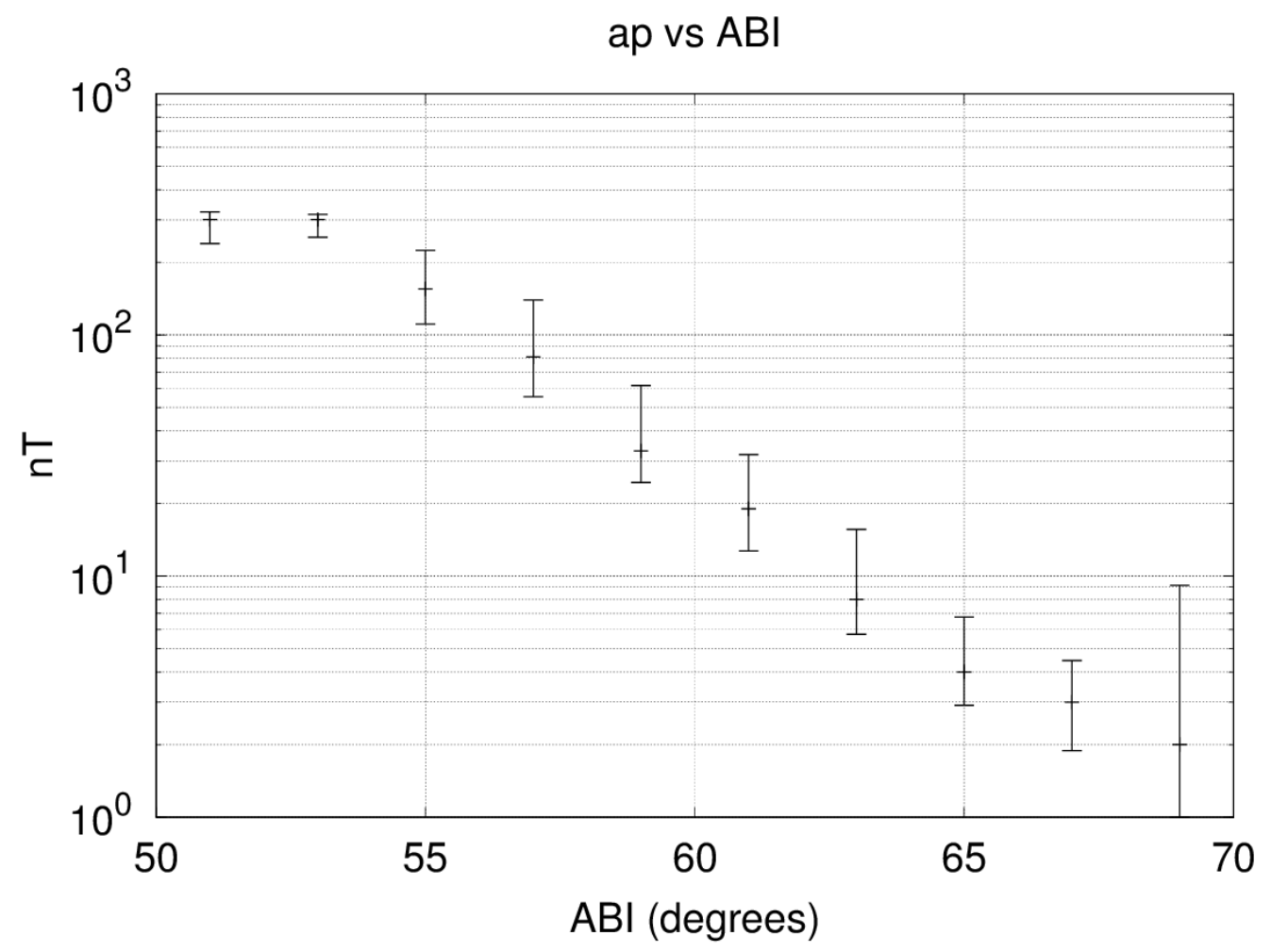

Figure 4.3: The ap index (linear equivalent to the quasi-logarithmic Kp index) plus one is shown on a logarithmic scale versus ABI for years 2000-2009. For robustness, median values are plotted with mean deviation from the median, computed separately for values above and below the median, used for error bars. 


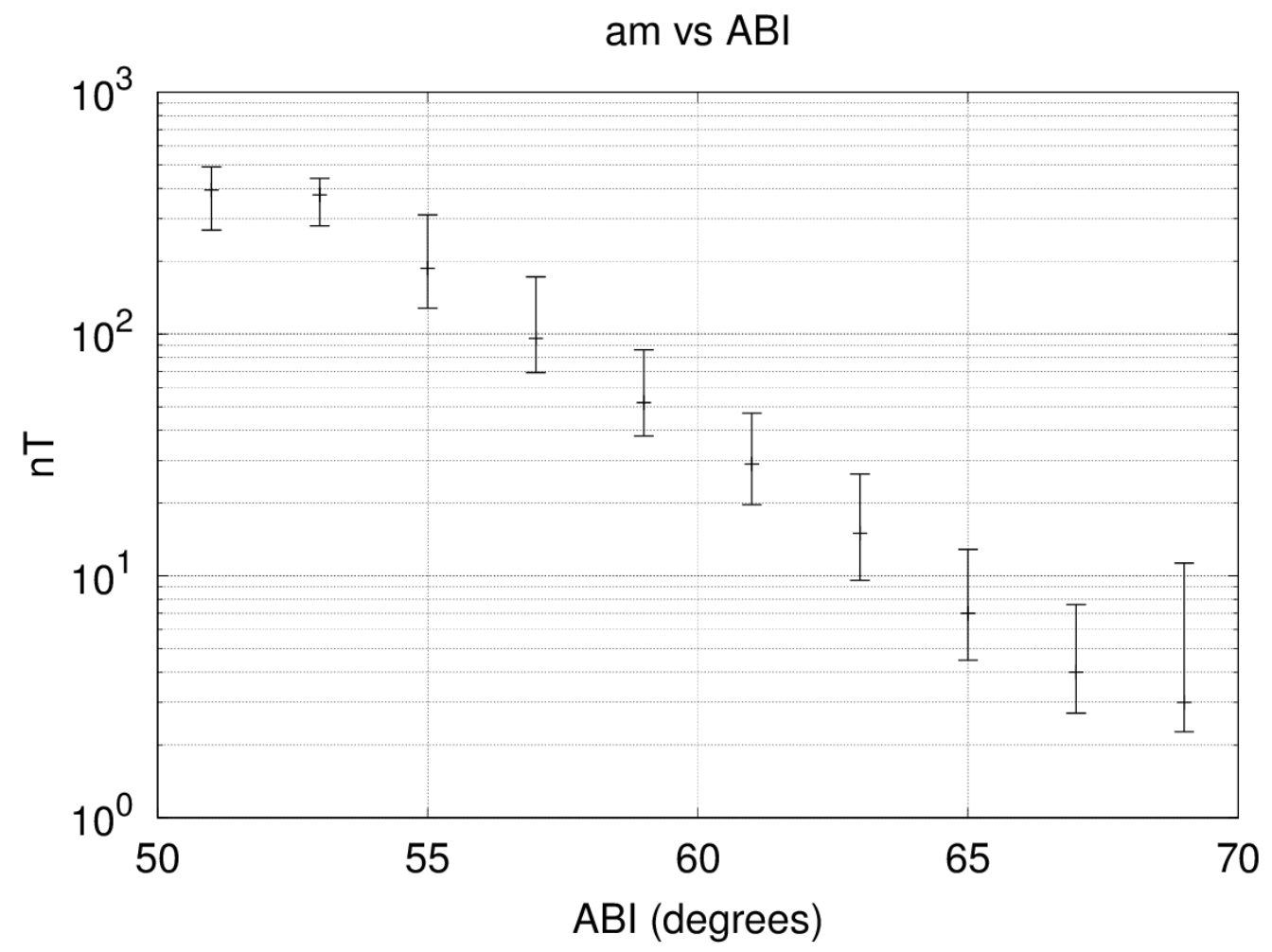

Figure 4.4: The am index (similar to ap, but based on a more global set of observatories and continuous in magnitude) plus one is shown on a logarithmic scale versus ABI for years 2000-2009. Note that the logarithm of am appears more linear with respect to ABI on this data set, confirming claims that am is a higher quality index than Kp. For robustness, median values are plotted with mean deviation from the median, computed separately for values above and below the median, used for error bars. 


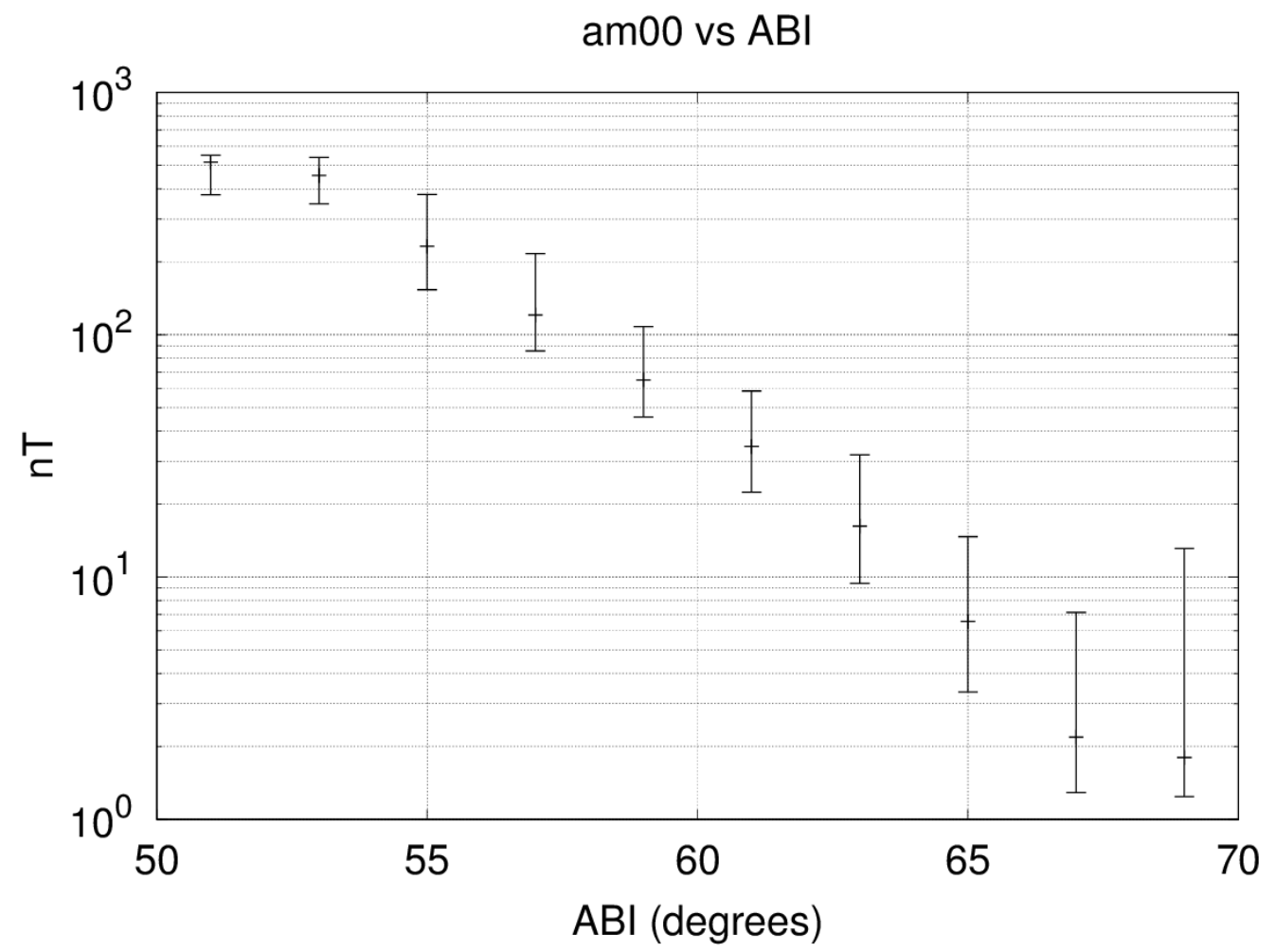

Figure 4.5: The midnight sector am00 index plus one is shown on a logarithmic scale versus ABI for years 2000-2009. For robustness, median values are plotted with mean deviation from the median, computed separately for values above and below the median, used for error bars. 


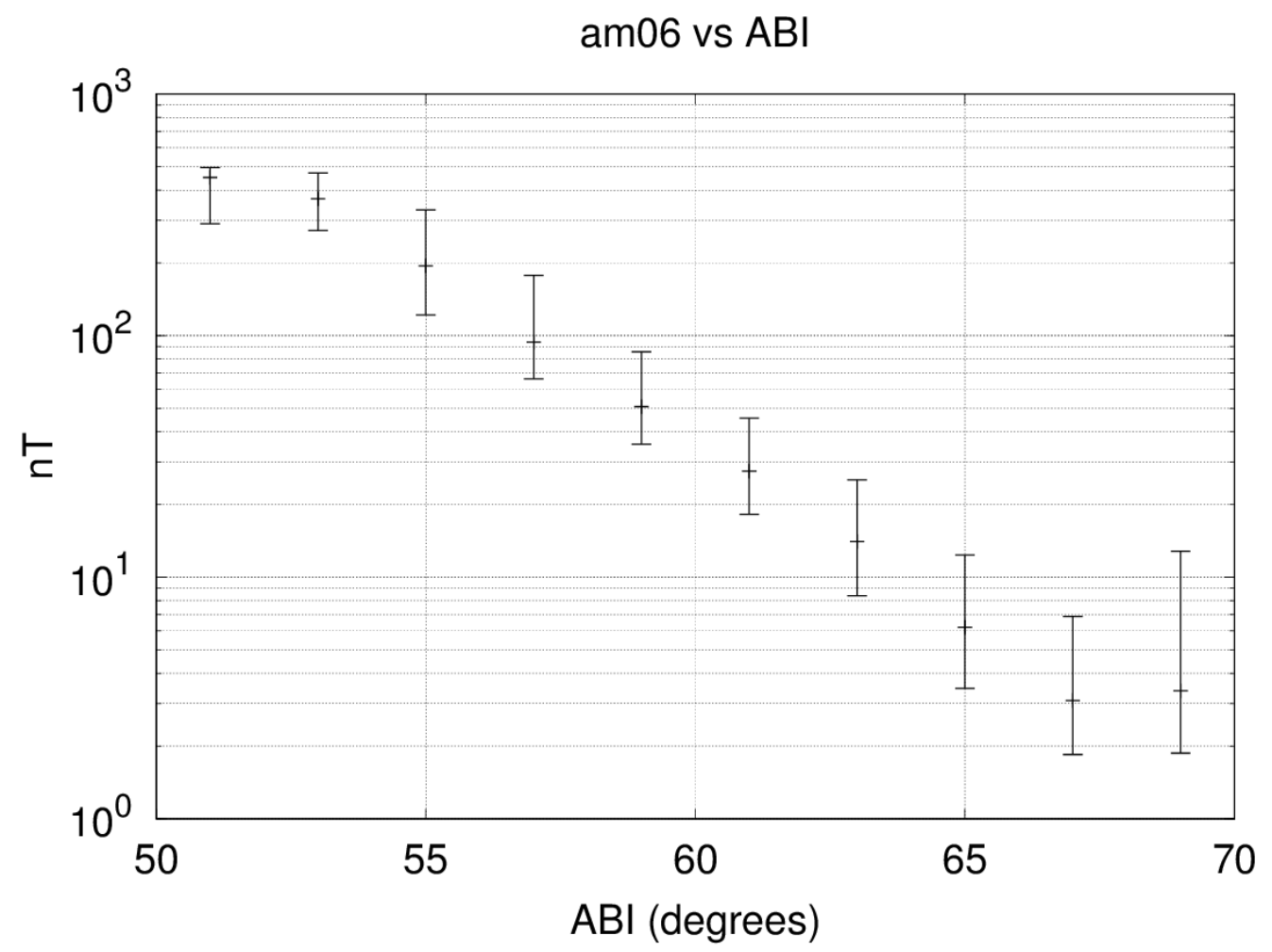

Figure 4.6: The dawn sector am06 index plus one is shown on a logarithmic scale versus ABI for years 2000-2009. For robustness, median values are plotted with mean deviation from the median, computed separately for values above and below the median, used for error bars. 


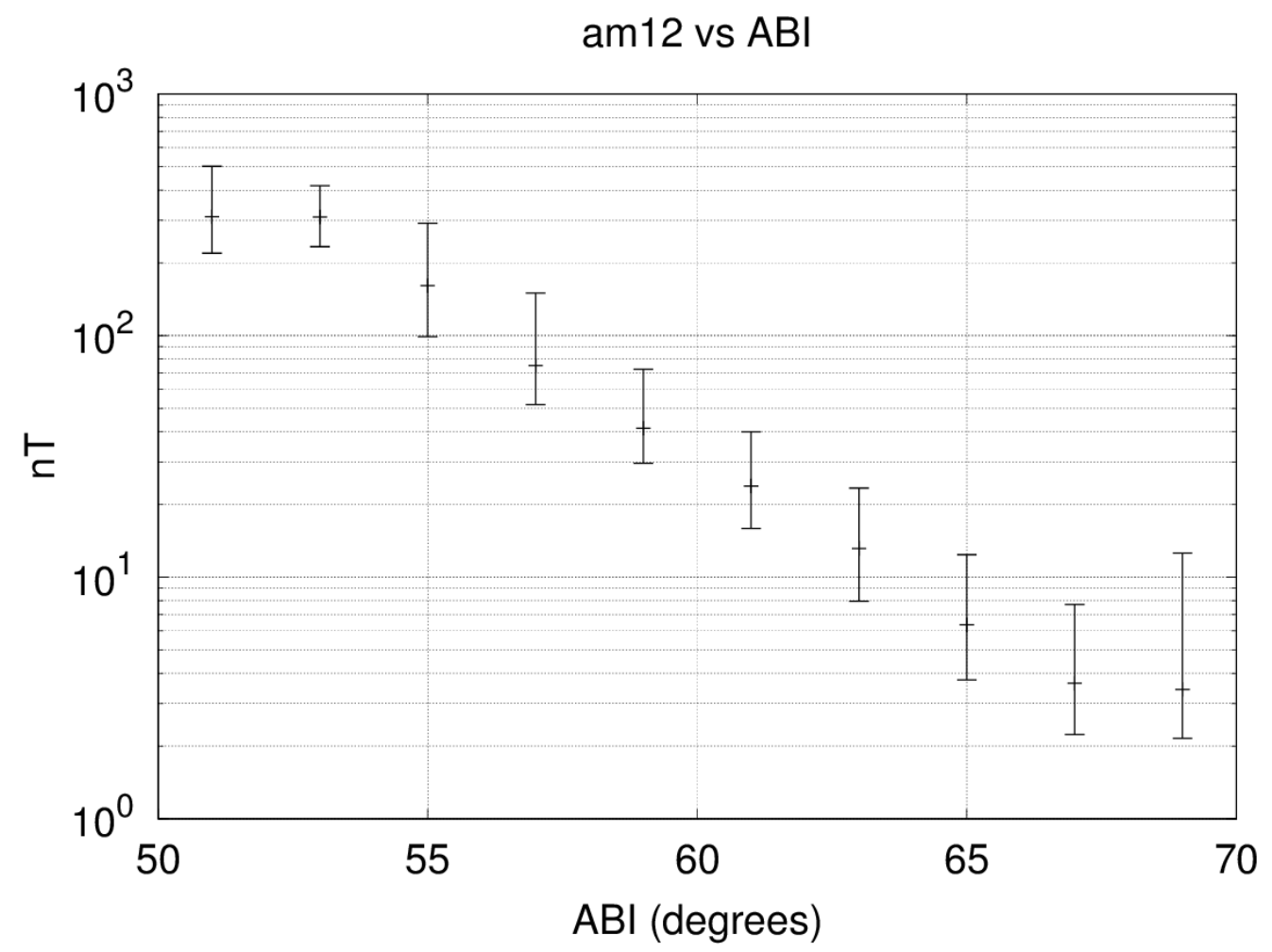

Figure 4.7: The noon sector am12 index plus one is shown on a logarithmic scale versus ABI for years 2000-2009. For robustness, median values are plotted with mean deviation from the median, computed separately for values above and below the median, used for error bars. 


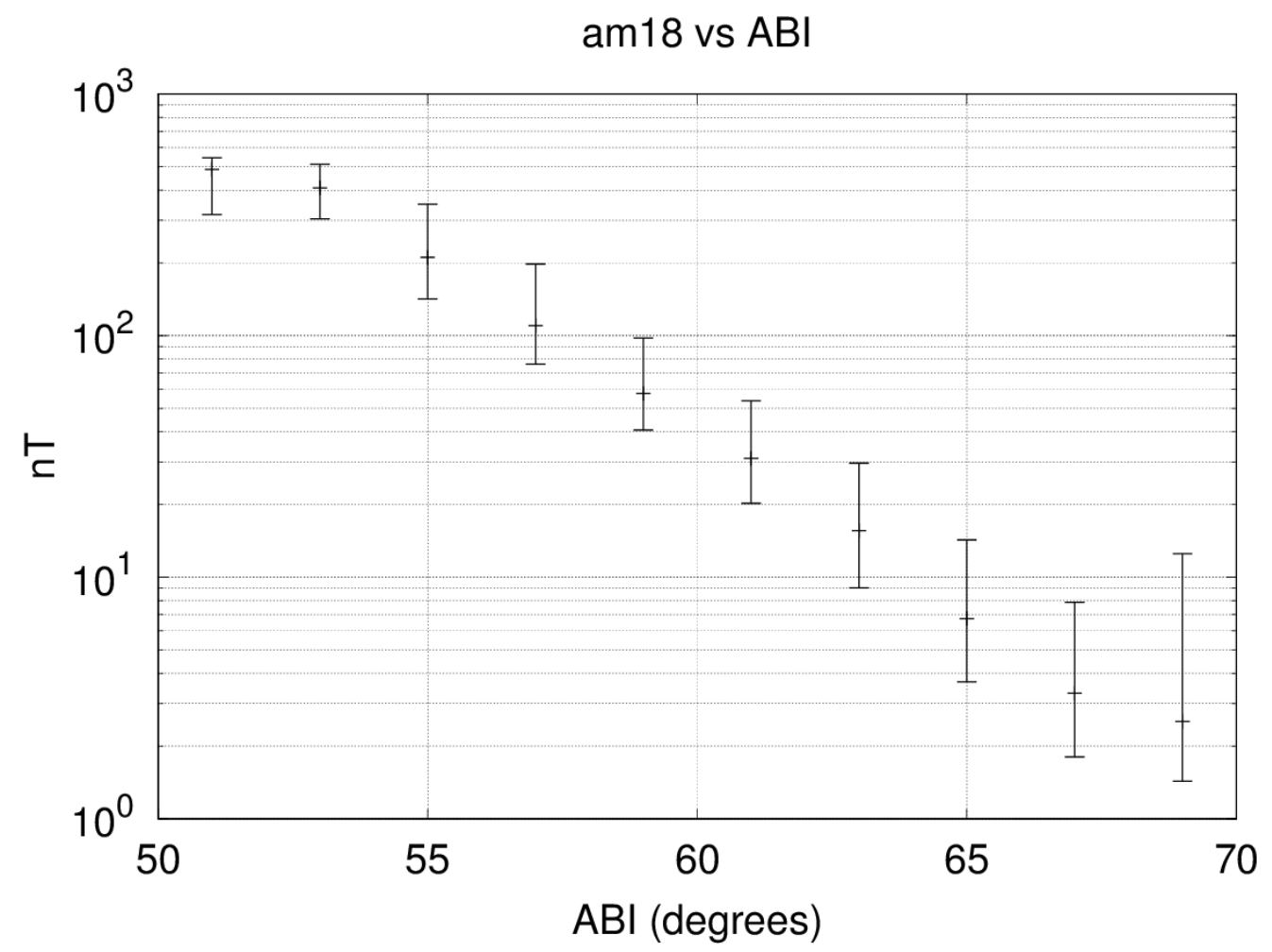

Figure 4.8: The dusk sector am18 index plus one is shown on a logarithmic scale versus ABI for years 2000-2009. For robustness, median values are plotted with mean deviation from the median, computed separately for values above and below the median, used for error bars. 


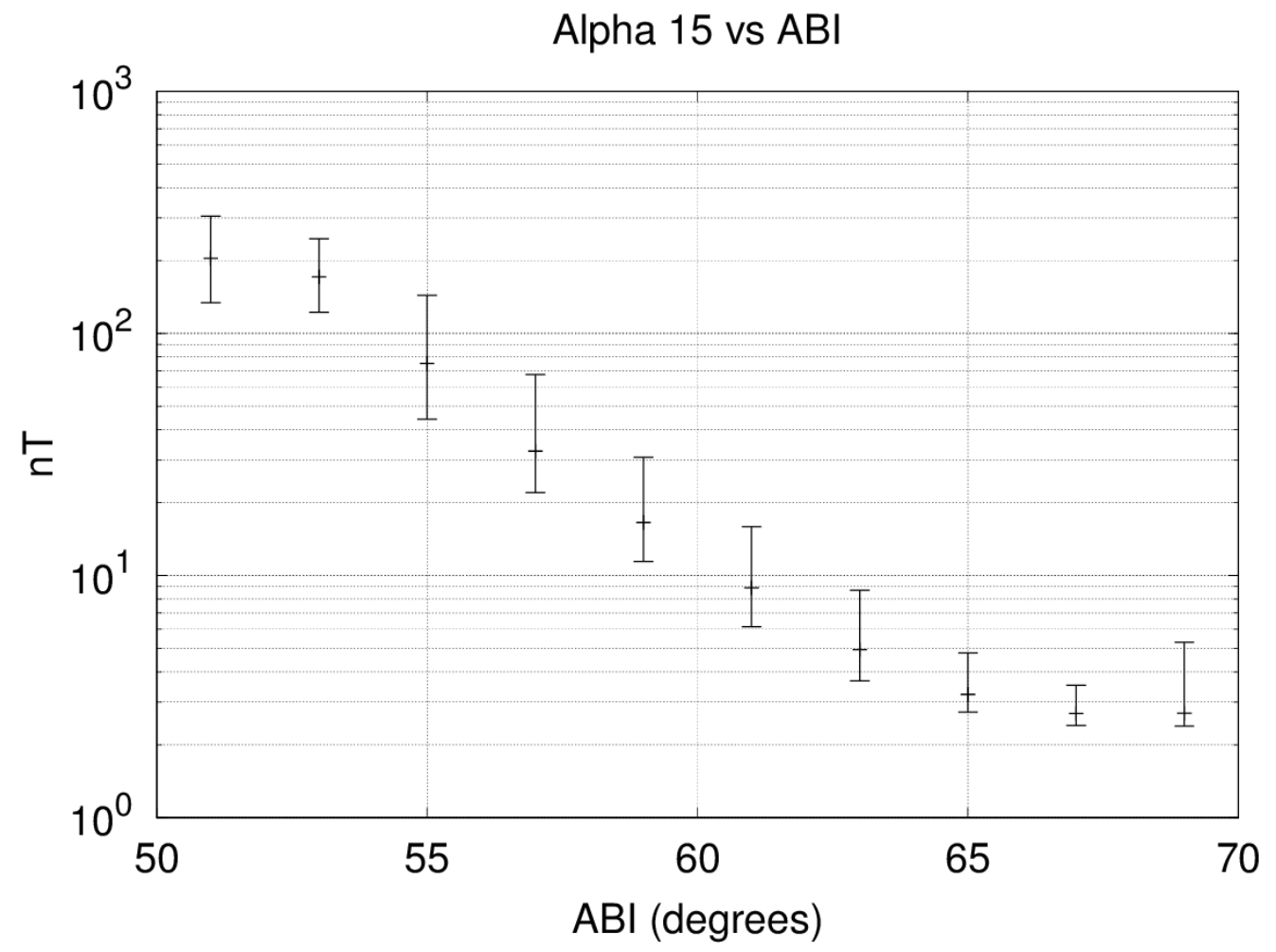

Figure 4.9: The $\alpha 15$ index (similar to am, but available for 15 minute time windows instead of the typical 3 hour windows use for the K-family of indices) plus one is shown on a logarithmic scale versus ABI for years 2000-2009. For robustness, median values are plotted with mean deviation from the median, computed separately for values above and below the median, used for error bars. 


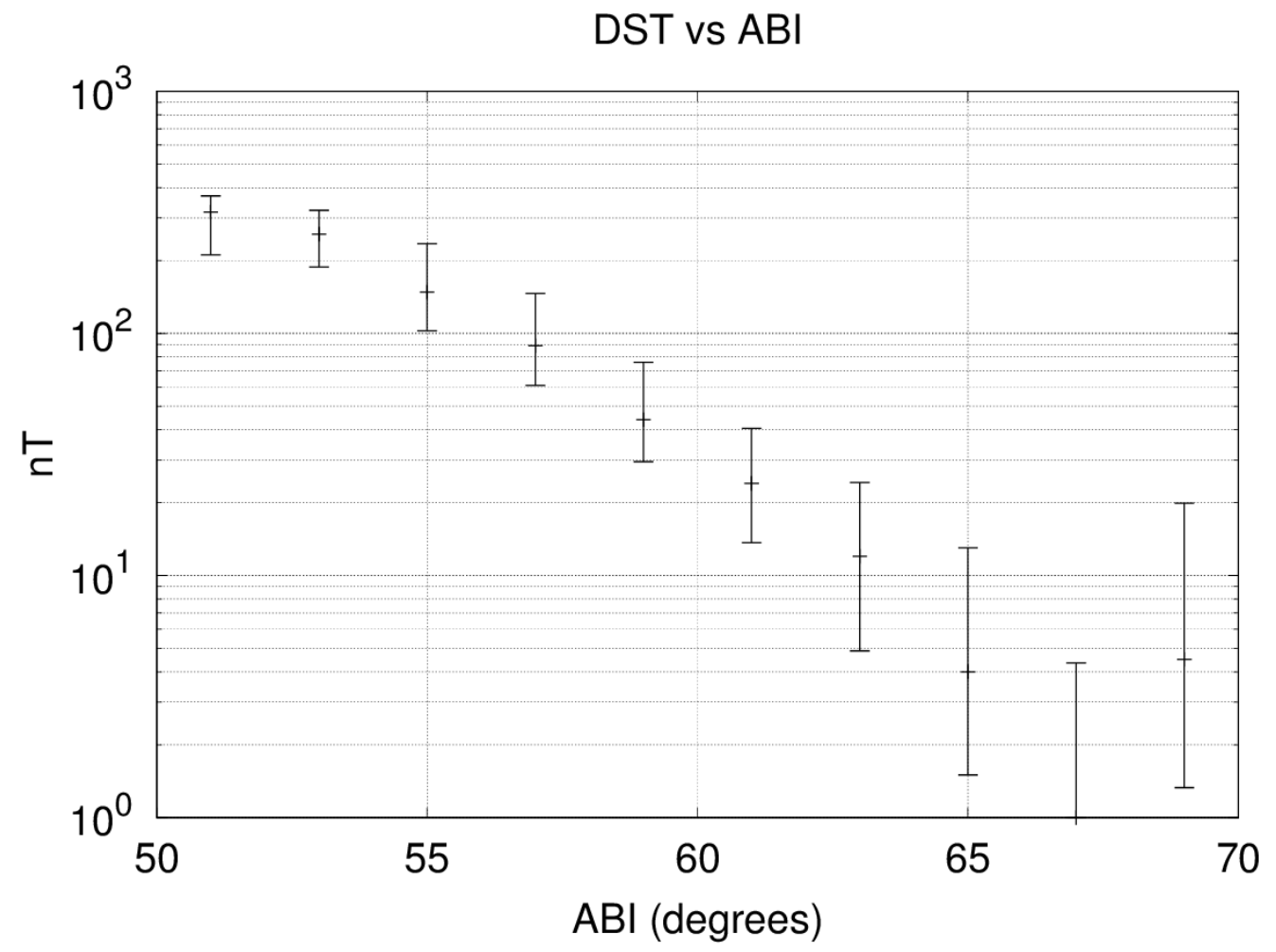

Figure 4.10: The magnitude of $D_{S T}$ index, for times when $D_{S T}<0$, plus one is shown on a logarithmic scale versus ABI for years 2000-2009. For robustness, median values are plotted with mean deviation from the median, computed separately for values above and below the median, used for error bars. Note that $D_{S T}$ is mostly linear on this scaling, as are the K-family indices. 


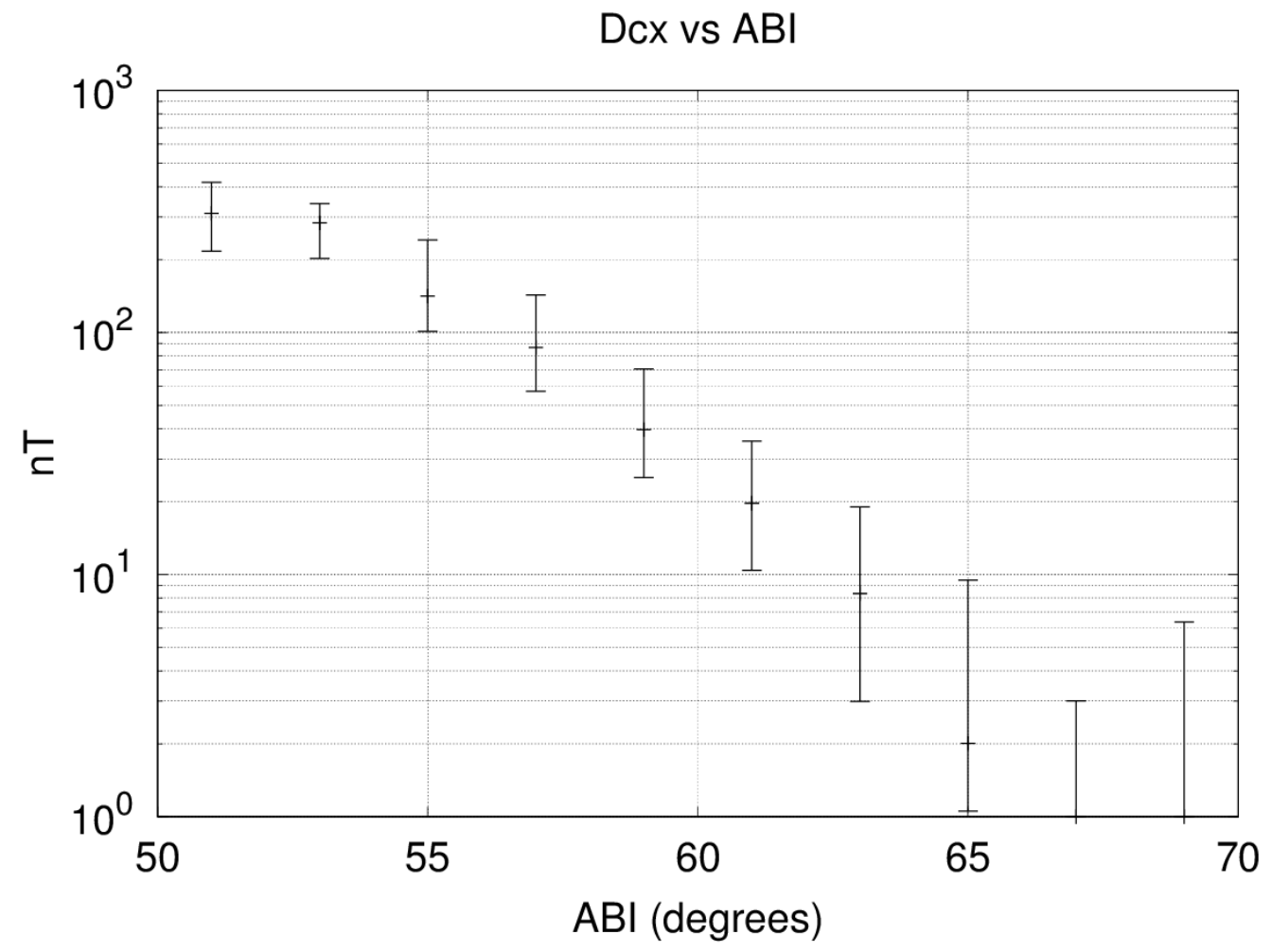

Figure 4.11: The magnitude of Dcx index, for times when $D c x<0$, plus one is shown on a logarithmic scale versus ABI for years 2000-2009. For robustness, median values are plotted with mean deviation from the median, computed separately for values above and below the median, used for error bars. Note that Dcx is more linear on this scaling than $D_{S T}$, giving support to claims that Dcx is a corrected version of $D_{S T}$. 


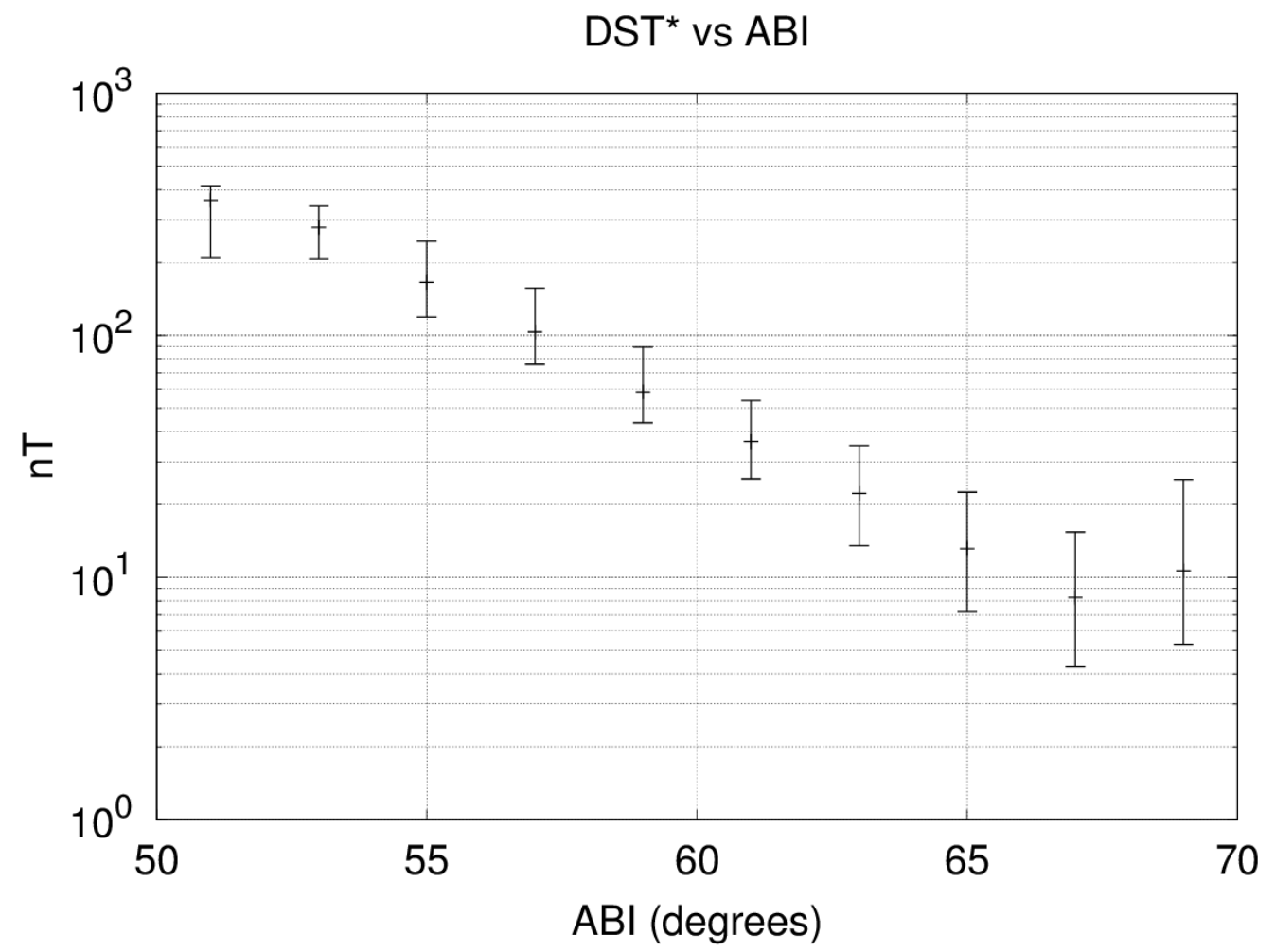

Figure 4.12: The magnitude of pressure corrected $D_{S T}^{*}$ index, for times when $D_{S T}^{*}<0$, plus one is shown on a logarithmic scale versus ABI for years 2000-2009. For robustness, median values are plotted with mean deviation from the median, computed separately for values above and below the median, used for error bars. Note that $D_{S T}$ is mostly linear on this scaling, as are the K-family indices. 


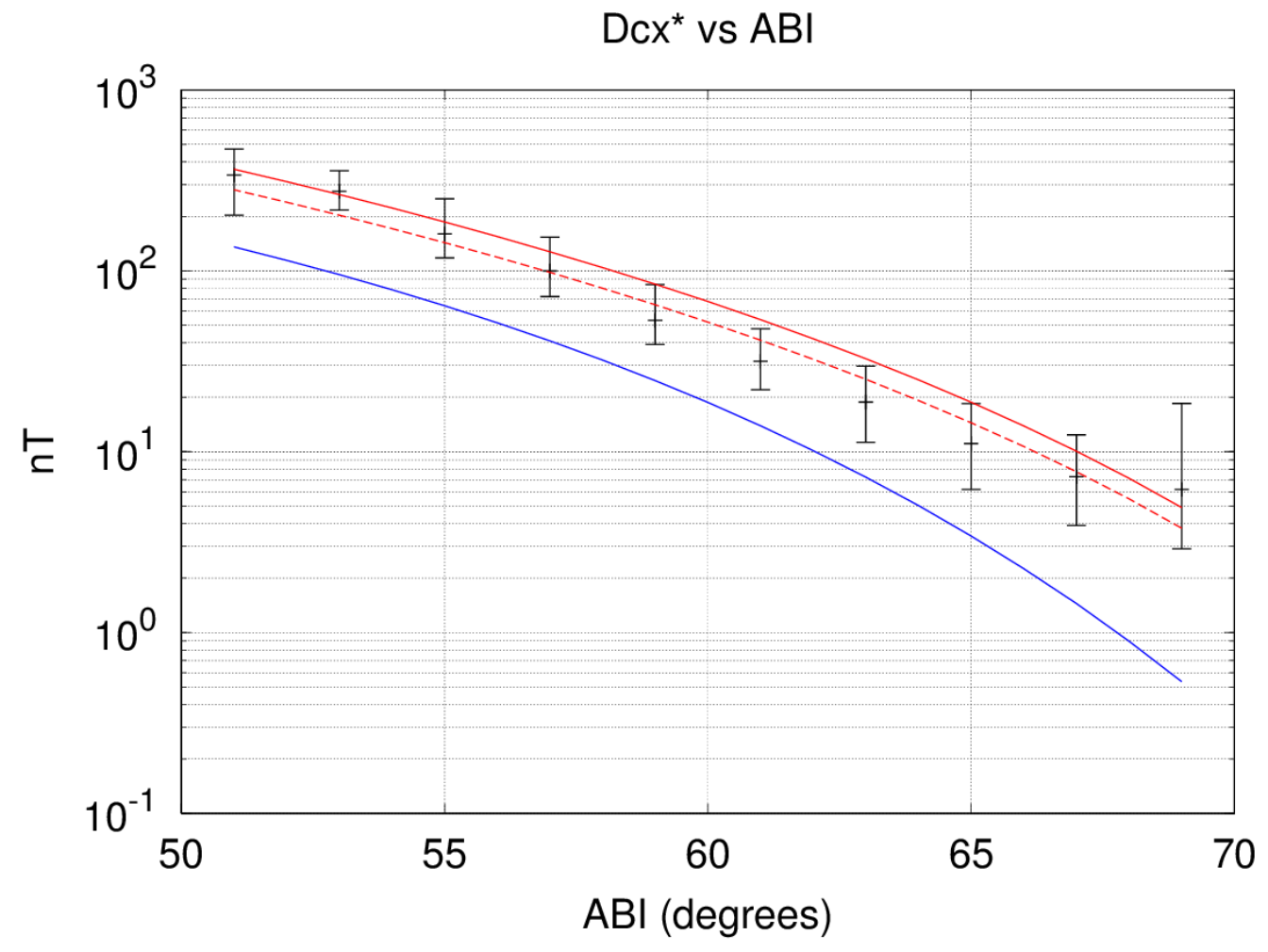

Figure 4.13: The magnitude of pressure corrected Dcx* index, for times when $D c x *<0$, plus one is shown on a logarithmic scale versus ABI for years 2000-2009. For robustness, median values are plotted with mean deviation from the median, computed separately for values above and below the median, used for error bars. Note that Dcx is more linear on this scaling than $D_{S T}$, giving support to claims that Dcx is a corrected version of $D_{S T}$. The theoretical tail contribution of tail currents derived in Maltsev (2004) is shown in red, with solid and dashed lines for cases with and without a ground induction factor of 1.3, respectively. The empirically obtained formula for tail current contribution according to Asikainen et al. (2010) is shown in blue. 


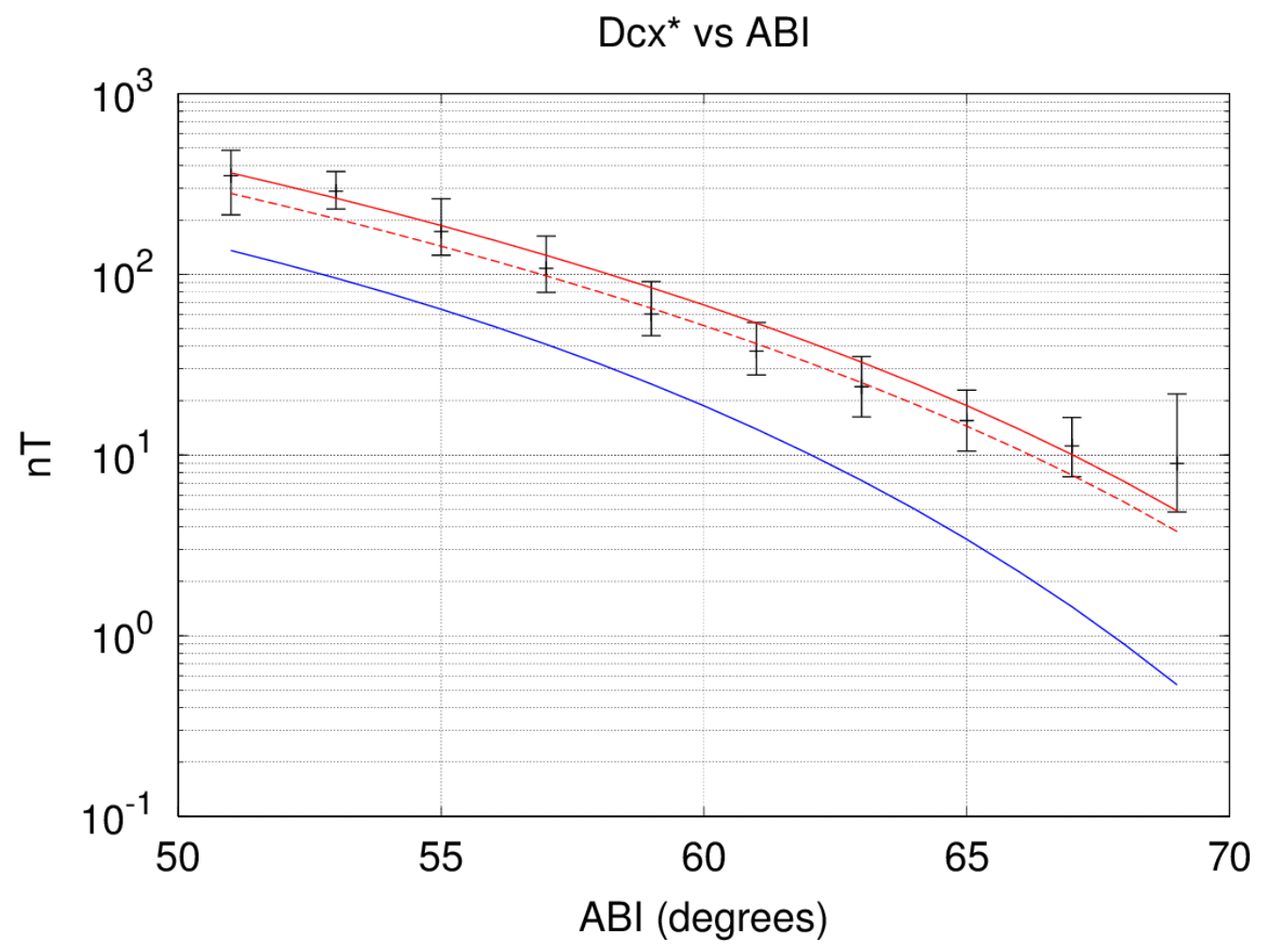

Figure 4.14: The magnitude of pressure corrected Dcx* index, using the Dcx specific pressure correction obtained in Asikainen et al. (2010), for times when $D c x *<0$, plus one is shown on a logarithmic scale versus ABI for years 2000-2009. For robustness, median values are plotted with mean deviation from the median, computed separately for values above and below the median, used for error bars. Note that Dcx is more linear on this scaling than $D_{S T}$, giving support to claims that Dcx is a corrected version of $D_{S T}$. The theoretical tail contribution of tail currents derived in Maltsev (2004) is shown in red, with solid and dashed lines for cases with and without a ground induction factor of 1.3, respectively. The empirically obtained formula for tail current contribution according to Asikainen et al. (2010) is shown in blue. 


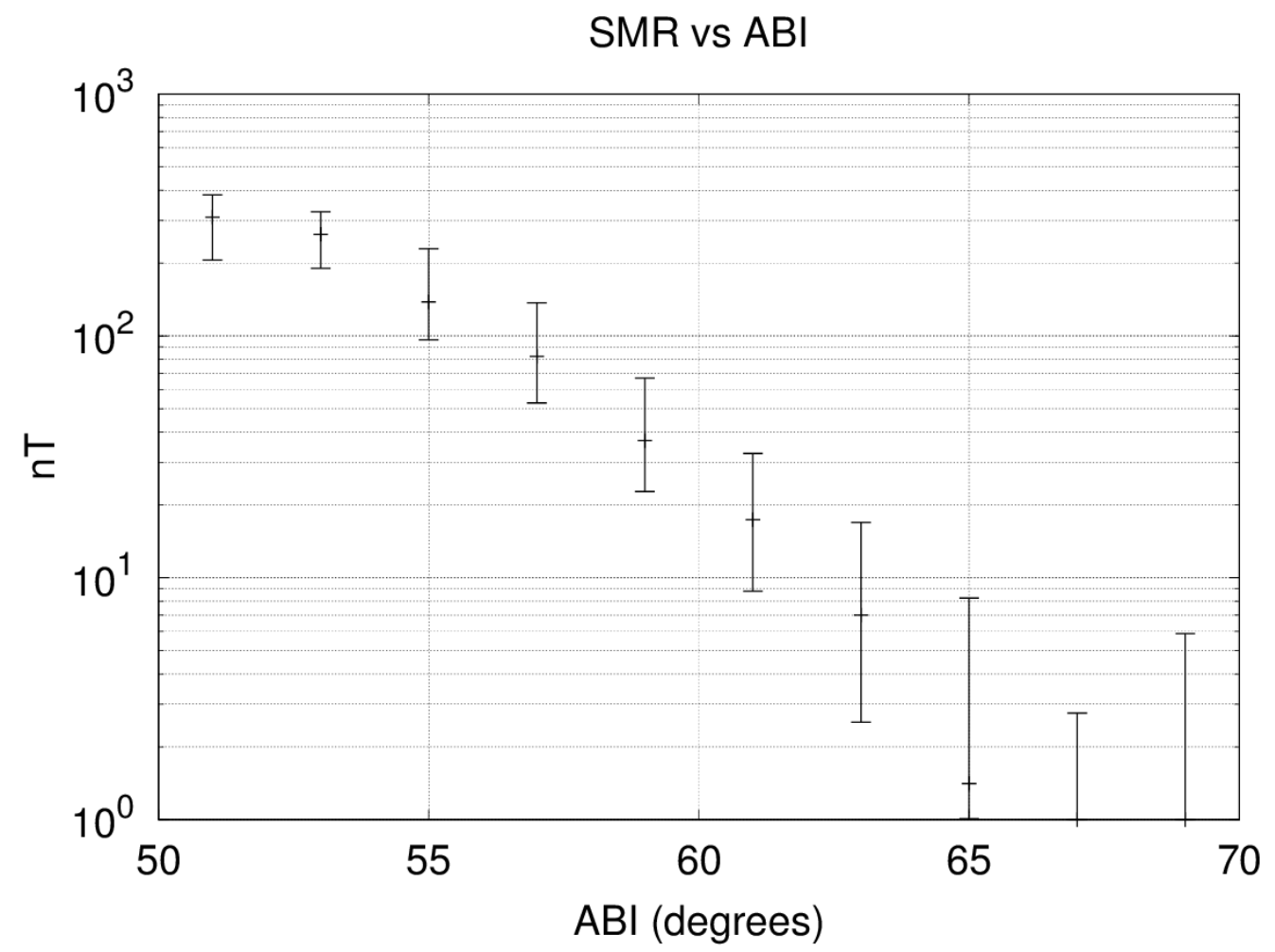

Figure 4.15: The magnitude of 10 minute average SMR index, for times when $S M R<0$, plus one is shown on a logarithmic scale versus ABI for years 2000-2009. For robustness, median values are plotted with mean deviation from the median, computed separately for values above and below the median, used for error bars. Note that SMR is less linear on this scaling than Dcx. 


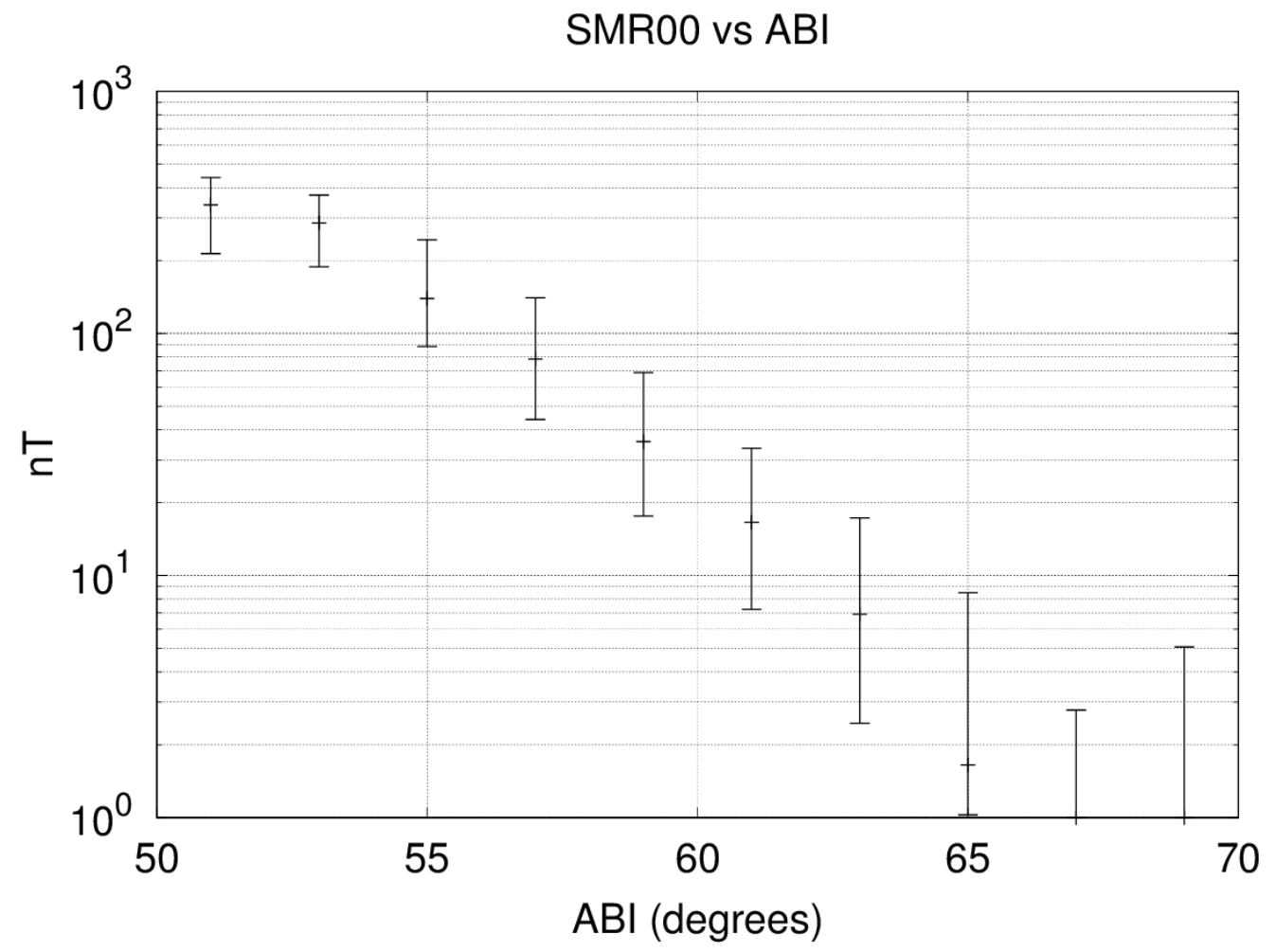

Figure 4.16: The magnitude of 10 minute average midnight sector SMR00 index, for times when $S M R 00<0$, plus one is shown on a logarithmic scale versus ABI for years 2000-2009. For robustness, median values are plotted with mean deviation from the median, computed separately for values above and below the median, used for error bars. 


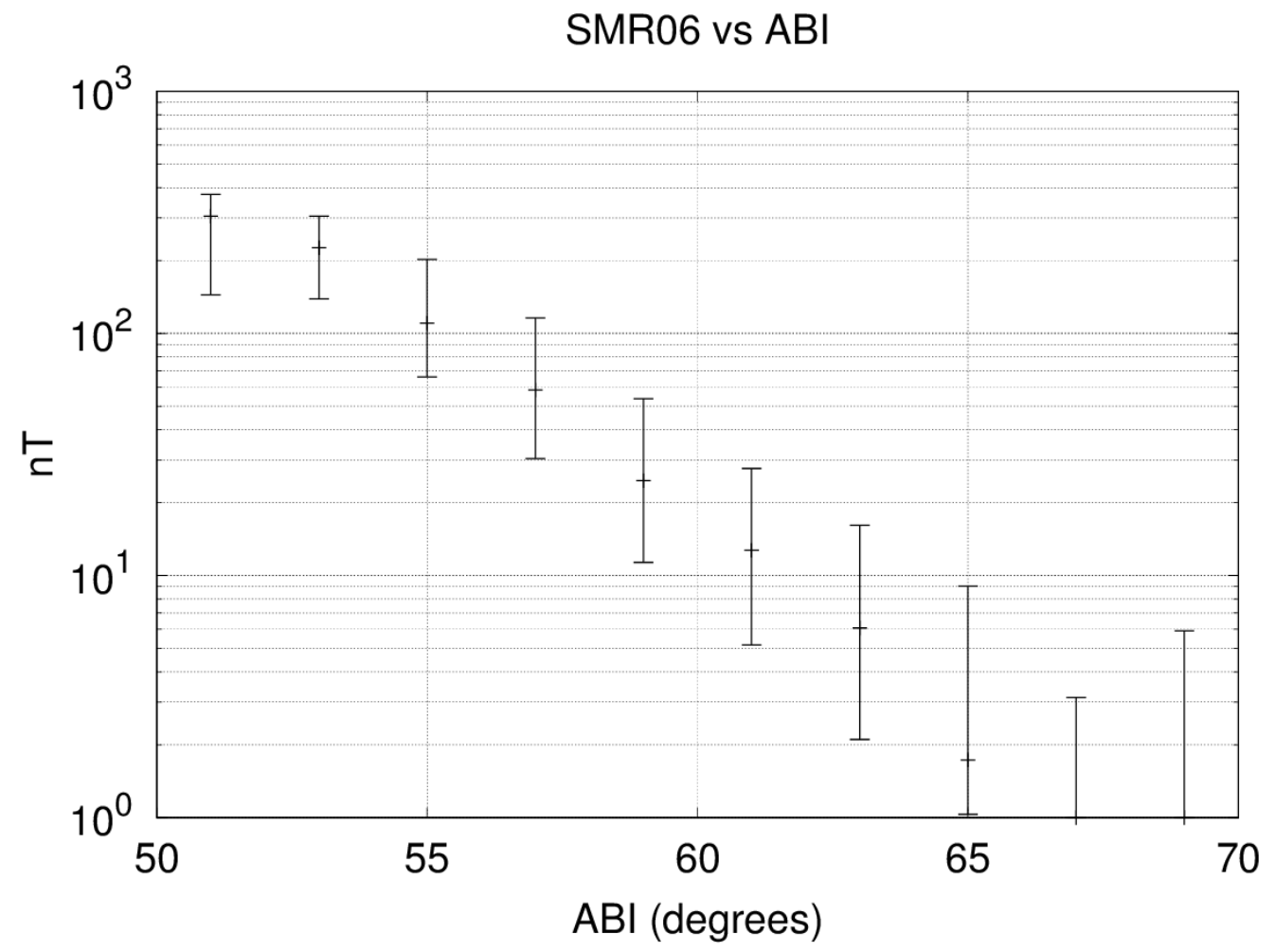

Figure 4.17: The magnitude of 10 minute average dawn sector SMR06 index, for times when $S M R 06<0$, plus one is shown on a logarithmic scale versus ABI for years 2000-2009. For robustness, median values are plotted with mean deviation from the median, computed separately for values above and below the median, used for error bars. 


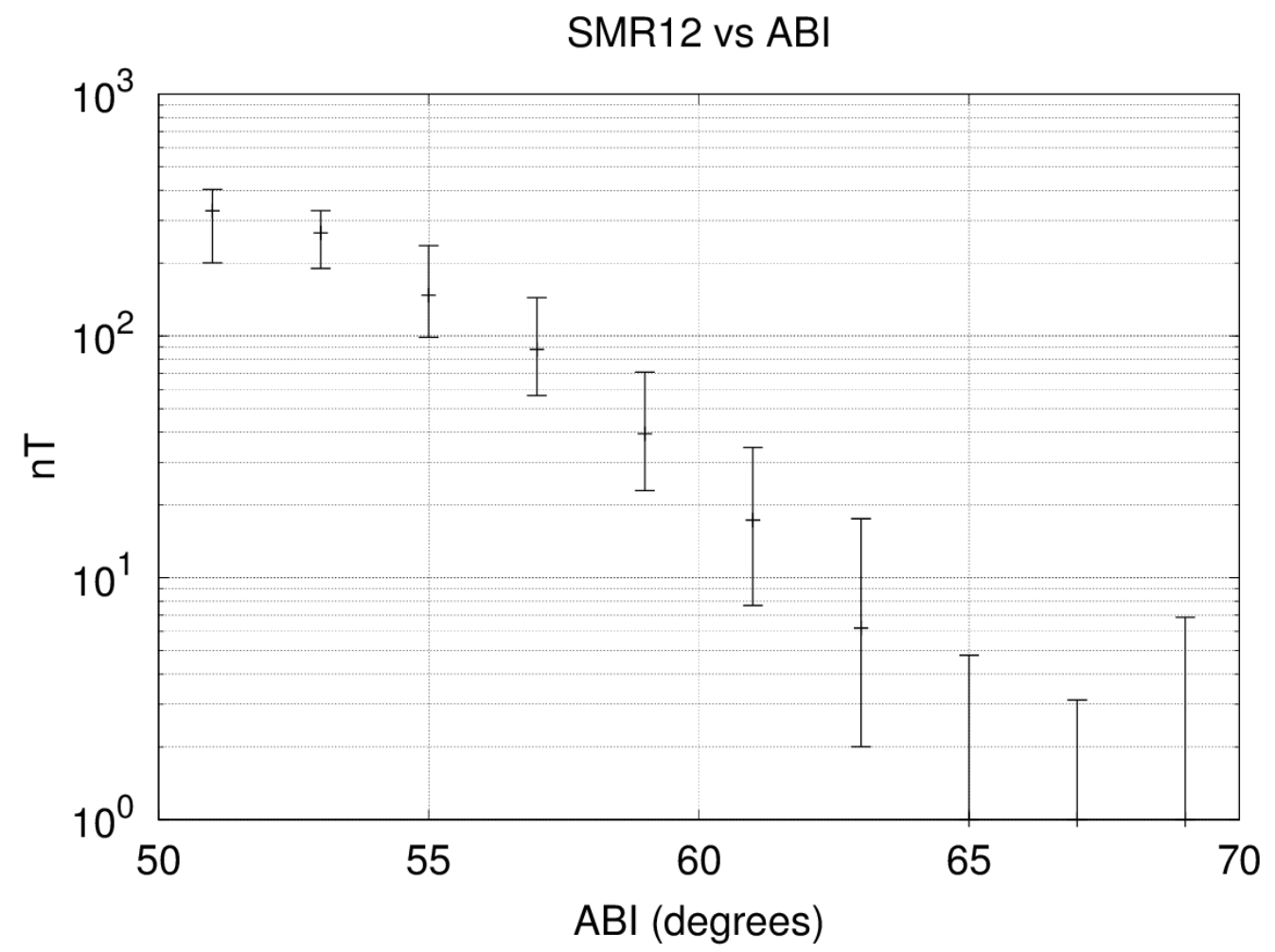

Figure 4.18: The magnitude of 10 minute average noon sector SMR12 index, for times when $S M R 06<0$, plus one is shown on a logarithmic scale versus ABI for years 2000-2009. For robustness, median values are plotted with mean deviation from the median, computed separately for values above and below the median, used for error bars. 


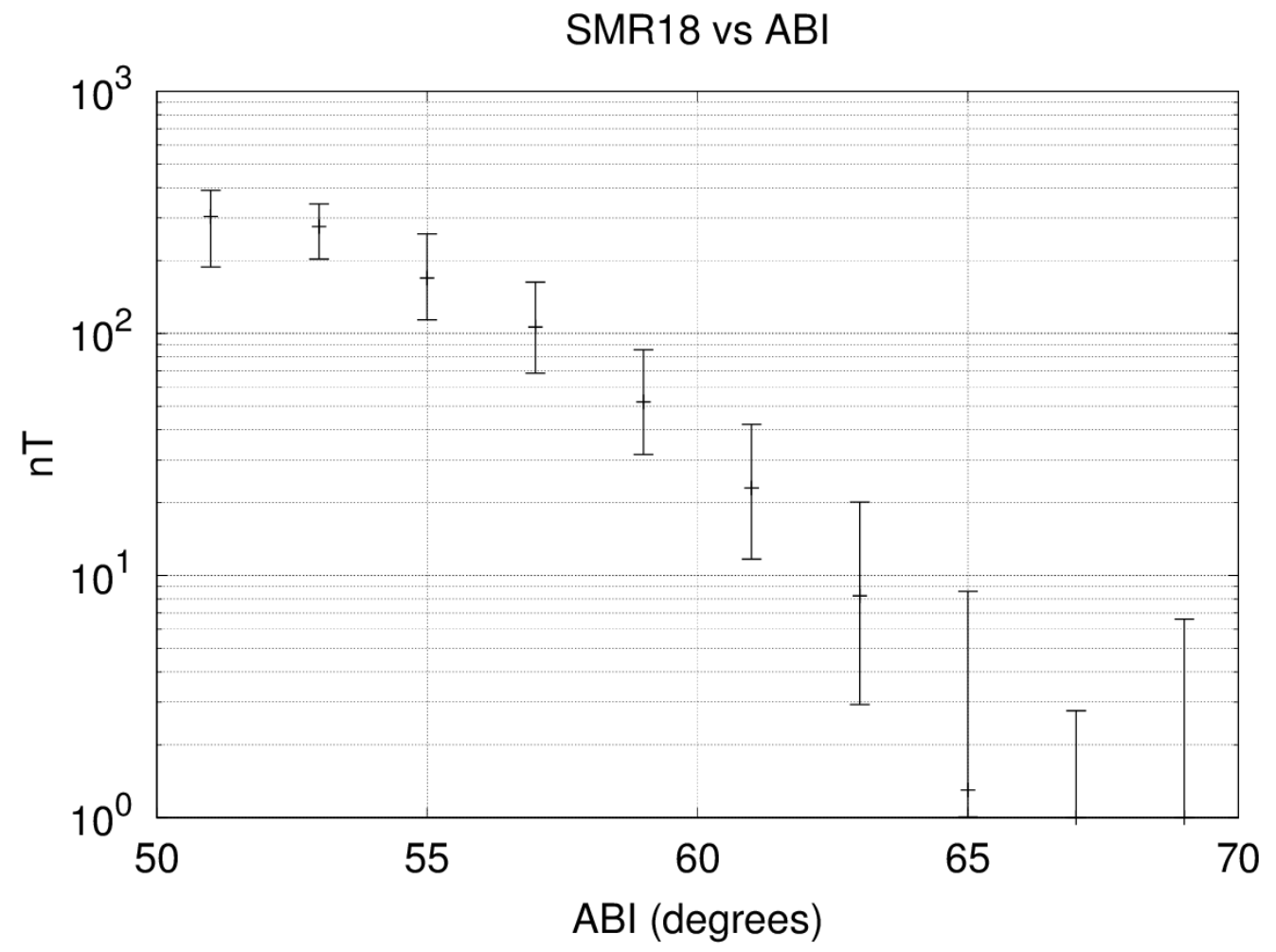

Figure 4.19: The magnitude of 10 minute average dusk sector SMR12 index, for times when $S M R 06<0$, plus one is shown on a logarithmic scale versus ABI for years 2000-2009. For robustness, median values are plotted with mean deviation from the median, computed separately for values above and below the median, used for error bars. 


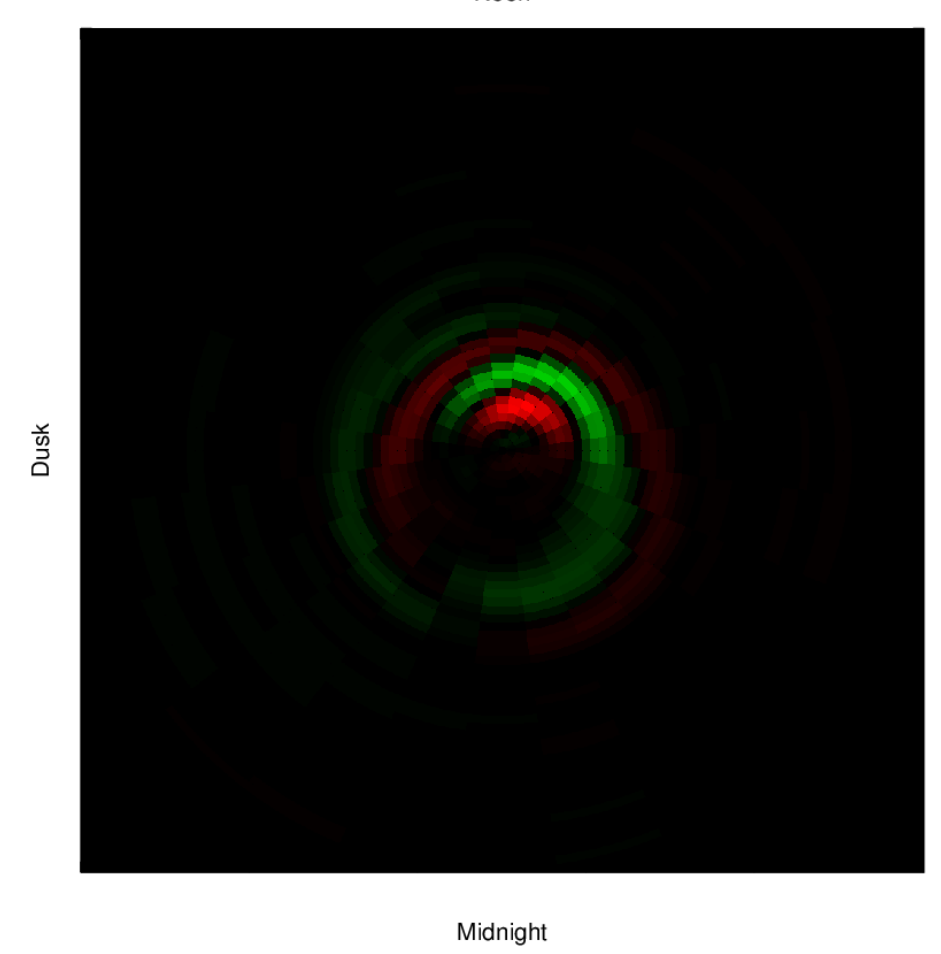

Figure 4.20: The field aligned currents for the northern hemisphere for a typical cluster when Dcx averages to $0 \mathrm{nT}$ is shown. Note the contracted, weak R1 and R2 currents, consistent with the tail dominated storm picture. Red represents upward current and green represents downward currents. 


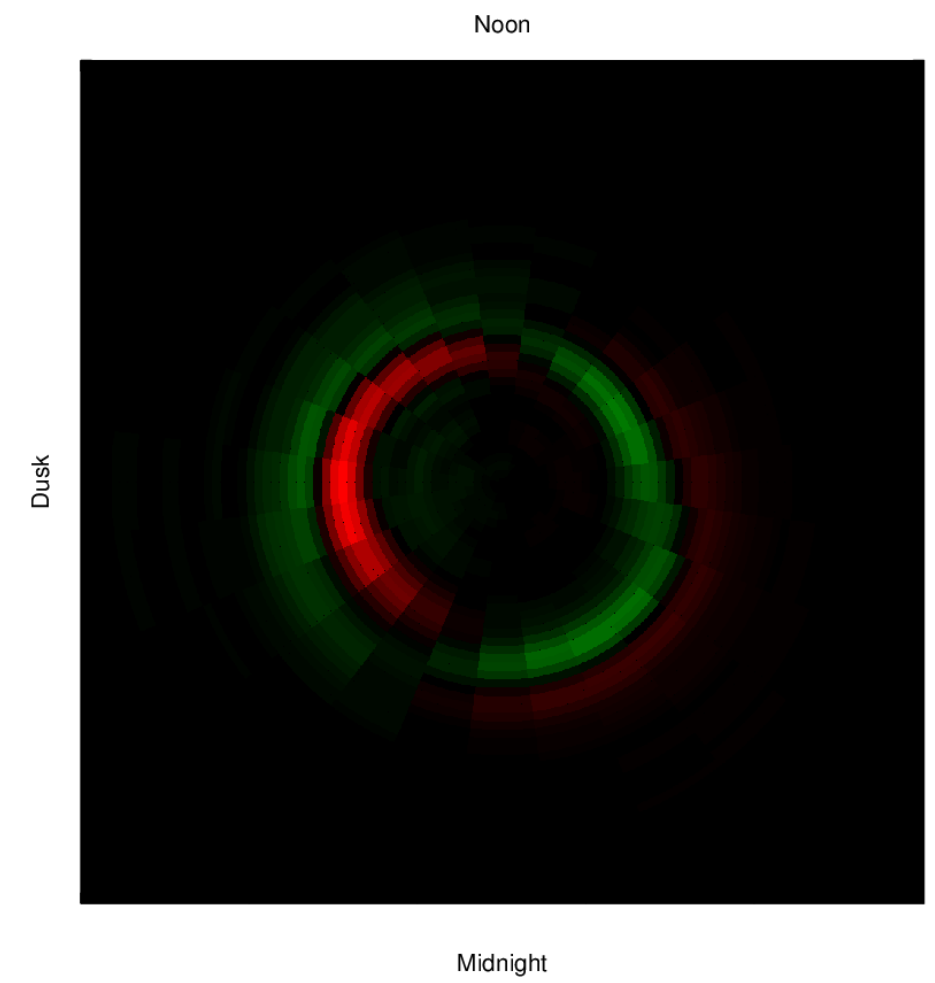

Figure 4.21: The field aligned currents for the northern hemisphere for a typical cluster when Dcx averages to $-25 \mathrm{nT}$ is shown. Note that R1 and R2 currents have expanded significantly from the $\mathrm{Dcx}=0 \mathrm{nT}$ case, consistent with the tail dominated storm picture. Red represents upward current and green represents downward currents. 


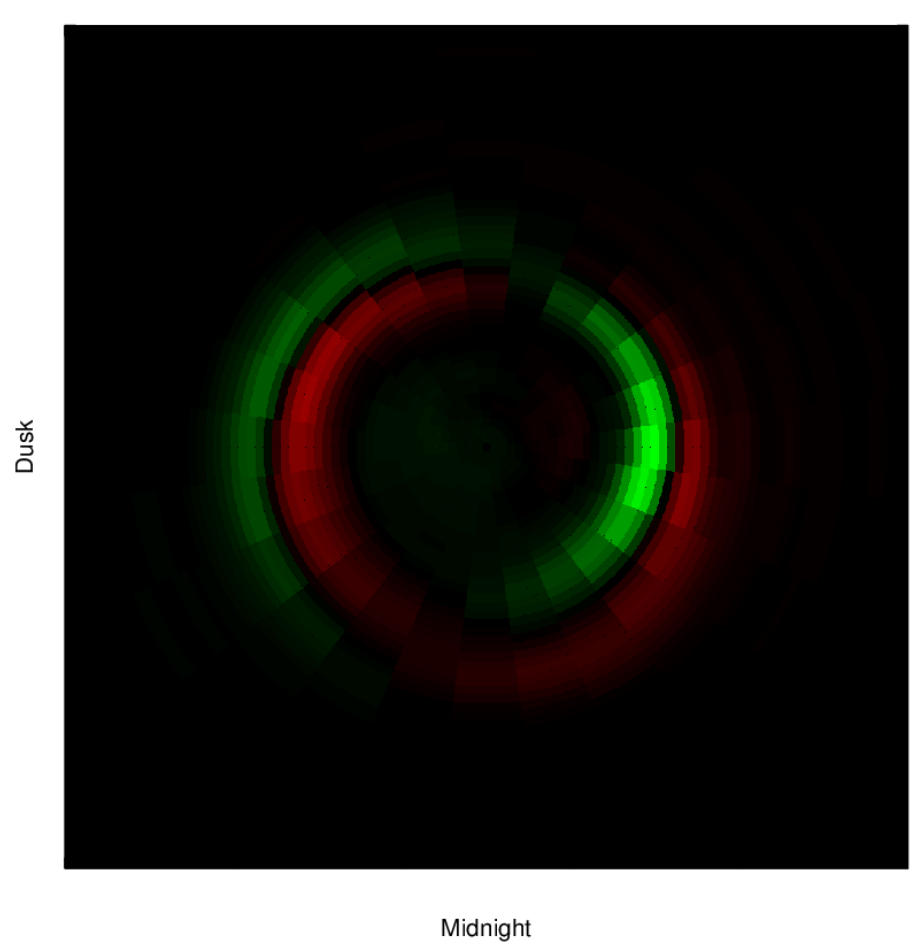

Figure 4.22: The field aligned currents for the northern hemisphere for a typical cluster when Dcx averages to $-49 \mathrm{nT}$ is shown. Note that R1 and R2 currents have expanded significantly from the Dcx $=-25 \mathrm{nT}$ case, consistent with the tail dominated storm picture. Red represents upward current and green represents downward currents. 


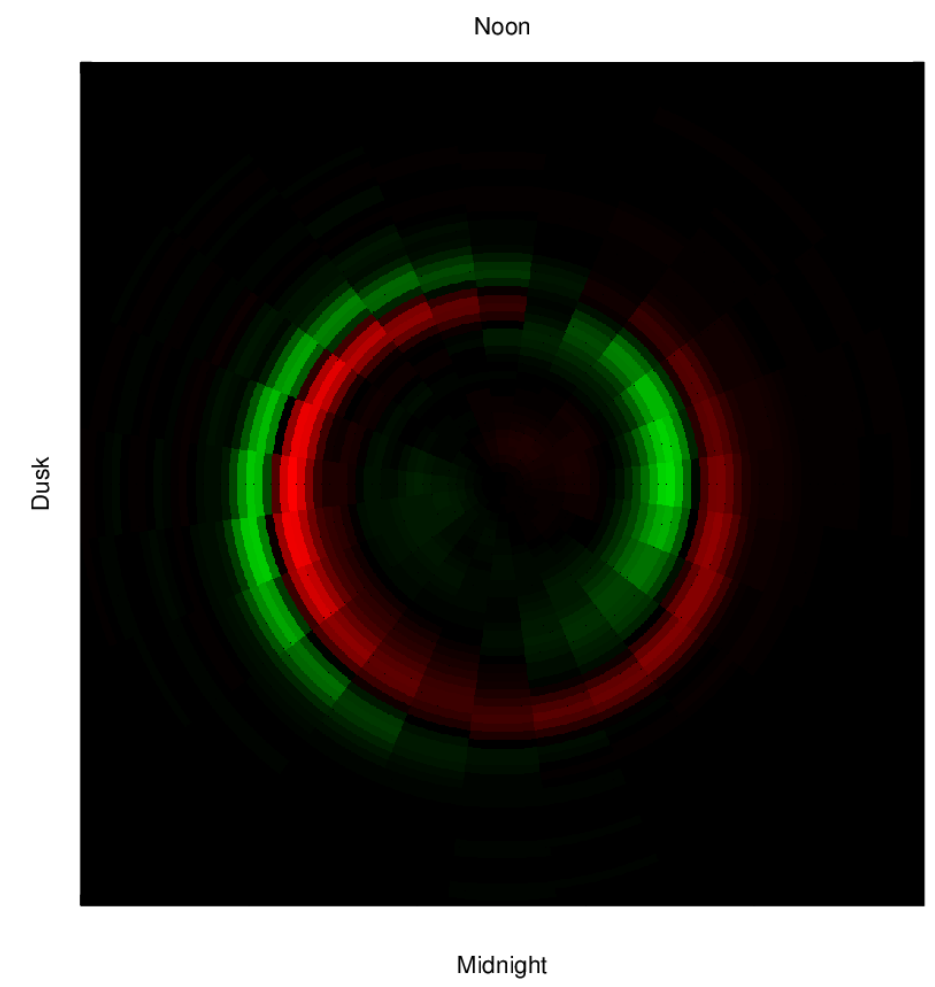

Figure 4.23: The field aligned currents for the northern hemisphere for a typical cluster when Dcx averages to $-75 \mathrm{nT}$ is shown. Note that $\mathrm{R} 1$ and $\mathrm{R} 2$ currents have expanded significantly from the Dcx $=-49 \mathrm{nT}$ case, consistent with the tail dominated storm picture. Red represents upward current and green represents downward currents. 


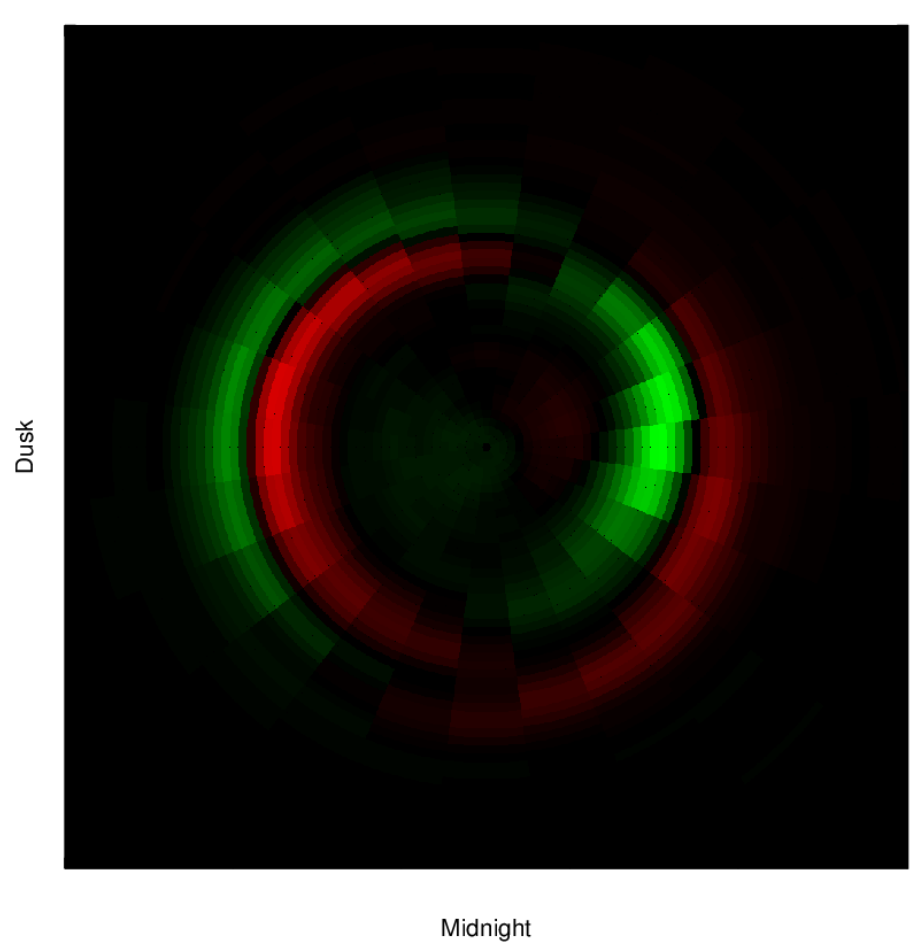

Figure 4.24: The field aligned currents for the northern hemisphere for a typical cluster when Dcx averages to $-104 \mathrm{nT}$ is shown. Note that R1 and R2 currents have expanded significantly from the Dcx $=-75 \mathrm{nT}$ case, consistent with the tail dominated storm picture. Red represents upward current and green represents downward currents. 


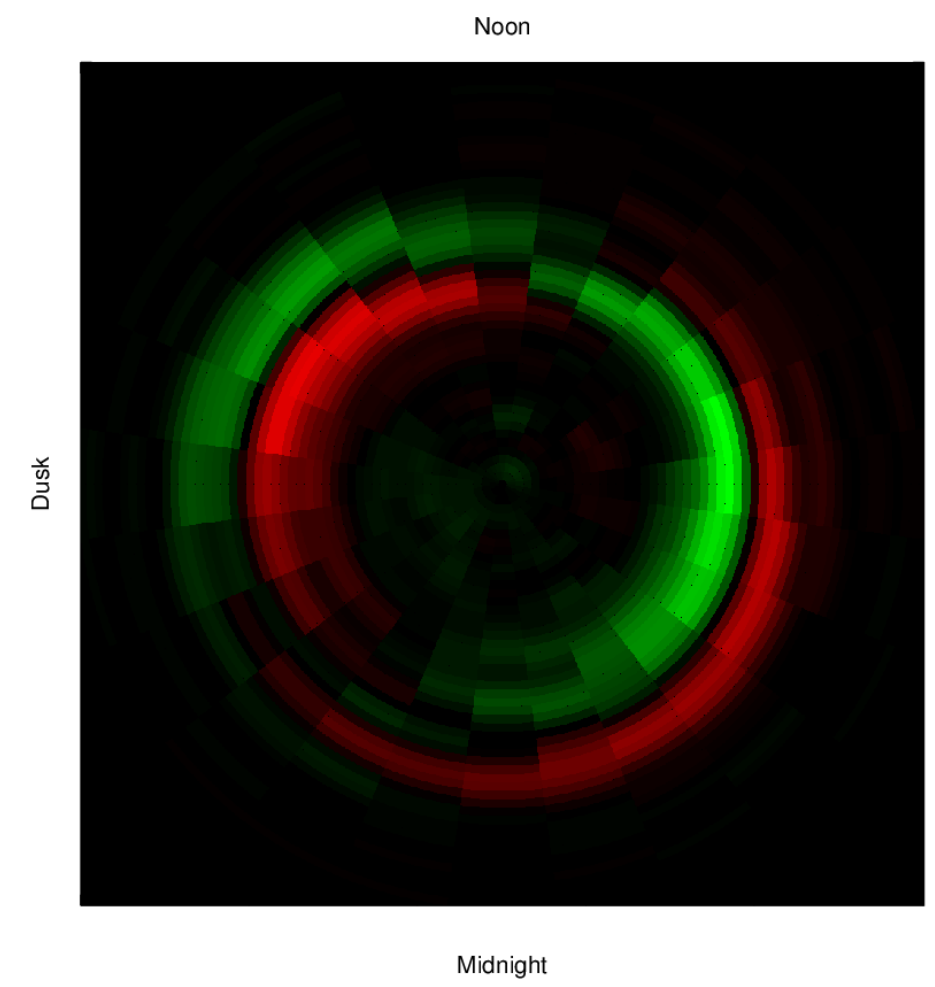

Figure 4.25: The field aligned currents for the northern hemisphere for a typical cluster when Dcx averages to $-159 \mathrm{nT}$ is shown. Note that R1 and R2 currents have expanded significantly from the Dcx $=-104 \mathrm{nT}$ case, consistent with the tail dominated storm picture. Red represents upward current and green represents downward currents. 

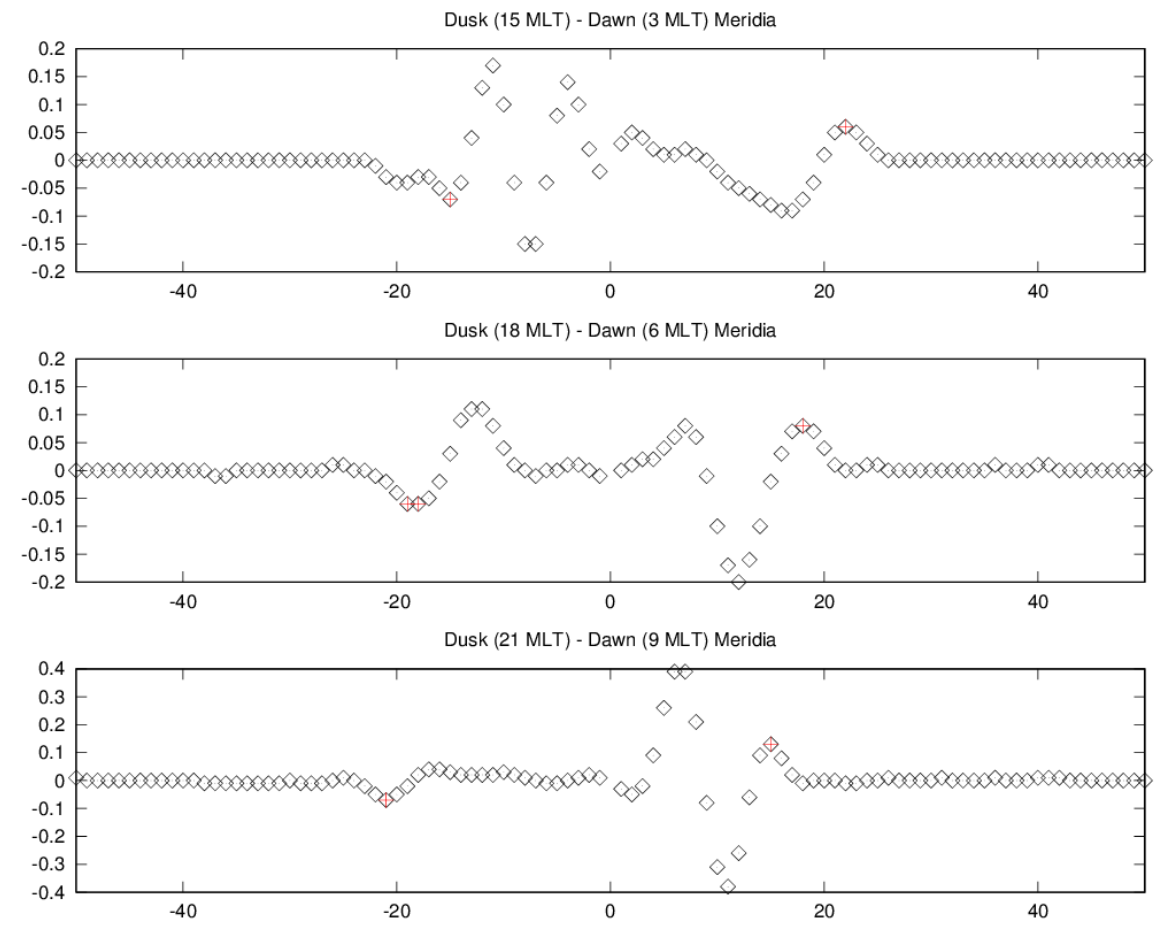

Figure 4.26: Plots of FAC intensity in $\mu \mathrm{A} / \mathrm{m}^{2}$ along the 3 meridia used for $\mathrm{R} 2$ FAC location identification corresponding to figure 4.20. The automatically identified R2 peaks are marked in red. 


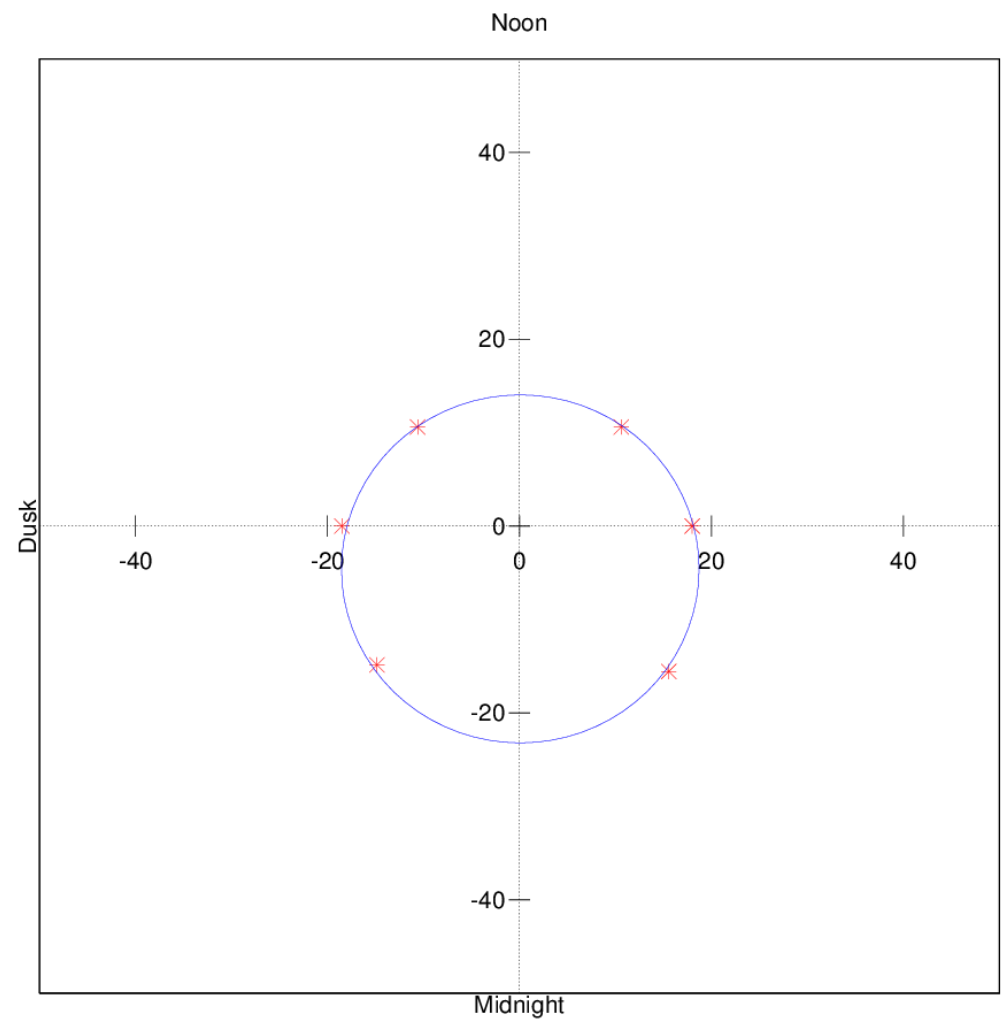

Figure 4.27: The 6 points identified in figure 4.26 as most likely R2 FAC peaks are marked. The circle shown was fit to these points and represents the most likely equatorward boundary of the auroral oval. 

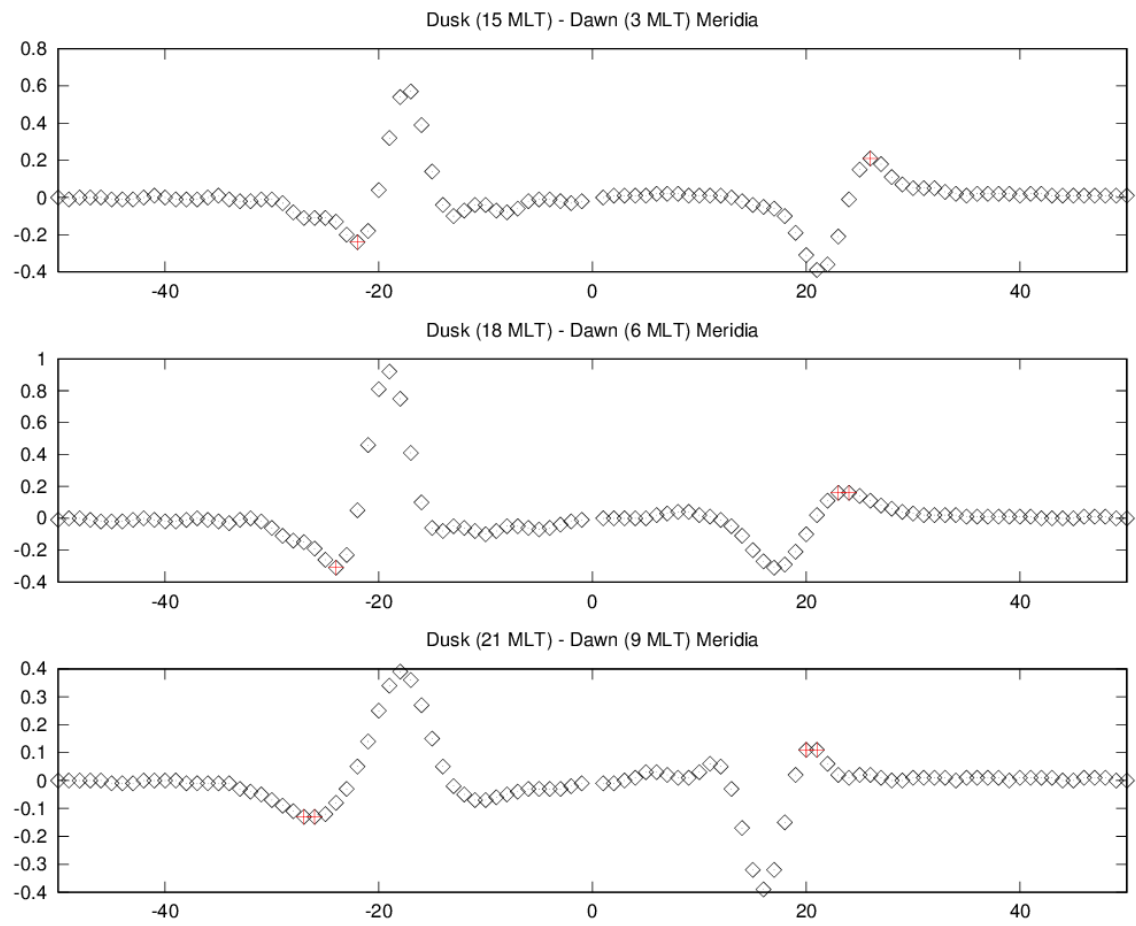

Figure 4.28: Plots of FAC intensity in $\mu \mathrm{A} / \mathrm{m}^{2}$ along the 3 meridia used for $\mathrm{R} 2$ FAC location identification corresponding to figure 4.21. The automatically identified R2 peaks are marked in red. 


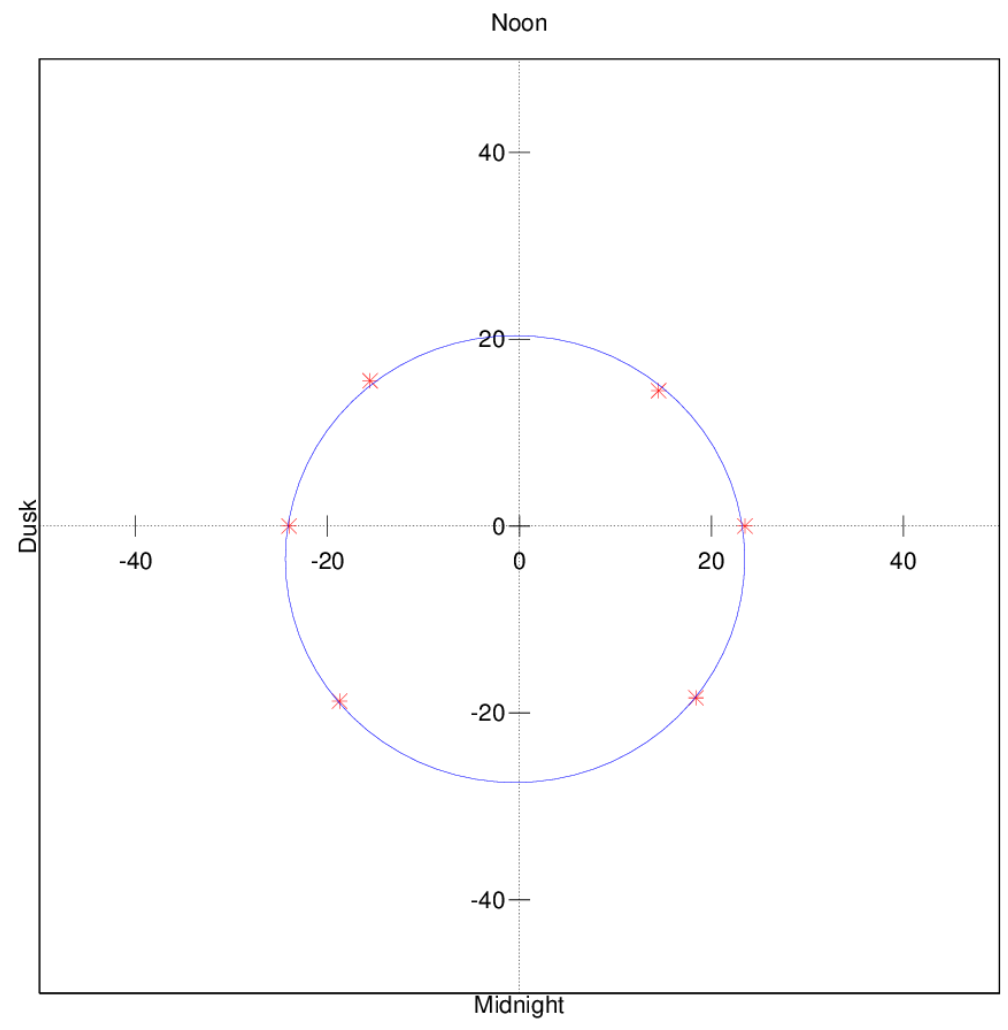

Figure 4.29: The 6 points identified in figure 4.28 as most likely R2 FAC peaks are marked. The circle shown was fit to these points and represents the most likely equatorward boundary of the auroral oval. 

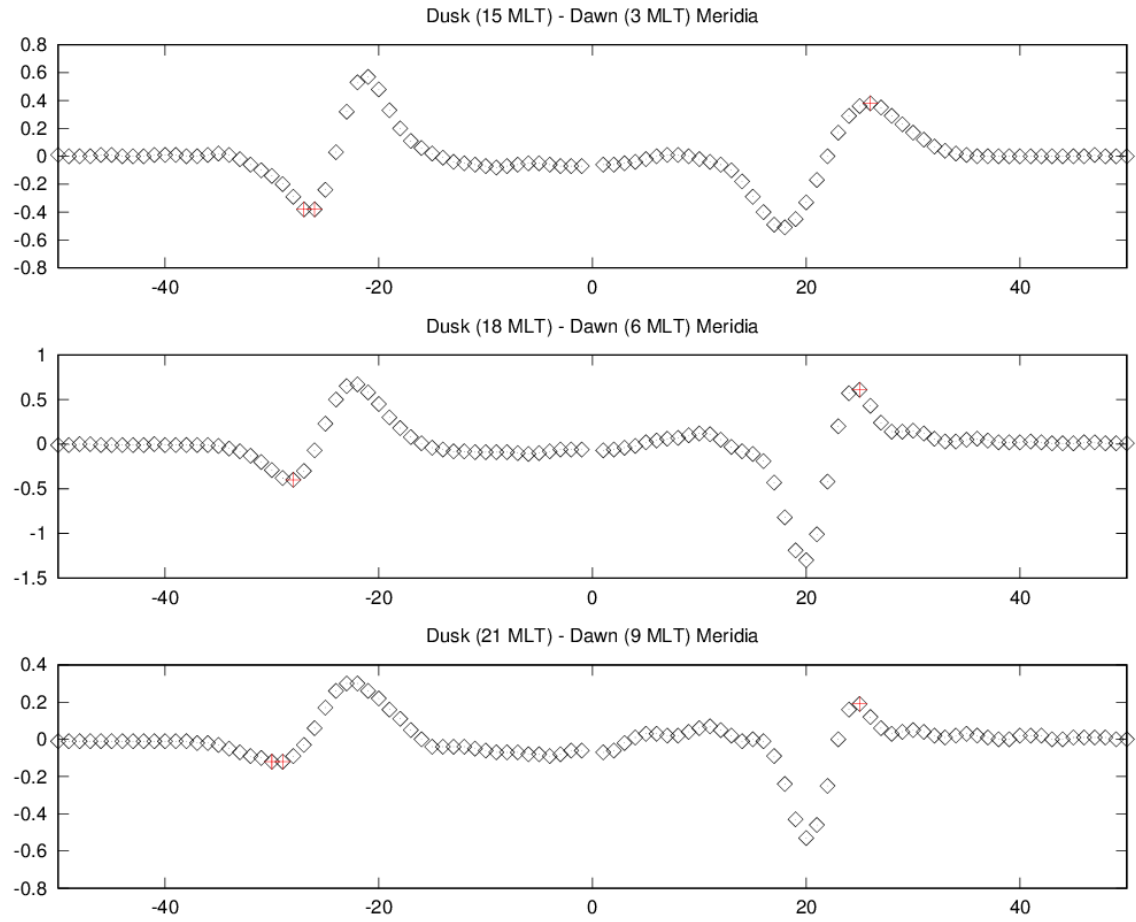

Figure 4.30: Plots of FAC intensity in $\mu \mathrm{A} / \mathrm{m}^{2}$ along the 3 meridia used for $\mathrm{R} 2$ FAC location identification corresponding to figure 4.22. The automatically identified R2 peaks are marked in red. 


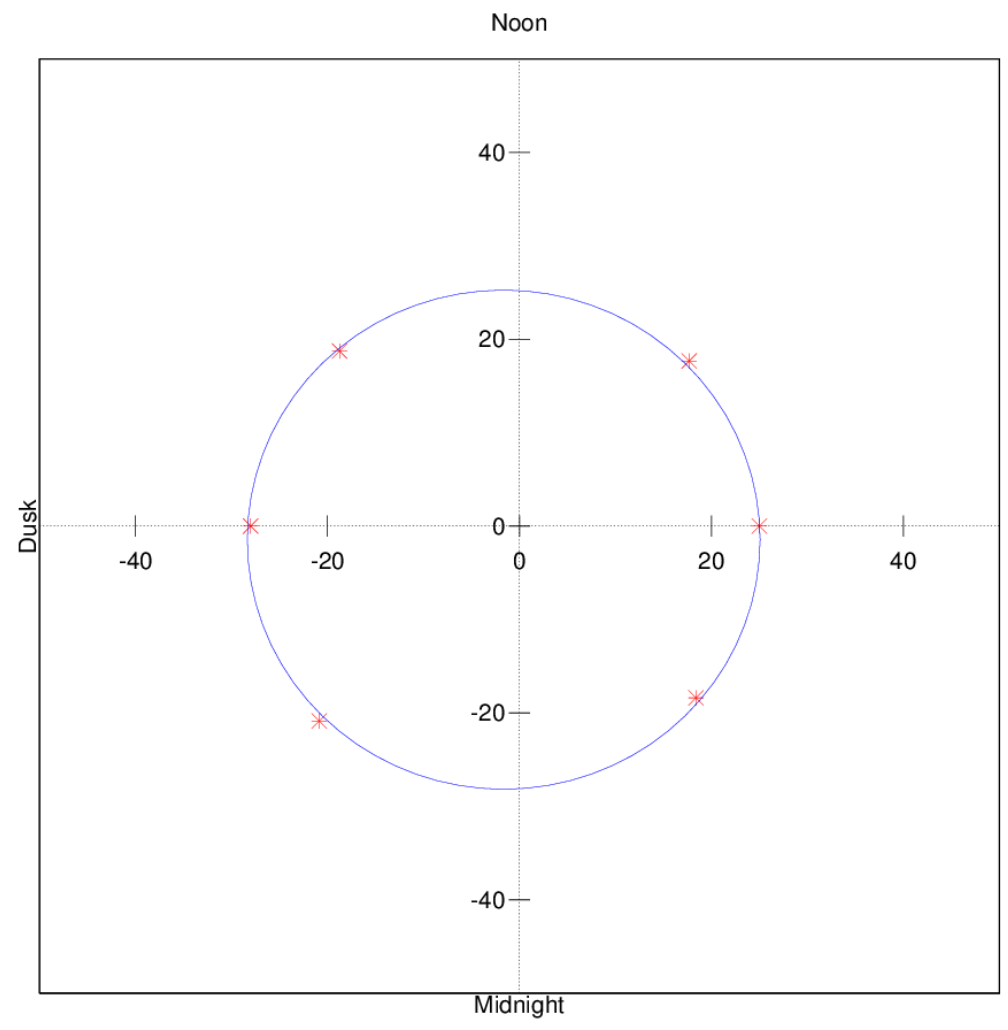

Figure 4.31: The 6 points identified in figure 4.30 as most likely R2 FAC peaks are marked. The circle shown was fit to these points and represents the most likely equatorward boundary of the auroral oval. 

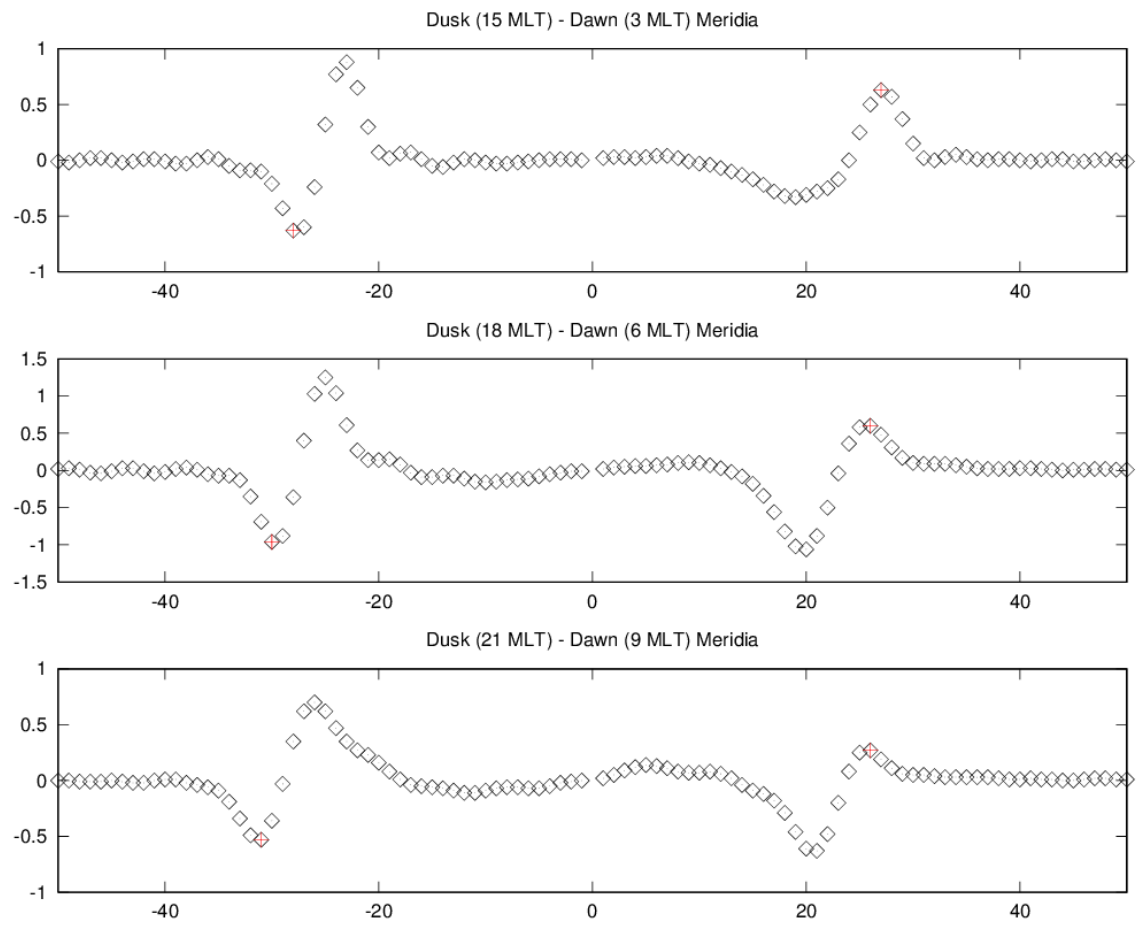

Figure 4.32: Plots of FAC intensity in $\mu \mathrm{A} / \mathrm{m}^{2}$ along the 3 meridia used for $\mathrm{R} 2$ FAC location identification corresponding to figure 4.23. The automatically identified R2 peaks are marked in red. 


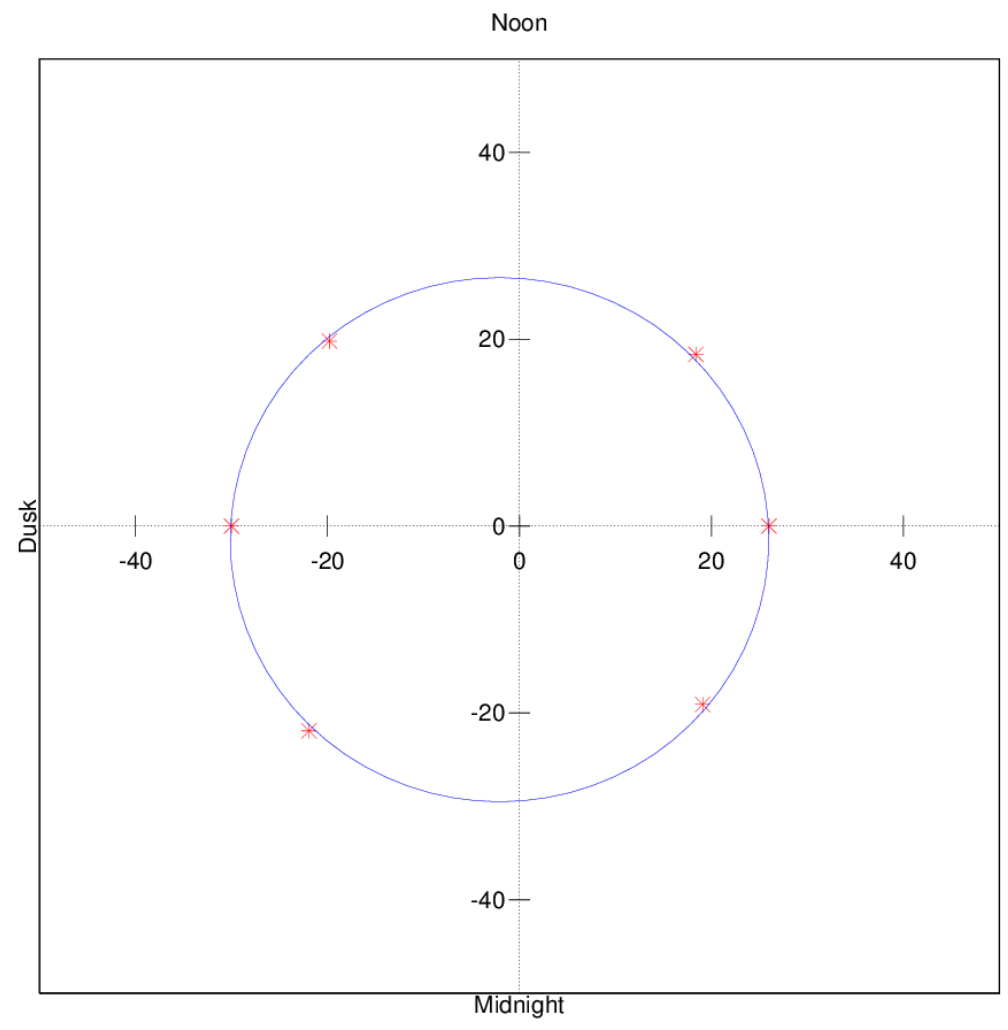

Figure 4.33: The 6 points identified in figure 4.32 as most likely R2 FAC peaks are marked. The circle shown was fit to these points and represents the most likely equatorward boundary of the auroral oval. 

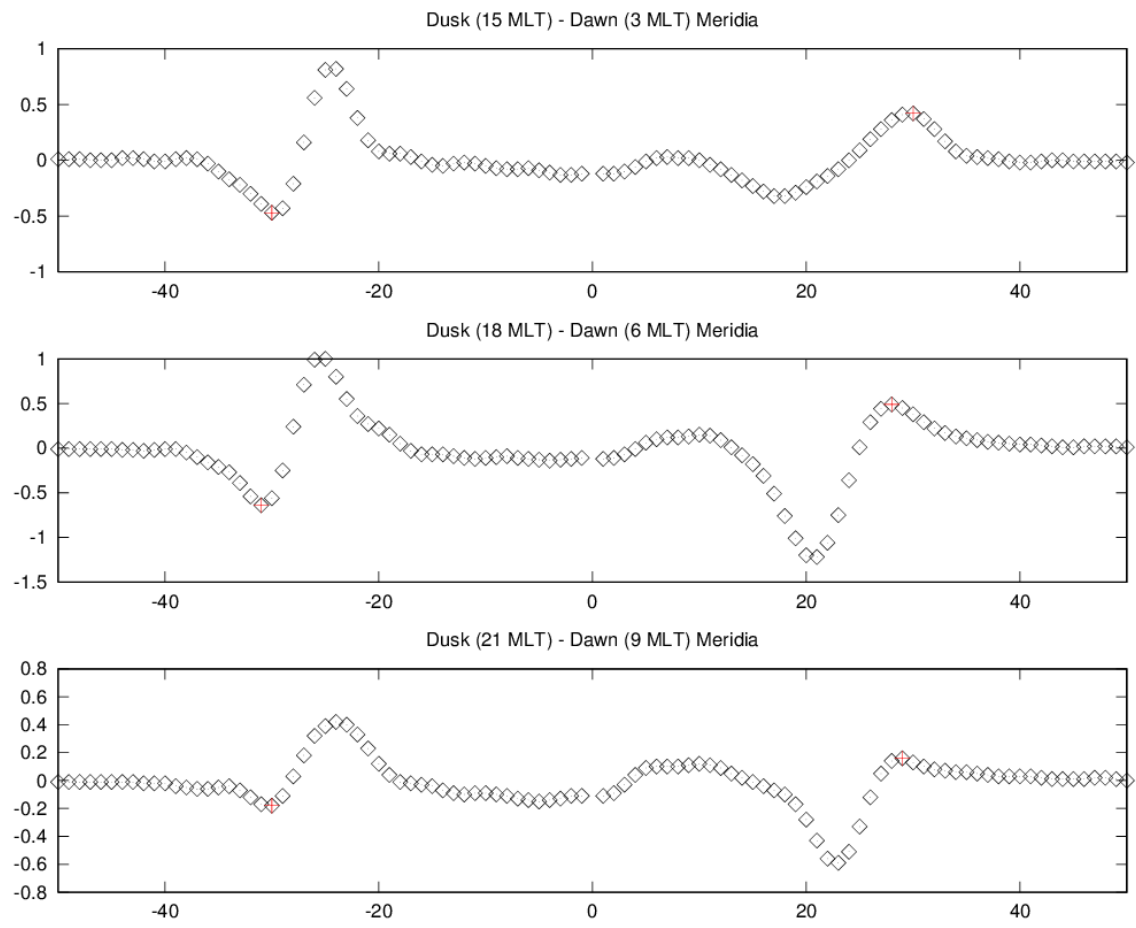

Figure 4.34: Plots of FAC intensity in $\mu \mathrm{A} / \mathrm{m}^{2}$ along the 3 meridia used for $\mathrm{R} 2$ FAC location identification corresponding to figure 4.24. The automatically identified R2 peaks are marked in red. 


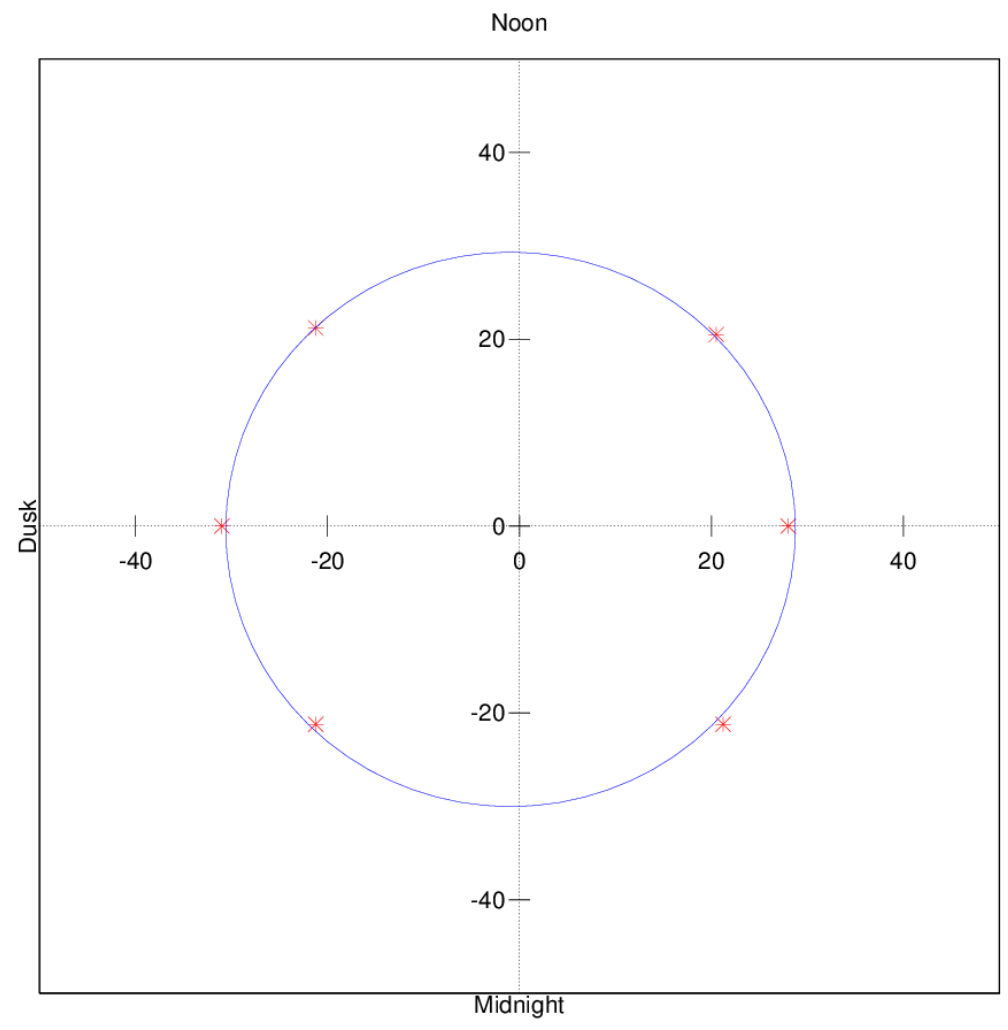

Figure 4.35: The 6 points identified in figure 4.34 as most likely R2 FAC peaks are marked. The circle shown was fit to these points and represents the most likely equatorward boundary of the auroral oval. 

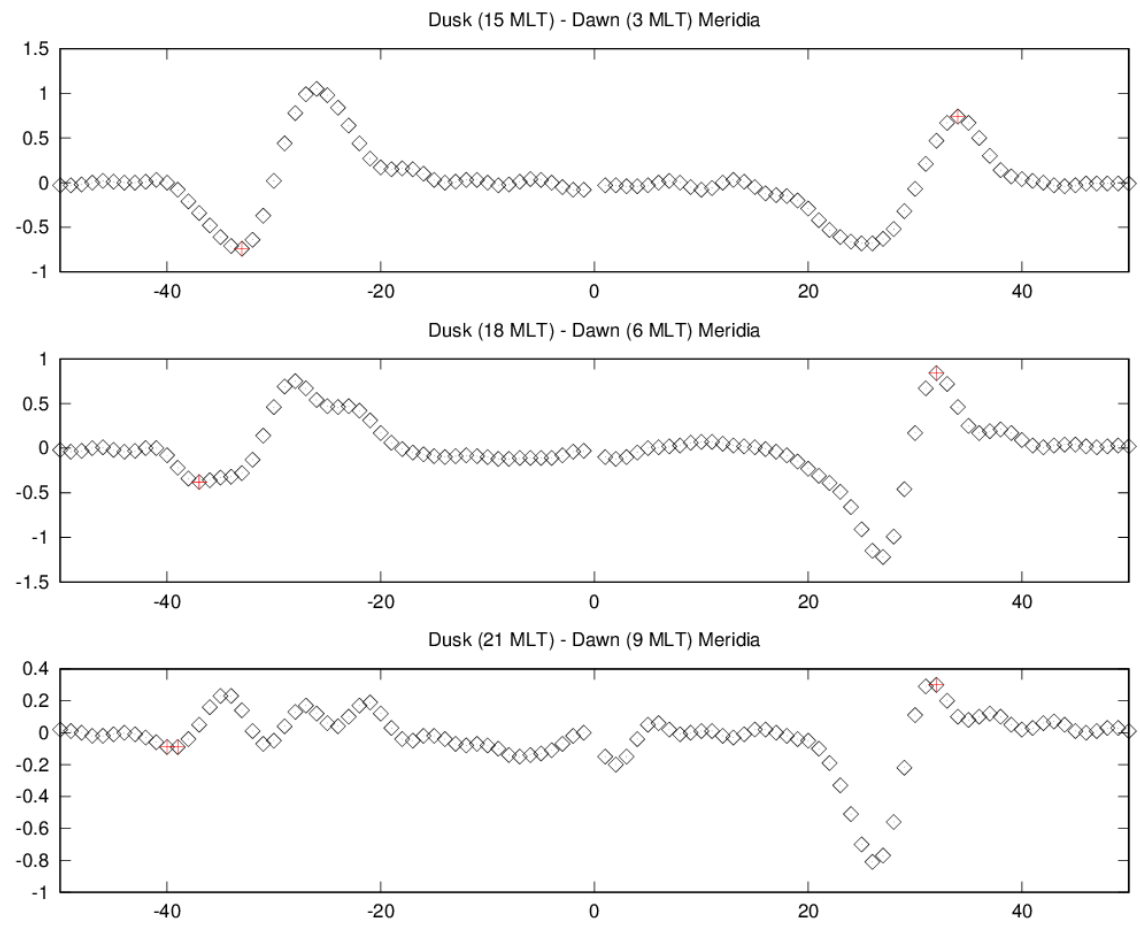

Figure 4.36: Plots of FAC intensity in $\mu \mathrm{A} / \mathrm{m}^{2}$ along the 3 meridia used for $\mathrm{R} 2$ FAC location identification corresponding to figure 4.25. The automatically identified R2 peaks are marked in red. 


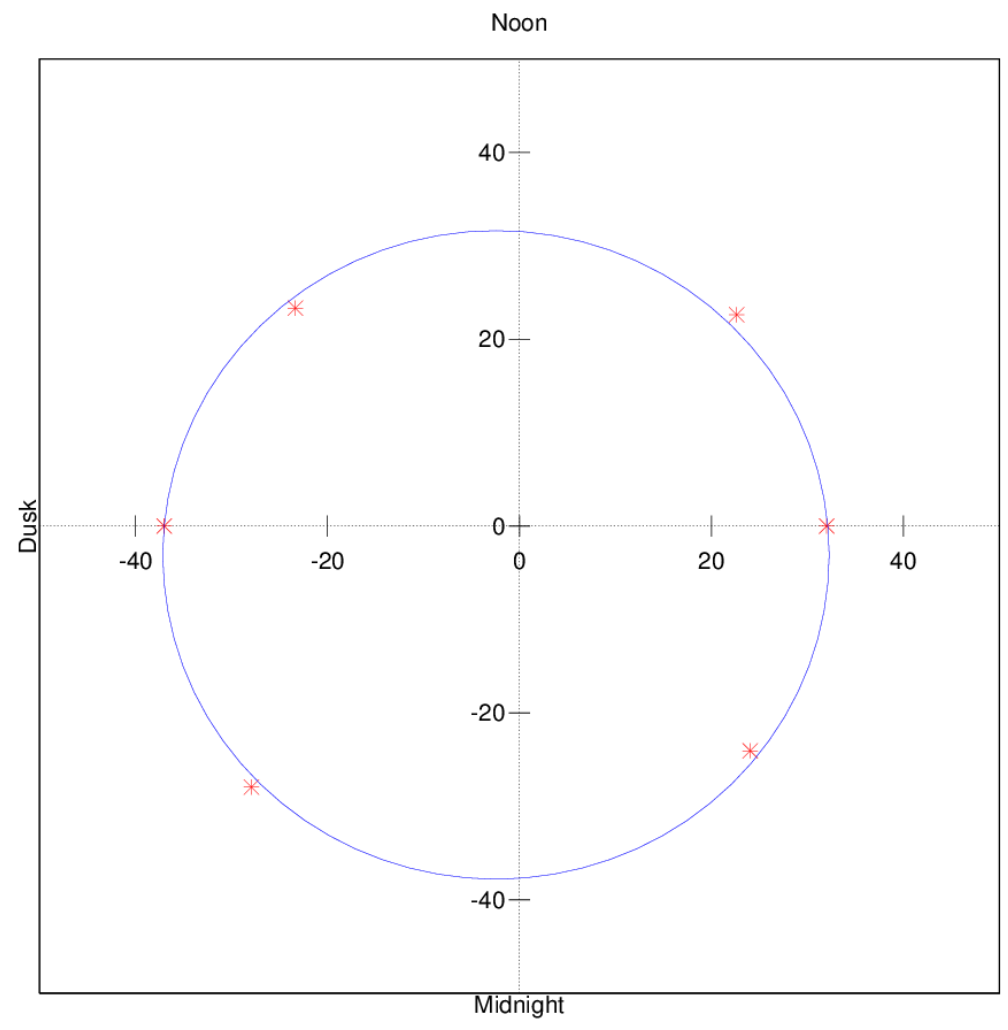

Figure 4.37: The 6 points identified in figure 4.36 as most likely R2 FAC peaks are marked. The circle shown was fit to these points and represents the most likely equatorward boundary of the auroral oval. 


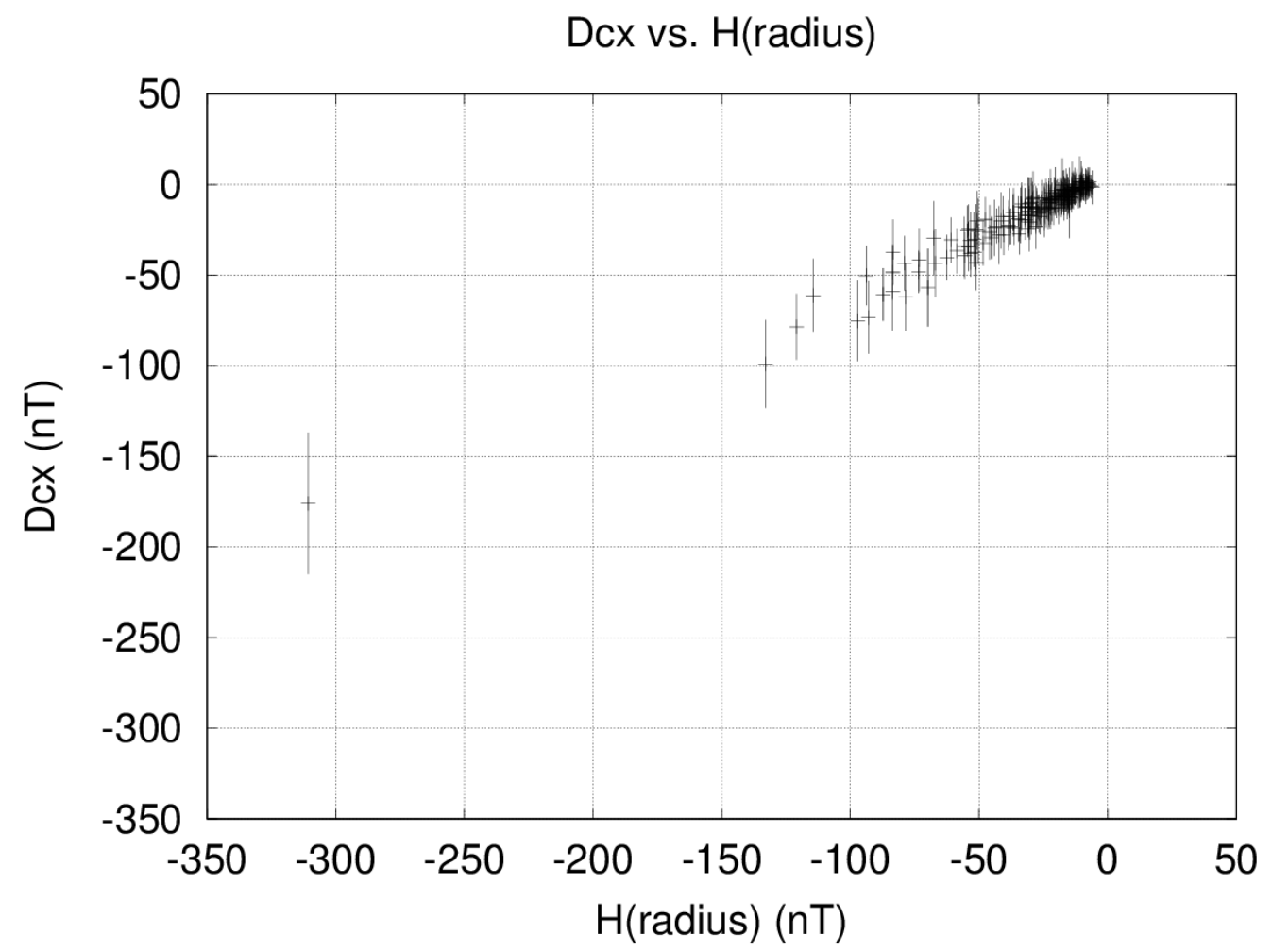

Figure 4.38: The median values of Dcx are plotted versus the theoretical cross tail current contribution $H(\theta)$, where $\theta$ is the radius of the auroral oval in degrees as determined from AMPERE data. Error bars represent the mean absolute deviation. 


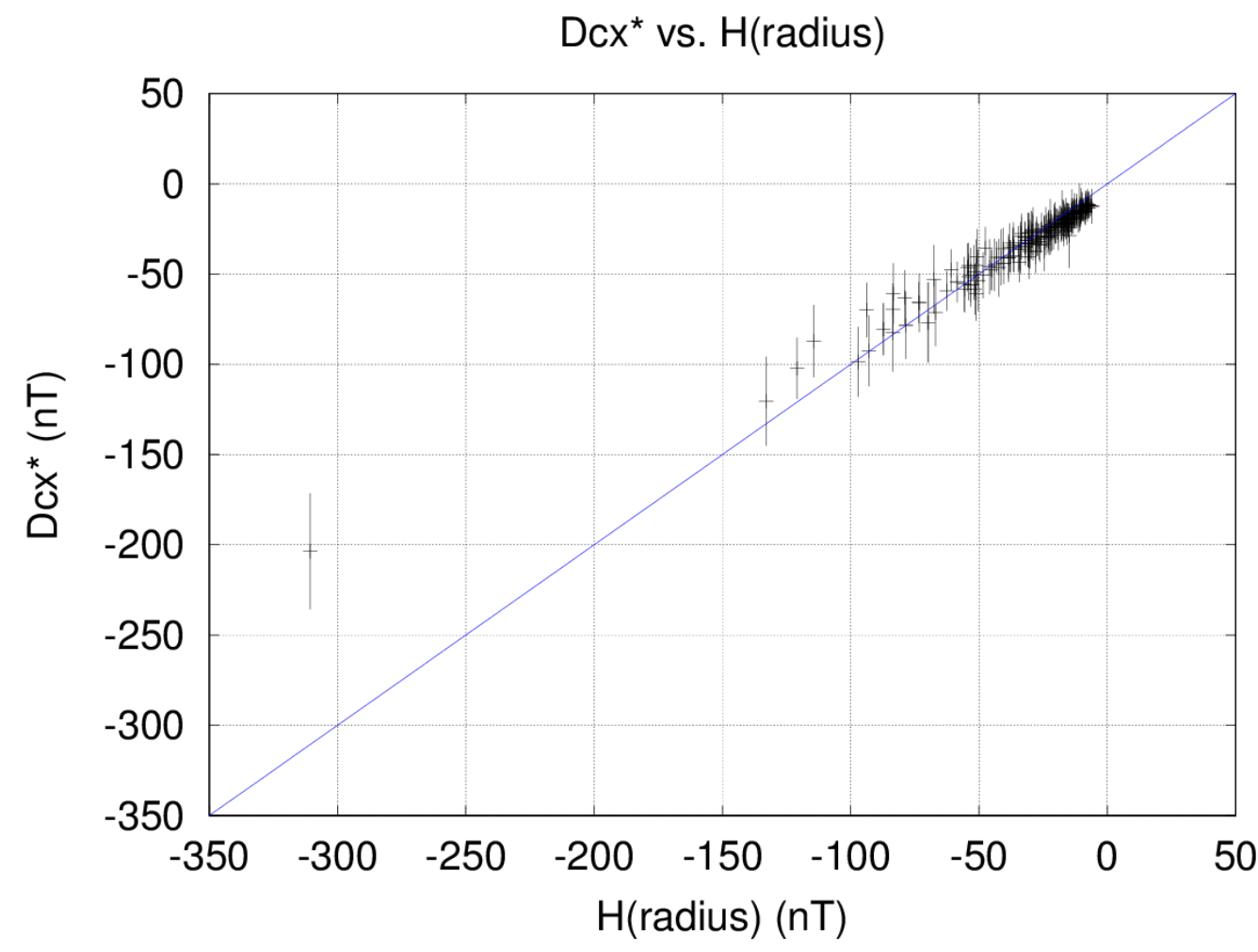

Figure 4.39: The median values of pressure corrected Dcx* are plotted versus the theoretical cross tail current contribution $H(\theta)$, where $\theta$ is the radius of the auroral oval in degrees as determined from AMPERE data. Error bars represent the mean absolute deviation. A line of slope 1 is added to guide the eye. 